Final Report

FHWA/IN/JTRP-2006/20

\title{
REDUCTION OF AUTOMOBILE AND AIRCRAFT COLLISIONS WITH WILDLIFE IN INDIANA
}

\author{
by \\ David J. Glista \\ Graduate Research Assistant \\ and \\ J. Andrew DeWoody \\ Associate Professor \\ and \\ Travis L. DeVault \\ Postdoctoral Associate \\ and \\ Olin E. Rhodes \\ Professor \\ Department of Forestry \& Natural Resources \\ Purdue University \\ Joint Transportation Research Program \\ Project No. C-36-67AAAA \\ File No. 9-10-78 \\ SPR-2925 \\ Conducted in Cooperation with the \\ Indiana Department of Transportation \\ and the U.S. Department of Transportation \\ Federal Highway Administration
}

The content of this report reflects the views of the authors who are responsible for the facts and the accuracy of the data presented herein. The contents do not necessarily reflect the official views or policies of the Indiana Department of Transportation or the Federal Highway Administration at the time of publication. This report does not constitute a standard, specification, or regulation.

Purdue University

West Lafayette, Indiana

December 2006 
TECHNICAL REPORT STANDARD TITLE PAGE

\begin{tabular}{|c|c|c|c|c|}
\hline $\begin{array}{l}\text { 1. Report No. } \\
\text { FHWA/IN/JTRP-2006/20 }\end{array}$ & \multicolumn{2}{|c|}{ 2. Government Accession No. } & \multicolumn{2}{|c|}{ 3. Recipient's Catalog No. } \\
\hline \multirow{2}{*}{\multicolumn{3}{|c|}{$\begin{array}{l}\text { 4. Title and Subtitle } \\
\text { Reduction of Automobile and Aircraft Collisions with Wildlife in Indiana }\end{array}$}} & \multicolumn{2}{|c|}{$\begin{array}{lc}\text { 5. } \quad \text { Report Date } \\
\text { December } 2006\end{array}$} \\
\hline & & & \multicolumn{2}{|c|}{ 6. Performing Organization Code } \\
\hline \multicolumn{3}{|c|}{$\begin{array}{l}\text { 7. Author(s) } \\
\text { David J. Glista, J. Andrew DeWoody, Travis L. DeVault, Olin E. Rhodes }\end{array}$} & \multicolumn{2}{|c|}{$\begin{array}{l}\text { 8. Performing Organization Report No. } \\
\text { FHWA/IN/JTRP-2006/20 }\end{array}$} \\
\hline \multirow[t]{2}{*}{$\begin{array}{l}\text { 9. Performing Organization Name and Address } \\
\text { Joint Transportation Research Program } \\
1284 \text { Civil Engineering Building } \\
\text { Purdue University } \\
\text { West Lafayette, IN 47907-1284 }\end{array}$} & & & \multicolumn{2}{|l|}{ 10. Work Unit No. } \\
\hline & & & \multicolumn{2}{|c|}{$\begin{array}{l}\text { 11. Contract or Grant No. } \\
\text { SPR-2925 }\end{array}$} \\
\hline \multirow{2}{*}{\multicolumn{3}{|c|}{$\begin{array}{l}\text { 12. Sponsoring Agency Name and Address } \\
\text { Indiana Department of Transportation } \\
100 \text { North Senate Avenue } \\
\text { Indianapolis, IN } 46204\end{array}$}} & \multicolumn{2}{|c|}{$\begin{array}{l}\text { 13. Type of Report and Period Covered } \\
\text { Final Report }\end{array}$} \\
\hline & & & \multicolumn{2}{|c|}{ 14. Sponsoring Agency Code } \\
\hline \multicolumn{5}{|c|}{$\begin{array}{l}\text { 15. Supplementary Notes } \\
\text { Prepared in cooperation with the Indiana Department of Transportation and Federal Highway Administration. }\end{array}$} \\
\hline \multicolumn{5}{|c|}{ 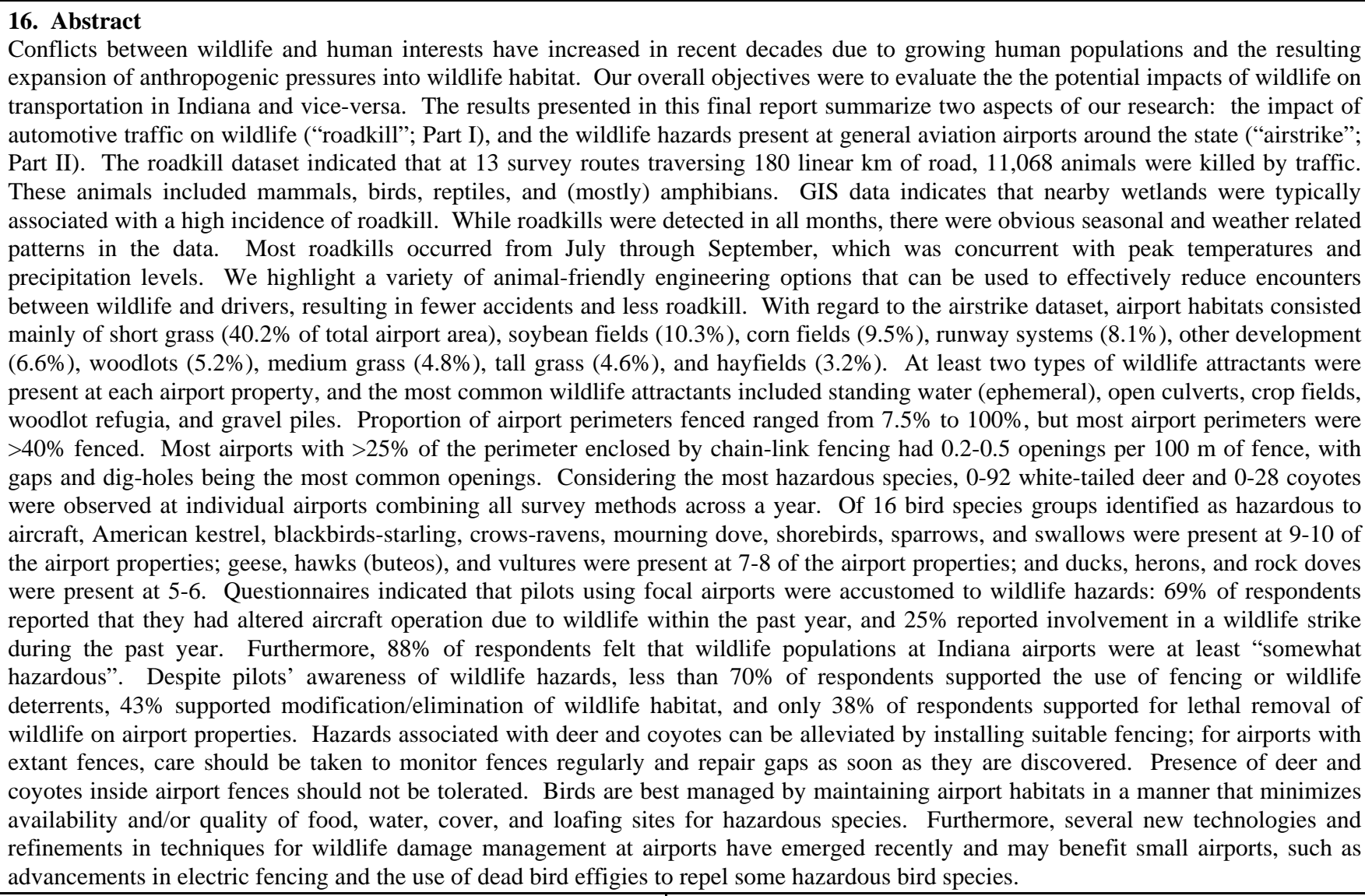 } \\
\hline $\begin{array}{l}\text { 17. Key Words } \\
\text { wildlife, transportation, airstrike } \\
\text { human/wildlife conflict }\end{array}$ & adkill, vertebrate, & $\begin{array}{l}\text { 18. Distribution } \\
\text { No restrictior } \\
\text { National Tec }\end{array}$ & $\begin{array}{l}\text { document is av } \\
\text { formation Servi }\end{array}$ & $\begin{array}{l}\text { the public through the } \\
\text { gfield, VA } 22161\end{array}$ \\
\hline 19. Security Classif. (of this report) & 20. Security Classif. (of & is page) & 21. No. of Pages & 22. Price \\
\hline Unclassified & Unclassifi & & 201 & \\
\hline
\end{tabular}

Form DOT F 1700.7 (8-69) 
Final Report

FHWA/JTRP-2006/25

\title{
REDUCTION OF AUTOMOBILE AND AIRCRAFT COLLISIONS WITH WILDLIFE IN INDIANA
}

\author{
by \\ David J. Glista \\ Graduate Research Assistant \\ and \\ J. Andrew DeWoody \\ Associate Professor \\ and \\ Travis L. DeVault \\ Postdoctoral Associate \\ and \\ Olin E. Rhodes \\ Professor \\ Department of Forestry \& Natural Resources \\ Purdue University \\ Joint Transportation Research Program \\ File No. 9-10-78 \\ SPR-2925 \\ Conducted in Cooperation with the \\ Indiana Department of Transportation \\ and the U.S. Department of Transportation \\ Federal Highway Administration
}

The content of this report reflects the views of the authors who are responsible for the facts and the accuracy of the data presented herein. The contents do not necessarily reflect the official views or policies of the Indiana Department of Transportation or the Federal Highway Administration at the time of publication. This report does not constitute a standard, specificiation, or regulation.

Purdue University

December 2006 


\section{ACKNOWLEDGEMENTS}

We thank the Joint Transportation Research Program (Indiana Department of

Transportation and Purdue University), the Aviation Association of Indiana, South Bend Regional Airport, Warsaw Municipal Airport, DeKalb County Airport, and the Department of Forestry and Natural Resources at Purdue University for funding these studies. We thank all of the airport managers who allowed us access to airport properties and provided logistical support. Bart Giesler, Jim Keefer, Andy Nahrwold, and Rod Williams provided advice on various aspects of the study. We especially thank Jake Kubel and Dustin McBride for their assistance in the field. 


\section{ABSTRACT}

Conflicts between wildlife and human interests have increased in recent decades due to growing human populations and the resulting expansion of anthropogenic pressures into wildlife habitat. Our overall objectives were to evaluate the the potential impacts of wildlife on transportation in Indiana and vice-versa. The results presented in this final report summarize two aspects of our research: the impact of automotive traffic on wildlife ("roadkill"; Part I), and the wildlife hazards present at general aviation airports around the state ("airstrike"; Part II). The roadkill dataset indicated that at 13 survey routes traversing 180 linear $\mathrm{km}$ of road, 11,068 animals were killed by traffic. These animals included mammals, birds, reptiles, and (mostly) amphibians. GIS data indicates that nearby wetlands were typically associated with a high incidence of roadkill. While roadkills were detected in all months, there were obvious seasonal and weather related patterns in the data. Most roadkills occurred from July through September, which was concurrent with peak temperatures and precipitation levels. We highlight a variety of animal-friendly engineering options that can be used to effectively reduce encounters between wildlife and drivers, resulting in fewer accidents and less roadkill. With regard to the airstrike dataset, airport habitats consisted mainly of short grass (40.2\% of total airport area), soybean fields $(10.3 \%)$, corn fields $(9.5 \%)$, runway systems $(8.1 \%)$, other development $(6.6 \%)$, woodlots $(5.2 \%)$, medium grass $(4.8 \%)$, tall grass $(4.6 \%)$, and hayfields (3.2\%). At least two types of wildlife attractants were present at each airport property, and the most common wildlife attractants included standing water (ephemeral), open culverts, crop fields, woodlot refugia, and gravel piles. Proportion of airport perimeters fenced ranged from $7.5 \%$ to $100 \%$, but most airport perimeters were $>40 \%$ fenced. Most airports with $>25 \%$ of the perimeter enclosed by chain-link fencing had 0.2-0.5 openings per $100 \mathrm{~m}$ of fence, with gaps and dig-holes being the most common openings. Considering the most hazardous species, 0-92 white-tailed deer and 0-28 coyotes were observed at individual airports combining all survey methods across a year. Of 16 bird species groups identified as hazardous to aircraft, American kestrel, blackbirds-starling, crows-ravens, mourning dove, shorebirds, sparrows, and swallows were present at 9-10 of the airport properties; geese, hawks (buteos), and vultures were present at 7-8 of the airport properties; and ducks, herons, and rock doves were present at 
5-6. Questionnaires indicated that pilots using focal airports were accustomed to wildlife hazards: $69 \%$ of respondents reported that they had altered aircraft operation due to wildlife within the past year, and $25 \%$ reported involvement in a wildlife strike during the past year. Furthermore, $88 \%$ of respondents felt that wildlife populations at Indiana airports were at least "somewhat hazardous". Despite pilots' awareness of wildlife hazards, less than $70 \%$ of respondents supported the use of fencing or wildlife deterrents, $43 \%$ supported modification/elimination of wildlife habitat, and only $38 \%$ of respondents supported for lethal removal of wildlife on airport properties. Hazards associated with deer and coyotes can be alleviated by installing suitable fencing; for airports with extant fences, care should be taken to monitor fences regularly and repair gaps as soon as they are discovered. Presence of deer and coyotes inside airport fences should not be tolerated. Birds are best managed by maintaining airport habitats in a manner that minimizes availability and/or quality of food, water, cover, and loafing sites for hazardous species. Furthermore, several new technologies and refinements in techniques for wildlife damage management at airports have emerged recently and may benefit small airports, such as advancements in electric fencing and the use of dead bird effigies to repel some hazardous bird species.

Key words: wildlife, transportation, airstrike, roadkill, vertebrate, human/wildlife conflict 


\section{Introduction}

\section{TECHNICAL SUMMARY}

Conflicts between wildlife and human interests have increased in recent decades in Indiana due to growing human populations and the resulting expansion of anthropogenic pressures into wildlife habitats. One area of particular concern is vehicle-wildlife collisions. Such collisions often result in human injury and monetary losses, as well as high rates of mortality to many wildlife populations. In the research described herein, we assess the extent of vehicle-wildlife collisions in Indiana and evaluate management techniques designed to reduce them.

This project is divided into two Parts: Part I describes research on wildlife-automobile collisions; Part II describes research on wildlife-aircraft collisions. Both types of collisions are costly to Indiana residents in terms of economic losses and human injuries and deaths. Each Part is described separately in the context of the overall project.

Collisions between wildlife and automotive traffic (roadkill) can be a major source of mortality in animal populations. This is particularly troubling when the species impacted are of conservation concern. Animals are killed by traffic for a number of reasons, including the simple dispersal of juveniles for inbreeding avoidance (foxes), expansive home range size (bobcats), or migration to breeding sites (salamanders). Consider the potential impacts of roadkill on salamander population dynamics. Worldwide, amphibians populations are declining for unknown reasons. Migratory reptiles and amphibians such as tiger salamanders migrate to their breeding ponds en masse, often across state roads. Roadkill mortality of just 20 gravid females has the potential to remove hundreds if not thousands of salamanders from the local population because each female produces (on average) more than 1000 eggs.

Collisions between wildlife and aircraft (wildlife strikes) are a serious problem both for economic and safety reasons: the civil aviation industry incurs over $\$ 300$ million in damage from wildlife strikes every year, more than 100 people have died in the USA since 1960 in wildlife strikes, and over 350 people have been killed in wildlife strikes 
worldwide since the inception of aviation 100 years ago. Although mid-air collisions between aircraft and large soaring birds can be catastrophic, collisions in the airport environment are more problematic overall. Commercial and general aviation airports, which commonly are located in close proximity to water bodies and large grasslands, often harbor large populations of birds, white-tailed deer, coyotes, and other wildlife that are potentially dangerous to aircraft. The combination of abundant wildlife populations and frequent aircraft take-offs and landings at airports commonly leads to unacceptable levels of wildlife strike occurrences - over $90 \%$ of wildlife strikes to civil aircraft occur in the airport environment. Moreover, some researchers contend that the wildlife strike problem will continue to grow because several wildlife species that are regularly involved in wildlife strikes at airports are increasing in number across their natural ranges (e.g., white-tailed deer, gulls, Canada geese) due to the extirpation of top predators and continued habitat fragmentation. An increased understanding of the causal factors contributing to wildlife strikes at airports and the continued development of cost-effective solutions to reduce such collisions would potentially reduce human mortalities and substantial economic losses to the aviation industry.

\section{Findings}

There were several objectives for Part 1 (roadkill) of the proposed research. First, we reviewed and summarized the literature relevant to the mitigation of animal roadkill. This includes the vast primary scientific literature and the so-called "gray" literature (e.g., government documents). Second, we identified, characterized, and provided baseline data on 13 study sites that can be used as future evaluation sites. Finally, we include practical recommendations as to how state and federal agencies can implement wildlifefriendly engineering solutions (e.g., culverts).

The major findings of Part I include: 1) most roadkill occurs near wetlands; 2) both common and rare species, including those of state conservation concern, are impacted negatively by roadkill; 3) the types of animals killed by traffic varies considerably across seasons and years, largely in response to specific attributes of an organism's natural 
history (e.g., timing of migration); and 4) the taxonomic identification of animals killed by traffic can be difficult, but could be addressed easily with DNA analyses.

The objective for Part II (airstrike) was to evaluate the need for management actions to reduce wildlife strikes at general aviation airports throughout Indiana. In essence, we conducted a Wildlife Hazard Assessment at a subset of general aviation airports throughout Indiana. The research provides 1) a review of the wildlife strike literature, 2) an evaluation of wildlife hazards at 10 focal airports, and 3) recommendations for wildlife management actions at the 10 focal airports where wildlife hazards appear problematic.

The major findings of Part II include 1) at least two types of wildlife attractants were present at each airport property surveyed, but most airports had five to seven types including standing water (ephemeral), open culverts, crop fields, woodlot refugia and gravel pits, 2) the proportion of airport perimeter fenced ranged from $7.5 \%$ to $100 \%$, but most airports were $>40 \%$ fenced; however, most airports with $>25 \%$ perimeter enclosed by chain-link fencing had 0.2-0.5 openings per 100 meters of fence with dig holes and gaps being the most prevalent types of openings, 3) hazardous mammalian species observed included white-tailed deer and coyotes while hazardous avian species including American kestrel, blackbirds-starlings, crows-ravens, mourning doves, shorebirds, sparrows and swallows were observed at 9-10 airports; geese, hawks and vultures were observed at 7-8 airports; and ducks, herons and rock doves were present at 5-6 airports, 4) pilot questionnaires indicated that $69 \%$ of respondents had altered aircraft operation due to wildlife in the past year and $25 \%$ of respondents reported involvement in a wildlife strike in the past year; additionally, $88 \%$ of respondents felt that wildlife populations at Indiana airports were at least "somewhat hazardous". Our research demonstrated that despite the lack of published information concerning wildlife hazards at small airports, the potential for significant wildlife strikes at such sites in Indiana does exist. Our habitat assessments, wildlife surveys, and pilot questionnaires all indicated that more emphasis should be given to the problem of wildlife strikes by airport personnel at general aviation airports in Indiana. 


\section{Implementation}

With regard to Part I (roadkill), our data indicate that roadkill in Indiana is largely associated with wetlands. Thus, we recommend that engineers who are either renovating old roads or designing new ones near wetlands work with biologists to identify costeffective devices (e.g., wildlife-friendly culverts) that should reduce collisions between wildlife and vehicular traffic. In order to groundtruth these recommendations, we suggest INDOT/JTRP consider installing roadkill-mitigation devices at one or more of our study sites because the key baseline data (reported herein) can be used for comparative purposes.

With regard to Part II (airstrike), our data indicate that wildlife hazard management should be improved at all general aviation airports in Indiana. Because most wildlife hazard problems at airports can be addressed with traditional methods, we recommend that airport personnel become familiar with established techniques, such as those summarized in Cleary and Dolbeer (1999). Hazards associated with deer and coyotes could be alleviated by installing suitable fencing where funds are available. For airports with extant fences, care should be taken to monitor fences regularly and repair gaps as soon as they are discovered. Presence of deer and coyotes inside airport fences should not be tolerated. Birds are best managed by maintaining airport habitats in a manner that minimizes availability and/or quality of food, water, cover, and loafing sites for hazardous species. Furthermore, several new technologies and refinements in techniques for wildlife damage management at airports have emerged recently and may benefit small airports, and we suggest that INDOT/JTRP should investigate the utility of these new technologies - such as advancements in electric fencing and the use of dead bird effigies to repel some hazardous bird species. 


\section{TABLE OF CONTENTS}

CHAPTER 1. VERTEBRATE MORTALITY ON INDIANA ROADWAYS..........1

Executive Summary .....................................................

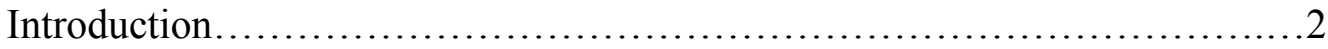

Wildlife/vehicle collisions..................................................

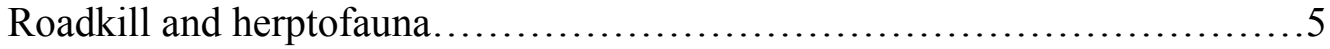

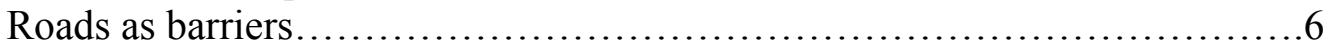

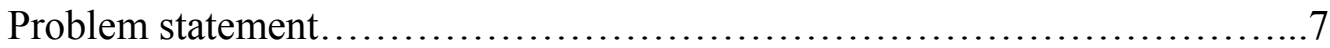

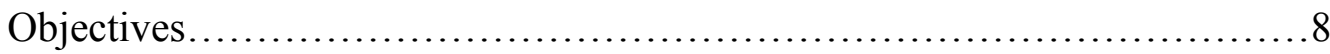

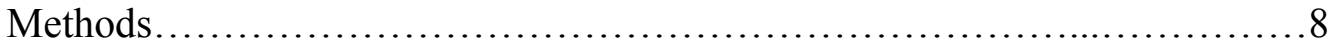

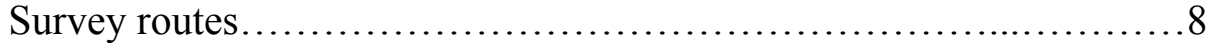

Roadkill sampling........................................... 9

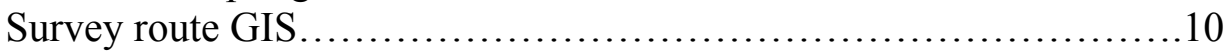

Habitat analyses................................................ 12

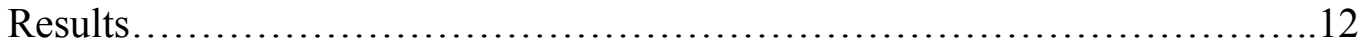

Roadkill sampling ..............................................

Weather and habitat analyses...................................... 14

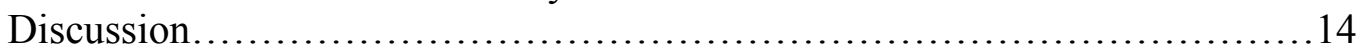

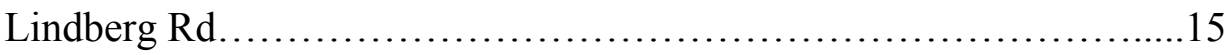

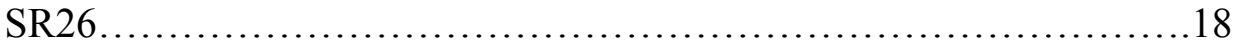

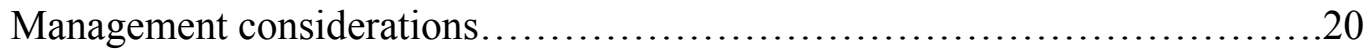

CHAPTER 2. MITIGATION MEASURES FOR REDUCING WILDLIFE

MORTALITY ON ROADWAYS: A REVIEW.................................52

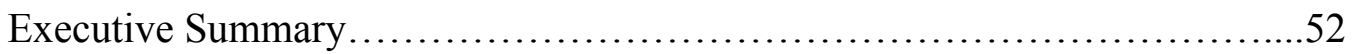

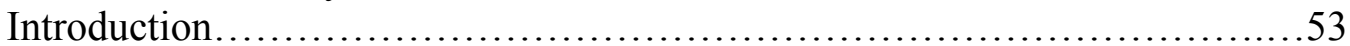

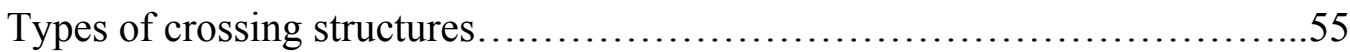

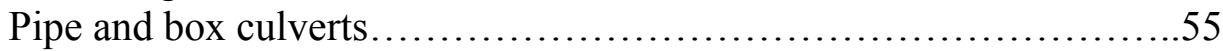

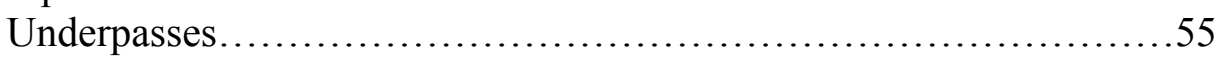

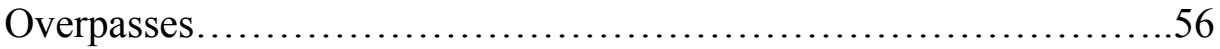

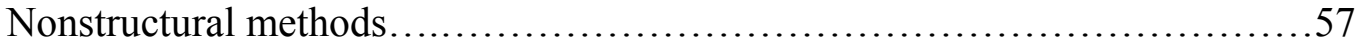

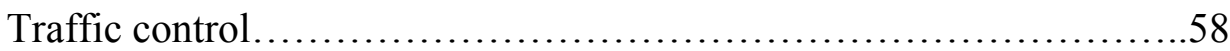

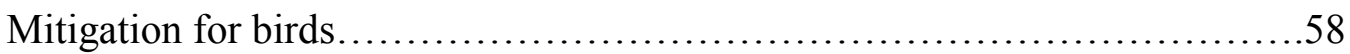

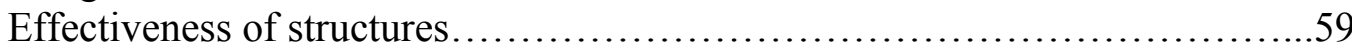

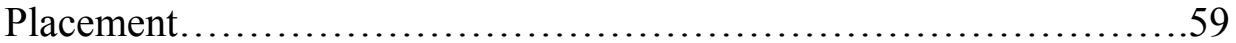

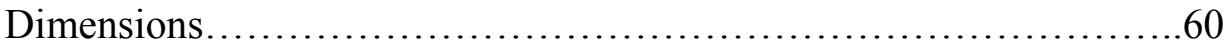

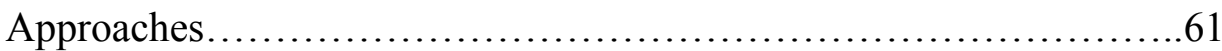

Fencing and directional devices.................................61

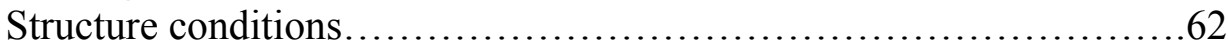

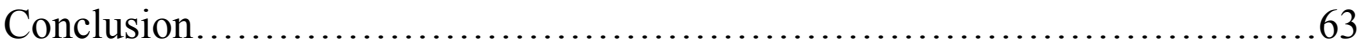

CHAPTER 3. EVALUATION OF WILDLIFE HAZARDS AT GENERAL AVIATION

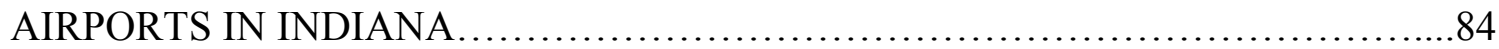

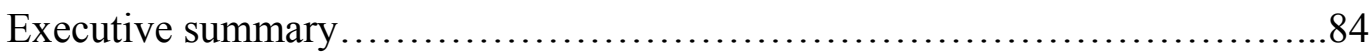

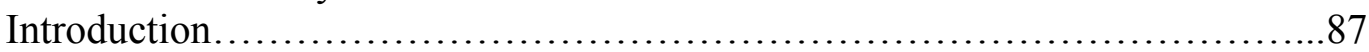

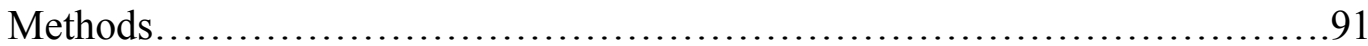

Selection of focal airports........................................ 91 
Wildlife inventories............................................ 91

Spotlight surveys...........................................91

Bird surveys...............................................92

Remote camera surveys.................................992

Opportunistic observations................................93

Habitat and fencing evaluations.................................... 93

On-site analyses..........................................93

GIS within airport boundaries.............................96

GIS outside airport boundaries................................97

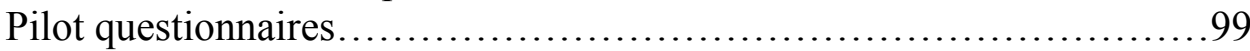

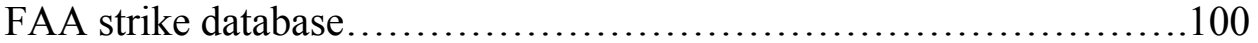

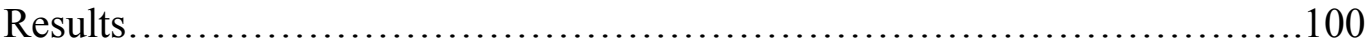

Wildlife inventories........................................... 100

Spotlight surveys........................................101

Bird surveys..............................................101

Remote camera surveys...................................101

Opportunistic observations...............................102

Habitat and fencing evaluations...................................102

On-site assessments and GIS within airport boundaries..........102

GIS outside airport boundaries.............................102

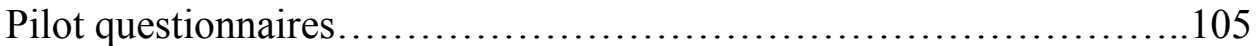

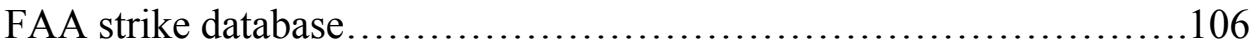

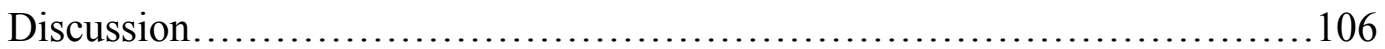

Wildlife inventories............................................. 107

Habitat and fencing evaluations.................................... 108

Pilot questionnaires.................................................. 112

FAA strike database ............................................ 112

Management recommendations............................................ 113 


\section{CHAPTER 1. VERTEBRATE MORTALITY ON INDIANA ROADWAYS}

\section{EXECUTIVE SUMMARY}

Conflicts between wildlife and human interests have increased in recent decades due to growing human populations and the resulting expansion of anthropogenic pressures into wildlife habitat. One area of particular concern is vehicle-wildlife collisions. Such collisions often result in human injury and monetary losses, as well as high rates of mortality to many wildlife species. Our overall objectives included quantifying vertebrate mortality on a variety of Indiana roadways, incorporating these empirical data into a GIS database to identify habitat characteristics of roads with high vertebrate mortality, reviewing/summarizing the current roadkill mitigation literature, and recommending mitigation approaches that may be most appropriate for Indiana roadways.

Survey routes were chosen using maps and through consultations with regional biologists; the 13 chosen routes represent a variety of geographic and anthropogenic conditions covering a total of 180 linear $\mathrm{km}$. Routes were divided into 2 categories, local and remote. Local routes were surveyed biweekly throughout the year while remote routes were surveyed over each of the 4 seasons. All carcass locations within the road shoulders were recorded using GPS and then marked or removed to avoid recounting. We recorded over 11,000 road mortality events across all 13 of our survey routes. At least 80 species were represented among the mortalities across all 13 routes and of these, $91 \%$ were herps, $7 \%$ mammals, and $2 \%$ birds. Of the $>11,000$ roadkills, 10,515 were detected on the local routes and were comprised of $95 \%$ herps, $3 \%$ mammals, and $2 \%$ birds. 
While roadkills were detected in all months, there were obvious seasonal and weather related patterns in the data. Most roadkills occurred from July through September which was concurrent with peak temperatures and precipitation levels. While habitat variables differed across all 4 local routes, water was a significant factor in high vertebrate mortality at both the Lindberg Rd and SR 26 routes.

\section{INTRODUCTION}

The expansion of humans into the wilderness is not a new phenomenon. Humans have been steadily intensifying their impact on the wild areas of the world, especially since the advent of large-scale automobile manufacturing in the early $20^{\text {th }}$ century. Areas that formerly saw only horse and wagon traffic are now inundated with automobiles, because many formerly remote places can now be accessed by the public. Moreover, the increase in personal vehicles required a well-developed road system and increased resource extraction which has led to increased contact and conflict between humans and wildlife. The 6.2 million km system of public roads, used by 200 million vehicles, links essentially every local area in the United States (National Research Council 1997). Road corridors, defined as the road surface plus its maintained roadsides and parallel vegetated strips, cover about $1 \%$ of the land, a combined area equivalent to South Carolina (Forman 2000).

Many ecological effects of roads on species, soils, and water have been identified, with effects varying in distance outward from meters to kilometers (Ellenberg et al. 1991, Forman 1995). These "road-effect zones" impact an estimated 15-20\% of the United States (Forman and Alexander 1998). While roads are an important part of the infrastructure and can provide some ecological benefits such as maintenance of grassland 
plants in intense agricultural areas (Forman 2000), they can also present several ecological problems. In instances where exotic plant species are planted along roads to help combat erosion, snow accumulation, and enhance aesthetics, they have the potential to spread into nearby natural ecosystems (Forman 1995). Runoff pollutants from roads (primarily deicing salts and heavy metals) can alter soil chemistry, be absorbed by plants, and affect stream ecosystems (Forman and Alexander 1998). Roads can also act as both physical and biological barriers to many wildlife species (Jackson 2000, Forman and Alexander 1998). Likewise, roads can be direct sources of wildlife mortality and in some instances, act as a predatory mechanism (Langton 1989).

\section{Wildlife/vehicle collisions}

Collisions with automotive traffic can be a major source of mortality in animal populations (Romin and Bissonette 1996, Trombulak and Frissell 2000, Gibbs and Shriver 2002). Lalo (1987) estimated vertebrate mortality on United States roads at 1 million individuals per day. For many species, road mortality can serve as a populationlimiting factor as their foraging and dispersal behaviors put them at risk of being struck on roadways. In Launceston, Australia, annual road mortality of the brushtail possum (Trichosurus vulpecula) exceeds the local birth rate (Statham and Statham 1997) and wildlife/vehicle collisions are the primary cause of death in moose (Alces alces) in the Kenai National Wildlife Refuge, Alaska (Bangs et al. 1989). Road mortality can be especially destructive to carnivores, which normally have low reproductive rates, low population densities, and large home ranges (Ruediger 1996). For example, road mortality is the third-highest cause of death for wolves in Minnesota (Fuller 1989). 
While roadkills may not affect abundant species, they can have a significant impact on populations of threatened or endangered species. Roadkills are a significant source of mortality for the endangered population of the eastern barred bandicoot (Perameles gunnii) in Victoria, Australia (Brown 1989) and are considered a major threat to the recovery and viability of the endangered Florida panther (Felis concolor coryi) (Foster and Humphrey 1995, Evink et al. 1996). Road mortality has also served as a limiting factor in the recovery of the American crocodile (Crocodylus acutus; Kushlan 1988) and as a contributor to the endangerment of the prairie garter snake (Thamnopsis radix radix) (Dalrymple and Reichenbach 1984).

From the human perspective, animal road mortality can pose both safety and economic issues. Collisions with animals can result in serious injury or even death to motorists. In addition, drivers may attempt to avoid animals on the road, subsequently endangering themselves and others. Groot Bruinderink and Hazebroek (1996) estimated the annual number of collisions with ungulates in Europe at 507,000 resulting in 300 human fatalities, 30,000 injuries, and $\$ 1$ billion (U.S.) in damages. An estimated 1.5 million animal-vehicle collisions involving deer (Odocoileus spp.) alone occur annually in the United States (Conover et al. 1995). Estimated damage to vehicles in these collisions exceeds $\$ 1.1$ billion in total and approximately $\$ 1,500$ per collision (Conover et al. 1995). Conover et al. (1995) reported that deer/vehicle collisions alone resulted in over 29,000 human injuries and over 200 fatalities in the United States. Overall, human injury results from approximately $4 \%$ of collisions involving medium-sized animals (Conover et al. 1995) and 14\%-18\% of collisions with larger animals such as moose 
(Farrell et al. 1996). These figures do not account for losses due to collisions with other wildlife and only represent reported animal-vehicle collisions.

\section{Roadkill and herpetofauna}

While many road mortality studies have centered on large carnivores and ungulates, the effects of roads and roadkill are also felt by many reptiles and amphibians (herpetofauna, herps). Over the last decade, amphibian populations have been declining worldwide (Blaustein and Wake 1990, Wake 1991, Fahrig et al. 1995) and these declines are often associated with some type of habitat modification such as fragmentation and/or road expansion (Fahrig et al. 1995, Vos 1997). In fact, even though amphibians may tend to avoid roads, a growing literature suggests that the greatest transportation impact on amphibians is mortality associated with roadkill (Fahrig et al. 1995, Ashley and Robinson 1996, Vos 1997). In Australia, Ehmann and Cogger (1985) estimated the annual mortality of herps on roads at 5 million individuals. Road mortality is especially prevalent in areas where roads intersect wetlands. In a 4 year period, Ashley and Robinson (1996) observed $>32,000$ individual animals (92\% amphibians) killed along a $3.6 \mathrm{~km}$ stretch of road in Ontario. Herpetofaunal road mortality can have significant impacts on populations, such as altering age and sex ratios, and can eventually lead to local extinctions (Langton 1989, Vos and Chardon 1998). Moreover, reduced reptile and amphibian populations may not have to range as far for resources, possibly affecting the distribution and abundance of the general plant and animal community (Langton 1989).

Another reason that road mortality is a key concern for herpetofauna can be found in their biology. Herps generally move slower than mammals and birds, making them more susceptible to road mortality. Furthermore, many herp species migrate during their 
breeding seasons. When amphibians must migrate across roads to reach breeding ponds, mortality of breeding adults can reach $20 \%-40 \%$ (Langton 1989). With female ambystomatid salamanders producing (on average) over 1,000 eggs per individual and anuran egg numbers ranging from several hundred (in smaller hylids) to several thousand (in larger ranids and bufonids) (Wright and Wright 1949, Harding 1997), the road mortality of migrating gravid females has the potential to remove tens or even hundreds of new salamanders, frogs, and toads from the population. The impact of road mortality on populations of reptile species such as snapping turtles (Chelydra serpentina) may be detrimental due to their inherent life-history traits, e.g., low annual recruitment and long life expectancy (Galbraith and Brooks 1987, Galbraith et al. 1989, Haxton 2000). Additionally, many reptile species bask during the warmth of the day to increase their body temperatures and metabolisms. This thermoregulatory behavior can bring reptiles into a position of imminent danger.

\section{Roads as barriers}

In many instances, roads not only serve as direct sources of mortality but also as barriers to wildlife species (Forman and Alexander 1998, Jackson 2000). For some species, such as grey wolves (Canis lupus) and pronghorn antelope (Antilocapra americana), movements and ranges can be limited by the presence of roads. Jensen et al. (1986) and Thurber et al. (1994) found that gray wolves will not establish themselves in areas with road densities above certain region-specific thresholds. Black bears (Ursus americanus) and grizzly bears (Ursus horribilis) regularly shift their home ranges away from areas of high road density (McClellan and Shackelton 1988, Brody and Pelton 1989) and pronghorn antelope likewise show an aversion to roads (Bruns 1977). 
Similarly, herp species are reluctant to cross roads (Fahrig et al. 1995) as they may represent both physical and biological barriers (Mader 1984). Gibbs (1998) found that roads significantly hindered amphibian movement.

The barrier effect of roads can also play an important role in the population structure and gene flow of a species. For example, turtle populations are susceptible to decline due to increased mortality of reproductive adults (Brooks et al. 1991). Turtle life history is characterized by low annual recruitment rates, high adult survival rates, and delayed sexual maturity (Congdon et al. 1993, 1994). Steen and Gibbs (2004) found that high road density was associated with male-biased populations of painted turtles (Chrysemys picta) and snapping turtles, primarily due to the disproportionate level of road mortality among breeding females. Reh and Seitz (1990) found significantly lower levels of genetic heterozygosity and polymorphism among populations of common frogs (Rana temporaria) separated by roads in Germany.

\section{PROBLEM STATEMENT}

Indiana is composed primarily of a highly fragmented, agriculturally dominated landscape consisting of over 150,000 km of roads, with the Indiana Department of Transportation (INDOT) managing $\sim 17,000 \mathrm{~km}$ of these (INDOT website). The biological effects of this road network are not well-understood; however, the combination of high habitat fragmentation and high road density may have detrimental effects on many wildlife species including herpetofauna. Presently, outside of raccoon roadkill surveys (for population estimates) conducted by the Indiana Department of Natural Resources (IDNR), there is no other annual roadkill survey nor is there any multi-species road mortality index for the state. 
Wildlife road mortality is a serious issue both ecologically and sociologically. As scientists, we have an obligation to try to understand these issues and recommend responsible solutions. Understanding the effects of roadways on herpetofauna and other species of wildlife can help reduce road mortality and identify mitigation solutions for “conservation engineers" and public policy makers.

\section{OBJECTIVES}

Our research had 2 objectives: 1) identify, characterize, and evaluate roadkill sites throughout Indiana and incorporate these empirical data into a Geographical Information System (GIS) database to identify affected species and habitat characteristics of roads with high vertebrate mortality (with an emphasis on herpetofauna) and 2) review and summarize the current roadkill mitigation literature and offer suggestions to INDOT as to which measures may be most appropriate for Indiana roadways. Two questions that we hoped to address through analyses of these data are: (1) which habitat types have the greatest influence on road mortality, and (2) which species are most often encountered as roadkill?

\section{METHODS}

\section{Survey Routes}

Identifying potential roadkill survey routes throughout Indiana was done using physical maps and consultations with regional biologists. We primarily focused on state and U.S. roads as these are under more direct control of INDOT. Survey routes varied in length and were chosen to represent a mixture of geographic and anthropologic conditions (e.g., upland vs. wetland, rural vs. suburban) (Tables 1 and 2). Survey routes were also chosen based on safety and accessibility (e.g. available shoulder, visibility). 
Overall, 13 survey routes ( 4 local, 9 remote) were selected across the state covering a total of 180 linear km (Figures 1 and 2). Local survey routes were located close to Purdue and $<5 \mathrm{~km}$ in length, to allow for more thorough sampling over a longer period of time. For logistical reasons, remote sites were determined in conjunction with the statewide wildlife/aircraft collision study conducted by Purdue University and were generally $>10 \mathrm{~km}$ in length to allow for more road sampling per trip.

\section{Roadkill Sampling}

Roadkill detection surveys were performed on all selected routes. Routes were driven at low speeds $(<40 \mathrm{~km} / \mathrm{h})$ to allow for better detection of roadkills. In some instances, routes were partially or entirely walked for safety reasons (e.g., lack of a vehicle-accessible shoulder) and/or amounts of kills in a particular area. Local routes were surveyed biweekly from March 8, 2005 - July 31, 2006 for a total of 124 surveys per route. This intensive sampling of local routes promoted better and more frequent detection of smaller carcasses, e.g., tiger salamanders (Ambystoma tigrinum) during breeding migrations. We sampled each remote route twice during each of 4 seasons (spring 2005, summer 2005, fall 2005, and winter 2006) for a total of 8 visits per route in conjunction with the wildlife/aircraft collision schedule. Surveys accounted for all carcasses found within the road shoulders. All roadkills were identified to species (whenever possible), marked or removed to avoid recounting, and their locations were entered into a Trimble GeoXT mobile GPS/GIS system. Removed carcasses in excellent condition were donated to the vertebrate collection at Purdue University.

Because of the low speeds necessary for effective surveying and surveyors' close proximity to roads when marking individual carcasses, safety was a top priority. Safety 
orientation was completed via an on-line video from the Joint Transportation Research Program at Purdue. All surveying vehicles were equipped with amber beacons and flashers and any surveyor exiting the vehicle along a route was required to wear a high visibility safety vest. In addition, signs were posted when necessary to alert oncoming traffic of surveying activity.

\section{Survey Route GIS}

Using ArcGIS9, a database was developed for all 13 survey routes. We referred to aerial photographs obtained from the Indiana Spatial Data Service (http://www.indiana.edu/ gisdata/) to aid in interpretation of the spatial extent and location of habitat patches. The sid. file for each raster download was added to an ArcMap project and served as a base map for digitizing survey route buffers and habitat types. We applied a $100 \mathrm{~m}$ buffer (from the center of the road) parallel to each survey route and overlaid it onto its corresponding aerial photo. The $100 \mathrm{~m}$ buffer was chosen as it would include the critical habitat immediately adjacent to each survey route. Moreover, habitat management and mitigation measures would be more likely implemented nearer to the road. We created 8 land cover feature classes and then digitized those features within the buffer based on my interpretation of landscape features visible on the aerial imagery. Land cover feature classes included roads, grass/shrub ditches, agriculture/pasture, forest/woodlot, urban/recreational grasses, urban/residential, water/wetlands, and grassland/shrublands. We defined roads as the amount of paved roads within the buffer. Grass/shrub ditches consisted of roadside and field drainages. Agriculture/pasture habitat was defined as areas containing row-crop fields, hay and alfalfa, and pasture areas for livestock. We defined forest/woodlot habitat as areas 
consisting of wooded areas having a closed canopy. Urban/recreational grass areas were defined by large, maintained open areas that were obviously used routinely (e.g., golf courses and athletic fields) excluding lawns immediately surrounding residences. Urban/residential habitat consisted of developed areas (including lawns). We defined water/wetlands as areas of open water, streams, swamps, and bogs. Grassland/shrubland habitat consisted of areas of grasses that did not appear to be pasture, lawn, recreational field, or wetland and non-wetland habitat consisting of a mix of grass, shrubs, and young trees. Following completion of digitizing habitat classes, we used the Calculate Area tool in ArcMap to determine the area $\left(\mathrm{m}^{2}\right)$ of each habitat polygon per 100m section. Polygon areas were then summed for each habitat class and converted to a percentage per road section.

Other data collected for each route were presence/ absence of culverts, underpasses, and overpasses, road aspect (straight or curved), number of lanes, width, and posted speed limits (Table 3). Traffic volume data for survey routes was acquired through INDOT but since it was not consistent (results and updates were from different years) it was excluded from the habitat analysis. Weather data was obtained from the Indiana State Climate Office (http://shadow.agry.purdue.edu/sc.index.html) and we calculated the monthly mean temperature and monthly total precipitation levels for the analysis. Roadkill location data points were downloaded to the computer using TerraSync and GPS Pathfinder Office software (Trimble 2003) then projected on their respective routes. Each route along with its buffer was divided into $100 \mathrm{~m}$ sections (Smith and Dodd 2003) essentially constructing a 100m x 200m analysis "window" from 
which the number of roadkills and the corresponding habitat composition and road characteristics within each section could be determined (Figures 3, 4, 5, 6).

\section{Habitat Analysis}

At present, habitat analyses have been conducted for the local routes $(n=4)$ only as they were sampled for roadkill the most frequently. Moreover the road characteristics (e.g. aspect, speed limits) were generally homogenous for each route; therefore they were excluded from the analyses. Therefore, we examined the spatial distribution of road mortality events and its intensity along all local routes to determine which habitat variable most influenced roadkill numbers. Each section on a route represented one sampling unit with the response variable being the number of roadkills and the predictor variables consisting of the aforementioned habitat variables. Using ArcMap, we were able to determine the exact number of kills and the composition of the habitat per section. We then used SAS 9.1 (SAS Institute 2002) to perform stepwise linear regressions to determine which habitat variables were the best predictors of roadkill for each route. The data was log-transformed in instances of assumption violation. Furthermore, residuals of highly correlated habitat variables were used in the regressions rather than excluding them. This allowed us to still determine which habitat types were most significant in predicting roadkill. Road mortality data also was indexed to determine which species are most often killed along the survey routes.

\section{RESULTS}

We have chosen to report and discuss results primarily from the more intensive local surveys due to differences in the sampling regimes and data sets between the local and remote routes. 


\section{Roadkill Sampling}

From March 8, 2005-July 31, 2006, We recorded 11,068 road mortality events across all 13 survey routes (Table 4). A total of 72 surveys encompassing $1268 \mathrm{~km}$ were conducted across 9 remote routes while 496 surveys traveling a total of $1488 \mathrm{~km}$ were conducted at the local routes $(n=4)$. At least 80 species were represented among the mortalities across all 13 routes and of these, $91 \%$ were herps, $7 \%$ mammals, and $2 \%$ birds (Table 5). For the remote routes, the most common herp, mammal, and bird species were painted turtles (Chrysemys picta, $\mathrm{n}=12$ ), opossums (Didelphis virginiana, $\mathrm{n}=179$ ) and raccoons (Procyon lotor, $\mathrm{n}=134)$, and American robins (Turdus migratorius, $\mathrm{n}=9$ ) respectively. The remote routes with the highest incidence of roadkill were the DeKalb route $(\mathrm{n}=133 ; 8.3 \%$ herps, $88 \%$ mammals, $3.7 \%$ birds $)$ and the Richmond route $(\mathrm{n}=72$; $16.7 \%$ herps, $68 \%$ mammals, $15.3 \%$ birds).

Of the $>11,000$ roadkills, 10,515 were detected on the local routes and were comprised of $95 \%$ herps, $3 \%$ mammals, and $2 \%$ birds (Table 6 ). The most common herp species at the local level were bullfrogs (Rana catesbeiana, $\mathrm{n}=1,671)$, green frogs (Rana clamitans, $\mathrm{n}=172$ ), and tiger salamanders (Ambystoma tigrinum, $\mathrm{n}=142)$. The most frequent mammal species were opossums $(n=79)$ and raccoons $(n=43)$. Chimney swifts (Chaetura pelagica, $n=36)$ and American robins $(n=18)$ represented the highest numbers of avian roadkill.

The local routes with the highest incidence of roadkill were Lindberg $\operatorname{Rd}(\mathrm{n}=$ 8,176 ) with herpetofauna representing $98 \%$ of all roadkills, mammals $0.9 \%$, and birds $1.1 \%$ and the SR 26 route $(\mathrm{n}=1,761)$ of which $94 \%$ were herps, $4.1 \%$ mammals, and $1.9 \%$ birds. The kill total for the US 231 route was 346 , with $67 \%$ herps, $23 \%$ mammals, 
and $10 \%$ birds. South River Rd totaled 232 roadkills of which $21 \%$ were herps, $57 \%$ were mammals, and $22 \%$ were birds.

\section{Weather and Habitat Analysis}

Average monthly temperatures and monthly precipitation totals were plotted against total monthly roadkill numbers for all local routes (Figure 7). While roadkills were detected in all months, there were obvious seasonal and weather related patterns in the data. Most roadkills occurred from July through September which was concurrent with peak temperatures and precipitation levels. A similar pattern was witnessed at each individual local route (Figures 8, 9, 10, 11).

A summary of the results of habitat variable effects on roadkill numbers for each local route can be found in Table 7 . For the SR 26 route, water/wetland and forest habitats were significant predictors of roadkill numbers. Grass/shrub ditch habitat was a significant predictor of roadkill numbers along the South River Rd route. Along the US 231 route, urban/residential habitat had the biggest influence on roadkill. For the Lindberg Rd route, 5 of the 6 habitat types (urban/recreational, water/wetlands, urban/residential, forest/woodlot, and roads) found within the $100 \mathrm{~m}$ buffer were all found to be significant predictors of roadkill numbers.

\section{DISCUSSION}

While there have been other road mortality studies conducted throughout the world, there have been no similar ones in Indiana so, unfortunately, there are no other records with which to compare our results. Ashley and Robinson (1996) surveyed a $3.6 \mathrm{~km}$ section of road that bisected a wetland in Ontario, Canada over two 2-year periods and recorded $>32,000$ road mortalities. Of those $95 \%$ were reptiles and amphibians. In 
one year, Smith and Dodd (2003) counted $>1,800$ mortalities along a $3.2 \mathrm{~km}$ section of highway in Florida and of those 91\% were herpetofauna. There are obvious differences in the Ontario and Florida studies when compared to ours, most notably the scope. Where these prior studies focused on a single stretch of road, we looked at multiple road segments both close to Purdue University and throughout the state. Even though this led to a reduced number of surveys for the state-wide routes, the intensive local surveys produced very similar herpetofauna roadkill numbers (all local routes $=95 \%$ ) to the aforementioned studies. Herpetofaunal mortalities also compare favorably to the Ashley and Robinson and Smith and Dodd studies; Lindberg Rd (98\%) and SR 26 (94\%).

Live animals were not included in the data set, but general observations of live animals in the presence of the survey routes were often noted. Canada geese (Branta canadensis) were often seen milling about at both the Lindberg Rd and SR 26 routes. In fact, geese were so prevalent at Lindberg Rd during the spring and summer that they often caused traffic to stop while they crossed the road (Figure 12).

\section{Lindberg Rd}

The Lindberg Rd survey route, by way of its large number of amphibian road mortality, became a focal point of the study (Figure 14). Lindberg Rd, located in West Lafayette, bisects the Celery Bog Nature Preserve. The bog is surrounded by human development of various types; a golf course, shopping center, apartment complexes, and residential subdivisions. Prior to development, the habitat surrounding the bog was mostly agricultural fields. The road through the bog is a commonly used thoroughfare for commuters and college students and a convenient way to get from the western end of the Purdue campus to many West Lafayette amenities. 
Beginning in March 2005 we recorded >7,900 frogs (Rana sp.) killed on this $1.8 \mathrm{~km}$ stretch of road. These may be underestimates, as many individual herp specimens were only identifiable to genus due to carcass deterioration, especially during the summer months. For example, over $50 \%$ of the mortalities were frogs from the genus Rana including the northern leopard frog (Rana pipiens), a species of special concern in Indiana, but not all frogs could be identified to species. Twenty-nine leopard frogs were found on the road over the course of the study and several were heard calling from the bog area during early spring surveys. Bullfrogs were the most frequently recorded, identifiable frog species. They also were the most often species heard and seen alive in the bog. Bullfrogs are prolific breeders, often laying several thousands of eggs per female (Wright and Wright 1949, Harding 1997). This may explain the large numbers of bullfrogs that were recorded on the road. They are also voracious predators that will not only out-compete other species but also prey on them which may also explain the disproportionate number of bullfrogs to other frog species. Many of the frogs identified as Rana spp. may indeed have been bullfrogs but that could not be determined with any certainty.

While anurans made up the bulk of road mortality on Lindberg Rd, there were some other notable mortality events. For example, between 17 February 2006 and 7 April 2006 we recorded 30 tiger salamanders during their spring migration to breeding areas and during a 46 day period between April and June 2006, 34 chimney swifts, most likely migrants, were found dead on the route. Most swift carcasses were located on the sections of road bisecting the bog and were probably a result of low-flying birds striking vehicles while pursuing insects (Figure 13). While these numbers may not seem large, 
consider that up to these points in time neither species had been recorded during prior surveys. If migrating animals use the bog as a stopover or breeding area they could potentially be put in a position of imminent danger.

The Lindberg Rd habitat analysis model found 5 of the 6 habitat types present (urban/recreational grasses, water/wetlands, urban/residential, forest/woodlot, and roads) to be significant roadkill predictors and this is most likely due to the high number and distribution of kills over the majority of the route. Water was one of the significant factors when determining the number of roadkills per 100m section of road and this makes sense when considering the high numbers of herps that were recorded along those routes. The bog notwithstanding, there are several other sources of water such as apartment complex retention ponds and golf course water "hazards" that could potentially be used by various herp species as breeding, cover, and feeding areas. This in fact could be the reason that herp carcasses were found along the entire route.

As with the other local routes, weather and season were an influence on roadkill numbers. Roadkills along the route were highest during the late summer months and peaked in September. The mortality patterns of amphibians in response to seasonal changes can be explained by the life histories of the various species recorded. There were several newly metamorphosed subadult carcasses recorded during this period which may be due to dispersal and may have inflated numbers (Figure 15). Ashley and Robinson (1996) recorded the monthly road mortalities of 4 species of anurans (northern leopard frogs, bullfrogs, green frogs, and American toads) and discovered distinct patterns for each species. Leopard frog mortalities were unimodal with the peak being in late summer. Bullfrog, green frog, and American toad mortalities were bimodal having 
peaks both in mid-spring and late summer. Smith and Dodd (2003) discovered similar patterns in their roadkill and weather/season data. They recorded high kill rates for frogs in July and August and an overall higher number of roadkills throughout the summer months.

\section{SR 26}

The SR 26 route is a 2-lane highway located in rural western Tippecanoe County. The route was chosen because of a number of tiger salamanders that were discovered killed on the road during their spring breeding migration 2 years prior to our study and indeed were found again $(n=106)$. The overall number of roadkills along the SR 26 route $(n=1761)$ were considerably less than that of the Lindberg Rd route. Nevertheless, it is still quite spectacular considering the amount of time the route was sampled. Again, bullfrogs were the predominant herp species but there was a higher overall diversity of herp species $(n=16)$ found along this route than any other local route. This may be because of the presence of all 8 habitat types within the $100 \mathrm{~m}$ buffer and their ability to support a variety of species. As with the Lindberg Rd route, water was a significant factor in predicting roadkills. There is a large wetland located in the center and immediate south of the road with upland forested habitat directly across from it. This mix of upland and water is probably the reason tiger salamanders were killed along that stretch of road as it provides both breeding and over-wintering areas. Another similarity to Lindberg Rd is the multiple sources of water along the route in the form of farm ponds and creeks. Green frogs preferred the sections near the creeks while bullfrogs were prevalent in areas near the ponds and wetland. As mentioned before, weather and season 
were an influence on roadkill numbers. Roadkills along the route were highest during the summer months but unlike Lindberg Rd peaked in July.

Several issues arose during our project which may be pertinent to any similar future or follow-up projects. Sampling during the first year did not begin until March therefore many of the early salamander and anuran migrations may have been missed. However, during the second year of sampling we were able to document several early migrations such as tiger salamanders and northern leopard frogs. Detection and positive identification of carcasses could be extremely taxing for the surveyors. Small species such as spring peepers were very hard to locate and were undoubtedly missed on occasion. Carcass degradation, especially for herps during the summer months, made identification difficult. Moreover, some carcasses may have been eaten by scavengers prior to marking and some animals may have left the roadside after being hit. Infrequency of remote surveys did not allow for a true measure of yearly roadkill numbers along those routes and certainly contributed to a reduced number of recorded herpetofauna. In addition, these differences made comparing results between the two difficult. Weather can always be a factor and on days of bad weather visibility was extremely limited. Lastly, nature in its own right can be somewhat fickle meaning what is true today may not be tomorrow. At the time of this report, herpetofauna roadkill numbers on Lindberg Rd and SR 26 were down when comparing 2005 and 2006 numbers and the number of live anurans noticed either audibly or visually by surveyors also had decreased. This raises new questions about the high levels of roadkill of 2005 . Was 2005 simply a "blip" on the roadkill radar or are there other environmental and biological factors lending to the perceived decrease in herp activity? 


\section{MANAGEMENT CONSIDERATIONS}

Based on the results presented here, there are some obvious areas of wildlife road mortality that need attention most notably areas that have roads bisecting or in close proximity to wetlands. Connectivity of habitat and permeability of road systems are important factors to consider when developing roadkill mitigation systems.

Unfortunately, there is no panacea for mitigating roadkill; what works for one species or suite of species may not be the best option for others. There are, however, various measures that may be more effective for the areas of highest road mortality (i.e. Lindberg Rd and SR 26) many of which are discussed in the mitigation literature review section of this report. To reiterate, we will mention some of the more feasible possibilities here.

Wildlife underpasses, also known as wildlife bridges, are large underpasses that provide a relatively unconfined passage for wildlife (Jackson and Griffin 2000). Where roads cross over water or other roads, bridges can provide a passageway for many wildlife species, especially those that use riparian corridors. In situations where underpasses may hold excessive amounts of water, ledges can be incorporated into the designs to allow animal passage.

Pipe culverts are relatively small structures (1-6ft. diameter) made of concrete, smooth steel, or corrugated metal designed to carry water under roads. Europe has led the way in implementing smaller pipe-style culverts, also referred to as "amphibian tunnels" (Forman et al. 2003). Box culverts, generally larger than pipe culverts, are also used to allow water to pass under roads but unlike pipe culverts usually remain dry except in periods of heavy run off. Culverts may be used by a variety of wildlife species (Rodriguez et al. 1996, Yanes et al. 1995, Clevenger and Waltho 2000). Kaye et al. 
(2005) reported that the use of a box culvert under a highway improvement project in Massachusetts allowed the crossing of spotted turtles (Clemmys guttata, a state threatened species) between two turtle habitats. Clevenger et al. (2001) monitored 36 culverts along the Trans-Canada highway and found a total of 618 crossings by a minimum of 9 species, with an average of 2.8 species at each culvert. In Australia, Taylor and Goldingay (2003) recorded 17 different vertebrate species using purpose-built fauna culverts in combination with exclusion fence under the Pacific Highway. The use of a culvert system in conjunction with a barrier wall reduced roadkill numbers along US 441 in the Paynes Prairie State Preserve, Florida, by 93.5\% (Dodd 2004). The highway bisects a wetland complex and prior to construction and during a 1-year roadkill survey 2,411 roadkills were detected. That number was reduced to 158 animals after construction of the barrier wall/culvert system.

Systems similar to these may be effective for areas of high road mortality in Indiana such as Lindberg Rd or SR 26. They would allow road permeability, promote habitat connectivity and may be able to be instituted by modifying existing culverts and underpasses rather than building new ones. Mitigation systems should be taken into consideration during the planning stage of new road construction thereby factoring in the cost upfront. However, mitigation measures need to be placed in areas where they will be most effective; therefore additional road mortality studies such as this one need to be implemented in other parts of the state to verify additional roadkill hotspots. Moreover consistent post-construction monitoring will be needed to verify mitigation effectiveness and maintain structures. 


\section{REFERENCES}

Ashley, E. P., and J. T. Robinson. 1996. Road mortality of amphibians, reptiles and other wildlife on the Long Point Causeway, Lake Erie, Ontario. The Canadian Field-Naturalist 110:403-412.

Bangs, E. E., T. N. Bailey, and M. F. Portner. 1989. Survival rates of adult female moose on the Kenai Peninsula, Alaska. Journal of Wildlife Management 53:557-563.

Blaustein, A. R. and D. B. Wake. 1990. Declining amphibian populations: a global phenomenon? Trends in Ecology and Evolution 5:203-204.

Brody, A. J. and M. R. Pelton. 1989. Effects of roads on black bear movements in western North Carolina. Wildlife Society Bulletin 17:5-10.

Brooks, R. J., G. P. Brown, and D. A. Galbraith. 1991. Effects of a sudden increase in natural mortality of adults on a population of the common snapping turtle (Chelydra serpentina). Canadian Journal of Zoology 69:1314-1320.

Brown, P. R. 1989. Management plan for the conservation of the eastern barred bandicoot Perameles gunnii in Victoria. Arthur Rylah Institute for Environmental Research Technical Report Series No.63.

Bruns, E. H. 1977. Winter behavior of pronghorns in relation to habitat. Journal of Wildlife Management 41:560-571.

Clevenger, A. P., B. Chruszcz, and K. Gunson. 2001. Drainage culverts as habitat

linkages and factors affecting passage by mammals. Journal of Applied Ecology 38:13401349.

Clevenger, A. P., M. McIvor, D. McIvor, B. Chruszcz, and K. Gunson. 2001. Tiger salamander, Ambystoma tigrinum, movements and mortality on the TransCanada Highway in southwestern Alberta. The Canadian Field-Naturalist 115:199-204.

Clevenger, A. P., and N. Waltho. 2005. Performance indices to identify attributes of highway crossing structures facilitating movement of large mammals. Biological Conservation 121:453-464.

Congdon, J. D., A. E. Dunham, and R. C. Van Loben Sels. 1993. Delayed sexual maturity and demographics of Blanding's turtles (Emydoidea blandingii): implications for conservation and management of long-lived organisms. Conservation Biology 7:826-833.

Congdon, J. D., A. E. Dunham, and R. C. Van Loben Sels. 1994. Demographics of common snapping turtles (Chelydra serpentina): implications for conservation and management of long-lived organisms. American Zoologist 34:397-408. 
Conover, M. R., W. C. Pitt, K. K. Kessler, T. J. DuBow, and W. A. Sanborn. 1995. Review of human injuries, illness, and economic losses caused by wildlife in the United States. Wildlife Society Bulletin 23:407-414.

Dalrymple, G. H. and N. G. Reichenbach. 1984. Management of an endangered species of snake in Ohio, USA. Biological Conservation 30:195-200.

Dodd Jr., C. K. W. J. Barichivich, L. L. Smith. 2004. Effectiveness of a barrier wall and culverts in reducing wildlife mortality on a heavily traveled highway in Florida. Biological Conservation 118:619-631.

Ehmann, H., and H. Cogger. 1985. Australia's endangered herpetofauna: a review of criteria and policies. In Biology of Australasian frogs and Reptiles, G. Grigg, R. Shine, and H. Ehmann, eds. Surrey, Beatty, and Sons, New South Wales, Australia.

Ellenberg, H., K. Muller, and T. Stottele. 1991. Strassen-Okologie. Pp 19-115 in Okologie und strasse. Broschurenreihe de Deutschen Strassenliga, Bonn, Germany.

Evink, G. L., P. Garrett, D. Zeigler, and J. Berry (eds). 1996. Trends in addressing transportation related wildlife mortality. No. FL-ER-58-96. Florida Department of Transportation, Tallahassee, Florida, USA.

Fahrig, L., J. H. Pedlar, S. E. Pope, P. D. Taylor, and J. F. Wegner. 1995. Effect of road traffic on amphibian density. Biological Conservation 73:177-182.

Farrell, T. M., J. E. Sutton, D. E. Clark, W. R. Horner, K. I. Morris, K. S. Finison, G. E. Menchen, and K. H. Cohn. 1996. Moose-motor vehicle collisions. An increasing hazard in northern New England. Archives of Surgery 131:377381.

Forman, R. T. T. 1995. Land mosaics: the ecology of landscapes and regions. Cambridge University Press, Cambridge, United Kingdom.

Forman, R. T. T. 2000. Estimate of the area affected ecologically by the road system in the United States. Conservation Biology 14:31-35.

Forman, R. T. T., and L. E. Alexander. 1998. Roads and their major ecological effects. Annual Review of Ecology and Systematics 29:207-231.

Forman, R. T. T., D. Sperling, J. A. Bissonette, A. P. Clevenger, C. D. Cutshall, V. H.Dale, L. Fahrig, R. France, C. R. Goldman, K. Heanue, J. A. Jones, F. J.Swanson, T. Turrentine, and T. C. Winter. 2003. Road ecology; science and solutions. Island Press, Washington D. C., USA.

Foster, M. L., and S. R. Humphrey. 1995. Use of highway underpasses by Florida panthers and other wildlife. Wildlife Society Bulletin 23:95-100. 
Fuller, T. 1989. Population dynamics of wolves in north-central Minnesota. Wildlife Monographs 105:1-41.

Galbraith, D. A., and R. J. Brooks. 1987. Survivorship of adult females in a northern population of snapping turtles, Chelydra serpentina. Canadian Journal of Zoology 65:1581-1586.

Galbraith, D. A., R. J. Brooks, and M. E. Obbard. 1989. The influence of growth rate on age and body size at maturity in female snapping turtles (Chelydra serpentina). Copiea 1989:896-904.

Gibbs, J. P. 1998. Amphibian movements in response to forest edges, roads, and streambeds in southern New England. Journal of Wildlife Management 1998: 584-589.

Gibbs, J. P., and G. Shriver. 2002. Estimating the effects of road mortality on turtle populations. Conservation Biology 16:1647-1652.

Groot Briunderink, G. W. T. A. and E. Hazebroek. 1996. Ungulate traffic collisions in Europe. Conservation Biology 10:1059-1067.

Harding, J. H. 1997. Amphibians and reptiles of the Great Lakes Region. The University of Michigan Press, Ann Arbor, Michigan, USA.

Jackson, S D. 2000. Overview of transportation related wildlife problems. G. L. Evink, P. Garrett, and D. Zeigler. Proceedings of the Third International Conference on Wildlife Ecology and Transportation; Missoula, MT. Tallahassee, FL: Florida Department of Transportation.

Jackson, S. D., and C. R. Griffin. 2000. A strategy for mitigating highway impacts on wildlife. pp. 143-159 In Messmer, T. A., and B. West (eds) Wildlife and highways: seeking solutions to an ecological and socio-economic dilemma. The Wildlife Society.

Jensen, W. F., T. K. Fuller, and W. L. Robinson. 1986. Wolf, Canis lupus, distribution on the Ontario-Michigan border near Sault Ste. Marie. Canadian Field Naturalist 100:363-366.

Kaye, D., R., J., K. M. Walsh, and C. C. Ross. 2005. Spotted turtle use of a culvert under relocated Route 44 in Carver, Massachusetts. pp. 426-432 In 2005 ICOET Proceedings.

Kline, N. C., and D. E. Swann. 1998. Quantifying Wildlife Road Mortality in Saguaro National Park. Pages 23-27 in G. L. Evink, P. Garrett, D. Zeigler, and J. Berry (eds.) Proceedings of the international conference on wildlife ecology and transportation. Florida Department of Transportation, Tallahassee, Florida.

Kushlan, J. A. 1988. Conservation and management of the American crocodile. 
Environmental Management 12:777-790.

Lalo, J. 1987. The problem of roadkill. American Forests 50:50-52.

Langton, T. E. S. 1989. Reasons for preventing amphibian mortality on roads.

Proceedings of the Toad Tunnel Conference; Rendsburg, Germany. ACO Polymer Products Ltd, Bedfordshire, England.

Mader, H. J. 1984. Animal habitat isolation by roads and agricultural fields. Biological Conservation 29:81-96.

Malo, J. E., F. Suárez, and A. Díez. 2004. Can we mitigate animal-vehicle accidents using predictive models? Journal of Applied Ecology 41:701-710.

Mazerolle, M. J., M. Huot, and M. Gravel. 2005. Behavior of amphibians on the road in response to car traffic. Herpetologica 61:380-388.

McClellan, B. N. and D. M. Shackleton. 1988. Grizzly bears and resource-extraction industries: effects of roads on behavior, habitat use, and demography. Journal of Applied Ecology 25:451-460.

National Research Council. 1997. Toward a sustainable future: addressing the long-term effects of motor vehicle transportation on climate and ecology. National Academy Press, Washington, D.C., USA.

Reh, W. and A. Seitz. 1990. The influence of land use on the genetic structure of populations of the Common Frog Rana temporaria. Biological Conservation 54: $239-249$.

Rodriguez, A., G. Crema, and M. Delibes. 1997. Use of non-wildlife passages across a high-speed railway by terrestrial vertebrates. Journal of Applied Ecology $33: 1527-1540$.

Romin, L.A., and J. A. Bissonette. 1996. Temporal and spatial distribution of highway mortality of mule deer on newly constructed road at Jordanelle Reservoir, Utah. Great Basin Naturalist 56:1-11.

Rosen, P. C., and C. H. Lowe. 1994. Highway mortality of snakes in the Sonoran Desert of southern Arizona. Biological Conservation 68:143-148.

Ruediger, B. 1996. The relationship between rare carnivores and highways. In: Evink, G. L., P. Garrett, D. Zeigler, and J. Berry, eds. Proceedings of the transportation related wildlife mortality seminar (FL-ER-58-96). Florida Department of Transportation, Tallahassee, Florida, USA.

SAS 2002. SAS software, version 9.1. SAS Institute Inc., Cary, North Carolina, USA.

Smith, L. L., and C. K. Dodd Jr. 2003. Wildlife mortality on highway US 441 across 
Paynes Prairie, Alachua County, Florida. Florida Scientist 66:128-140.

Statham, M., and H. L. Statham. 1997. Movements and habits of brushtail possums (Trichosurus vulpecula Kerr) in an urban area. Wildlife Research 24:715-726.

Steen, D. A., and J. P. Gibbs. 2004. Effects of roads on the structure of freshwater turtle populations. Conservation Biology 18:1143-1148.

Taylor, B. D. and R. L. Goldingay. 2004. Wildlife road-kills on three major roads in north-eastern New South Wales. Wildlife Research 31:83-91.

Thurber, J. M., R. O. Peterson, T. D. Drummer, and S. A. Thomasma. 1994. Gray wolf response to refuge boundaries and roads in Alaska. Wildlife Society Bulletin 22:61-68.

Trimble 2003. Trimble GPS Pathfinder Office software, version 3.0. Trimble Navigation Limited, Mapping and GIS Business Area, Westminster, Colorado, USA.

Trimble 2003. Trimble TerraSync software, version 2.4. Trimble Navigation Limited, Mapping and GIS Business Area, Westminster, Colorado, USA.

Trombulak, S. C, and C. A. Frissell. 2000. Review of ecological effects of roads on terrestrial and aquatic communities. Conservation Biology 14:18-30.

Vos, C. C. 1997. Effects of road density: a case study of the moor frog. In: Canters, K. ed. Habitat fragmentation and infrastructure. 93-97. Ministry of Transportation, Public Works and Water Management, Delft, Netherlands.

Vos, C. C., and J. P. Chardon. 1998. Effects of habitat fragmentation and road density on the distribution pattern of the moor frog Rana arvalis. Journal of Applied Ecology 35:44-56.

Wake, D. B. 1991. Declining amphibian populations. Science 253:860.

Wright, A. H., and A. A. Wright. 1949. Handbook of frogs and toads of the United States and Canada. Comstock Publishing Company, Ithaca, New York, USA.

Yanes, M., J. Velasco, and F. Suarez. 1995. Permeability of roads and railways to vertebrates: the importance of culverts. Biological Conservation 71:217-222. 
Table 1. Local study sites and survey routes in Tippecanoe County, Indiana with approximate distances and site descriptions.

\begin{tabular}{|c|c|c|c|c|c|}
\hline Site & $\begin{array}{c}\text { Survey } \\
\text { Route }\end{array}$ & $\begin{array}{l}\text { Length } \\
\text { (km) }\end{array}$ & $\begin{array}{c}\text { Site } \\
\text { Description }\end{array}$ & $\begin{array}{c}\text { Road } \\
\text { Characteristics }\end{array}$ & $\begin{array}{c}\text { Urbanization } \\
\text { level }\end{array}$ \\
\hline Lindberg Rd. & $\begin{array}{l}\text { Lindberg Rd. } \\
\text { from US } 231 \\
\text { to McCormick } \\
\text { Rd. }\end{array}$ & 1.8 & $\begin{array}{l}\text { wetland } \\
\text { surrounded by } \\
\text { golf course and } \\
\text { bisected by 2-lane } \\
\text { paved road }\end{array}$ & $\begin{array}{c}\text { Straight, 2-lane } \\
\text { paved road } \\
\text { w/turning lane in } \\
\text { center; no shoulder } \\
\text { on south side, bike } \\
\text { lane on north side; } \\
\text { 30MPH }\end{array}$ & urban \\
\hline SR 26 & $\begin{array}{l}\text { SR } 26 \text { from } \\
750 \mathrm{~W} \text { to } \\
\text { CR925W }\end{array}$ & 2.9 & $\begin{array}{l}\text { wetland } \\
\text { surrounded by } \\
\text { mixed hardwood } \\
\text { woodlots and } \\
\text { agricultural fields }\end{array}$ & $\begin{array}{l}\text { Straight, semi- } \\
\text { hilly, 2-lane paved } \\
\text { road; very little } \\
\text { shoulder, some } \\
\text { roadside ditches; } \\
\text { 50-55MPH }\end{array}$ & rural \\
\hline $\begin{array}{c}\text { South River } \\
\text { Rd. }\end{array}$ & $\begin{array}{l}\text { S. River Rd. } \\
\text { from US } 231 \\
\text { bypass to } \\
\text { CR300W }\end{array}$ & 3.9 & $\begin{array}{l}\text { river bottom/flood } \\
\text { plain, mixed } \\
\text { hardwood } \\
\text { woodlots near } \\
\text { Purdue airport }\end{array}$ & $\begin{array}{c}\text { Few curves, 2-lane } \\
\text { paved road; little } \\
\text { shoulder; 40- } \\
\text { 45MPH }\end{array}$ & suburban \\
\hline US 231 & $\begin{array}{l}\text { US } 231 \text { from } \\
\text { US } 52 \text { to } \\
\text { CR600N }\end{array}$ & 3.4 & $\begin{array}{l}\text { primarily } \\
\text { agricultural with } \\
\text { semi-wet ditch } \\
\text { system }\end{array}$ & $\begin{array}{l}\text { Straight, 2-lane } \\
\text { paved road, large } \\
\text { shoulder; 55MPH }\end{array}$ & rural \\
\hline
\end{tabular}


Table 2. Remote study sites and survey routes throughout Indiana in conjunction with wildlife/aircraft collision study (fig. 2) with approximate distances and site descriptions.

\begin{tabular}{|c|c|c|c|c|c|}
\hline Site & $\begin{array}{l}\text { Survey } \\
\text { Route }\end{array}$ & $\begin{array}{l}\text { Length } \\
\text { (km) }\end{array}$ & $\begin{array}{c}\text { Site } \\
\text { Description }\end{array}$ & $\begin{array}{c}\text { Road } \\
\text { Characteristics }\end{array}$ & $\begin{array}{c}\text { Urbanization } \\
\text { level }\end{array}$ \\
\hline 1. South Bend & $\begin{array}{l}\text { SR } 4 \text { from } \\
\text { US31 to SR } \\
104\end{array}$ & 25.7 & $\begin{array}{l}\text { Potato Creek } \\
\text { SP; mixed } \\
\text { hardwoods near } \\
\text { park, mostly ag } \\
\text { fields; SR4 } \\
\text { bisects wetland }\end{array}$ & $\begin{array}{l}\text { 2-lane paved road; } \\
\text { shoulder varies; } \\
\text { 45-55MPH }\end{array}$ & suburban-rural \\
\hline 2. DeKalb & $\begin{array}{l}\text { Old State } \\
\text { Hwy } 47 \\
\text { from } 11 \mathrm{~A} \\
\text { Rd to Popp } \\
\text { Rd }\end{array}$ & 16.1 & $\begin{array}{l}\text { Primarily ag } \\
\text { fields; route } \\
\text { crosses } 3 \text { small } \\
\text { creeks }\end{array}$ & $\begin{array}{l}\text { 2-lane paved road; } \\
\text { portions of large } \\
\text { shoulder; 55MPH }\end{array}$ & rural \\
\hline 3. Warsaw & $\begin{array}{l}\text { SR } 5 \text { from } \\
\text { CR } 750 \mathrm{~N} \text { to } \\
\text { US } 33\end{array}$ & 19.3 & $\begin{array}{l}\text { Tri-County } \\
\text { FWA; route } \\
\text { adjacent to } 4 \\
\text { large lakes; } \\
\text { mostly open/ag } \\
\text { fields }\end{array}$ & $\begin{array}{l}\text { 2-lane paved road; } \\
\text { little shoulder; } 45- \\
\text { 55MPH }\end{array}$ & $\begin{array}{l}\text { urban/suburban- } \\
\text { rural }\end{array}$ \\
\hline 5. Anderson & $\begin{array}{l}\text { CR 200E } \\
\text { (old SR 67) } \\
\text { from US 36 } \\
\text { to CR 500E }\end{array}$ & 14.7 & $\begin{array}{l}\text { Mounds SP; } \\
\text { mostly open/ag } \\
\text { fields }\end{array}$ & $\begin{array}{l}\text { 2-lane paved road; } \\
\text { shoulder varies; } \\
\text { 45-55MPH }\end{array}$ & $\begin{array}{l}\text { urban-suburban- } \\
\text { rural }\end{array}$ \\
\hline 6. Richmond & $\begin{array}{l}\text { SR } 101 \text { from } \\
\text { Fosdick Rd. } \\
\text { to Golden } \\
\text { Rd. }\end{array}$ & 17.4 & $\begin{array}{l}\text { Brookville Lake } \\
\text { Project; some } \\
\text { mixed } \\
\text { hardwoods, } \\
\text { mostly open/ag } \\
\text { fields }\end{array}$ & $\begin{array}{l}\text { 2-lane paved road; } \\
\text { portions of large } \\
\text { shoulder; 55MPH }\end{array}$ & suburban-rural \\
\hline 7. Greencastle & $\begin{array}{l}\text { SR } 59 \text { from } \\
\text { CR } 720 \text { S to } \\
\text { US 36; US } \\
36 \text { from SR } \\
59 \text { to CR } \\
850 \mathrm{~W}\end{array}$ & 19.6 & $\begin{array}{l}\text { Cecil M. } \\
\text { Harden Lake } \\
\text { Project; mixed } \\
\text { hardwoods to ag } \\
\text { fields }\end{array}$ & $\begin{array}{l}\text { 2-lane paved road, } \\
\text { shoulder varies, } \\
\text { 45-55MPH }\end{array}$ & suburban-rural \\
\hline 8. Greenwood & $\begin{array}{l}\text { SR } 135 \text { from } \\
\text { SR } 44 \text { to SR } \\
\text { 144; SR } 144 \\
\text { to SR } 44\end{array}$ & 15.6 & $\begin{array}{l}\text { Mixed } \\
\text { hardwoods and } \\
\text { open/ag fields; } \\
\text { route crosses } \\
\text { several creeks }\end{array}$ & $\begin{array}{l}\text { 2-lane paved road; } \\
\text { portions of large } \\
\text { shoulder; 45- } \\
\text { 55MPH }\end{array}$ & urban-rural \\
\hline 9. Sellersburg & $\begin{array}{l}\text { SR } 160 \text { from } \\
\text { I } 65 \text { to Blue } \\
\text { River Rd }\end{array}$ & 10.5 & $\begin{array}{l}\text { Clark State } \\
\text { Forest; mixed } \\
\text { hardwoods, } \\
\text { some ag fields }\end{array}$ & $\begin{array}{l}\text { 2-lane paved road; } \\
\text { shoulder varies; } \\
\text { 45-55MPH }\end{array}$ & suburban-rural \\
\hline 10. Huntingburg & $\begin{array}{l}\text { SR } 162 \text { from } \\
\text { SR } 245 \text { to } \\
\text { US } 231 ; \text { SR } \\
62 \text { from US } \\
231 \text { to Frog } \\
\text { Pond Rd. }\end{array}$ & 19.6 & $\begin{array}{l}\text { Lincoln SP; } \\
\text { mixed } \\
\text { hardwoods, } \\
\text { some ag fields; } \\
\text { wetlands } \\
\text { bisected by } \\
\text { SR62 }\end{array}$ & $\begin{array}{l}\text { 2-lane paved road; } \\
\text { large shoulder for } \\
\text { most of route; } 45- \\
55 \mathrm{MPH}\end{array}$ & suburban-rural \\
\hline
\end{tabular}


Table 3. Habitat variables measured at each $100 \mathrm{~m} \times 200 \mathrm{~m}$ road section (modified from Malo et al. 2004).

\begin{tabular}{|l|c|}
\hline \multicolumn{1}{|c|}{ Habitat Variables } & Measure (units) \\
\hline roads & $\%$ \\
\hline grass/shrub ditch & $\%$ \\
\hline agriculture/pasture & $\%$ \\
\hline forest/woodlot & $\%$ \\
\hline urban/recreational & $\%$ \\
\hline urban/residential & $\%$ \\
\hline water/wetland & $\%$ \\
\hline grassland/shrubland & $\%$ \\
\hline \multicolumn{1}{|c|}{ Road Variables } & $\mathrm{P} / \mathrm{A}$ \\
\hline overpasses & $\mathrm{P} / \mathrm{A}$ \\
\hline underpasses & $\mathrm{P} / \mathrm{A}$ \\
\hline culverts & $\mathrm{P} / \mathrm{A}$ \\
\hline straight/curve & $\mathrm{km} / \mathrm{h}$ \\
\hline speed limit & $\mathrm{m}$ \\
\hline width & $\mathrm{number}$ \\
\hline number of lanes & \\
\hline
\end{tabular}


Table 4 . Vertebrate mortalities by taxonomic group recorded along all 13 survey routes, March 8, 2005 - July 31, 2006.

\begin{tabular}{lcccc} 
& Birds & Mammals & Herps & Total \\
\hline Lindberg Rd & 88 & 72 & 8016 & 8176 \\
SR26 & 33 & 80 & 1648 & 1761 \\
US231 & 33 & 76 & 237 & 346 \\
S. River Rd. & 51 & 132 & 49 & 232 \\
DeKalb & 5 & 117 & 11 & 133 \\
South Bend & 2 & 58 & 2 & 62 \\
Warsaw & 7 & 30 & 3 & 40 \\
Richmond & 12 & 48 & 11 & 71 \\
Anderson & 6 & 58 & 0 & 64 \\
Greenwood & 9 & 43 & 0 & 52 \\
Greencastle & 5 & 50 & 6 & 61 \\
Huntingburg & 5 & 34 & 17 & 56 \\
Sellersburg & 1 & 9 & 4 & 14 \\
Total & 257 & 807 & 10004 & $\mathbf{1 1 0 6 8}$ \\
\hline
\end{tabular}


Table 5. Vertebrate species recorded along all 4 local survey routes, March 8, 2005 - July 31, 2006.

\begin{tabular}{|c|c|c|}
\hline Scientific Name & Common Name & Total \\
\hline \multicolumn{3}{|l|}{ Mammals } \\
\hline Blarina brevicauda & northern short-tailed shrew & 14 \\
\hline Canis familiaris & domestic dog & 1 \\
\hline Canis latrans & coyote & 1 \\
\hline Didelphis virginiana & opossum & 79 \\
\hline Felis catus & cat & 5 \\
\hline Lasiurus borealis & eastern red bat & 1 \\
\hline Marmota monax & woodchuck & 1 \\
\hline Mephitis mephitis & striped skunk & 16 \\
\hline Microtus ochrogaster & prairie vole & 1 \\
\hline Microtus pennsylvanicus & meadow vole & 15 \\
\hline Mus musculus & house mouse & 2 \\
\hline Mustela vison & $\operatorname{mink}$ & 6 \\
\hline Odocoileus virginianus & white-tailed deer & 4 \\
\hline Ondatra zibethicus & muskrat & 10 \\
\hline Peromyscus spp. & deer/white-footed mouse & 39 \\
\hline Procyon lotor & raccoon & 43 \\
\hline Scalopus aquaticus & eastern mole & 4 \\
\hline Sciurus carolinensis & eastern gray squirrel & 23 \\
\hline Sciurus niger & eastern fox squirrel & 27 \\
\hline Sorex cinereus & masked shrew & 1 \\
\hline Spermophilus tridecemlineatus & 13-lined ground squirrel & 6 \\
\hline Sylvilagus floridanus & eastern cottontail & 37 \\
\hline Tamiasciurus hudsonicus & red squirrel & 6 \\
\hline Tamias striatus & eastern chipmunk & 7 \\
\hline \multirow[t]{4}{*}{ Vulpes vulpes } & red fox & 1 \\
\hline & unknown bat & 2 \\
\hline & unknown mammal & 8 \\
\hline & Total & 360 \\
\hline
\end{tabular}

\section{Birds}


Branta canadensis

Butorides virescens

Cardeulis tristis

Cardinalis cardinalis

Chaetura pelagica

Colaptes auratus

Dumetella carolinensis

Eremophila alpestris

Hirundo rustica

Melanerpes erythrocephalus

Melospiza melodia

Molothrus ater

Otus asio

Passer domesticus

Passerina cyanea

Phasianus colchicus

Porzana carolina

Quiscalus quiscula

Spizella passerina

Sturnella magna

Sturnus vulgaris

Tachycineta bicolor

Troglodytes aedon

Turdus migratorius

Zenaida macroura
Canada goose 2

green heron 1

American goldfinch 1

northern cardinal 9

chimney swift $\quad 36$

northern flicker 1

gray catbird 1

horned lark 1

barn swallow 5

red-headed woodpecker 2

song sparrow 9

brown-headed cowbird 2

eastern screech owl 6

house sparrow $\quad 15$

indigo bunting 3

ring-necked pheasant 2

Sora 1

common grackle 6

chipping sparrow 1

eastern meadowlark 2

European starling 11

tree swallow 1

house wren 1

American robin 18

mourning dove 4

unknown bird $\quad 56$

Total 205

\section{Herps}

Ambystoma tigrinum

eastern tiger salamander

142

American toad 111

snapping turtle 23

midland painted turtle 28

black rat snake 5

fox snake 9 
Graptemys geographica

Hyla spp.

Nerodia sipedon

Pseudacris crucifer

Rana catesbeiana

Rana clamitans

Rana palustris

Rana pipiens

Rana spp.

Storeria dekayi wrightorum

Terrapene carolina

Thamnophis sirtalis

Trachemys scripta northern map turtle

tree frog

northern water snake

spring peeper

bullfrog

green frog

pickerel frog

northern leopard frog

unknown ranid

midland brown snake

eastern box turtle

garter snake

red-eared slider

unknown frog

unknown snake

unknown turtle

Total

Overall total
10515 
Table 6. Vertebrate species recorded along all 13 survey routes, March 8, 2005 - July 31, 2006.

\begin{tabular}{llr}
\hline \multicolumn{1}{c}{ Scientific Name } & Common Name & Total \\
\hline Mammals & northern short-tailed shrew & 14 \\
Blarina brevicauda & domestic dog & 3 \\
Canis familiaris & coyote & 3 \\
Canis latrans & opossum & 258 \\
Didelphis virginiana & cat & 25 \\
Felis catus & eastern red bat & 2 \\
Lasiurus borealis & woodchuck & 5 \\
Marmota monax & striped skunk & 34 \\
Mephitis mephitis & prairie vole & 1 \\
Microtus ochrogaster & meadow vole & 16 \\
Microtus pennsylvanicus & house mouse & 2 \\
Mus musculus & mink & 10 \\
Mustela vison & white-tailed deer & 26 \\
Odocoileus virginianus & muskrat & 12 \\
Ondatra zibethicus & deer/white-footed mouse & 45 \\
Peromyscus spp. & raccoon & 177 \\
Procyon lotor & Norway rat & 1 \\
Rattus norvegicus & eastern mole & 5 \\
Scalopus aquaticus & eastern gray squirrel & 26 \\
Sciurus carolinensis & eastern fox squirrel & 42 \\
Sciurus niger & masked shrew & 1 \\
Sorex cinereus & 13-lined ground squirrel & 7 \\
Spermophilus tridecemlineatus & eastern cottontail & 60 \\
Sylvilagus floridanus & red squirrel & 6 \\
Tamiasciurus hudsonicus & eastern chipmunk & 7 \\
Tamias striatus & red fox & 2 \\
Vulpes vulpes & unknown bat & 2 \\
& unknown mammal & 15 \\
& Total & 807
\end{tabular}

\section{Birds}

Accipiter cooperii

Agelaius phoeniceus

Anas platyrhynchos

Branta canadensis

Butorides virescens

Cardeulis tristis

Cardinalis cardinalis

Chaetura pelagica

Colaptes auratus

Dumetella carolinensis

Coooper's hawk 1

red-winged blackbird 9

mallard 1

Canada goose 2

green heron 1

American goldfinch 3

northern cardinal $\quad 12$

chimney swift 37

northern flicker 1

gray catbird 1 
Eremophila alpestris

Falco sparverius

Hirundo rustica

Junco hyemalis

Melanerpes erythrocephalus

Meleagris gallopavo

Melospiza melodia

Mimus polyglottos

Molothrus ater

Otus asio

Passer domesticus

Passerina cyanea

Phasianus colchicus

Poecile atricapillus

Porzana carolina

Quiscalus quiscula

Spizella passerina

Sturnus vulgaris

Tachycineta bicolor

Troglodytes aedon

Turdus migratorius

Tyrannus tyrannus

Zenaida macroura
Sturnella magna

horned lark

American kestrel

barn swallow

dark-eyed junco

red-headed woodpecker

wild turkey

song sparrow

northern mockingbird

brown-headed cowbird

eastern screech owl

house sparrow

indigo bunting

ring-necked pheasant

black-capped chickadee

Sora

common grackle

chipping sparrow

eastern meadowlark

European starling

tree swallow

house wren

American robin

eastern kingbird

mourning dove

unknown bird

unknown swallow

unknown sparrow

Total

257

\section{Herps}

Ambystoma tigrinum

Bufo americanus

Chelydra serpentina

Chrysemys picta

Coluber constrictor constrictor

Coluber constrictor foxii

Elaphe obsoleta

Elaphe vulpina

Graptemys geographica

Hyla spp.

Lampropeltis getula nigra

Lampropeltis triangulum

Pseudacris crucifer

Rana catesbeiana

Rana clamitans

Rana palustris eastern tiger salamander

American toad

112

snapping turtle

25

midland painted turtle

40

northern black racer

1

blue racer

black rat snake

fox snake

northern map turtle

tree frog

black kingsnake

eastern milk snake

spring peeper

bullfrog

green frog

pickerel frog

.

. 
Rana pipiens

Rana spp.

Storeria dekayi wrightorum

Terrapene carolina

Thamnophis sirtalis

Trachemys scripta northern leopard frog

75

unknown ranid

7605

midland brown snake

19

eastern box turtle

10

garter snake

41

red-eared slider

17

unknown frog

16

unknown snake

unknown turtle

Total

4

10004

Overall total

11068 
Table 7. Habitat variables used to predict roadkill numbers along each local route per $100 \mathrm{~m}$ section.

\begin{tabular}{|c|c|c|c|}
\hline Ste & Variable & Model R-Square & \\
\hline
\end{tabular}

\begin{tabular}{llllll}
1 & urbrecresid & 0.9665 & 1367.38 & 461.83 & $<.0001 *$ \\
2 & waterresid & 0.9868 & 534.560 & 22.91 & $0.0002 *$ \\
3 & urbresresid & 0.9947 & 208.709 & 20.99 & $0.0004 *$ \\
4 & forresid & 0.9982 & 66.0821 & 25.38 & $0.0002 *$ \\
5 & roads & 0.9996 & 9.1780 & 46.57 & $<.0001 *$ \\
6 & ag & 0.9997 & 7.0000 & 4.18 & 0.0656 \\
\hline
\end{tabular}

\section{US 231}

\begin{tabular}{llllll}
1 & urbres & 0.1691 & 1.8004 & 6.71 & $0.0141 *$ \\
2 & water & 0.0547 & 1.6420 & 2.25 & 0.1431 \\
3 & roads & 0.0516 & 1.6041 & 2.21 & 0.1473 \\
\hline
\end{tabular}

South River Rd.

\begin{tabular}{llllll}
1 & ditch & 0.1115 & 2.4937 & 4.9 & $0.0328 *$ \\
2 & roads & 0.1761 & 1.6245 & 2.98 & 0.0926 \\
\hline & & SR 26 & & & \\
& & & & & \\
1 & water & 0.7577 & 7.6290 & 87.57 & $<.0001 *$ \\
2 & forest & 0.0344 & 4.8572 & 4.46 & $0.044 *$ \\
\hline
\end{tabular}

* significant at $\alpha 0.05$ 
Figure 1. Map showing locations of local survey routes $(n=4)$.

Figure 2. Map showing locations of remote survey routes in conjunction with wildlife/aircraft study $(n=9)$.

Figure 3. Lindberg Rd. survey route with digitized habitat types and 100m buffer.

Figure 4. South River Rd. survey route with digitized habitat types and 100m buffer.

Figure 5. SR 26 survey route with digitized habitat types and $100 \mathrm{~m}$ buffer.

Figure 6. US 231 survey route with digitized habitat types and $100 \mathrm{~m}$ buffer.

Figure 7. March 2005 through July 2006 monthly roadkill levels vs. mean temperature and precipitation across all local routes.

Figure 8. March 2005 through July 2006 monthly roadkill levels vs. mean temperature and precipitation on Lindberg Rd. route.

Figure 9. March 2005 through July 2006 monthly roadkill levels vs. mean temperature and precipitation on US 231 route

Figure 10. March 2005 through July 2006 monthly roadkill levels vs. mean temperature and precipitation on South River $\mathrm{Rd}$. route

Figure 11. March 2005 through July 2006 monthly roadkill levels vs. mean temperature and precipitation on SR 26 route.

Figure 12. Canada geese along Lindberg Rd (photo courtesy of R. Zeiber).

Figure 13. Chimney swift on shoulder of Lindberg Rd (photo courtesy of R. Zeiber).

Figure 14. Mass of roadkilled anurans discovered during Lindberg Rd. survey.

Figure 15. Subadult bullfrog mortality on Lindberg Rd. 


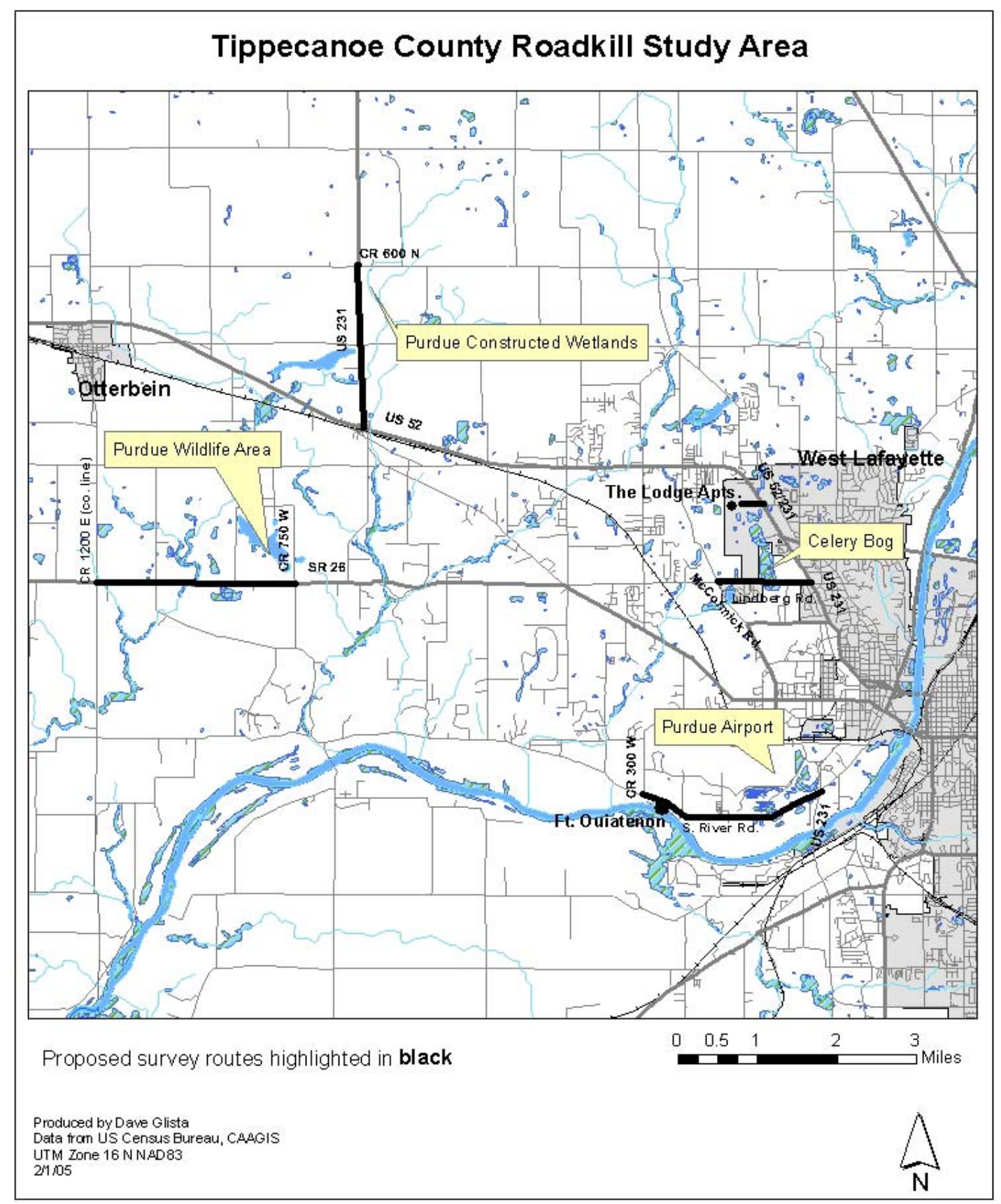




\section{Indiana Airports Participating in Wildlife-Aircraft Collision Study}

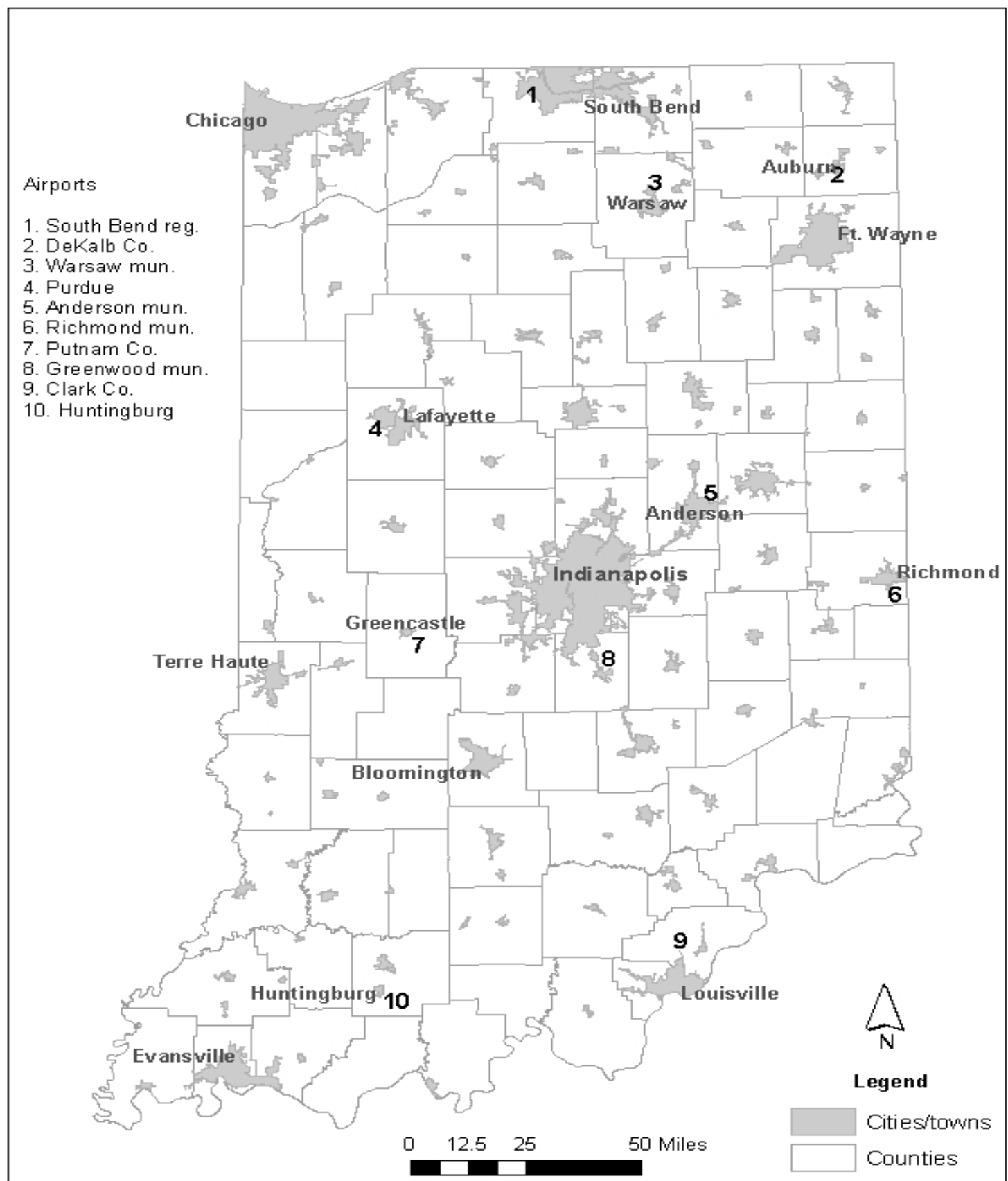

Produced by Dave Glista

Data from CAAGIS

UTM Zone 16 N NAD83

21,05 


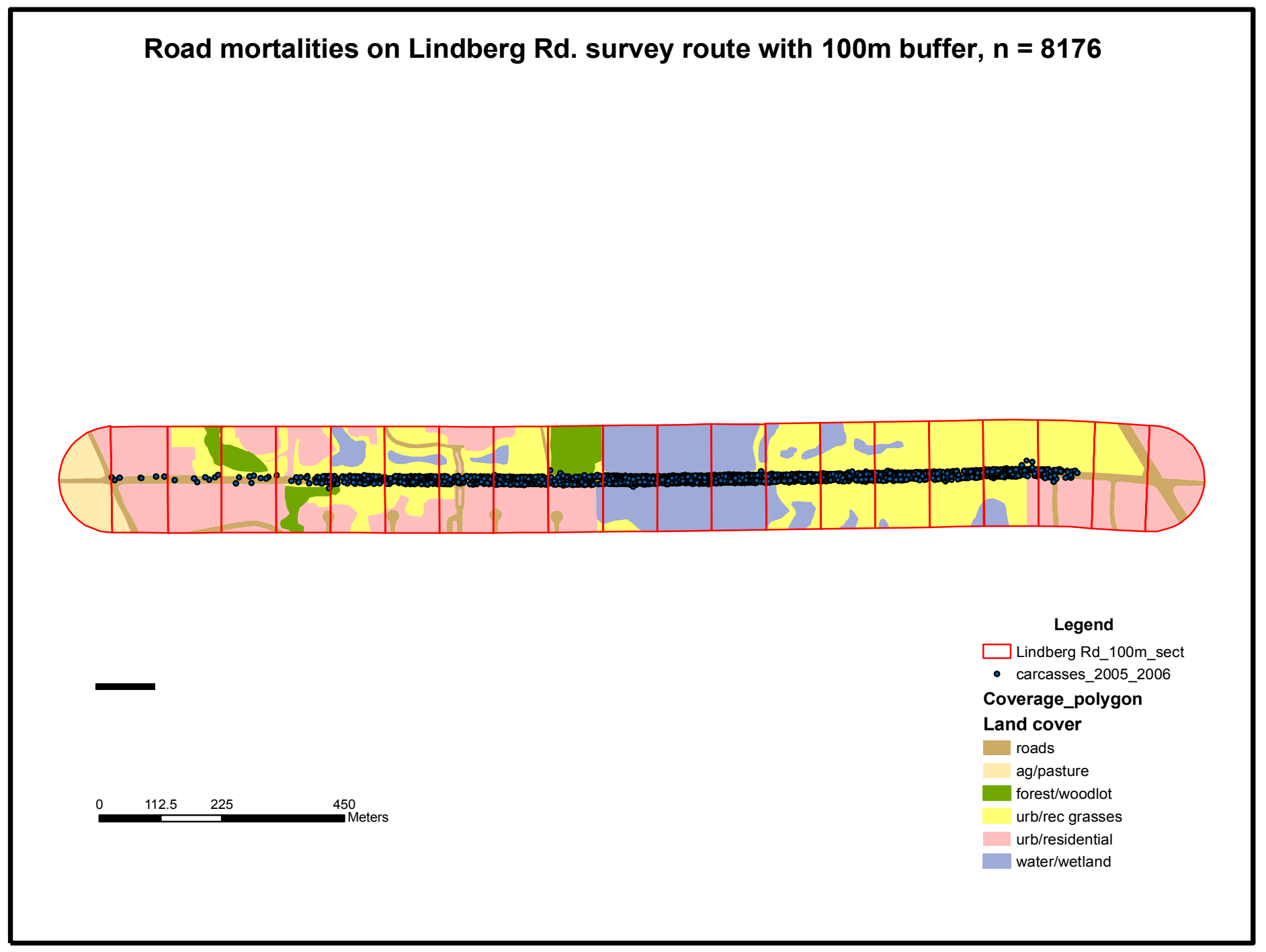


Road mortalities on South River Rd. survey route with $100 \mathrm{~m}$ buffer, $\mathrm{n}=\mathbf{2 3 2}$

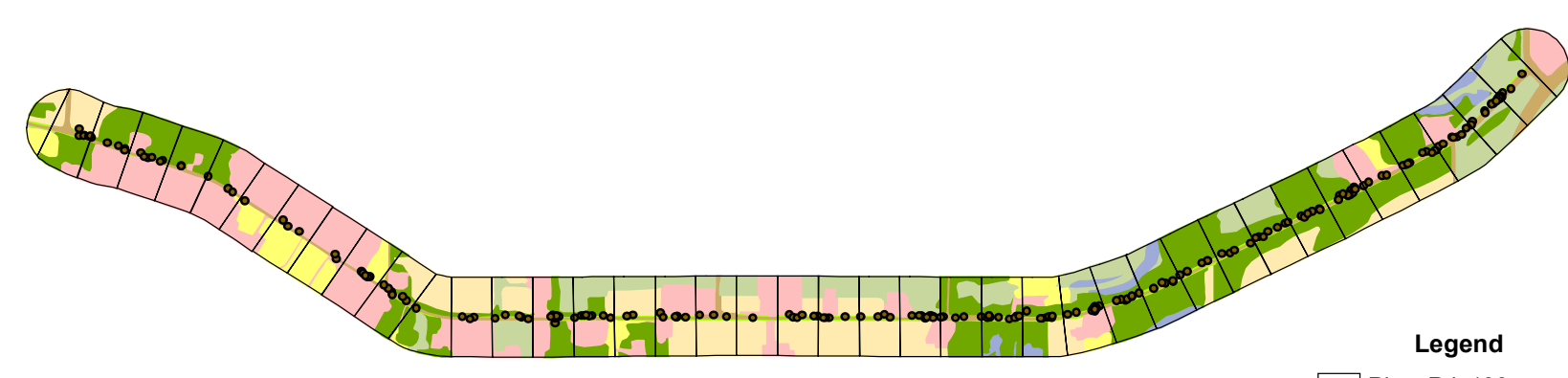

$\square$ River Rd_100m sect

- carcasses_2005_2006

Coverage polygon

Land cover

roads

grass/shrib ditch

ag/pasture

forest/woodlot

urb/rec grasses

urb/residential

water/wetland

grass/shrubland 


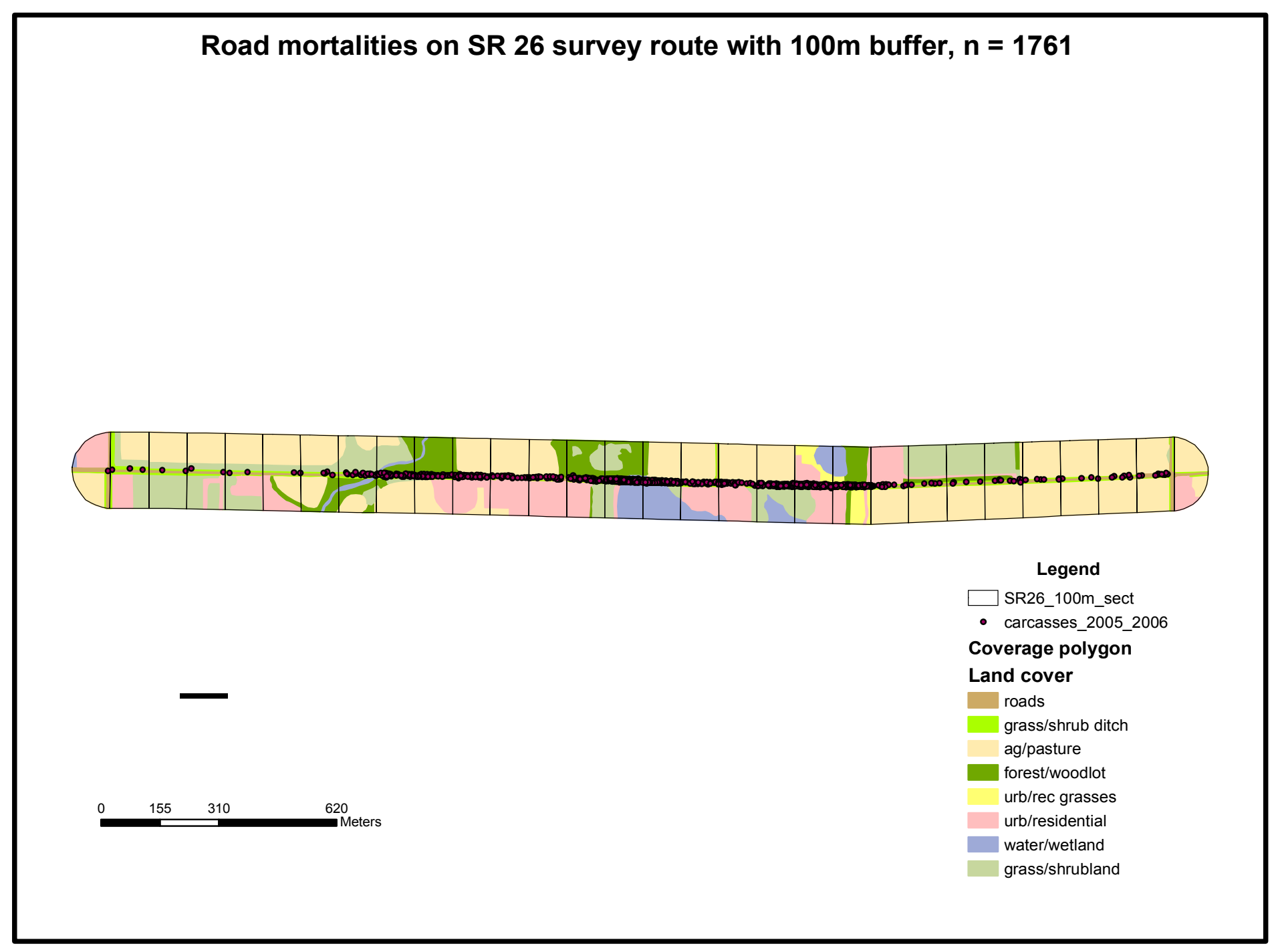




\section{Road mortalities on US 231 survey route with $100 \mathrm{~m}$ buffer, $\mathrm{n}=346$}

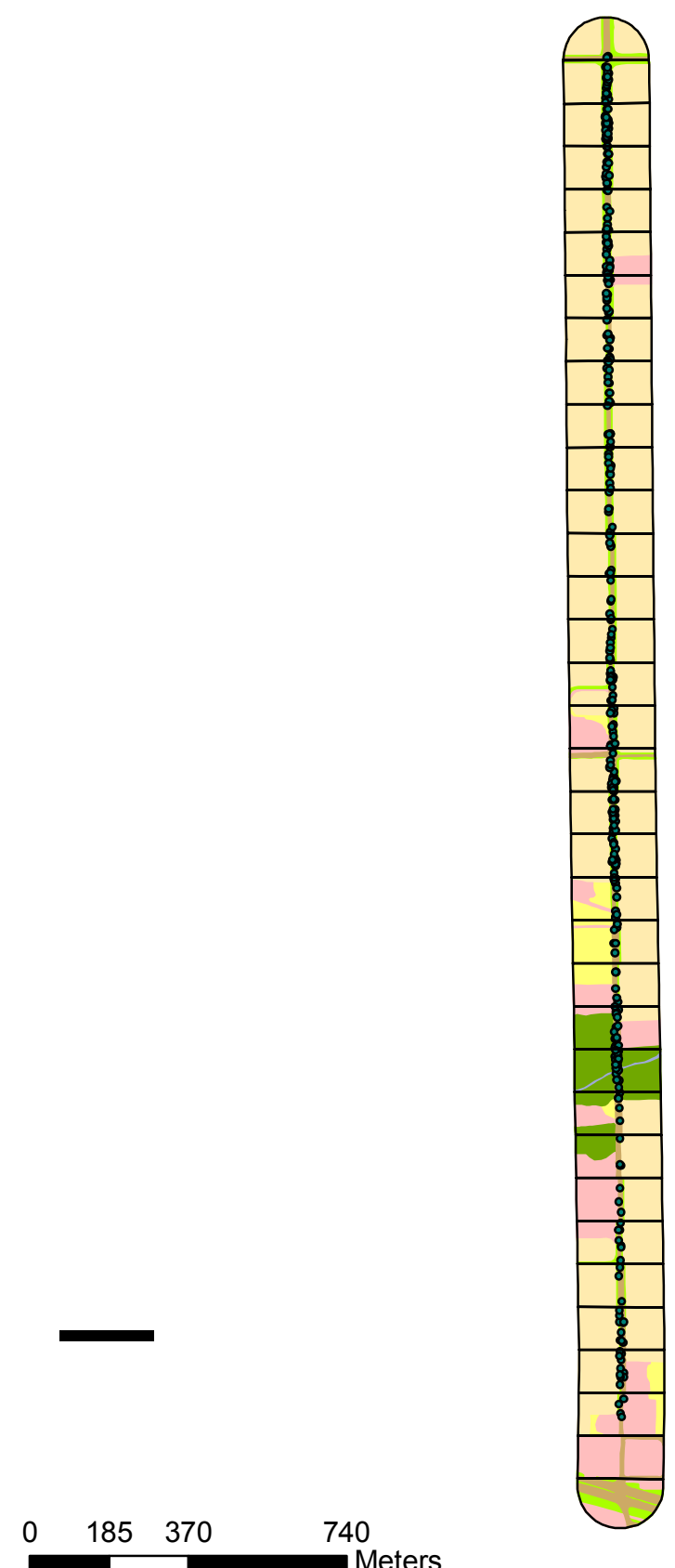

\section{Legend}

US231_100m_sect

- carcasses_2005_2006

Coverage polygon Land cover

roads

grass/shrub ditch ag/pasture

forest/woodlot

urb/rec grasses

urb/residential

water/wetland 


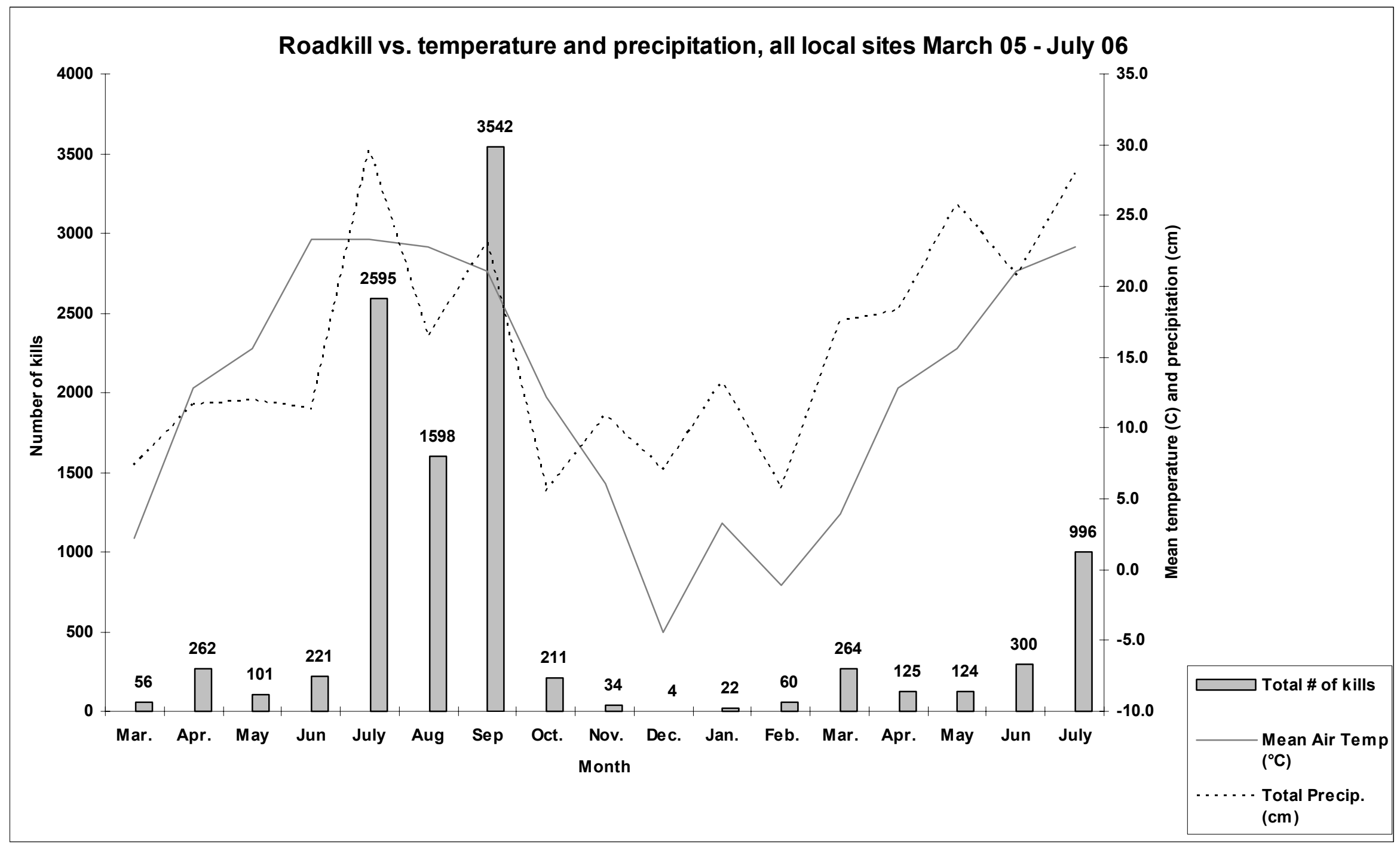




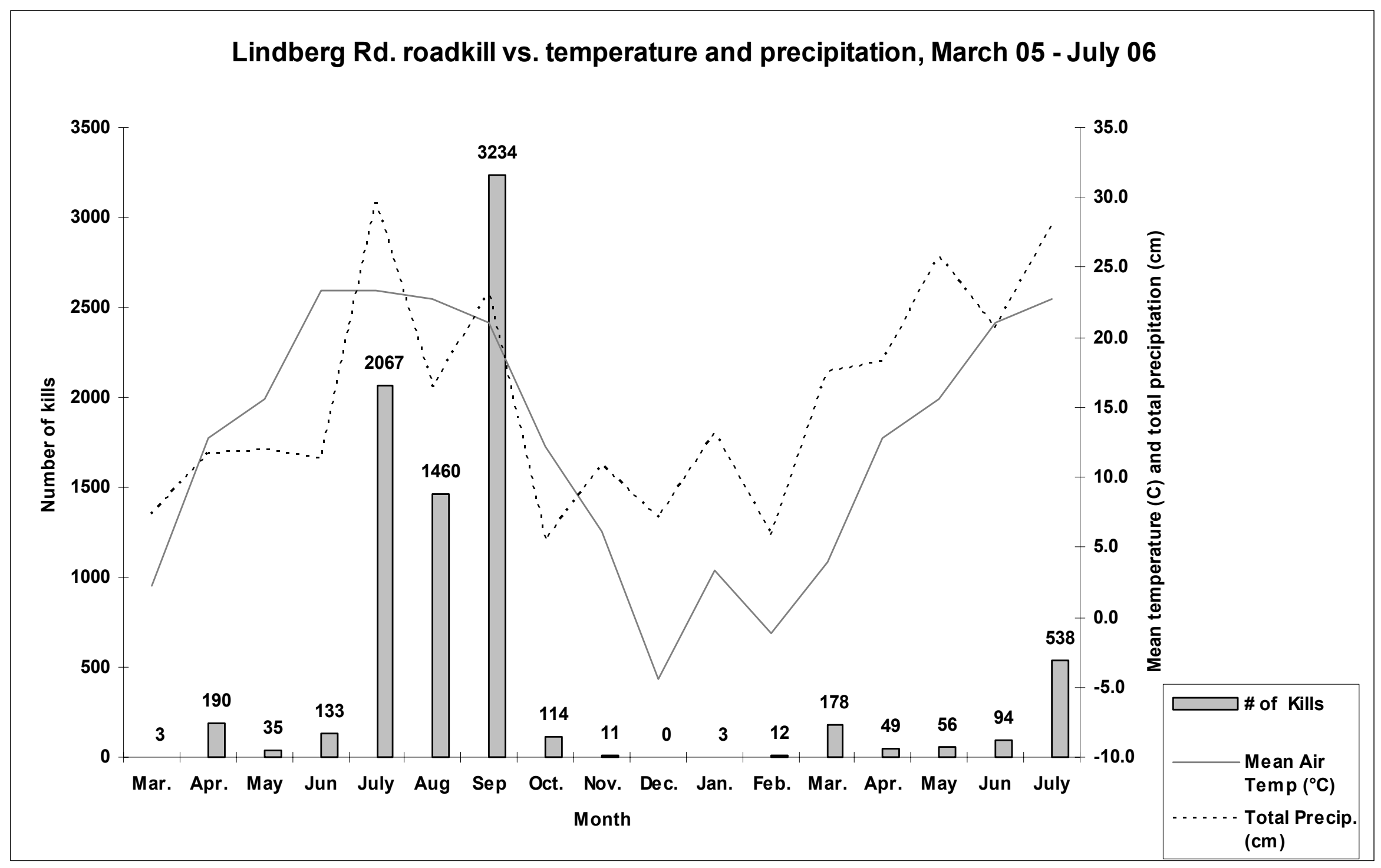




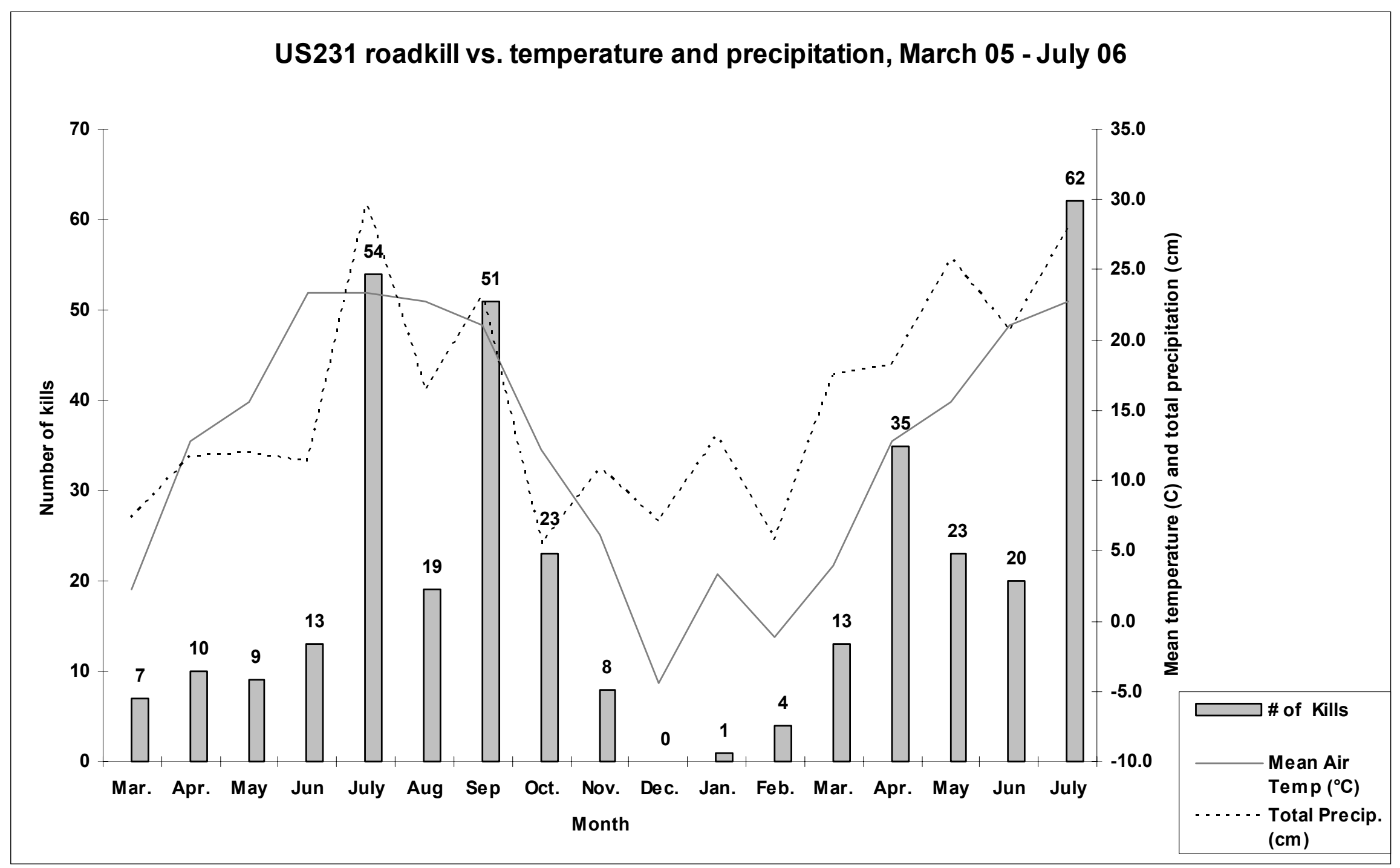




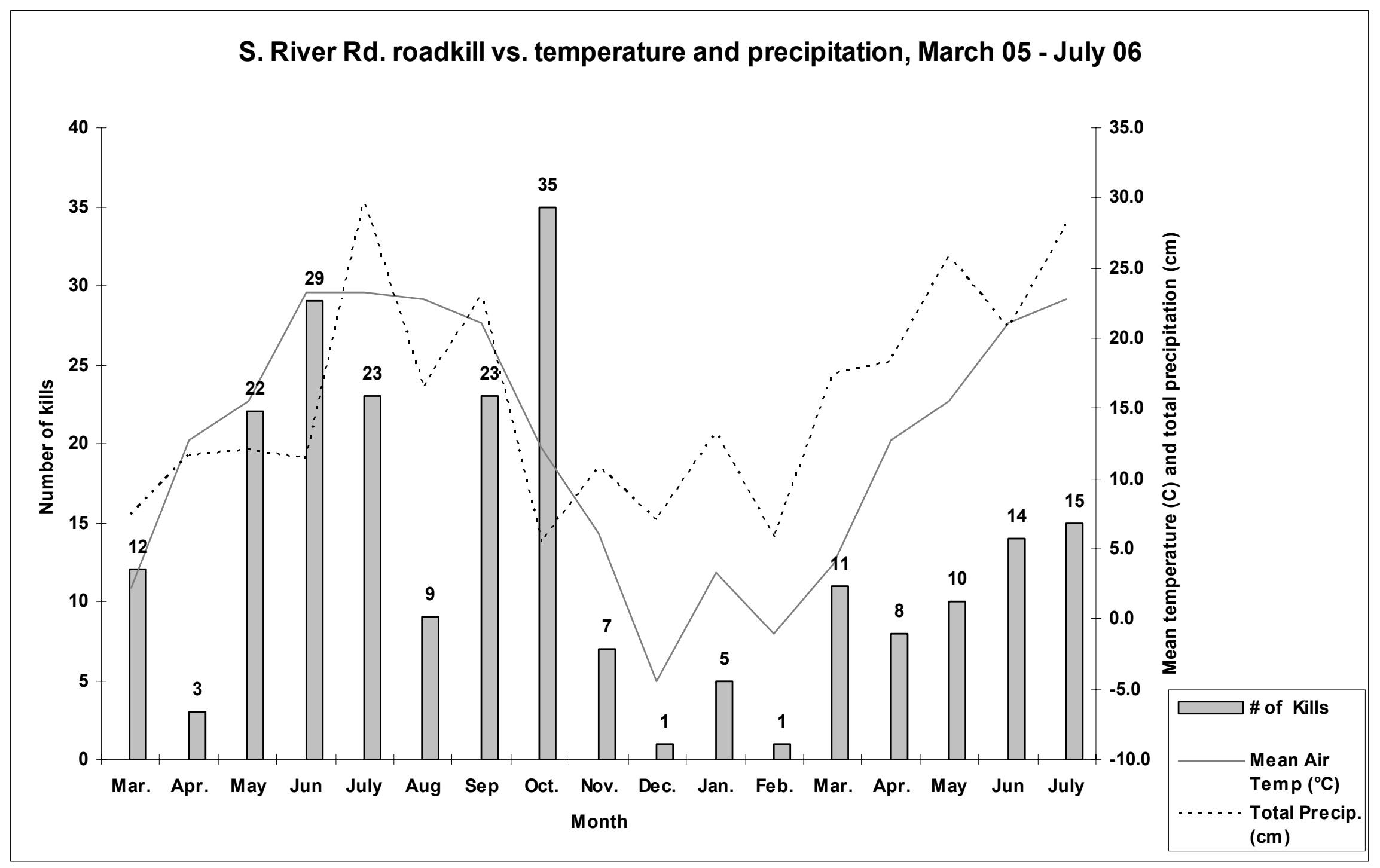




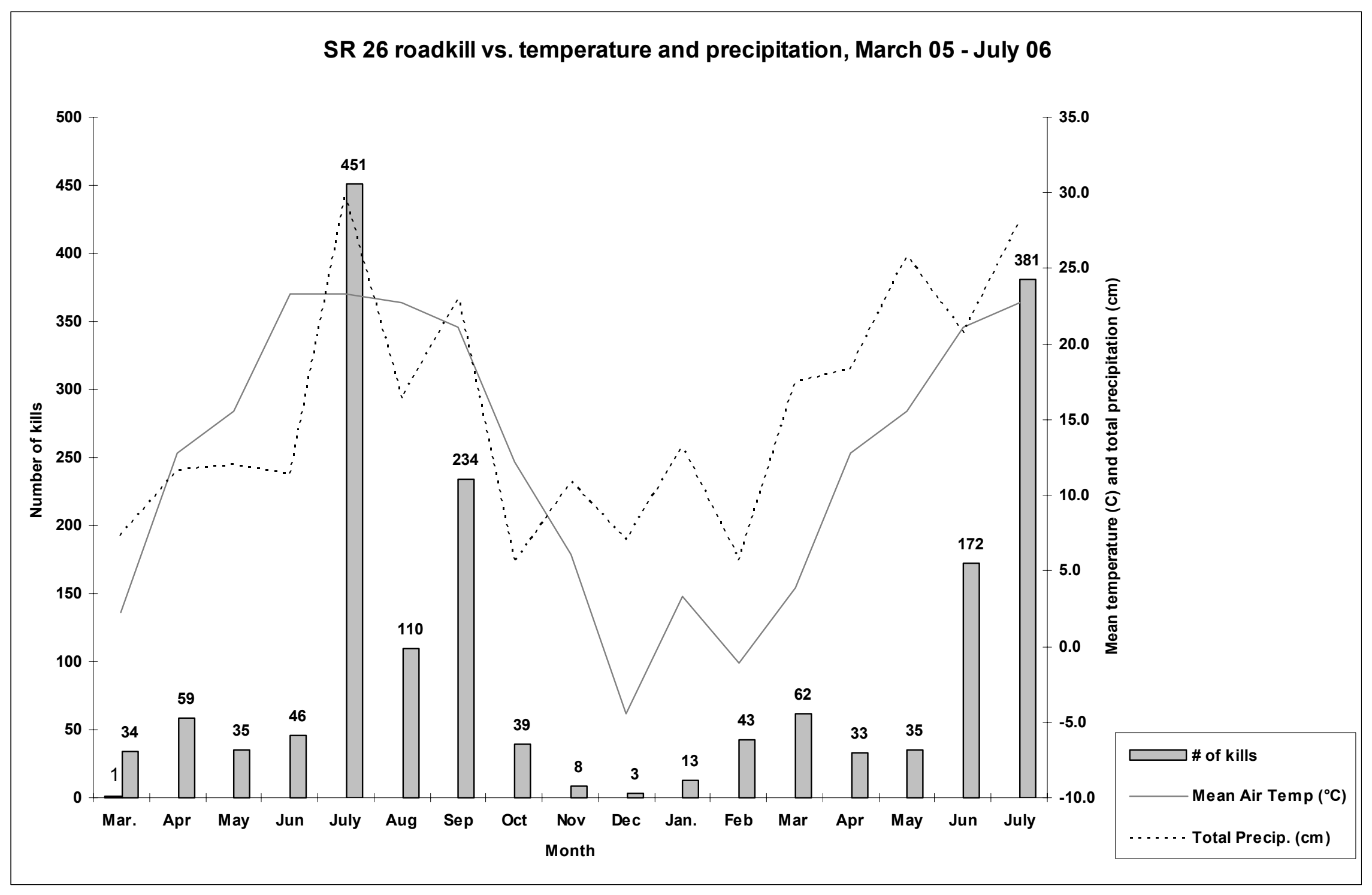



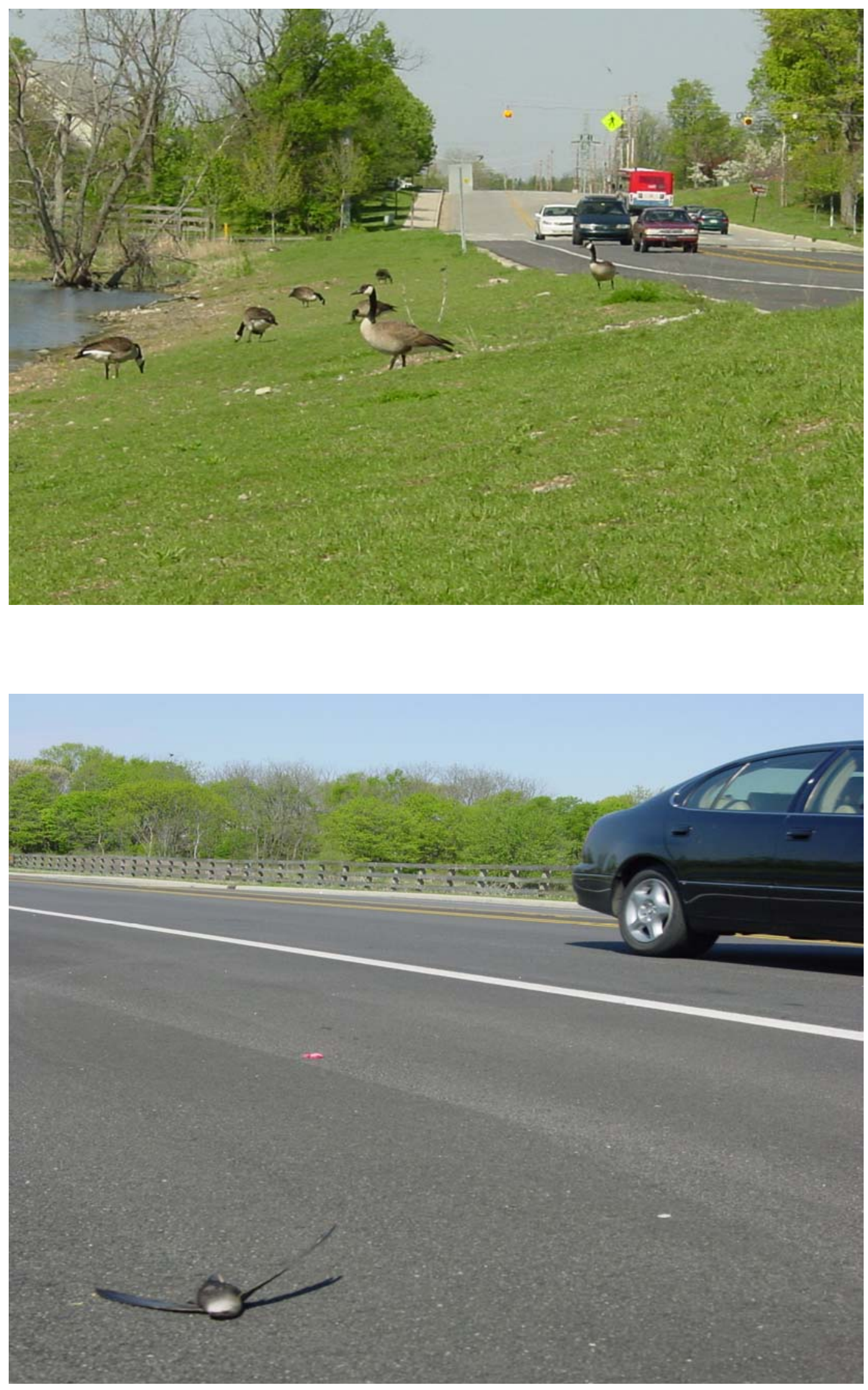

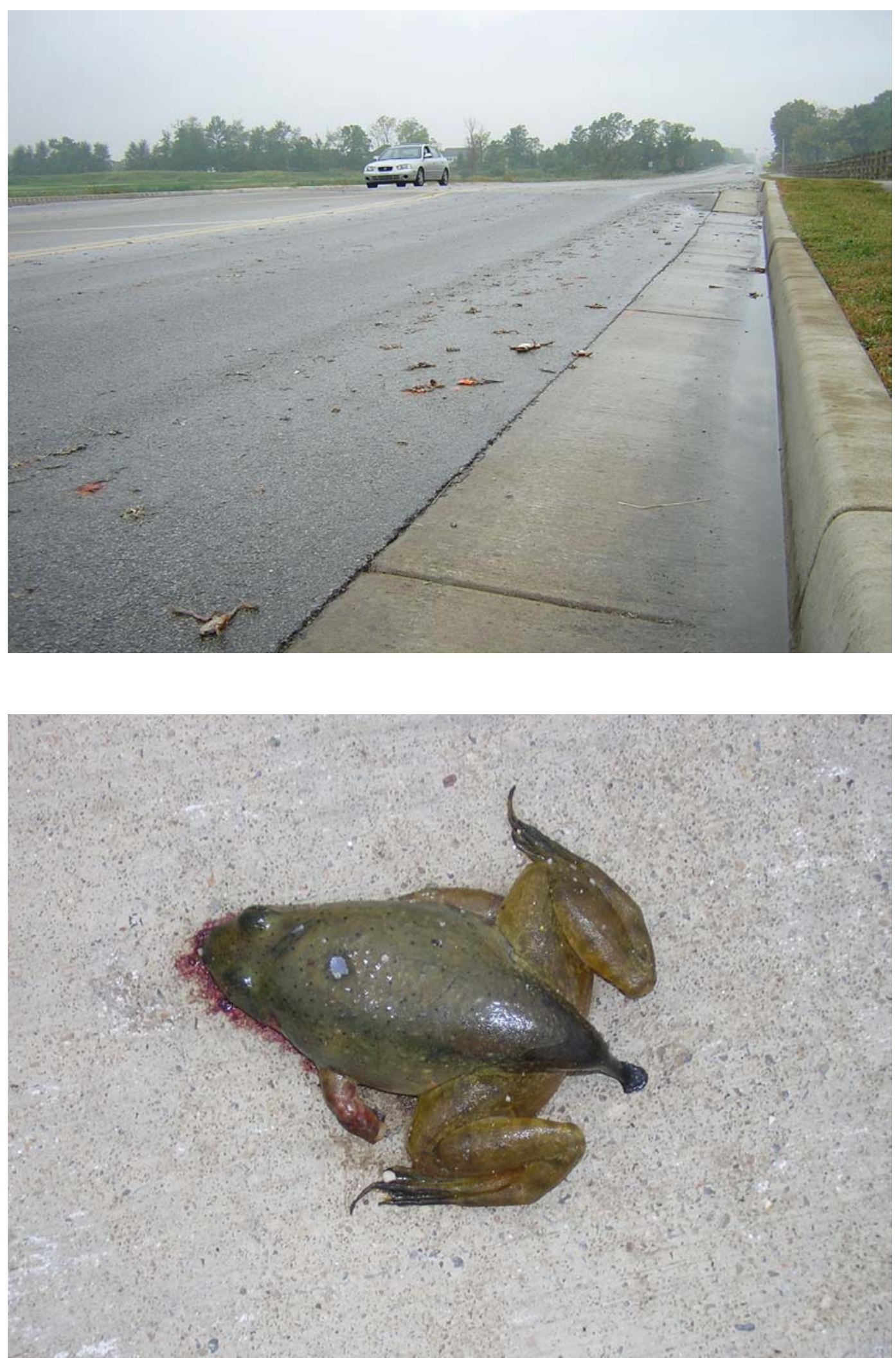


\section{CHAPTER 2. MITIGATION MEASURES FOR REDUCING WILDLIFE MORTALITY ON ROADWAYS: A REVIEW}

\section{EXECUTIVE SUMMARY}

There are over 6 million $\mathrm{km}$ of roads throughout the United States linking metropolitan areas and providing convenient routes of travel for both the commercial and private sectors. While this road system is important to the infrastructure of the country it can also pose a threat to various wildlife species in the form of both physical and psychological barriers and wildlife/vehicle collisions. Collisions with wildlife can be a major source of animal mortality and can be expensive and dangerous for motorists. Europe has led the way in the development of wildlife road mortality mitigation measures but the issue of road mortality mitigation in North America is becoming increasingly prudent.

There are several types of measures that can be implemented to reduce the effects of roads on a variety of wildlife species. Pipe and box culverts can often be installed to facilitate the crossing of roads by numerous smaller animals. Underpasses are usually much larger in scale than culverts and incorporate the natural habitat in the area, such as large stream crossings. Wildlife overpasses, also referred to as land bridges are designed to allow wildlife to cross over a road and are much more open in design. Traffic control and driver behavior modification can also be effective at times. Various nonstructural methods such as repellents that work against animal senses and habitat modifications are currently being researched, which may lead to innovative and less expensive alternatives to some of the more costly constructed measures. Several biological factors need to be considered (e.g. species of concern, habitat requirements, life history, etc.) before 
deciding on which mitigation measures may be most appropriate. Furthermore, any implemented mitigation measures must be monitored post-construction to determine their true effectiveness.

\section{INTRODUCTION}

Roads are an important part of the infrastructure of the world. Roads allow people and goods to move from place to place in a relatively expeditious manner. In the United States, the 6.2 million km system of public roads, used by 200 million vehicles, links essentially every local area (National Research Council 1997).

While roads are an important part of the infrastructure and can provide some ecological benefits such as maintenance of grassland plants in intense agricultural areas (Forman 2000), they can also present several ecological problems such as acting as both physical and biological barriers to many wildlife species (Jackson 2000, Forman and Alexander 1998). Roads can affect the quality and quantity of available wildlife habitat, most notably through fragmentation. Likewise, roads can be direct sources of wildlife mortality and in some instances, act as a predatory mechanism (Langton 1989). Many other ecological effects of roads on species, soils, and water have been identified, with effects varying in distance outward from meters to kilometers (Ellenberg et al. 1991, Forman 1995). These "road-effect zones" impact an estimated 15-20\% of the United States (Forman and Alexander 1998).

Collisions with automotive traffic can be a major source of mortality in animal populations (Romin and Bissonette 1996, Trombulak and Frissell 2000, Gibbs and Shriver 2002). Lalo (1987) estimated vertebrate mortality on United States roads at 1 million individuals per day. There are a variety of mitigation approaches that can be used 
to reduce the effects of roads and road mortality on wildlife populations. In general, these approaches fall under one of two umbrellas: the modification of motorist behavior and/or the modification of animal behavior. Modifying motorist behavior often involves speed limits, lights, and signs whereas animal behavior can be modified by habitat alterations and wildlife crossing structures (Romin and Bissonette 1996, Forman et al. 2003). Wildlife crossing structures range from exclusion fences and culverts to overpass/underpass systems (Romin and Bissonette 1996, see Appendix). Many structures are designed to reduce large animal-vehicle collisions (Forman et al. 2003). Such structures must be designed to allow safe passage for animals, promote habitat connectivity, be accessible, and encourage natural movement. Unfortunately, the measures often used by states do not correlate with the perceived success of the measures and the most promising measures are the least used (Forman et al. 2003). This is unfortunate, because poor designs do little to minimize road effects and are generally a waste of time and money (Forman et al. 2003). Moreover they can interrupt natural processes which can lead to various ecological problems such as overgrazing, increased erosion, or population declines (Forman et al. 2003).

A growing literature in the field of road ecology suggests that vehicle/wildlife collisions are important to biologists and transportation officials alike. In this review, we summarized previous wildlife mitigation monitoring studies (Table 1), described some of the most common mitigation measures employed, and discussed factors that lend to their overall effectiveness. 


\section{TYPES OF CROSSING STRUCTURES}

\section{Pipe and Box Culverts}

Pipe culverts are relatively small structures (1-6ft. diameter) made of concrete, smooth steel, or corrugated metal designed to carry water under roads (Figure 1). Europe has led the way in implementing smaller pipe-style culverts, also referred to as "amphibian tunnels" (Forman et al. 2003, Figures 1 and 2). Box culverts, generally larger than pipe culverts, are also used to allow water to pass under roads (Figures 3 and 4). Unlike pipe culverts they usually remain dry except in periods of heavy run off. Culverts may be used by a variety of wildlife species (Rodriguez et al. 1996, Yanes et al. 1995, Clevenger and Waltho 2000). Kaye et al. (2005) reported that the use of a box culvert under a highway improvement project in Massachusetts allowed the crossing of spotted turtles (Clemmys guttata, a state threatened species) between two turtle habitats. The use of a culvert system reduced roadkill numbers in the Paynes Prairie State Preserve, Florida, by 93.5\% (Dodd 2004). Clevenger et al. (2001) monitored 36 culverts along the Trans-Canada highway and found a total of 618 crossings by a minimum of 9 species, with an average of 2.8 species at each culvert. In Australia, Taylor and Goldingay (2003) recorded 17 different vertebrate species using purpose-built fauna culverts in combination with exclusion fence under the Pacific Highway.

\section{Underpasses}

Wildlife underpasses, also known as wildlife bridges, are large underpasses that provide a relatively unconfined passage for wildlife (Jackson and Griffin 2000). Where roads cross over water or other roads, bridges can provide a passageway for many wildlife species, especially those that use riparian corridors (Figure 5). In situations 
where underpasses may hold excessive amounts of water, ledges can be incorporated into the designs to allow animal passage (Figure 6). Veenbaas and Brandjes (1999) carried out a survey in 1997 to investigate the use of faunal passages in existing highway underpasses along waterways and found that mammals were present at all fauna passages and $75 \%$ of passages were used by amphibians. Underpasses with the largest diameters were used most frequently by mammals; this relationship did not hold for amphibians. Passages with extended banks were used by more species overall.

\section{Overpasses}

Overpasses for wildlife are mainly designed for larger animals such as large carnivores and ungulates (Figure 7). They can range from 30-50m in width (perpendicular to the road) and span over 200m (Jackson and Griffin 2000, Forman et al. 2003). Overpasses are sometimes referred to as green bridges. Green bridge is a term used to refer to a wildlife overpass with a relatively large strip of natural vegetation crossing over a road (Bekker et al. 1995). Landscape connectors are especially wide overpasses that maintain the connectivity of horizontal ecological flows across the landscape (Forman et al. 1997). Wildlife overpasses seem to accommodate a larger variety of species than underpasses (Jackson and Griffin 2000). Some advantages to overpasses are that they are less confining, quieter, maintain ambient conditions of rainfall, temperature, and light, and can serve as both passageways for wildlife and intermediate habitat for smaller animals (e.g. small mammals, reptiles, and amphibians) (Jackson and Griffin 2000). One of the drawbacks to overpasses is that they are often the most expensive option due to construction costs. 
Van Wieren and Worm (2001) evaluated a wildlife overpass in the central Netherlands and found that the most frequent users of the pass were large mammals, specifically red deer (Cervus elaphus) and wild boar (Sus scrofa). They also noted that crossings had increased almost threefold since previous monitoring in 1989 and suggested that the increase was due to habituation of red deer to the structure. Keller (1999) also noted that ungulates, most notably roe deer (Capreolus capreolus) were the most frequent users of wildlife overpasses in Switzerland, Germany, France, and the Netherlands. Clevenger and Waltho (2005) also found that larger mammals frequented overpasses in Banff National Park, Canada. They monitored 2 overpass structures along the Trans-Canada highway and found that elk (Cervus elaphus) and deer (Odocoileus spp.) were the most frequent large mammals to use the structures.

\section{NONSTRUCTURAL METHODS}

Cost is often a major concern when discussing which wildlife road mortality mitigation measures to implement. Costs can be extremely variable depending on the method chosen, availability of materials, and scale of the project. Structural methods will almost always be more expensive than nonstructural methods. While nonstructural methods may be less expensive, they may still prove effective—-but like all mitigation methods, they need to be researched and their effectiveness evaluated. Bank et al. (2002) reported on a variety of nonstructural methods currently being researched in Europe. These include 1) olfactory repellents in which scented foam is sprayed on vegetation and structures along the road, 2) ultrasound, 3) road lighting (which may have unfortunate negative consequences for nesting birds), 4) population control (e.g., hunting), which is most effective for local populations, and 5) habitat modification, used primarily to keep 
animals away from the road or increase to increase driver and animal visibility. If cheaper alternatives to expensive structures (e.g., overpasses) are found to be effective at mitigating roadkill, it would allow wider use and still promote permeability across roads (Forman et al. 2003).

\section{Traffic Control}

While it is difficult to predict exactly where and when animals will appear on roads, making motorists aware of the potential for animals crossing can sometimes help mitigate wildlife road mortality. One way to accomplish this is by reducing vehicle speed in areas of known animal crossings through the use of signs and/or speed bumps and enhancing speed limit enforcement. High-speed traffic is often considered one of the main causes of wildlife-vehicle collisions (Pojar et al. 1975, Case 1978). Wildlife crossing signs can also be installed in areas of known animal crossings to help make drivers more aware of wildlife presence. Unfortunately, signs may be relative ineffective (Pojar et al. 1975). Aberg (1981, as cited in Groot Bruinderink and Hazebroek 1996) studied the effectiveness of wildlife-crossing signs and the ability of drivers to detect moose decoys with only $40 \%$ noticing the signs and/or decoys. Even stuffed mule deer (Odocoileus hemionus) placed in road rights-of-way failed to evoke a reaction from many

drivers (D. F. Reed, personal communication as cited in Groot Bruinderink and Hazebroek 1996).

\section{MITIGATION FOR BIRDS}

While most wildlife road mortality mitigation measures center on mammals, reptiles, and amphibians it must be mentioned that roads also can also affect birds (e.g., fragmentation, isolation, and direct mortality; Table 2). One advantage that most birds 
have over other taxa is the ability to fly over roads rather than walk or run across them, thus allowing them a safer mode of travel from point A to point B. However, birds have some unique problems. Birds often define territories by use of songs and if those songs cannot be heard over (or are distorted by) vehicular traffic noise, males may find it difficult to attract and keep mates (Ferris 1979, Reijnen and Foppen 1995). This could potentially force males to conduct wider searches for females and bring them into closer proximity to roads. Many migrating species rely on starlight navigation (Emlen 1975) and light pollution from a variety of sources, including highway lighting, may cause birds to become disoriented and result in collisions with automobiles (Ogdon 1996). Non- or low-flying birds (e.g., quail, turkey, owls), birds that forage at ground level, and scavengers are even more susceptible to road mortality because of their habits (Stoner 1925). Jacobson (2005) addressed several of these problems and suggested possible solutions (Table 3).

\section{EFFECTIVENESS OF STRUCTURES}

\section{Placement}

There are several challenges associated with designing and implementing wildlife crossing structures. In some instances, the location of crossing structures is very important and may be the most important factor (Podloucky 1989, Foster and Humphrey 1995, Yanes et al. 1995, Land and Lotz 1996, Rodriguez et al. 1996, Clevenger and Waltho 2000). This can be especially true for smaller, less mobile species such as reptiles and amphibians (Jackson and Griffin 2000). Rodriguez et al. (1996) suggested that crossing structures need to be placed in areas of suitable habitat and that passages 
implemented near continual disturbance (e.g. excessive human presence) were less frequented by several species.

\section{Dimensions}

The dimensions of structures are another important factor in designing passageways for vertebrates (Ulbrich 1984, Ballon 1985 as cited in Yanes et al. 1995). The size and shape of a particular structure may be the determining factor for crossing success (Reed et al. 1975, Ballon 1985, Cain et al. 2003, Clevenger and Waltho 2005). In Europe, hourglass-shaped overpasses have been found to be used regularly by wild boar but not by red deer who become unnerved or "spooked" by the constriction at the center (Vassant et al. 1993 as cited in Forman et al. 2003). For some species, the amount of openness in a passage may be more important than size (Foster and Humphrey 1995, Clevenger and Waltho 2005). Structures along the Trans-Canada highway with high openness ratios (short in length, high and wide) strongly influenced passage by grizzly bears (Ursus arctos horribilis), wolves (Canis lupus), elk, and deer while more constrictive structures were frequented more often by black bears (Ursus americanus) and cougars (Felis concolor) (Clevenger and Waltho 2005). Tunnels that allow animals to see the other end have been positively correlated with use by some species (Rosell et al. 1997 as cited in Jackson and Griffin 2000). Conversely, some studies (Rodriguez et al. 1996, Clevenger and Waltho 1999) have suggested that smaller passages may be better for some small mammals. The survival instinct of prey species, such as small mammals, can limit crossing use and there has been some evidence of predators using crossings to capture prey (Hunt et al. 1987, Foster and Humphrey 1995). Some structure designs, i.e., exposed, restricted, and narrow, may reduce the effectiveness of escape 
mechanisms of prey species (Reed et al. 1975, Yanes, et al. 1995, and Clevenger et al. 2002).

\section{Approaches}

Approaches to structures are another important factor in wildlife crossing design and implementation (Veenbaas and Brandjes 1999, Clevenger and Waltho 2000). The availability of cover (or lack of) at the approach to a crossing structure can determine if a particular species will use it. Natural vegetation can enhance the "attractiveness" of crossing structures to animals and allow a continuity of habitat. Cover may influence the use of crossings by small to mid-sized mammals (Hunt et al. 1987, Rodriguez et al. 1996, Clevenger and Waltho 1999) but deter other species such as deer and other ungulates if it is too restrictive (Pedevillano and Wright 1987, Clevenger and Waltho 2000).

\section{Fencing and Directional Devices}

The use of fencing and/or barrier walls along with passages is often needed to prevent animal access to the road and facilitate movement towards crossing structures (Ratcliffe 1983, Feldhamer et al. 1986, Jackson and Tyning 1989, Jackson 1996, AMBS 1997, Bissonette and Hammer 2000, Jackson and Griffin 2000, Dodd 2004, and Cavallaro et al. 2005; Figure 8). The use of a barrier wall in conjunction with a culvert system reduced roadkill in the Paynes Prairie State Preserve, Florida, by 93.5\% (Dodd 2004; Figure 9). For many larger species, fencing is necessary because of their inherent avoidance of passages. Many ungulates will avoid underpasses unless there is no other way to cross (Ward, 1982) and mountain lions traveling along streams have been known to leave the stream and cross over highways rather than use under-road culverts (Beier 1995). Fencing alone without crossing structures can be detrimental as it can act as a 
barrier to natural movements and contribute to habitat fragmentation (Jaeger and Fahrig 2004). Fencing also needs to extend far enough to either side of a crossing structure to promote guidance to the structure. The length of fencing is often dictated by the target species and the surrounding terrain.

\section{Structure Conditions}

Moisture, temperature, light, substrate, and noise (disturbance) can all be factors in determining if species will use wildlife passages (Langton 1989, Mansergh and Scotts 1989, Beier 1995, Yanes et al. 1995, Jackson 1996). Amphibians generally need moist conditions during migration so the ability to design passages that can allow rain to moisten the passage is important (Jackson 1996). Langton (1989) found that temperature differences between the interior and exterior of culverts may dissuade use by some amphibian species. The ability for air to flow free through a passage (e.g. grate tops rather than solid tops) may help to negate temperature differences and allow freer use. Moreover, open tops will allow more ambient light to enter crossing structures. Jackson and Tyning (1989) noted that increased natural light in tunnels accelerated the rate at which spotted salamanders (Ambystoma maculatum) would cross. Conversely, artificial light may often deter animals from a crossing structure (Reed et al. 1981, Jackson 2000).

The inclusion of a natural substrate within a crossing structure can provide continuity of habitat and may encourage animals to pass (Yanes et al. 1995, Jackson 2000). In controlled experiments between bare concrete tunnels, soil-lined tunnels, and open grass, Lesbarreres et al. (2004) found that water frogs (Rana esculenta) and common toads (Bufo bufo) preferred the tunnels to the grass while the agile frogs (Rana dalmatina) preferred grass. Use and crossing success were both higher in the soil-lined 
tunnel. There has been a suggestion that frogs are deterred from bare concrete due to its alkalinity (Mougey as cited in Lesbarreres 1996). Juvenile Western toads (Bufo boreas) and red-legged frogs (Rana aurora) showed greater movement in culverts with substrate versus culverts without (Bernard 2000 as cited in Fitzgibbon 2001).

Consideration of noise-reducing materials when constructing crossing structures is important to their success. The amount of noise (e.g. traffic) can affect animal usage of crossing structures (Clevenger and Waltho 2000, Clevenger and Waltho 2005, Jackson 2000). In Banff National Park, Canada, carnivore and ungulate movement through passages near the town of Banff was significantly affected by human activity and noise (Clevenger and Waltho 2000).

\section{CONCLUSION}

There are many ways to mitigate wildlife road mortality. Before planning any mitigation project various methods should be researched and all suggestions considered. Ultimately, preconstruction planning is more economical than retrofitting existing roads. When deciding to build wildlife crossing structures cost and effectiveness need to be addressed, but an understanding of various species and their requirements is just as important. Moreover, a solid post-construction monitoring program is essential to determine the success of any mitigation project, keeping in mind that animals need time to acclimate to any new structures. Furthermore, researchers and developers of wildlife road mortality mitigation methods should strive to move forward as engineering technology and understanding of biological systems advance. These types of discrepancies are the inherent problem with trying to design effective wildlife crossing 
structures; the need to accommodate the most species and yet be economical and structurally sound to the designer/builder.

\section{REFERENCES}

Aberg, L. 1981. The human factor in game-vehicle accidents: a study of driver's information acquisition. Acta Universitatis Upsalienis, Studia Psychologica Upsaliensia 6, Uppsala, Finland.

AMBS Consulting. 1997. Fauna usage of three underpasses beneath the F3 freeway between Sydney and Newcastle. Final report to New South Wales Roads and Traffic Authority. Sydney, Australia.

Aresco, M. J. 2005. Mitigation measures to reduce highway mortality of turtles and other herpetofauna at a north Florida lake. Journal of Wildlife Management 69:549-560.

Ballon, P. 1985. Premieres observations sur l'efficacite des passages a gibier sur l'autoroute A36. pp. 311-316 In Routes et faune sauvage. Service d'Etudes Techniques de Routes et Autoroutes, Bagneaux, France.

Bank, F. G., C. L. Irwin, G. L. Evink, M. E. Gray, S. Hagood, J. R. Kinar, A. Levy, D. Paulson, B. Ruediger, R. M. Sauvajot, D. J. Scott, and P. White. 2002. Wildlife habitat connectivity across European highways. Technical report FWHA-PL-02011. US Department of Transportation, Washington, D. C., USA.

Beier, P. 1995. Dispersal of juvenile cougars in fragmented habitat. Journal of Wildlife Management 59:228-237.

Bekker, H., B. van den Hengel, H. van Bohemen, and H. van der Sluijs. 1995. Natuur over wegen. Delft, Netherlands: Ministry of Transport, Public Works and Water Management.

Bernard, D. 2000. Unpublished environmental monitor's report submitted to the Vancouver Island highway project. British Columbia Ministry of Transportation and Highways. Victoria.

Bissonette, J., and M. Hammer. 2000. Comparing the effectiveness of earthen escape ramps with one-way gates in Utah. Unpublished. USGS Utah cooperative Fish and Wildlife Research Unit, Department of Fisheries and Wildlife, College of Natural Resources, Utah State University.

Cain, A. T., V. R. Tuovila, D. G. Hewitt, and M. E. Tewes. 2003. Effects of a highway and mitigation projects on bobcats in Southern Texas. Biological Conservation 114:189-197. 
Canadian Parks and Wilderness Society. 2004. http://www.cpaws.org/index.php.

Case, R. M. 1978. Interstate highway roadkilled animals: a data source for biologists. Wildlife Society Bulletin 6:8-13.

Clevenger, A. P., 1998. Permeability of the Trans-Canada Highway to wildlife in Banff National Park: importance of crossing structures and factors influencing their effectiveness. pp. 109-119 In Evink, G. L., P. Garrett, D. Zeigler, and J. Berry (eds) Proceedings of the international conference on wildlife ecology and transportation. FL-ER-69-98. Florida Department of Transportation, Tallahassee, Florida, USA.

Clevenger, A. P., B. Chruszcz, and K. Gunson. 2001. Drainage culverts as habitat linkages and factors affecting passage by mammals. Journal of Applied Ecology 38:1340-1349.

Clevenger, A. P., and N. Waltho. 1999. Dry drainage culvert use and design considerations for small- and medium-sized mammal movement across a major transportation corridor. pp. 263-277 In Evink, G. L., P. Garrett, and D. Zeigler. Proceedings of the third international conference on wildlife ecology and transportation. FL-ER-73-99. Florida Department of Transportation, Tallahassee, Florida, USA.

Clevenger, A. P., and N. Waltho. 2000. Factors influencing the effectiveness of wildlife underpasses in Banff National Park, Alberta, Canada. Conservation Biology 14:47-56.

Clevenger, A. P. 2004. Banff National Park of Canada website. http://www.pc.gc.ca/pn$\mathrm{np} / \mathrm{ab} / \mathrm{banff} /$ docs/routes/chap1/sec1/routes1b_e.asp.

Clevenger, A. P., and N. Waltho. 2005. Performance indices to identify attributes of highway crossing structures facilitating movement of large mammals. Biological Conservation 121:453-464.

Dodd Jr., C. K. W. J. Barichivich, L. L. Smith. 2004. Effectiveness of a barrier wall and culverts in reducing wildlife mortality on a heavily traveled highway in Florida. Biological Conservation 118:619-631.

Donaldson, B. M. 2005. Use of highway underpasses by large mammals and other wildlife in Virginia and factors influencing their effectiveness. pp. 433-441 In 2005 ICOET Proceedings.

Ellenberg, H., K. Muller, and T. Stottele. 1991. Strassen-Okologie. Pp 19-115 in Okologie und strasse. Broschurenreihe de Deutschen Strassenliga, Bonn, Germany. 
Emlen, S. T. 1975. Migration: orientation and navigation. pp. 129-219 In Farmer, D. S., and J. R. King (eds) Avian Biology, Volume 5. Academic Press, New York, NewYork, USA.

Evink, G. L., P. Garrett, D. Zeigler, and J. Berry (eds). 1996. Trends in addressing transportation related wildlife mortality. FL-ER-58-96. Florida Department of Transportation, Tallahassee, Florida, USA.

Feldhamer, G. A., J. E. Gates, D. M. Harman, A. J. Loranger, and K. R. Dixon. 1986. Effects of interstate highway fencing on white-tailed deer activity. Journal of Wildlife Management 50:497-503.

Ferris, C. R. 1979. Effects of Interstate 95 on breeding birds in northern Maine. Journal of Wildlife Management 43:421-427.

Fitzgibbon, K. 2001. An evaluation of corrugated steel culverts as transit corridors for amphibians and small mammals at two Vancouver Island wetlands and comparative culvert trials. M.S. thesis, Royal Roads University, Vancouver, Canada.

Hunt, A., J. Dickens, and R. J. Whelan. 1987. Movement of mammals through tunnels under railway lines. Australian Zoologist 24:89-93.

Federal Highway Administration. 2002. FHWA website. http://www.fhwa.dot.gov/environment/greenerroadsides/sum02p3.htm.

Federal Highway Administration. 2002. Critter crossings: linking habitats and reducing roadkill. http://www.fhwa.dot.gov/environment/wildlifecrossings/index.htm.

Foresman, K. R. 2001. Small mammal use of modified culverts on the Lolo South project of western Montana. ICOET 2001 proceedings.

Forman, R. T. T. 1995. Land mosaics: the ecology of landscapes and regions. Cambridge University Press, Cambridge, United Kingdom.

Forman, R. T. T., D. S. Freidman, D. Fitzhenry, J. D. Martin, A. S. Chen, and L. E. Alexander. 1997. Ecological effects of roads: toward three summary indices and an overview fro North America. pp. 40-54 In Canters, K. (ed) Habitat fragmentation and infrastructure. Ministry of Transport, Public Works and Water Management, Delft, Netherlands.

Forman, R. T. T. 2000. Estimate of the area affected ecologically by the road system in the United States. Conservation Biology 14:31-35.

Forman, R. T. T., and L. E. Alexander. 1998. Roads and their major ecological effects. Annual Review of Ecology and Systematics 29:207-231.

Forman, R. T. T., D. Sperling, J. A. Bissonette, A. P. Clevenger, C. D. Cutshall, V. 
H.Dale, L. Fahrig, R. France, C. R. Goldman, K. Heanue, J. A. Jones, F. J.Swanson, T. Turrentine, and T. C. Winter. 2003. Road ecology; science and solutions. Island Press, Washington D. C., USA.

Foster, M. L., and S. R. Humphrey. 1995. Use of highway underpasses by Florida panthers and other wildlife. Wildlife Society Bulletin 23:95-100.

Groot Bruinderink, G. W. T. A. and E. Hazebroek. 1996. Ungulate traffic collisions in Europe. Conservation Biology 10:1059-1067.

Jackson, S. D. 1996. Underpass systems for amphibians. In Evink, G. L., P. Garrett, D. Zeigler, and J. Berry (eds) Trends in addressing transportation related wildlife mortality. FL-ER-58-96, Florida Department of Transportation, Tallahassee, Florida, USA.

Jackson, S. D. 2000. Overview of transportation related wildlife problems. In Evink, G. L., P. Garrett, and D. Zeigler (eds) Proceedings of the Third International Conference on Wildlife Ecology and Transportation. Florida Department of Transportation Tallahassee, Florida, USA.

Jackson, S. D., and C. R. Griffin. 2000. A strategy for mitigating highway impacts on wildlife. pp. 143-159 In Messmer, T. A., and B. West (eds) Wildlife and highways: seeking solutions to an ecological and socio-economic dilemma. The Wildlife Society.

Jackson, S. D., and T. F. Tyning. 1989. Effectiveness of drift fences and tunnels for moving spotted salamanders Ambystoma maculatum under roads. pp. 93-99 In Langton, T.E.S. (ed) Amphibians and roads, proceedings of the toad tunnel conference. ACO Polymer Products Ltd, Bedfordshire, England.

Jacobson, S. L. 2005. Mitigation measures for highway-caused impacts to birds. PSWGTR-191. USDA Forest Service General Technical Report.

Jaeger, J. A. G., and L. Fahrig. 2004. Effects of road fencing on population persistence. Conservation Biology 18:1651-1657.

Jones, M. 2000. Road upgrade, road mortality, and remedial measures: impacts on a population of eastern quolls and Tasmanian devils. Wildlife Research 27:289-296.

Kaye, D., R., J., K. M. Walsh, and C. C. Ross. 2005. Spotted turtle use of a culvert under relocated Route 44 in Carver, Massachusetts. pp. 426-432 In 2005 ICOET Proceedings.

Keller, V. 1999. The use of wildlife overpasses by mammals: results from infra-red video surveys in Switzerland, Germany, France, and the Netherlands. pp. 27-28 In $5^{\text {th }}$ IENE meeting report, Budapest, Hungary.

Land, D., and M. Lotz. 1996. Wildlife crossing designs and use by Florida panthers and 
other wildlife in Southwest Florida. pp. 323-328 In Evink, G. L., P. Garrett, D. Zeigler, and J. Berry (eds) Trends in addressing transportation related wildlife mortality. FL-ER-58-96, Florida Department of Transportation, Tallahassee, Florida, USA.

Langton, T. E. S., 2002. Measures to protect amphibians and reptiles from road traffic. pp. 223-248 In Sherwood, B., D. Cutler, and J. Burton (eds) Wildlife and roads: the ecological impact. Imperial College Press, London, England.

Langton, T. E. S. 1989a. Tunnels and temperature: results from a study of a drift fence and tunnel system at Henley-on-Thames, Buckinghamshire, England. pp. 145-152 In Langton, T.E.S. (ed) Amphibians and roads, proceedings of the toad tunnel conference. ACO Polymer Products Ltd, Bedfordshire, England.

Langton, T. E. S. 1989b. Amphibians and roads, proceedings of the toad tunnel conference. ACO Polymer Products Ltd, Bedfordshire, England. 202pp.

LaPoint, S. D., R. W. Kays, and J. C. Ray. 2003. Animals crossing the Northway: are existing culverts useful? Adirondack Journal of Environmental Studies.

Lesbarreres, D., T. Lode, and J. Merila. 2004. Short communication: What type of amphibian tunnel could reduce road kills? Oryx 38:220-223.

Mansergh, I. M., and D. J. Scotts. 1989. Habitat continuity and social organization of the mountain pygmy-possum restored by tunnel. Journal of Wildlife Management 53:701-707.

Mougey, T. 1996. Des tunnels pour batraciens. Le Courier de la Nature 155:22-28.

National Research Council. 1997. Toward a sustainable future: addressing the long-term effects of motor vehicle transportation on climate and ecology. National Academy Press, Washington, D.C., USA.

Ogden, L., and J. Evans. 1996. Collision course: the hazards of lighted structures and windows to migrating birds. Report published by World Wildlife Fund Canada and Fatal Light Awareness Program. 46pp.

Pedevillano, C., and R. G. Wright. 1987. The influence of visitors on mountain goat activities in Glacier National Park, Montana. Biological Conservation 39:1-11.

Pfister, H. P., V. Keller, H. Reck, and B. Georgii. 1997. Bio-ecological effectiveness of wildlife overpasses or "green bridges" over roads and railway lines. Herausgegeben vom Bundesministerium fur Verkehr Abeteilung Strassenbau, Bonn-Bad Godesberg, Germany.

Podloucky, R. 1989. Protection of amphibians on roads: examples and experiences from Lower Saxony. pp. 15-28 In Langton, T.E.S. (ed) Amphibians and roads, 
proceedings of the toad tunnel conference. ACO Polymer Products Ltd, Bedfordshire, England.

Pojar, T. M., R. A. Prosence, D. F. Reed, and T. N. Woodard. 1975. Effectiveness of a lighted, animated deer crossing sign. Journal of Wildlife Management 39:87-91.

Puky, M. 2003. Amphibian mitigation measures in Central Europe. Proceedings of the International conference on ecology and transportation. Lake Placid, New York, USA.

Puky, M., and Z. Vogel. 2003. Amphibian mitigation measures on Hungarian roads: design, efficiency, problems, and possible improvement, need for a coordinated European environmental education strategy. Habitat fragmentation due to transportation infrastructure. IENE.

Ratcliffe, J. 1983. Why did the toad cross the road? Wildlife, August 304-307.

Reed, D. F. 1981. Mule deer behavior at a highway underpass exit. Journal of Wildlife Management 45:542-543.

Reed, D. F., T. N. Woodard, and T. M. Pojar. 1975. Behavioral response of mule deer to a highway underpass. Journal of Wildlife Management 39:361-367.

Reijnen, R., R. Foppen, C. T. Braak, and J. Thissen. 1995. The affects of car traffic on breeding bird populations in woodland, III. Reductions of density in relation to proximity of main roads. Journal of Applied Ecology 32:187-202.

Rodriguez, A., G. Crema, and M. Delibes. 1997. Use of non-wildlife passages across a high-speed railway by terrestrial vertebrates. Journal of Applied Ecology 33:1527-1540.

Romin, L.A., and J. A. Bissonette. 1996. Deer-vehicle collisions: status of state monitoring activities and mitigation efforts. Wildlife Society Bulletin 24:276-83.

Roof, J., and J. Wooding. 1996. Evaluation of the S.R. 46 wildlife crossing in Lake County, Florida. 7pp. In Evink, G. L., P. Garrett, D. Zeigler, and J. Berry (eds) Trends in addressing transportation related wildlife mortality. FL-ER-58-96, Florida Department of Transportation, Tallahassee, Florida, USA.

Rosell, C., J. Parpal, R. Campeny, S. Jove, A. Pasquina, and J.M. Velasco. 1997. Mitigation of barrier effect of linear infrastructures on wildlife. pp. 367-372 In Canters, K. (ed) Habitat fragmentation and infrastructure. Ministry of Transport, Public Works and Water Management, Delft, Netherlands.

Stoner, D. 1925. The toll of the automobile. Science 61:56-57.

Taylor, B. D. and R. L. Goldingay. 2003. Cutting the carnage: wildlife usage of road culverts in north-eastern New South Wales. Wildlife Research 30:529-537. 
Trombulak, S. C, and C. A. Frissell. 2000. Review of ecological effects of roads on terrestrial and aquatic communities. Conservation Biology 14:18-30.

Ulbrich, P. 1984. Untersuchung der wirksamkeit von wildwarnreflektoren und der eignung von wilddurchschlassen. Z. Jagdwiss 30:101-116.

Van Wieren, S. E., and P. B. Worm. 2001. The use of a motorway wildlife overpass by large mammals. Netherlands Journal of Zoology 51:97-105.

Vassant, J, S. Brandt, and J. M. Jullien. 1993a. Influence du passage de l'autoroute A5 sur les populations cert et sanglier du Massif d'Arc-en-Banois: 1er partie. Bulletin de l'Office National de la Chasse 183:15-25.

Vassant, J, S. Brandt, and J. M. Jullien. 1993b. Influence du passage de l'autoroute A5 sur les populations cert et sanglier du Massif d'Arc-en-Banois: 2eme partie. Bulletin de l'Office National de la Chasse 184:24-33.

Veenbaas, G., and J. Brandjes. 1999. Use of fauna passages along waterways under highways. pp. 253-258 In Evink, G. L., P. Garrett, and D. Zeigler (eds) Proceedings of the Third International Conference on Wildlife Ecology and Transportation. FL-ER-73_99. Florida Department of Transportation, Tallahassee, Florida, USA.

Ward, A. L. 1982. Mule deer behavior in relation to fencing and underpasses on Interstate 80 in Wyoming. Transportation Research Record 859:8-13.

Wildlife Crossings Toolkit. 2005. http://www.wlidlifecrossings.info/beta2.htm.

Woods, J. G. 1990. Effectiveness of fences and underpasses on the Trans-Canada Highway and their impact on ungulate populations. Report to Banff National Park Warden Service. Banff, Alberta, Canada.

Yanes, M., J. Velasco, and F. Suarez. 1995. Permeability of roads and railways to vertebrates: the importance of culverts. Biological Conservation 71:217-222. 
Table 1. Wildlife passage monitoring studies (modified from Forman et al. 2003).

\begin{tabular}{|c|c|c|c|c|c|}
\hline Study & Mitigation Measure & Location & $\begin{array}{l}\text { Target Species } \\
\text { (or group) }\end{array}$ & Monitoring Duration & $\begin{array}{l}\text { Species most often } \\
\text { encountered }\end{array}$ \\
\hline $\begin{array}{l}\text { AMBS } \\
\text { Consulting } 1997\end{array}$ & underpasses & $\begin{array}{l}\text { New South Wales, } \\
\text { Australia }\end{array}$ & unspecified & 9 months in 1997 & unspecified \\
\hline Aresco 2005 & drift fence and culverts & Florida, USA & reptiles and amphibians & Apr 2000 - Nov 2003 & reptiles and amphibians \\
\hline Ballon 1985 & unspecified & Upper Rhine, France & unspecified & 9 months in 1985 & ungulates \\
\hline Cain et al. 2003 & $\begin{array}{l}\text { bridges and culverts } \\
\text { underpasses and }\end{array}$ & Texas, USA & bobcats & Aug 1997 - May 1999 & bobcats \\
\hline $\begin{array}{l}\text { Clevenger } 1998 \\
\text { Clevenger and }\end{array}$ & overpasses & Alberta, Canada & $\begin{array}{l}\text { unspecified } \\
\text { small- and med-sized }\end{array}$ & $\begin{array}{l}\text { Jan } 1998 \text { - Dec } 1998 \\
74 \text { days in late }\end{array}$ & large mammals \\
\hline $\begin{array}{l}\text { Waltho } 1999 \\
\text { Clevenger and }\end{array}$ & dry drainage culverts & Alberta, Canada & mammals & $\begin{array}{l}\text { winter/early spring } \\
\text { Jan } 1995 \text { - Mar 1996, }\end{array}$ & weasels \\
\hline $\begin{array}{l}\text { Waltho } 2000 \\
\text { Clevenger and }\end{array}$ & $\begin{array}{l}\text { underpasses, culverts } \\
\text { underpasses and }\end{array}$ & Alberta, Canada & large mammals & Nov 1996 - Jun 1998 & elk \\
\hline Waltho 2005 & overpasses & Alberta, Canada & large mammals & Nov 1997 - Aug 2000 & deer \\
\hline Dodd et al. 2003 & culverts & Florida, USA & unspecified & Mar 2001 - Mar 2002 & Southern leopard frogs \\
\hline Donaldson 2005 & underpasses & Virginia, USA & $\begin{array}{l}\text { large mammals } \\
\text { amphibians and small }\end{array}$ & Jun 2004 - May 2005 & white-tailed deer \\
\hline Fitzgibbon 2001 & culverts & Vancouver, Canada & mammals & 2000 & weasels \\
\hline $\begin{array}{l}\text { Foresman } 2001 \\
\text { Foster and }\end{array}$ & culverts & Montana, USA & small mammals & $\begin{array}{l}\text { Jan 2001-Aug } 2001 \\
2 \text { month, } 16 \text { days in }\end{array}$ & unspecified \\
\hline Humphrey 1995 & underpasses & $\begin{array}{l}\text { Florida, USA } \\
\text { New South Wales, }\end{array}$ & Florida panthers & 1995 & medium- to large mammals \\
\hline Hunt et al. 1987 & tunnels & Australia & unspecified & 2 months in 1987 & small to medium-sized mammals \\
\hline $\begin{array}{l}\text { Jackson } 1996 \\
\text { Jackson and }\end{array}$ & amphibian tunnels & Massachusetts, USA & spotted salamanders & Spring 1998 & spotted salamanders \\
\hline Tyning 1989 & drift fences and tunnels & Massachusetts, USA & $\begin{array}{l}\text { spotted salamanders } \\
\text { eastern quolls, Tasmanian }\end{array}$ & $\begin{array}{l}1988 \\
\text { October } 1990 \text { - April }\end{array}$ & spotted salamanders \\
\hline Jones 2000 & reflectors, ramps, pipes & Tasmania & devils & 1993 & unspecified \\
\hline
\end{tabular}




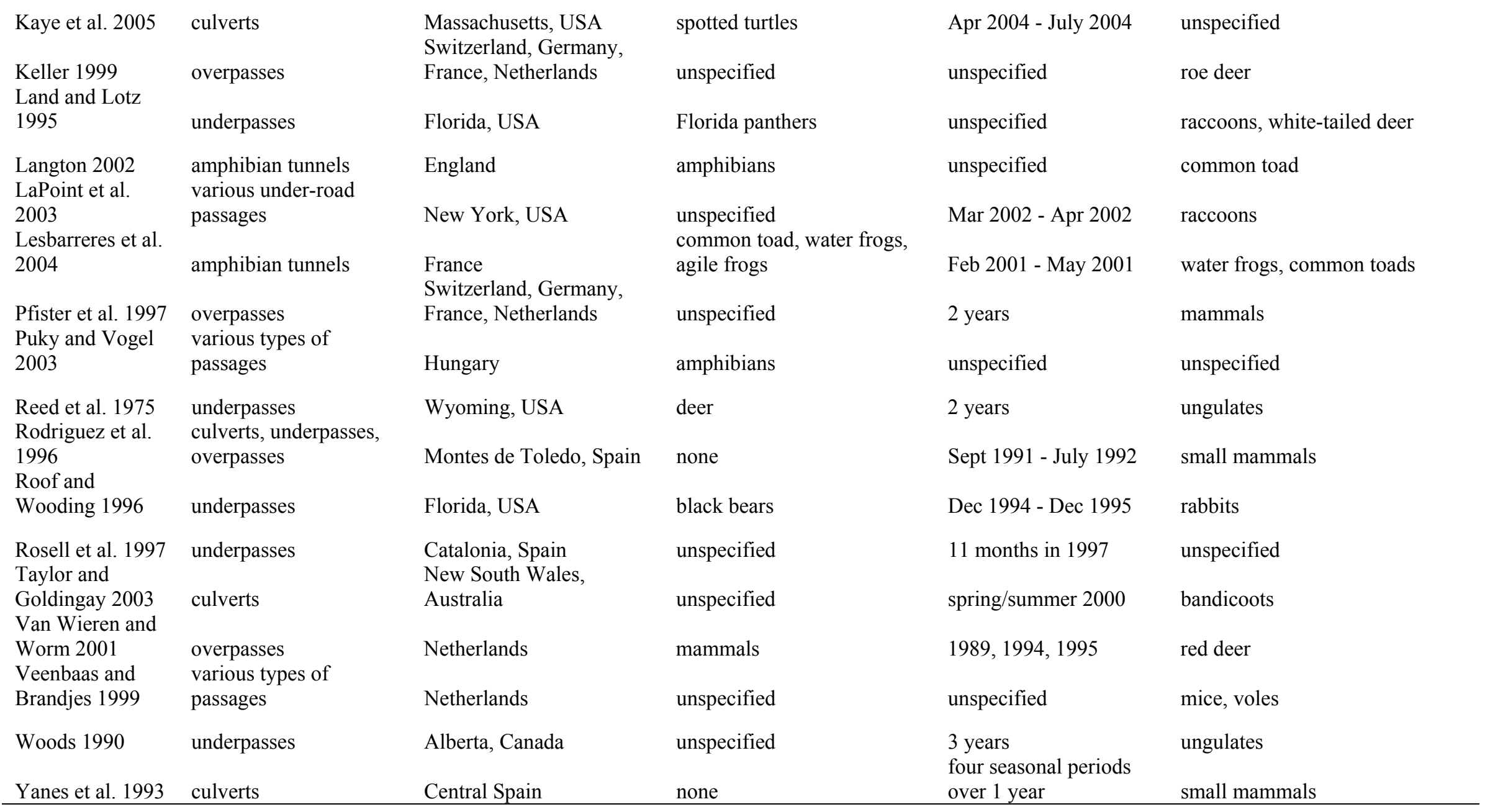


Table 2. Fragmentation and disturbance impacts to birds from highways (from Jacobson 2005).

\begin{tabular}{|c|c|c|}
\hline Impact & Problem & Suggested solution \\
\hline Loss of large carnivores & More small carnivores prey disproportionately on birds & Highway crossing structures for large carnivores \\
\hline Habitat dissection & Habitat parcels are too small to contain complete territories & $\begin{array}{l}\text { Avoid dissection by highway placement } \\
\text { Use causeways or viaducts to maintain small scale } \\
\text { habitat connectivity }\end{array}$ \\
\hline Isolation & Highways are barriers to less mobile or reclusive birds & $\begin{array}{l}\text { Overall connectivity strategy } \\
\text { Use open-span bridges, viaducts, or wildlife } \\
\text { overpasses }\end{array}$ \\
\hline Noise & Disrupts song or intimidates shy species & $\begin{array}{l}\text { Noise barriers } \\
\text { Reduce noise sources such as tires and road } \\
\text { surfaces }\end{array}$ \\
\hline Lights & Migrants can't see stars to navigate & $\begin{array}{l}\text { Coordinate light pollution reduction } \\
\text { Ensure lights are necessary before installation } \\
\text { Use lower wattage flat lens fixtures on highways, } \\
\text { retroreflective elements on signs and pavement }\end{array}$ \\
\hline
\end{tabular}


Table 3. Direct mortality to birds from highways (from Jacobson 2005).

\begin{tabular}{lll}
\hline Impact & Problem & Suggested solution \\
\hline Walking birds & Non-flying birds incur greater mortality risk & $\begin{array}{l}\text { Crossing structures with large openness ratios } \\
\text { (underpasses) or wildlife overpasses }\end{array}$ \\
Owls & Winds over bridges can slam flying birds into vehicles & Diversion poles on bridge decks \\
Ground nesters & Owls hunt at headlight level & $\begin{array}{l}\text { Diversion poles or short fences along highway } \\
\text { medians and rights-of-way }\end{array}$ \\
Scavengers & Killed while foraging on roadkill & Mow after August 1 \\
Migrant landfalls & Exhausted migrants fly into vehicles & $\begin{array}{l}\text { Reduce roadkill } \\
\text { Remove roadkill from road }\end{array}$ \\
Frugivores & Fruiting median plants attracts birds across traffic & $\begin{array}{l}\text { Low temporary fences to encourage higher flight } \\
\text { across roads }\end{array}$ \\
Winter finches & Deicing salt or sand attracts birds to road surface & $\begin{array}{l}\text { Plant non-fruiting varieties } \\
\text { Remove fruiting varieties }\end{array}$ \\
& & $\begin{array}{l}\text { Velocity spreaders } \\
\text { Road temperature sensors to reduce quantities } \\
\text { Concentrate runoff appropriately } \\
\text { Public education program }\end{array}$ \\
\hline
\end{tabular}


Figure 1. Small pipe culvert with mesh fence for small mammals and amphibians (Bank et al. 2002).

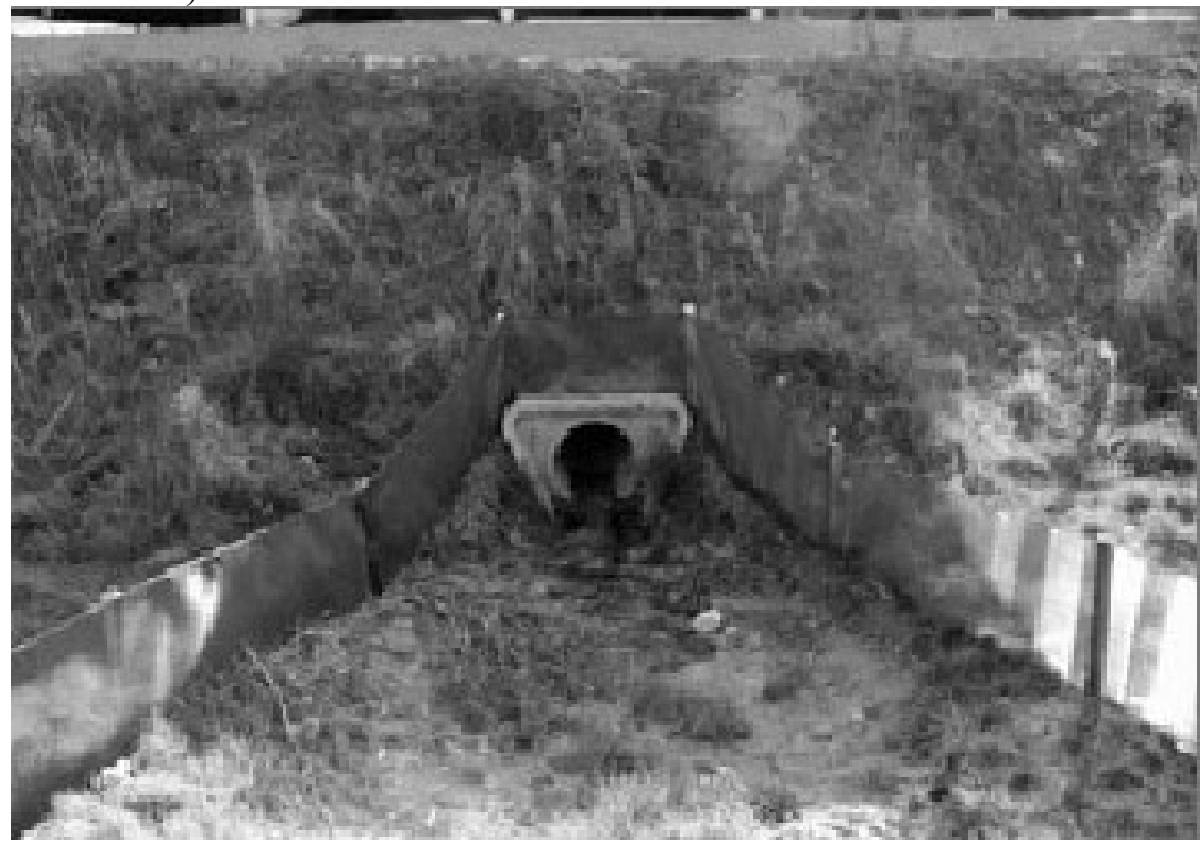

Figure 2. Amphibian tunnel (FWHA/USDOT 2002).

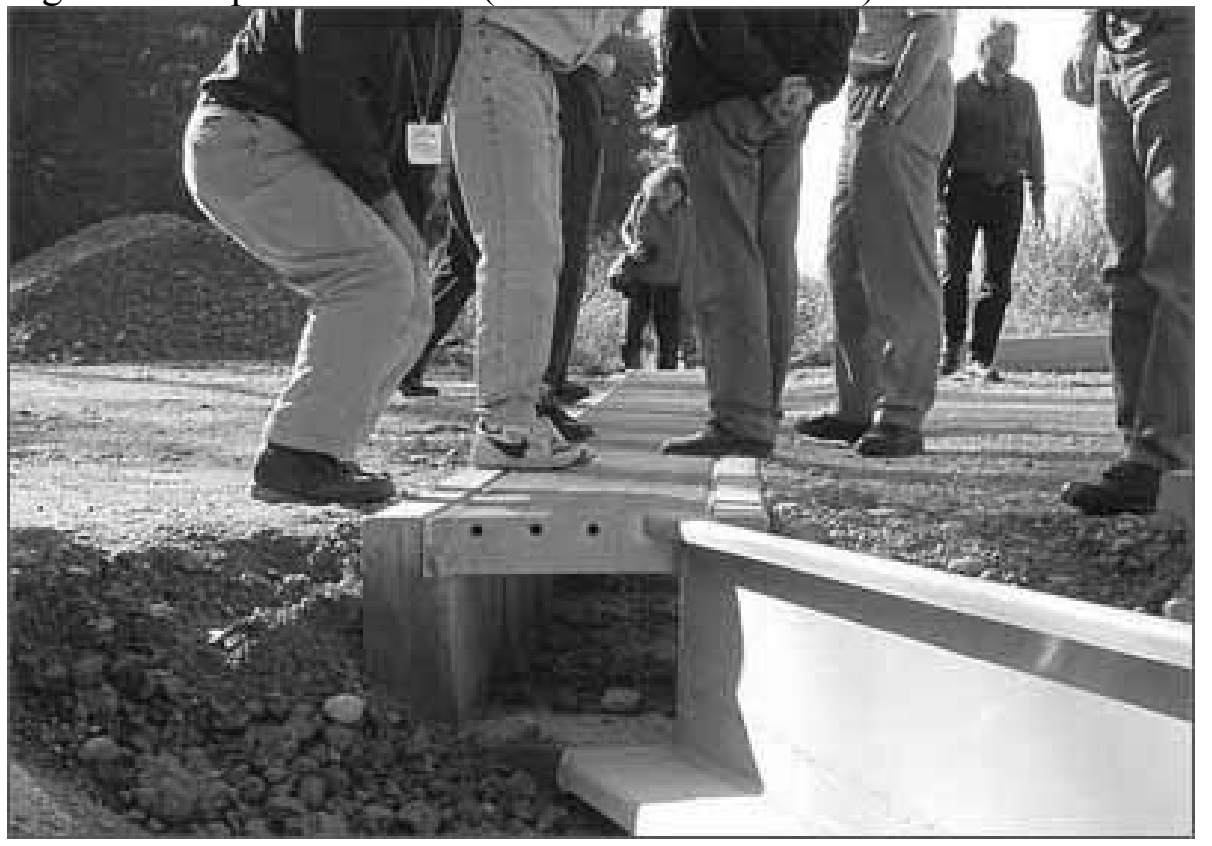


Figure 3. Box culvert and fencing (Clevenger 2004).

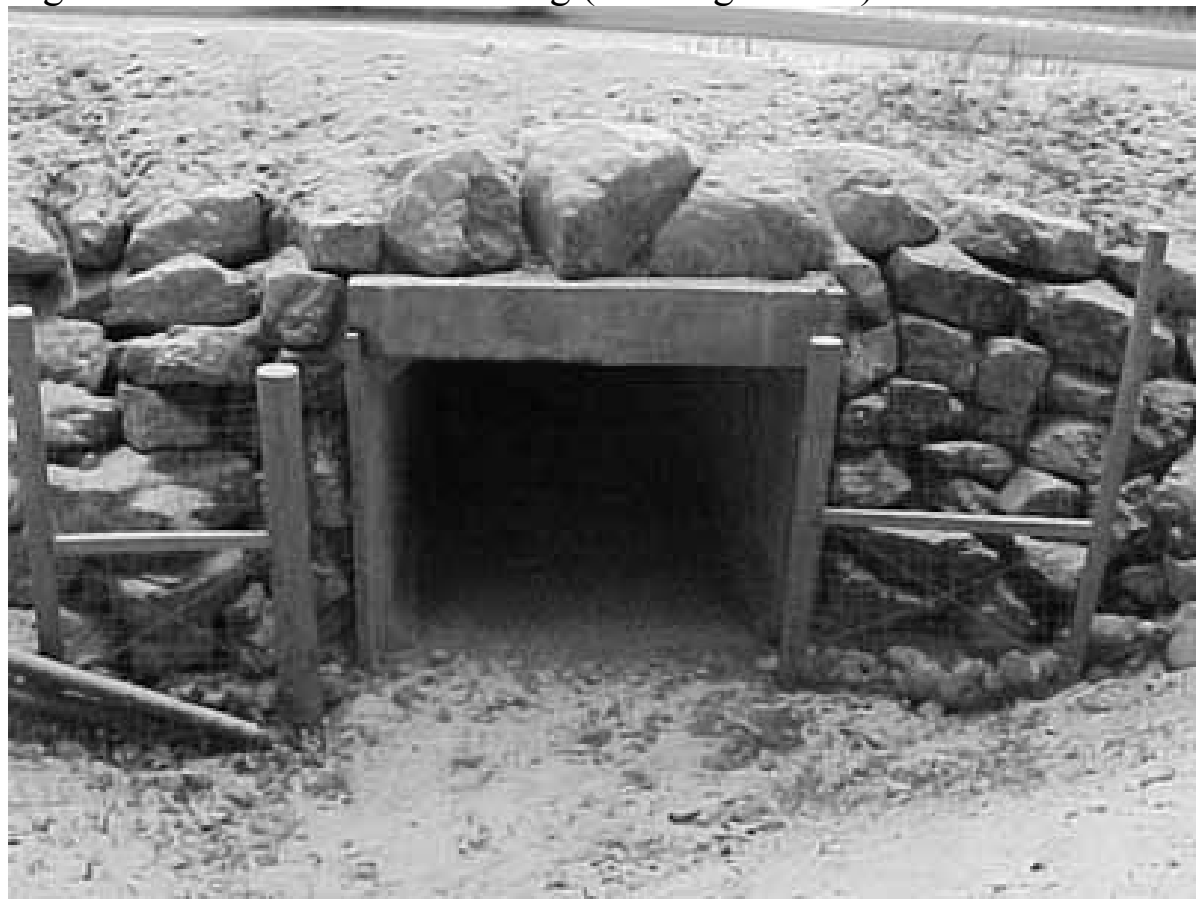

Figure 4. Box culvert underpass and fencing for multiple species (Puky 2003).

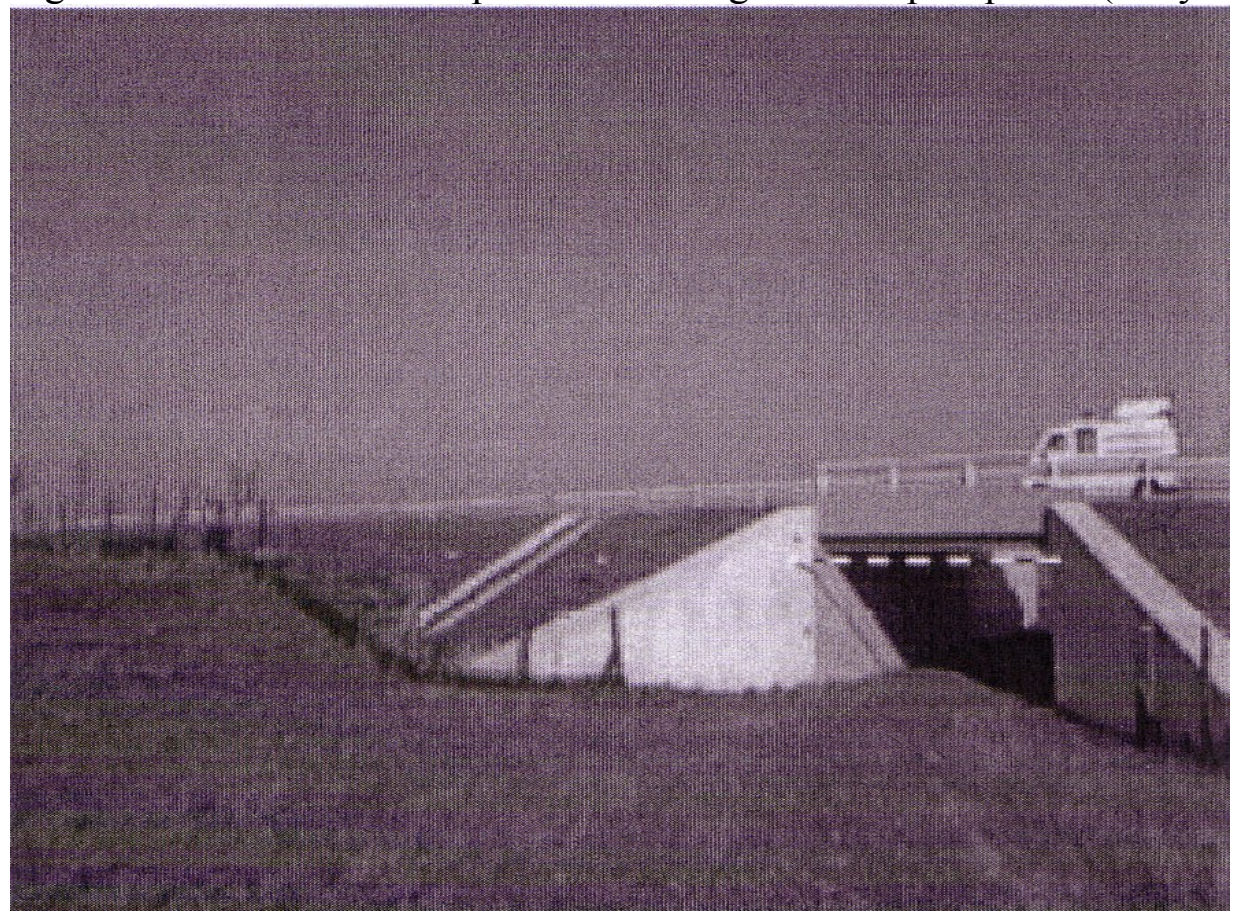


Figure 5. Creek underpass in Banff National Park, Canada (Clevenger 2004).

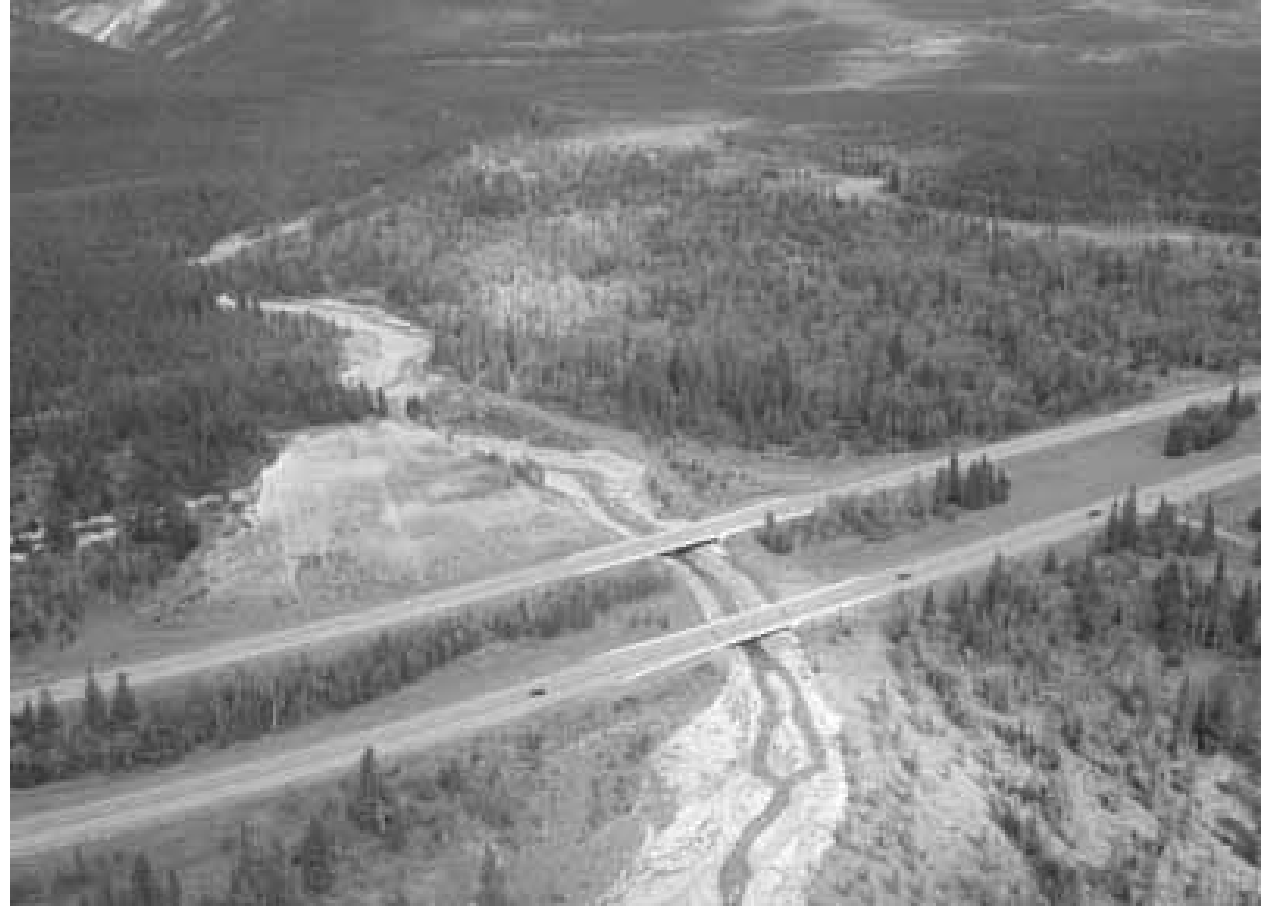

Figure 6. Box culvert modified with ledge for small animal passage (Bank et al. 2002).

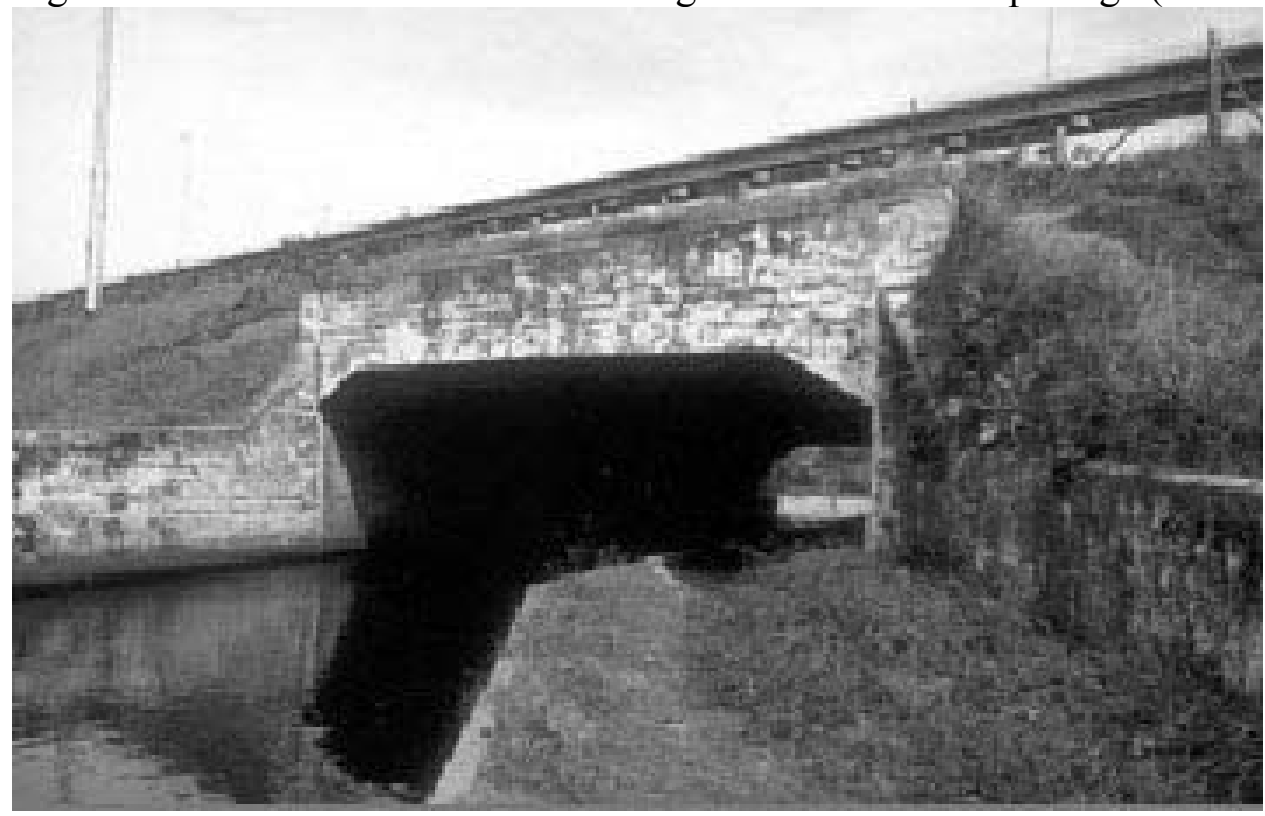


Figure 7. Overpass in Banff National Park, Canada (CPAWS 2004).

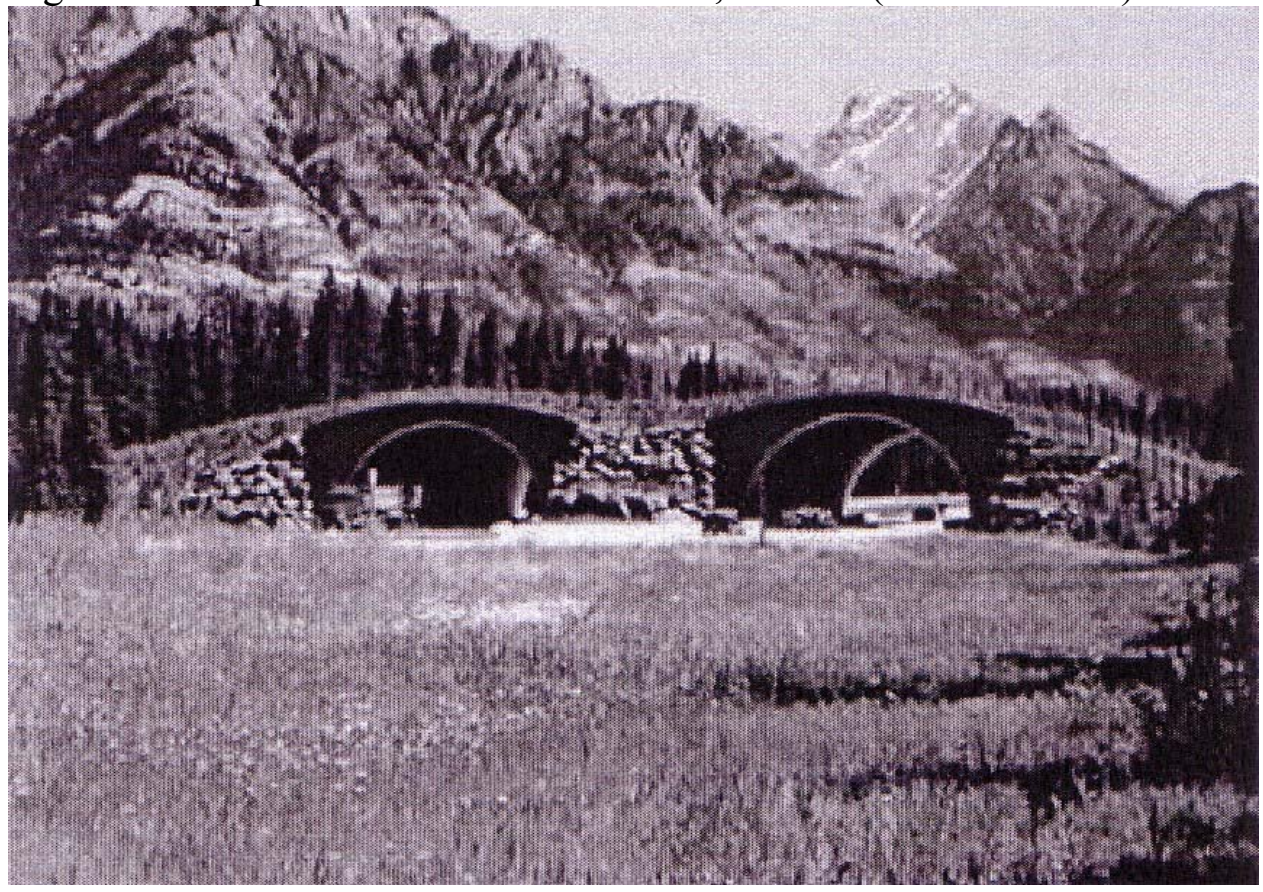

Figure 8. (Clevenger 2004)

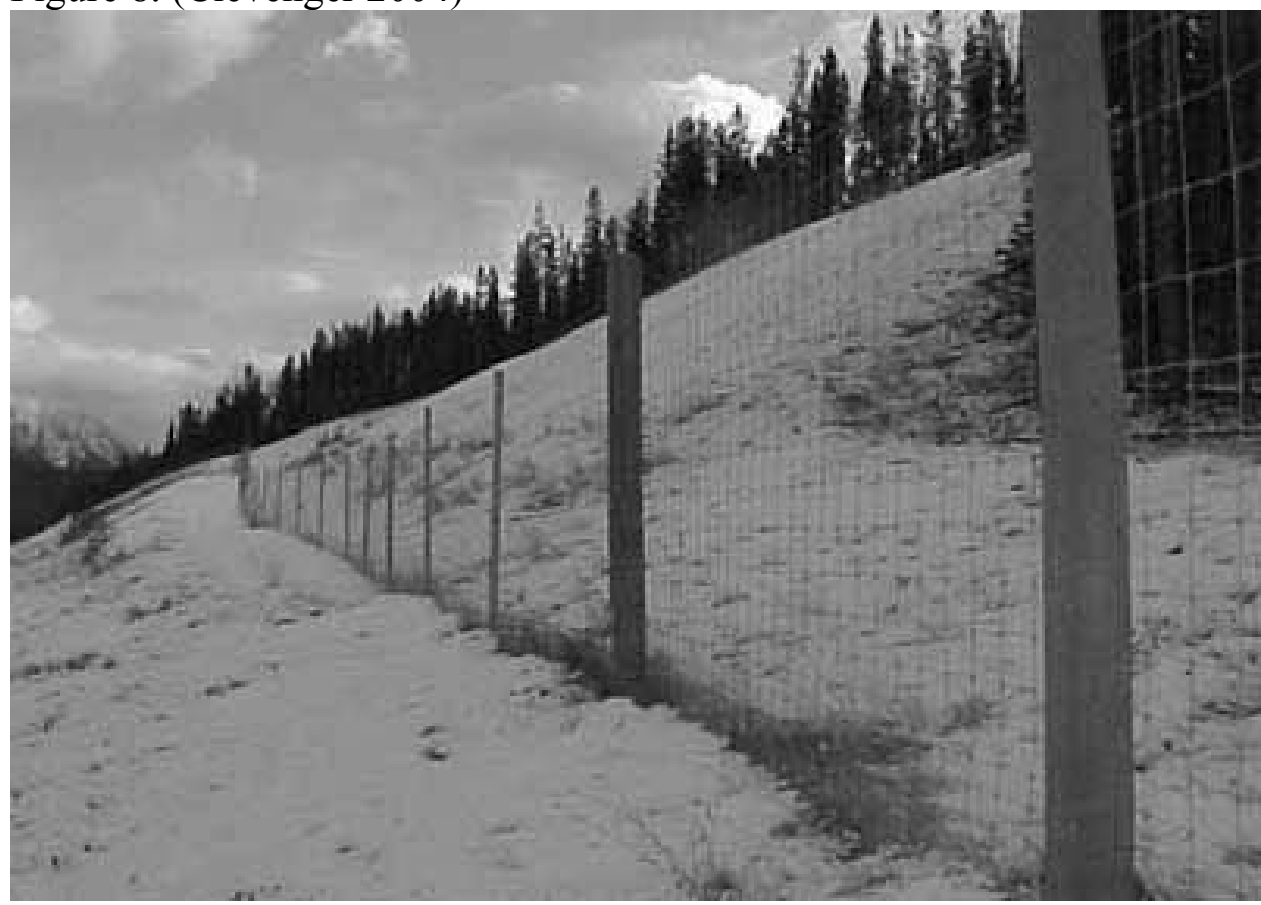


Figure 9. Amphibian wall and culvert system (FWHA/USDOT 2002).

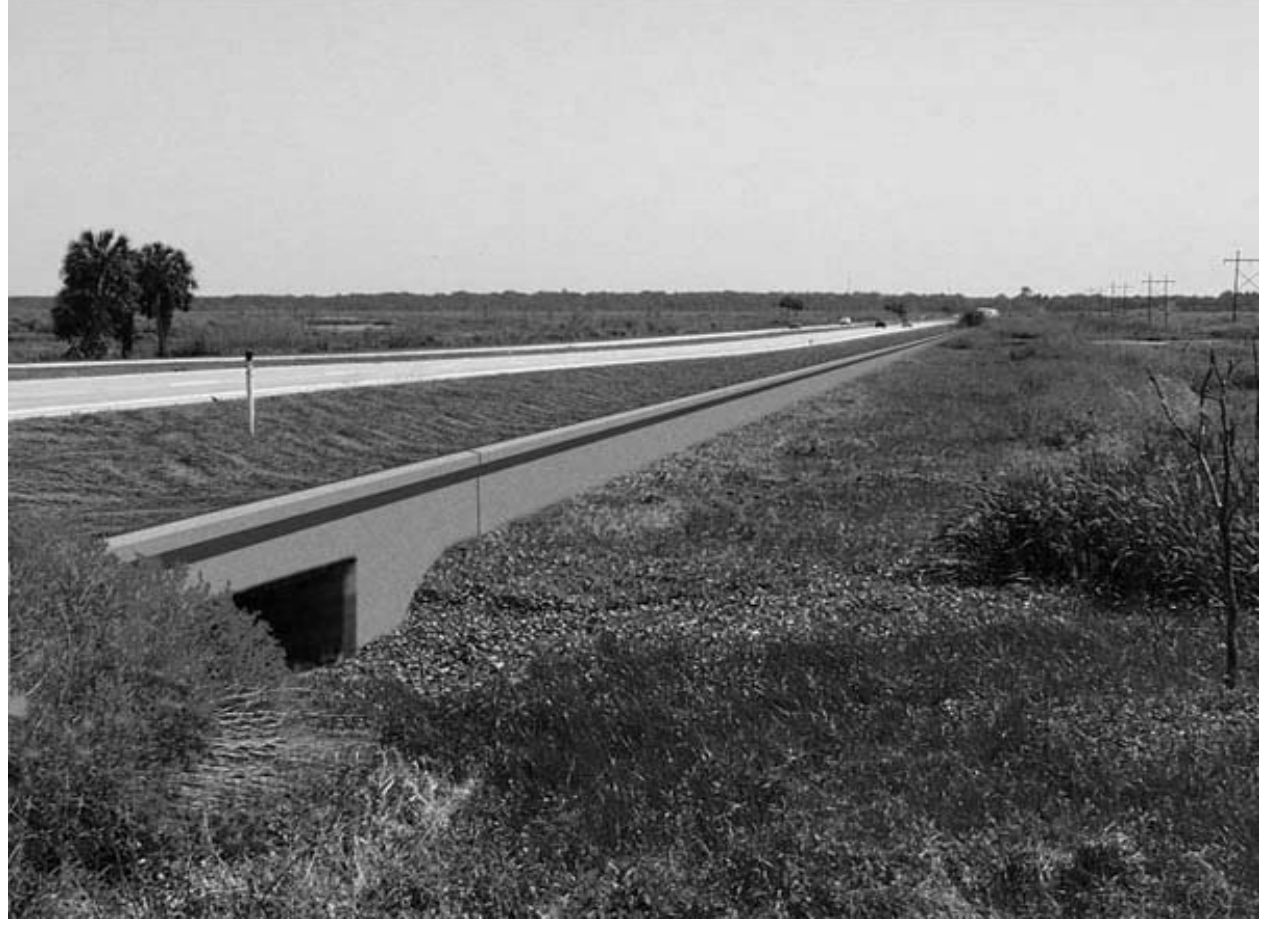


APPENDIX 


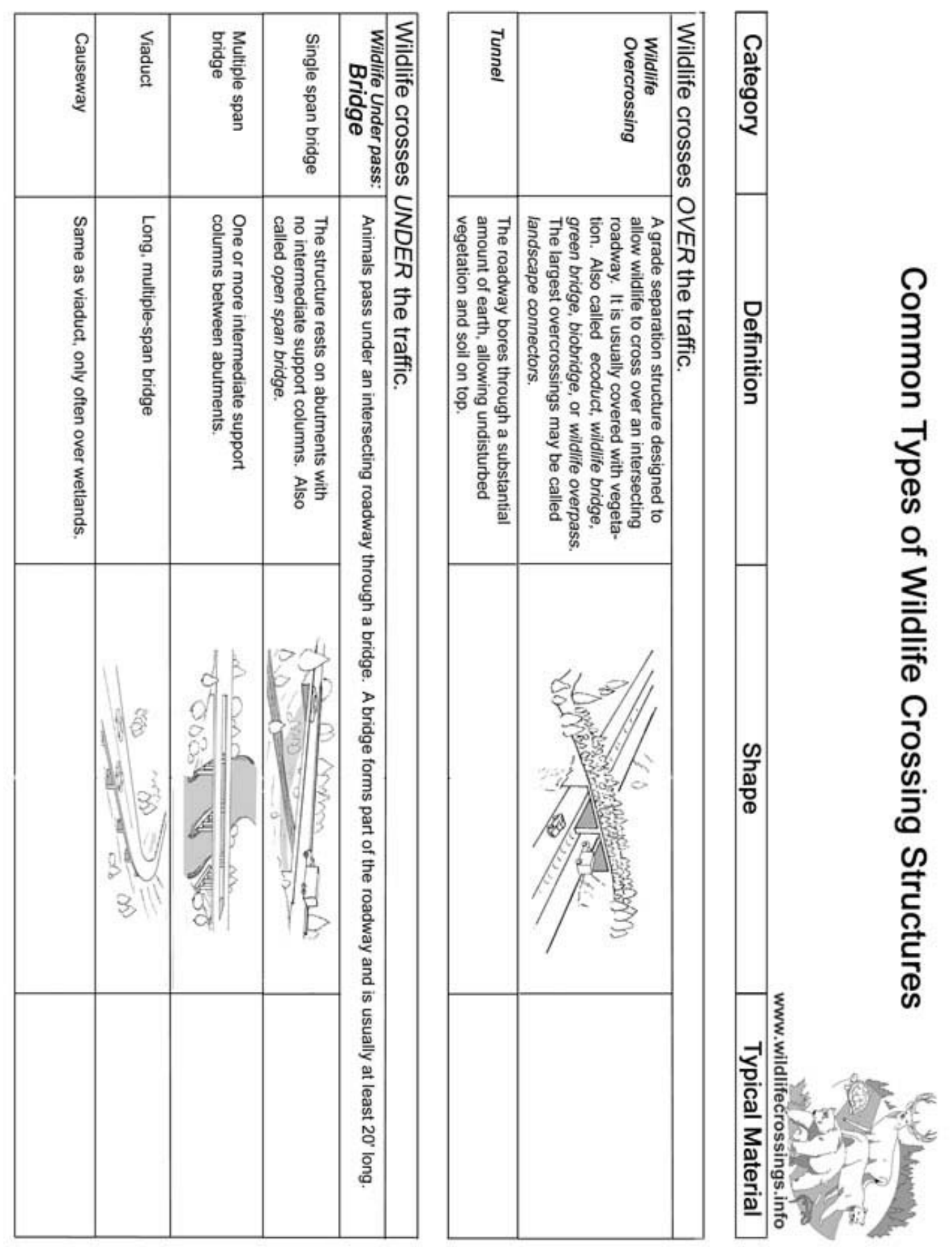




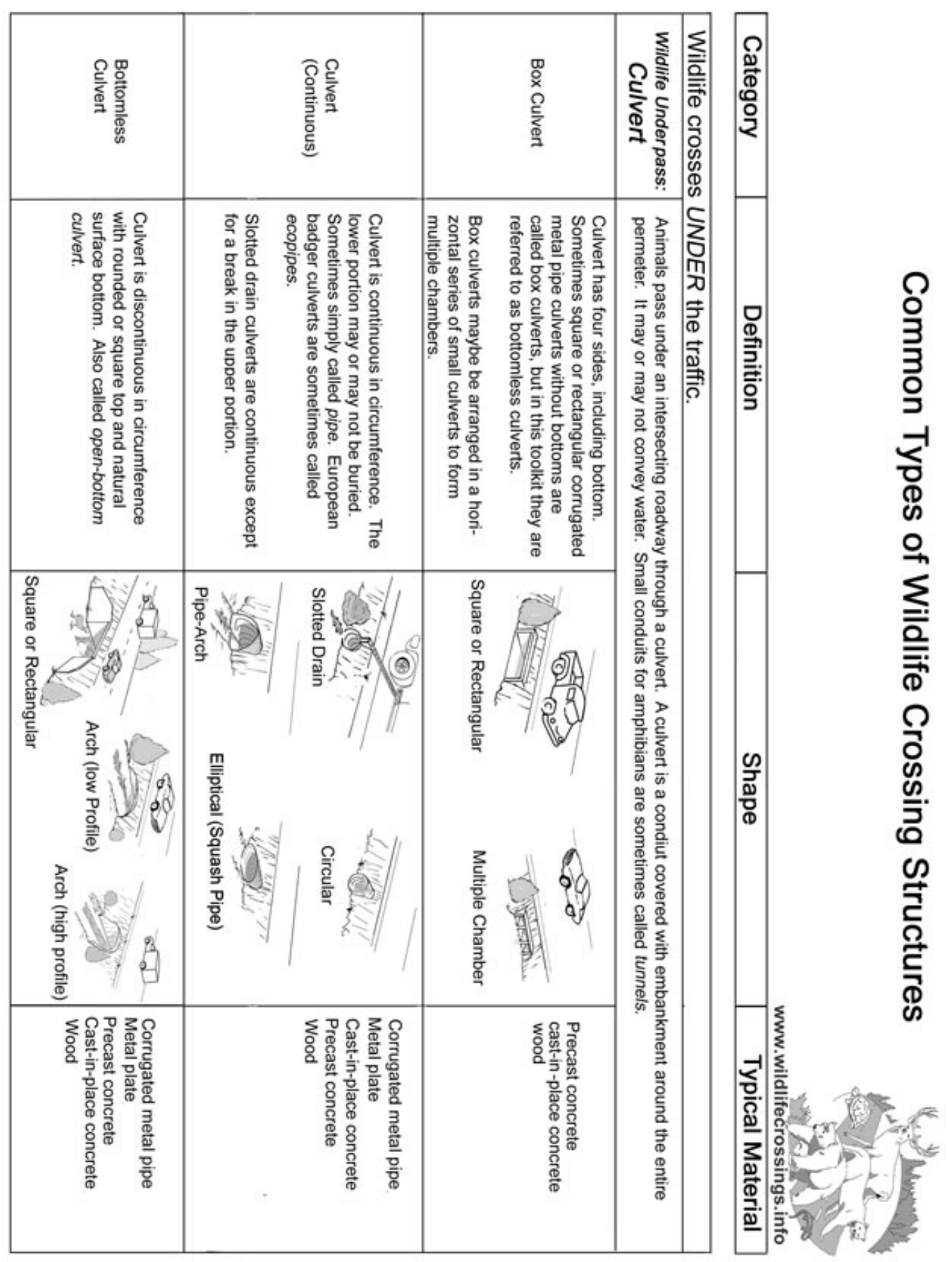




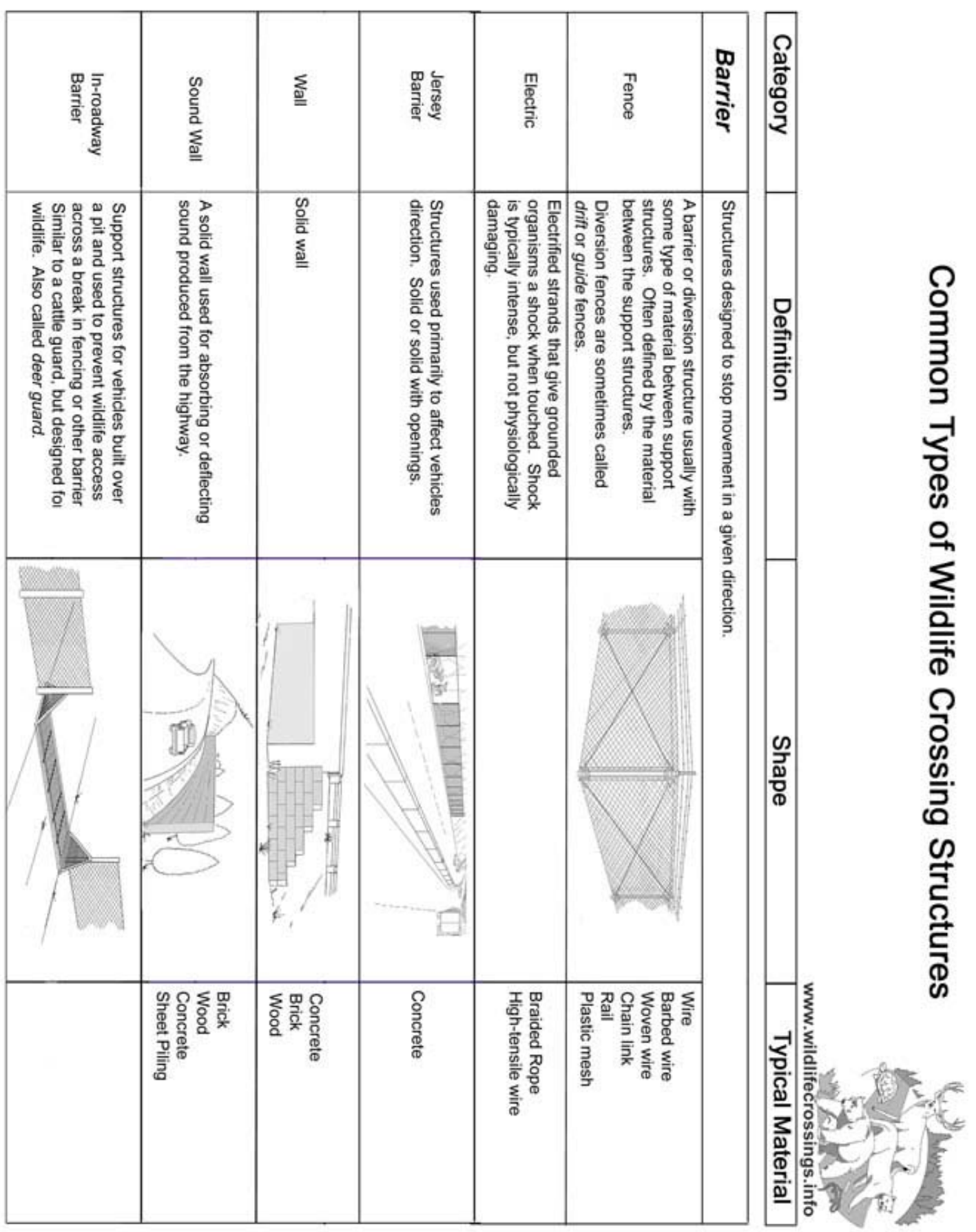




\section{CHAPTER 3. EVALUATION OF WILDLIFE HAZARDS AT GENERAL AVIATION AIRPORTS IN INDIANA}

\section{EXECUTIVE SUMMARY}

Collisions between wildlife and aircraft (wildlife strikes) are a serious problem facing wildlife managers, civilian aviation employees, and military personnel. Wildlife strikes have killed over 350 people worldwide and in the United States alone cause more than 580,000 hours of aircraft downtime and cost the civil aviation industry over $\$ 556$ million annually (Cleary et al. 2006). Despite a substantial amount of information available on the causes of wildlife strikes at large international airports, few researchers have considered wildlife hazards at smaller regional airports and airfields. In this study, we evaluated the need for management actions to reduce wildlife strikes at general aviation airports throughout Indiana by conducting wildlife hazard assessments at a subset of 10 airports throughout the state. For each focal airport, we conducted 1) habitat assessments in the airport environment (including perimeter-fence evaluations), 2) inventories of wildlife that could be hazardous to aircraft, and 3) surveys of pilot/airport operator perceptions regarding wildlife hazards.

On average, airport habitats consisted mainly of short grass (40.2\% of total airport area), soybean fields $(10.3 \%)$, corn fields $(9.5 \%)$, runway systems $(8.1 \%)$, other development (6.6\%), woodlots (5.2\%), medium grass (4.8\%), tall grass (4.6\%), and hayfields (3.2\%). At least two types of wildlife attractants were present at each airport property, but most airports had five to seven types. The most common wildlife attractants included standing water (ephemeral), open culverts, crop fields, woodlot refugia, and gravel piles. Proportion of airport perimeters fenced ranged from $7.5 \%$ to 
$100 \%$, but most airport perimeters were $>40 \%$ fenced. Most airports with $>25 \%$ of the perimeter enclosed by chain-link fencing had 0.2-0.5 openings per $100 \mathrm{~m}$ of fence, with gaps and dig-holes being the most common openings.

Considering the most hazardous species, $0-92$ white-tailed deer and $0-28$ coyotes were observed at individual airports combining all survey methods across a year. Of the 16 bird species groups identified by Dolbeer et al. (2000) as hazardous to aircraft, American kestrel, blackbirds-starling, crows-ravens, mourning dove, shorebirds, sparrows, and swallows were present at 9-10 of the airport properties; geese, hawks (buteos), and vultures were present at 7-8 of the airport properties; and ducks, herons, and rock doves were present at 5-6. Among all airports, the most numerous bird species group was blackbirds-starling, although the abundance of the blackbirds-starling group and most other species groups varied widely across seasons and airports.

Questionnaires indicated that pilots using focal airports clearly were accustomed to wildlife hazards: $69 \%$ of respondents reported that they had altered aircraft operation due to wildlife within the past year, and $25 \%$ reported involvement in a wildlife strike during the past year. Furthermore, $88 \%$ of respondents felt that wildlife populations at Indiana airports were at least "somewhat hazardous". Despite pilots' awareness of wildlife hazards, less than $70 \%$ of respondents supported the use of fencing or wildlife deterrents, $43 \%$ supported modification/elimination of wildlife habitat, and only $38 \%$ of respondents supported for lethal removal of wildlife on airport properties.

Based on our research at focal airports, we conclude that wildlife hazard management should be improved at general aviation airports in Indiana. Because most wildlife hazard problems at airports can be addressed with traditional methods, we 
recommend that airport personnel become familiar with established techniques, such as those summarized in Cleary and Dolbeer (1999). Hazards associated with deer and coyotes could be alleviated by installing suitable fencing where funds are available. For airports with extant fences, care should be taken to monitor fences regularly and repair gaps as soon as they are discovered. Presence of deer and coyotes inside airport fences should not be tolerated. Birds are best managed by maintaining airport habitats in a manner that minimizes availability and/or quality of food, water, cover, and loafing sites for hazardous species. Furthermore, several new technologies and refinements in techniques for wildlife damage management at airports have emerged recently and may benefit small airports, such as advancements in electric fencing and the use of dead bird effigies to repel some hazardous bird species. 


\section{INTRODUCTION}

Collisions between wildlife and aircraft (wildlife strikes) are a serious problem both for economic and safety reasons. Wildlife strikes cause more than 530,000 hours of aircraft downtime each year and cost the civil aviation industry over $\$ 500$ million annually (USDA/APHIS 2004). Furthermore, more than 100 people have died as a result of wildlife strikes in the U.S. since 1960 (Cleary and Dolbeer 1999), and over 350 people have been killed in wildlife strikes worldwide since the inception of aviation 100 years ago (Sodhi 2002). Unfortunately, the wildlife strike problem is expected to worsen, as 1) air travel increases, 2) wildlife populations grow, and 3) commercial air carriers replace three- and four-engine aircraft with quieter, more efficient two-engine aircraft that are more vulnerable to catastrophic strikes (Cleary et al. 2003).

In addition to wildlife strikes with civil aircraft, military aircraft also have experienced significant losses. Between 1990 and 1998, there were an estimated 22,000 collisions between birds and aircraft in the U.S.; such collisions cost $\$ 400$ million annually in aircraft repairs. In particular, aircraft of the U.S. Air Force incur over 2,500 bird strikes per year (Lovell 1997), and since 1987, 5 U.S. Air Force aircraft have been totally destroyed with 4 crewmen killed (Arrington 2003). It is clear that understanding the causal factors contributing to bird-aircraft collisions and developing solutions to reduce such collisions are critical challenges currently facing wildlife managers, civilian aviation employees, and military personnel.

Although mid-air collisions between aircraft and large soaring birds can be catastrophic (DeVault et al. 2005), collisions in the airport environment are more problematic overall. Commercial and general aviation airports, which commonly are 
located in close proximity to water bodies and large grasslands, often harbor large populations of birds, white-tailed deer, coyotes, and other wildlife that are potentially dangerous to aircraft (Dolbeer et al. 2000). The combination of abundant wildlife populations and frequent aircraft take-offs and landings at airports commonly leads to unacceptable levels of wildlife-strike occurrences—over $90 \%$ of wildlife strikes to civil aircraft occur in the airport environment (Cleary et al. 1999). Birds account for approximately $97 \%$ of all aircraft collisions with wildlife, and the vast majority of bird strikes occur in the airport environment. Mammals are also hazardous at airports; 616 white-tailed deer collisions and 174 coyote collisions were reported in the U.S. from 1990-2004 (Cleary et al. 2005). Since 1990, 172 collisions with birds ( 141; 82\%) and mammals $(31 ; 18 \%)$ have occurred in the United States, of which 9 resulted in human fatalities ( 8 bird and 1 mammal; Cleary et al. 2006). An increased understanding of the causal factors contributing to wildlife strikes at airports and the continued development of cost-effective solutions to reduce such collisions potentially would reduce human mortalities and substantial economic losses to the aviation industry.

Wildlife biologists have studied wildlife strikes in many locations, but most research has been limited to investigations at large international airports (e.g., Dolbeer et al. 1993); few researchers have considered wildlife problems at smaller regional airports and airfields. However, because smaller airports often are located in rural areas, the potential for wildlife strikes is usually significant. Some information is available concerning bird community structure at small airports in Illinois (Kershner and Bollinger 1996), but these surveys were concerned more with bird conservation than aviation hazards. Very little information is available regarding community structure of birds and 
large mammals at small midwestern airports. Nonetheless, every airport that receives grant-in-aid assistance from the Federal Aviation Administration (FAA), regardless of its size and the type of air traffic it accommodates, is required to ensure a safe operating environment with respect to wildlife hazards (Federal Aviation Administration Advisory Circular 150/5200-33A, 2004). Thus, it is necessary for all airports to sufficiently identify potential problems with hazardous wildlife to prevent wildlife strikes.

Cleary and Dolbeer (1999) prepared a manual with the purpose of assisting "airport personnel in the development, implementation, and evaluation of Wildlife Hazard Management Plans at airports." They demonstrated how to evaluate airport environments for wildlife hazards and how to implement appropriate management strategies. The problem of wildlife strikes varies from airport to airport, depending on factors such as air traffic type and volume, local and migratory wildlife populations, and local wildlife habitat conditions. Thus, for effective wildlife strike management to be implemented at a local scale, the nature and magnitude of wildlife strike problems must be evaluated at each airport individually. Simple management measures at airports often reduce wildlife strikes significantly, but local assessments of wildlife problems must be conducted first.

The objective of the research described herein was to evaluate the need for management actions to reduce wildlife strikes at general aviation airports throughout Indiana. In essence, we conducted a wildlife hazard assessment (see Cleary and Dolbeer 1999) at a subset of general aviation airports throughout the state. Ten focal airports were chosen for study and varied in size, aircraft traffic, habitat composition, and proximity to urban areas. For each focal airport, our wildlife hazard evaluations 
consisted of 1) inventories of wildlife that could be hazardous to aircraft, 2) habitat assessments in the airport environments, and 3) surveys of pilot/airport operator perceptions regarding wildlife hazards.

Wildlife inventories were designed to census the bird and mammal communities at each focal airport over a year. We conducted nighttime spotlight surveys and baited remote camera surveys to document the presence and relative abundance of deer, coyotes, and other medium-sized mammals (e.g., raccoons, opossums). We also conducted seasonal walking transect surveys to document the presence and abundance of birds on airport properties that are potentially dangerous to aviation.

We conducted two types of habitat assessments: on-site habitat evaluations and Geographic Information System (GIS) analyses. On-site evaluations allowed us to examine and map local features of airport properties that might influence wildlife presence, abundance, and/or activity patterns (e.g., open garbage receptacles, depressions in pavement that could collect water and attract birds) that were not evident from aerial photographs or other remote imagery, and to measure and evaluate airport fences. GIS analyses allowed us to assess airport habitats (and surrounding properties in some cases) in a more quantitative manner, by categorizing and measuring the extent and proportion of various habitat types present at the focal airports. GIS analyses also produced maps and associated data layers that were made available to airport managers.

To document wildlife hazard perceptions and experiences of pilots and airport operators at each airport in our subset, we designed and distributed a standardized questionnaire to airport managers at each focal airport. We gathered information on past 
wildlife strikes and near-strikes, perceptions about the importance of wildlife hazard management, and sightings of notable wildlife hazards.

\section{METHODS}

\section{$\underline{\text { Selection of Focal Airports }}$}

Ten airports were chosen as study sites for our investigation of wildlife hazards at Indiana airports (Table 1). Participating airports were chosen in consultation with the Indiana Department of Transportation (INDOT) and the Aviation Association of Indiana (AAI). We attempted to select our subset of airports so that it represented the spectrum of sizes, aircraft traffic, habitat types, proximity to large urban areas, and current extent of wildlife hazard management programs exhibited by Indiana airports. We visited each airport twice during each of 4 seasons (spring 2005, summer 2005, fall 2005, winter 2006) for a total of 8 visits per airport. During the first visits in spring 2005, we met with airport personnel to learn security procedures at each property and establish sampling locations for inventories of wildlife.

\section{Wildlife Inventories}

\section{Spotlight Surveys}

We conducted two spotlight surveys (Bookhout 1996) during each season at each airport, for a total of 8 spotlight surveys per airport. Survey routes were designed to cover as much of the airport property as practical, given the specific conditions present at each airport (i.e., habitat, topography, access), and ranged from 1.63-7.93 km in length (Table 1; Figs. 1-10). Spotlight surveys began between $0.5 \mathrm{hr}$ after sunset and 23:30 EST. During each survey, a team of 1-3 observers drove slowly $(\sim 10 \mathrm{~km} / \mathrm{h})$ in a truck or ATV along the established route, stopped frequently, and shined a 1,000,000 candle-power 
spotlight on both sides of the route. When an animal was observed, we recorded the species and distance from the survey route on a standardized data sheet.

\section{Bird Surveys}

We used walking line transect surveys (Bibby et al. 1992) to sample bird populations at each airport. We conducted two surveys during each season at each airport, for a total of 8 bird surveys per airport. Transect lengths ranged from 0.79-1.89 km (Table 1; Figs. 110), and were established based on: 1) ability to survey a representative sample of habitats available to birds within the airport property, 2) topography (in terms of our ability to view as much of the airport property as possible), and 3) accessibility. A team of 1-3 observers walked at a pace of $\sim 2 \mathrm{~km} / \mathrm{hr}$ and paused frequently to listen and look for birds. We were careful not to double-count individuals at corners on L- or U-shaped transects. During spring and summer seasons, counts were confined to a 5-hr period beginning 30 min before sunrise on days with little wind and no rain. During fall and winter (non-breeding season when birds do not sing), counts were not restricted to morning hours. For each bird detected, the observer recorded species, mode of detection (song, call, or visual), and distance to transect. Many birds that were counted were singing and, therefore, adult males. However, detections by sight or call note likely included some females, especially for visually conspicuous species such as red-winged blackbird and eastern meadowlark, so we report count data as "individuals" per km. Here, we report only the 16 species groups identified by Dolbeer et al. (2000) as potentially hazardous to aviation.

\section{Remote Camera Surveys}


We used digital infrared remote cameras (Stealth Cam, Inc.), designed to trigger upon movement, as an additional means of detecting wildlife on airport properties. Because we did not capture and mark individuals (e.g., individual deer and coyotes), it was not possible to assess absolute abundance of wildlife using remote cameras (i.e., use of remote cameras did not constitute a mark/recapture technique). Thus, we primarily used cameras to detect wildlife presence and compare relative abundances among airport properties, as well as to assess the effectiveness of perimeter fences. We placed cameras in locations where animals were likely to travel, such as fence holes, openings of culverts, small wetlands, refugia (e.g., small woodlots), and preexisting wildlife trails (Figs. 1-10). During each season at each airport, 3-4 cameras were placed on airport properties and baited with a commercial wildlife attractant (skunk essence). Each camera operated for a maximum of 240 hours during each of the spring, summer and fall seasons. In an effort to increase performance, we equipped cameras with larger batteries during the winter season, resulting in an average operating time of 890 hours per camera. Photographs were downloaded onto a laptop computer and analyzed after collection. We attempted to use the same camera locations each season, although occasionally it was necessary to establish new camera locations (e.g., when cameras were subject to flooding, tampering, or frequent anthropogenic activity).

\section{Opportunistic Observations}

During each site visit to airport properties, we were careful to record any noteworthy observation of wildlife, especially presence of species hazardous to aviation (e.g., vultures, gulls, geese, coyotes, and deer). Opportunistic observations were made using visual observations of individuals and by noting presence of scat and tracks. 


\section{$\underline{\text { Habitat and Fencing Evaluation }}$}

\section{On-site Analyses}

We conducted an on-site habitat assessment during the summer season at each airport and created a field map of major habitat types that could be expected to influence presence or abundance of wildlife on the airport property. We consulted aerial photographs (obtained by downloading geographic raster data generated in 2003 by the USDA National Agricultural Imagery Program [NAIP], provided by the Indiana Geological Survey [IGS; http://129.79.145.5/arcims/statewide/index.html]) to aid our interpretation of the spatial extent and location of habitat patches. If a given airport had a completely fenced airfield, we mapped habitats within the fenceline only. For airports that lacked complete fencing or other obvious boundary markers (e.g., roads), we mapped habitats within official property boundaries as indicated by airport personnel.

Habitat classifications were based on general habitat niches occupied by various wildlife taxa and for potential to act as wildlife attractants. Habitat classifications included alfalfa, bare earth/construction, cattail marsh, corn field, developed (buildings, paved roads and parking lots), dirt/gravel pile (bare or covered with weeds), ephemeral pool, fencerow, grassy swamp, gravel road, hayfield, medium grass $(10-45 \mathrm{~cm}$ tall, mowed several times per year) ornamental/shade trees, permanent water, runway system (active runways and taxiways), savanna, scrub-shrub (mix of shrubs, young trees, and tall grass), short grass ( $<10 \mathrm{~cm}$ tall, mowed weekly or bi-weekly), sorghum field, soybean field, stone swale, tall grass $(>75 \mathrm{~cm}$ tall, not mowed more than once per year), weedy ditch, wheat field, woodlot, and other (e.g., old-field habitat consisting mainly of tall grass, old runway system with short grass growing through cracked pavement). 
In addition to mapping general habitats, we carefully noted presence of wildlife attractants (e.g., standing water, refuse containers, brush piles, and culverts) during all seasons (Cleary and Dolbeer 1999). Specifically, we recorded presence of crop fields (alfalfa, corn, soybean, sorghum, wheat), woodlot refugia, standing water (permanent or ephemeral), open streams (permanent or ephemeral, flowing above-ground), open refuse containers, open buildings (e.g., hangars without closing doors), open culverts (i.e., those without grating), brush piles, and gravel piles on airport properties. With respect to open culverts, we were interested primarily in those that provided potential den sites for mammals (e.g., coyote, raccoon, skunk, or opossum) or a means of access to fenced airfields. Occasionally, we also searched surrounding properties for habitat features that might attract hazardous wildlife (e.g., potential roost sites for vultures).

Finally, we made a general assessment of the effectiveness of fencing at each airport based on fence type, proportion of airport perimeter fenced, and number and type of fence openings present. Endpoints for each fence type were documented using a handheld GPS unit. We classified 10 types of fencing based on height and construction:

Type A: 305-cm (120-in) chain-link, 3 strands of barbed wire on top Type B: 244-cm (96-in) chain-link, 3 strands of barbed wire on top

Type C: 213 - to $244-\mathrm{cm}$ (84- to 96 -in) chain-link

Type D: $213-\mathrm{cm}$ (84-in) chain-link, 3 strands of barbed wire on top

Type E: $213-\mathrm{cm}(84-\mathrm{in})$ chain-link

Type F: 183 - to $213-\mathrm{cm}$ (72- to 84 -in) chain-link, plus $30-61 \mathrm{~cm}$ (12-24 in) buried

Type G: $183-\mathrm{cm}$ (72-in) chain-link, 3 strands of barbed wire on top

Type H: $183-\mathrm{cm}$ (72-in) chain-link

Type I: 91 - to $137-\mathrm{cm}$ (36- to 54 -in) chain-link

Type J: Other: $213-\mathrm{cm}$ (84-in) plastic mesh (5-cm [2-in] squares); 183-cm (72-in) wood-panel; 91- to 137-cm (36- to 54-in) wire mesh (15-cm [6-in] squares); 5 strands barbed wire (137 cm [54 in] tall) 
For each airport with chain-link fencing (Types A-I) around $>25 \%$ of the airport perimeter, we used a handheld GPS unit to document fence openings that could be exploited by wildlife for entry onto the airfield. Where access was granted, we documented all fence openings $\geq 7.6 \mathrm{~cm}$ ( $3.0 \mathrm{in}$ ), based on our assumption that such openings would allow animals opossum-sized and larger to pass through. Generally, we did not document openings in or under wire-mesh (Type $J$ ) fences because the mesh size itself was large enough to allow passage by animals. For all other fence types, we classified seven types of openings:

Break: Opening of $<20$ meters between two segments of a fenceline (e.g., where a driveway or pedestrian corridor occurred); Sections where the fenceline is broken by $>20$ meters were considered to be unfenced portions of the airfield.

Culvert: Open culvert underneath fence

Dig-hole: Hole excavated underneath fence

Gap: Open space between bottom of fence and the ground, or between doors of a gate in the fenceline

Hole: Missing portion of a fence created by chewing/gnawing or other destructive action

Warp: Open space between bottom of fence and the ground, caused by warping or other physical damage to bottom of fence

Other: Actions outside the fenceline that have essentially eliminated effectiveness of the fence in preventing larger mammals from jumping over it (e.g, by raising the height of a road or filling a ditch with gravel)

\section{GIS within Airport Boundaries}

After we recorded presence and location of habitat patches, fence types, and fence openings at each airport, we used ArcMap (ArcGIS 9) to create digital maps of the features. First, we downloaded geographic raster data of Indiana counties where study airports were located (generated in 2003 by the USDA NAIP, provided by the IGS [http://129.79.145.5/arcims/statewide/index.html]). The sid. file for each raster download was added to an ArcMap project and served as a base map for digitizing habitat types. 
We created an individual feature class for each habitat type and fenceline, and then digitized features (including the airport property as a whole) based on data we recorded on our field maps. We attempted to achieve a minimum of 5-m accuracy for all features. Locations of fence openings were downloaded from our GPS unit and uploaded onto our digital maps.

Following completion of the maps, we used the Calculate Area tool in ArcMap to determine the area $\left(\mathrm{m}^{2}\right)$ of each polygon. Polygon areas were summed for each habitat type and converted to a percentage of the total airport area. Additionally, we calculated the perimeter of each airport, length of each fence type, proportion of airport perimeter for each fence type, and number of openings per $100 \mathrm{~m}$ of fence for each airport.

\section{GIS Outside Airport Boundaries}

We used ArcMap (ArcGIS 9) to create digital habitat maps for the area within a 10-km radius of the DeKalb County, Warsaw Municipal, and South Bend Regional airports. The 10-km radius was based on a center point established at the midpoint of the horizontal and vertical axes of the airport boundary. For the Warsaw and South Bend airports (which had completely fenced airfields), we used the fenceline as the airport boundary. For the DeKalb airport (which had little fencing or other obvious boundary markers), we used an official property boundary provided to us by airport personnel. A northern portion of the 10-km radius around South Bend Regional Airport extended into the state of Michigan; we mapped habitat only within the state of Indiana.

We first downloaded aerial imagery of counties where the three airports were located (raster data generated in 2003 by the USDA NAIP, provided by the IGS [http://129.79.145.5/arcims/statewide/index.html]). The sid. file for each raster download 
was added to an ArcMap project and served as a base map for digitizing airport boundaries and habitat types. We created individual feature classes for each habitat type and then digitized polygon features based on our interpretation of landscape features visible on the aerial imagery. We attempted to achieve a minimum of 50-m accuracy for all habitat types. Following completion of digitizing habitat types, we used the Calculate Area tool in ArcMap to determine the area $\left(\mathrm{m}^{2}\right)$ of each habitat polygon. Polygon areas were summed for each habitat type and converted to a percentage of the total area within the 10-km radius, minus the area of the airport.

We created 13 habitat classifications based on general habitat niches occupied by various wildlife taxa, potential for certain habitats to act as wildlife attractants, and ability to differentiate habitat types via aerial photo interpretation. Habitat classifications consisted of barren, forest/woods, grassland, heavy development, moderate development, open wetland, pasture, pond/river, recreational field, agriculture, shrubland, wooded wetland, and other. We defined barren habitat as any area appearing to consist of bare soil or rock (e.g., gravel pits). Forest/woods habitat consisted of wooded areas having a closed canopy and lacking any visual evidence of standing water. Grassland habitat was defined as grassy habitat that did not appear to be pasture, lawn, recreational field, or wetland; CREP fields were included in grassland habitat. Heavy development consisted of relatively large, contiguous expanses of buildings and paved surfaces that appeared to have few or no biotic features. Moderate development consisted of developed areas that contained biotic features and, therefore, had some potential as wildlife habitat (e.g., residential areas containing trees and grass, highways with grassy margins or medians). Open wetland was defined as wetland habitat having open canopy and an abundance of 
herbaceous vegetation (e.g., wet meadows, marshes, ponds with emergent vegetation). Pasture, although difficult at times to differentiate from grassland habitat, consisted of grassy areas with obvious signs of use by cattle (e.g., much visible soil, presence of small ponds, mud holes, or scattered shade trees, adjacency to farmyard). We defined pond/river habitat as permanent bodies of water having obvious boundaries and little or no emergent vegetation. Recreational fields included golf courses, baseball diamonds, school fields, and other tree-less green space in parks or campuses. Agriculture was defined as fields consisting of corn, sorghum, soybean, or other crops (we were unable to identify particular crop types); crop fields were distinguished from other green habitats (e.g., grassland, pasture) by combinations of color, area, visual evidence of plowing, and uniformity of vegetation height. Shrubland habitat was defined as non-wetland habitat consisting of a mix of grass, shrubs, and young trees. Wooded wetland habitat consisted of wetlands with an appreciable number of overstory trees (or extensive shrub layer), but having some breaks in the canopy or other obvious differentiation from upland forest (e.g., tree height). We used GIS data from the National Wetland Inventory (1971-1992; provided by the IGS [http://129.79.145.5/arcims/statewide/index.html]) to help identify open and wooded wetland habitats when presence of water was not obvious on aerial imagery. Finally, other habitat was defined as any area that we could not identify on aerial imagery or that did not clearly fit into one of the other 12 habitat classifications; examples included composting sites, cemeteries, and municipal parks having a fair number of trees.

\section{Pilot Questionnaires}


We sent 50 copies of a questionnaire (Appendix) to the operating manager of each airport, who agreed to distribute the questionnaires among the airport's most regular pilots. Questionnaires were sent with addressed, stamped envelopes and returned individually via mail between 1 October 2005 and 1 January 2006. The questionnaire was designed to assess the opinions and experiences (with respect to wildlife hazards) of pilots who regularly used at least one of the 10 participating airports.

We note that readers should exercise caution when interpreting results of volunteer, mail-only surveys such as ours. Potential inferences are limited because we cannot guarantee that the respondents represented a random sample of pilots who used the participating airports. For example, pilots who generally did not encounter wildlife during their flights may have been less likely to return a questionnaire than those who felt that wildlife hazards were especially problematic. However, our primary goals for the questionnaire were to gain a preliminary understanding of 1) pilot attitudes concerning wildlife hazards at Indiana airports and 2) opinions regarding management techniques.

\section{$\underline{\text { FAA Strike Database }}$}

The Sandusky, Ohio Field Station of the USDA/APHIS/WS/National Wildlife Research Center maintains the National Wildlife Strike Database, which catalogs all reported wildlife strikes at airports in the U.S. For each wildlife strike, the database contains information on location, timing, species struck, weather conditions, altitude and speed of aircraft at impact, and damage to aircraft for the period of 1990-present. We requested all strike data from participating airports on 1 October 2005 from the database manager at Sandusky. Here, we report a simplified version of the strike data for each of the participating airports. We note, however, that only about $20 \%$ of all wildlife strikes are 
reported (Cleary et al. 2003). Thus, the wildlife strikes depicted here are certainly an underestimate of the true number of strikes at our study airports.

\section{RESULTS}

\section{$\underline{\text { Wildlife Inventories }}$}

\section{Spotlight Surveys}

The number of hazardous species observed during spotlight surveys varied greatly among airports (Table 2). We observed up to 50 deer (across all surveys; up to 20 on any individual survey) and 9 coyotes during our surveys. Only three airports had no deer inside the property boundaries during spotlight surveys, whereas six airports had no coyotes (Table 2). Encounters of smaller mammals also varied greatly among airport properties (Table 2).

\section{Bird Surveys}

Of the 16 species groups identified by Dolbeer et al. (2000) as hazardous to aircraft (Table 3), American kestrel, blackbirds-starling, crows-ravens, mourning dove, shorebirds, sparrows, and swallows were present at 9-10 of the airport properties at some point during the year. Geese, hawks (buteos), and vultures were present at 7-8 of the airport properties; and ducks, herons, and rock doves were present at 5-6. Gulls, eagles, and cranes each were present at only 1 airport property. Bird abundances also varied widely depending on species and location. Among all airports, the most numerous species group was blackbirds-starling (including red-winged blackbirds, eastern meadowlarks, brown-headed cowbirds, common grackles, and European starlings), although the totals were skewed somewhat by a flock of blackbirds (over 2000 
individuals) that was observed during one of the fall surveys at DeKalb Co. Airport (Table 3).

\section{Remote Camera Surveys}

At least 26 species were observed on airport properties with remote cameras (we were unable to identify 25 individuals observed on camera) (Table 4; Plates. 1-5). Coyotes were observed at seven airports (the highest number of observations at an individual airport was 15), and deer were observed at six (the highest number of observations at an individual airport was 39). Due to increased battery life in winter, cameras were used for $30,159 \mathrm{hr}$ in winter (totaled across all airports), which was much more than during any other season (at most, 9,394 hr). As a result, we surveyed more individual animals during the winter season (254 observations across all species; 0.00842 animals per camera-hr) than during other seasons (226 observations for spring, summer, and fall combined; 0.00818 animals per c-hr). In particular, during winter we observed more deer (92 observations in winter; 50 observations across the other three seasons combined) and coyotes (35 observations in winter; 5 observations across the other three seasons combined) via camera.

\section{Opportunistic Observations}

Twenty-one observations of coyotes (across eight airports), 30 of deer (across four airports), 32 of red-tailed hawks (across six airports), 34 of turkey vultures (across six airports) and 36 of Canada geese (across five airports) were tallied opportunistically during other research activities.

\section{Habitat and Fencing Evaluation}




\section{On-Site Assessments and GIS within Airport Boundaries}

Habitat types present at all airport properties were runway systems, other developed areas, and short grass (Table 5; Figs. 11-30). The next most commonly occurring habitats were scrub-shrub ( $\mathrm{n}=8$ airports), tall grass $(\mathrm{n}=8)$, weedy ditches $(\mathrm{n}=8)$, medium grass $(n=7)$, bare earth/construction $(n=6)$, and soybean fields $(n=6)$. Corn fields, dirt/gravel piles, ephemeral pools, gravel roads, permanent water, and woodlots each were present at five of the airports (Table 5). On average, airport habitats consisted mainly of short grass $(40.2 \%$ of total airport area), soybean fields $(10.3 \%)$, corn fields (9.5\%), runway systems $(8.1 \%)$, other development (6.6\%), woodlots $(5.2 \%)$, medium grass $(4.8 \%)$, tall grass $(4.6 \%)$, and hayfields $(3.2 \%)$; however, averages for each of the hayfield and tall grass habitats were skewed by a large value at one airport (Table 5). All

other habitat types averaged $\leq 1.3 \%$ of total airport area, but alfalfa and sorghum fields each represented $\sim 10 \%$ at airports where they occurred.

At least two types of wildlife attractants were present on each airport property, but most airports had five to seven types (Table 6). The most common attractants were standing water (ephemeral) and open culverts, which were present at eight airports each (Table 6). Other common attractants were crop fields, woodlot refugia, and gravel piles. Attractants adjacent to airports included a state park (primarily wooded) next to Anderson Municipal, a golf course next to Clark County, a woodlot that we suspect is the site of a vulture roost next to DeKalb County, ponds in commercial lots next to Greenwood Municipal, ponds and feeding operations in pastures next to Huntingburg, a major river (Wabash River) and ponds near Purdue University, a pond and park next to Putnam County, a composting operation and golf course next to South Bend Regional, and a pond 
within a residential neighborhood next to Warsaw. Crop fields were adjacent to almost all airports. Of particular concern were circumstances at several airports (Anderson Municipal, Clark County, Huntingburg, and Putnam County) where runway systems occurred between sources of shelter (e.g., woodlot refugia) and sources of food (e.g., cropfields, streams) for wildlife.

Each airport used Type G, H, I, or J fencing along at least a portion of its perimeter, and seven airports used only those types (Table 7; Figs. 31-40). Proportion of airport perimeter fenced ranged from $7.5 \%$ to $100 \%$ among all airports, but most perimeters were $>40 \%$ fenced (Table 7). Only four airports (Greenwood Municipal, Purdue University, South Bend Regional, and Warsaw Municipal) were completely fenced; however, all four had openings in their fencelines that would allow coyotes and, perhaps, deer access to the airfields (Table 8; Figs. 31-40). Most airports having $>25 \%$ of the perimeter fenced with chain-link fencing had $0.2-0.5$ openings per $100 \mathrm{~m}$ of fence, with gaps and dig-holes being the most common openings (Table 8). Purdue University Airport had 1.3 openings per $100 \mathrm{~m}$ of fence, due largely to a segment of plastic-mesh fencing with 59 holes chewed through it.

\section{GIS Outside Airport Boundaries}

Landscapes within a 10-km radius of DeKalb County, South Bend Regional, and Warsaw Municipal airports averaged approximately $40 \%$ agriculture, $22 \%$ moderate development, $15 \%$ forest $/$ woods, and $7 \%$ grassland habitat; all other habitat types represented $\leq 4 \%$ of the landscape (Table 9; Figs. 41-46). With the exception of a relatively large patch of forest/woods and a large patch of moderate development, habitat types appeared to be evenly distributed across the landscape surrounding DeKalb County Airport (Figs. 41- 
42). However, some habitat types appeared to be more clustered around the South Bend Regional (Figs. 43-44) and Warsaw Municipal airports (Figs. 45-46). At South Bend, large patches of development and recreational fields, which harbor wildlife attractants like fast-food restaurants and trash receptacles, dominated the landscape south and east of the airport. The remaining landscape consisted primarily of agriculture. At Warsaw, development was clustered south of the airport and agriculture was abundant to the east, north, and west. Of important note is that relatively large ponds occurred within the 10km radius surrounding Warsaw Municipal Airport (Fig. 45), and they amounted to 2.84.7 times the proportion of ponds/rivers in landscapes surrounding DeKalb County and South Bend Regional airports.

\section{Pilot Questionnaires}

Of 500 surveys distributed among airport managers, 84 (16.8\%) were returned. The cover sheet, questionnaire, and a tally of responses are presented in the Appendix. Respondents largely were experiences pilots $-55 \%$ reported over 20 years experience operating aircraft (Question 1). The level of participation in the survey was mixed among airports (e.g., 18 respondents indicated that Purdue University Airport was their primary airport, whereas only 2 indicated that Greenwood Municipal Airport was their primary airport; Question 3). Fifty-eight of 84 (69\%) respondents reported that they have had to alter flight, landing, or take-off plans because of wildlife occurring within air operations areas at least once during the past year (Question 5), but only 21 of $84(25 \%)$ reported that they had been involved in a wildlife collision during the past year (Question 6). Forty-nine of $83(59 \%)$ respondents felt that their primary airport needed some level of improvement in terms of wildlife management, whereas $34(41 \%)$ respondents felt that 
improvement was not needed, or had no opinion (Question 7). Sixty-five of 84 respondents $(77 \%)$ had been in at least one wildlife collision during their entire course of experience as a pilot (Question 8); small birds and geese were struck most often (Question 9).

Only three respondents reported that wildlife hazards had caused them to permanently cease operating aircraft at a particular airport (Question 10). However, 36 of 81 respondents (44\%) felt that wildlife populations at airports in Indiana were either hazardous or very hazardous in terms of their potential for aircraft collisions (Question 11). An additional 36 respondents (44\%) reported "somewhat hazardous" for the same question; thus, only 9 respondents (11\%) felt that wildlife were not hazardous at Indiana airports. Although most respondents felt that wildlife on Indiana airports were problematic, they did not appear to accept potential management methods equally (Question 12). Fifty-six of 81 respondents (69\%) supported or strongly supported construction of exclosures (fencing), and 55 of 82 respondents (67\%) supported or strongly supported use of wildlife deterrents. However, only 35 of 82 respondents (43\%) indicated support for modification/elimination of wildlife habitat, and only 31 of 82 respondents (38\%) indicated support for direct removal of wildlife.

\section{FAA Strike Database}

Seventy-four wildlife strikes were reported from South Bend Regional Airport and 10 from Purdue University Airport (Table 10 [electronic only]). No other airport reported more than five strikes. Thirty-nine of the entries reported strikes with "unknown birds", nine with hawks (unknown hawks or red-tailed hawks), eight with gulls, seven with sparrows, and six with European starlings. Seven mammals were reported as being 
struck by aircraft — five white-tailed deer, one skunk, and one coyote. Total losses were $\$ 2,520,522$ (repairs and other costs) and 4690 hours. Eight entries reported an aborted take-off, four reported a precautionary landing, and one reported an engine shut-down.

\section{DISCUSSION}

Our research demonstrated that despite the lack of published information concerning wildlife hazards at small airports, the potential for significant wildlife strikes at such sites in Indiana does exist. Our habitat assessments, wildlife surveys, and pilot questionnaires all indicated that more emphasis should be given to the problem of wildlife strikes by airport personnel at general aviation airports in Indiana.

\section{$\underline{\text { Wildlife Inventories }}$}

Our observations from spotlight surveys, remote camera surveys, and opportunistic sightings indicated that deer and coyotes are common visitors to air operations areas of several focal airports. Across seasons and surveys, the total number of deer at focal airports ranged from 0-92, and the total number of coyotes ranged from 0-28 (Table 11). Dolbeer et al. (2000) ranked deer and coyotes as the top two mammalian aviation hazards in the U.S. (number 1 and 15 overall, respectively), thus their presence in critical airport areas warrants concern. However, there was a great deal of variation in the number of deer and coyotes surveyed across airports. Purdue University Airport was the only focal airport where we observed no deer or coyotes, and interestingly, it has a stringent wildlife hazard management program (Betty Stansbury, personal communication). Furthermore, completely-fenced airports (Greenwood, Purdue, South Bend, Warsaw) appeared to have fewer problems with deer and coyotes (total observations; mean $=6.0$ and 2.8 , respectively) than airports that were not completely fenced (45.5 and 10.7). Although 
other species of mammals observed within airport properties (e.g., opossums, raccoons, domestic cats) rarely cause substantial damage (Cleary et al. 2005), their presence is noteworthy and should be considered a hazard because they are struck regularly by aircraft.

Results of our bird surveys also indicated that many hazardous species are present regularly at Indiana airports. Of the 19 species and species groups identified by Dolbeer et al. (2000) as most hazardous to aircraft, 16 were present (and in many cases, abundant) at Indiana airports. Only osprey (rare in Indiana), pelicans (rare in Indiana), and owls (nocturnal and thus not active during our surveys) were not observed. Furthermore, the two most hazardous species groups, vultures and geese, were observed at 7 and 8 airports, respectively. Certainly, many airport environments are attractive to a variety of bird species for roosting, nesting, feeding, and loafing.

\section{$\underline{\text { Habitat and Fencing Evaluation }}$}

Habitat and fencing regimes likely explain hazards posed by mammals at the airports we studied. Deer appeared to be abundant either within or just outside all airport properties, except Greenwood Municipal and Richmond Municipal. Woodlots and shrublands are preferred habitats for deer and other mammals with respect to shelter and food, and a lack of both habitats on or near Greenwood Municipal and Richmond Municipal airports may explain why we rarely observed deer at those locations. Although much of Richmond Municipal is crop-field habitat and, therefore, a potential feeding area for deer, other crop fields immediately surround the airport and may act as a buffer by satiating deer associated with remote woodlots outside the property. Likewise, Greenwood Municipal is bordered by development to the east and crop fields to the west, thereby providing little 
incentive for deer inhabiting remote woodlots to cross those habitat patches to feed on crops within the fenceline. Woodlot or shrubland habitats were present in or adjacent to all other airports, thus fencing was the only barrier preventing deer, coyote, raccoons, and opossums from accessing airfields. However, based on our analyses of fence openings, none of the focal airports had a fencing regime adequate to exclude all individuals from the airfields. For example, Greenwood Municipal, Purdue University, and South Bend Regional airports had completely fenced airfields, but all fences were seriously flawed. The fence at Greenwood had a 5-m break and several large ( $>1 \mathrm{~m})$ gaps where a stream entered and exited the property; the stream attracted raccoons (as many as 5 individuals observed together at one time) and the fence openings allowed access to the airfield. Although we did not observe deer, coyotes, or raccoons within the fenceline at Purdue, a portion of the fenceline consisted of plastic mesh and contained numerous holes that had been chewed by animals. These holes, and others dug by coyotes, certainly provided access to the airfield; in fact, coyotes were observed within the fenceline several times by airport personnel. At South Bend, numerous dig-holes allowed opossums, raccoons, coyotes, and perhaps deer to cross under the fenceline.

Although no fencing regimes were completely mammal-proof, our results suggested that chain-link fencing around the entire perimeter of an airport was effective in minimizing abundance of mammals on airfields. We tended to observe mammals within airport properties much more frequently at airports with incomplete fencing than at Greenwood Municipal, Purdue University, South Bend Regional, or Warsaw Municipal airports, suggesting that animals were less likely to use an airfield if access to it required passage through a fence opening. 
Presence of red-tailed hawks and American kestrels appeared to be linked strongly to habitats occurring within and immediately adjacent to the airports we studied. Almost all airports had a resident pair of red-tailed hawks that were observed perching on weather stations, light towers, or other objects on airfields. American kestrels also were observed hunting along taxiways and perching on runway lights at most airports. Notable exceptions, however, were Putnam County and Richmond Municipal airports, where both species were relatively uncommon. We suspect that the presence of hawks and kestrels was dependent on availability of grassland habitat (hayfields and short/medium/tall grass) and, perhaps, perches. Grassy areas provide habitat for rabbits, ground squirrels, voles, and other small mammals that constitute major food items for hawks and kestrels, and perches serve as resting areas and hunting platforms. Perches were rare at Richmond, and grassland habitat (short, medium, or tall) represented a much lower percentage (9\%) of total airport area than at other airports (28-82\%). Although perches and grassland habitat were readily available at Putnam County, voles and other rodents may have been rare because the airfield had undergone major reconstruction and re-seeding just prior to our study.

Geese, ducks, and herons typically are associated with wetland habitats; however, large expanses of short grass also may attract geese. We did not observe any of these groups of birds at Richmond Municipal, probably because ponds and rivers were absent from the airport property and rare in the surrounding landscape. Geese, ducks, or herons were observed at all other airports except Anderson Municipal. Presence of geese and ducks at Greenwood Municipal, Putnam County, South Bend Regional, and Warsaw Municipal airports was clearly linked to occurrence of ponds on or adjacent to airport 
properties. Herons were most notable at DeKalb County, Purdue University, and Warsaw Municipal airports, where large bodies of water in surrounding landscapes provided ample habitat. Individual herons were observed in a permanent stream at Greenwood Municipal and at an ephemeral pool (where chorus frogs were breeding) at Clark County. For the most part, geese tended to occur in flocks and used short-grass habitat on airfields only temporarily (several days to 2 weeks, according to reports from some airport managers). These flocks tended to occur during late summer and fall and, therefore, probably constituted migratory groups using airfields as stopover habitat. However, we did note resident pairs at Putnam County and South Bend Regional airports, where small ponds were present either on or adjacent to airfields. In fact, two pairs of geese nested within the airport boundary at South Bend. Of particular concern was a pair of geese at Putnam County that frequently loafed on the runway during the spring.

Crows and starlings often exhibit flocking behavior, which can pose a serious hazard to aviation. Moreover, populations of crows and starlings often are associated with human development and urban areas because they are sources of food or loafing areas (e.g., fast food restaurants, large trash receptacles, recreational fields, powerlines, etc.). Most of the airports we studied consisted of $\geq 10 \%$ developed habitat (including runway systems) and occurred within several kilometers of urban areas. We observed large ( $>50$ individuals) flocks of crows at Anderson Municipal, Richmond Municipal, and South Bend Regional airports. Large flocks of starlings occurred at all airports except Putnam County, but they appeared to be most hazardous at Anderson Municipal, Greenwood Municipal, Huntingburg, Purdue University, and South Bend Regional airports, based on flock sizes and tendency to fly over runways. We observed starlings 
on multiple occasions repeatedly flying over the runway at Greenwood Municipal and returning to open hangars with nesting material. Also of note were flocks of starlings and cowbirds that gathered at cattle-feed stations in a pasture next to Huntingburg airport. These flocks were especially hazardous because they moved frequently and without warning when they were startled by hawks overhead or by cattle in the pasture.

\section{$\underline{\text { Pilot Questionnaires }}$}

Data from the questionnaire clearly indicated that pilots using focal airports were familiar with wildlife hazards. Sixty-nine percent of respondents reported that they had altered aircraft operation due to wildlife hazards within the past year, and 25\% reported involvement in a wildlife strike during the past year. Furthermore, $88 \%$ of respondents felt that wildlife populations at Indiana airports were at least "somewhat hazardous". Because of the high level of exposure to wildlife hazards experienced by respondents, we were surprised at the relatively low level of support for wildlife hazard management expressed by respondents. Although $70 \%$ of respondents supported the use of fencing or wildlife deterrents, only $43 \%$ supported modification/elimination of wildlife habitat, and merely $38 \%$ of respondents supported lethal removal of wildlife. We suspect that the relatively low level of support for wildlife hazard management at airports stemmed from the fact that wildlife were observed on airport properties without incident much more often that actual wildlife strikes occurred. This may have led respondents to believe that wildlife species are not problematic on airport properties because they "usually get out of the way". However, pilots and other airport personnel should be aware of the seriousness of the wildlife hazard problem at airports.

\section{FAA Strike Database}


There were relatively many wildlife strikes records in the FAA database from South Bend and Purdue compared to the other focal airports. However, we suspect that the number of strikes reported to the FAA from various airports may better reflect the willingness of airport personnel to report strikes than the actual number of strikes that occurred at each airport. Universally, only about $20 \%$ of all wildlife strikes are thought to be reported to the FAA (USDA/APHIS 2004). Certainly, all airport personnel should be encouraged to report all wildlife strikes to the FAA on form 5200-7 (http://wildlifemitigation.tc.faa.gov).

\section{MANAGEMENT RECOMMENDATIONS}

The science of wildlife damage management is growing and prospering (Conover 2002); new technologies and refinements in techniques are emerging continuously. Many new methods are especially relevant to managing hazardous wildlife on airport properties. For example, a new, relatively inexpensive brand of electric fence (Electrobraid ${ }^{\mathrm{TM}}$; Yarmouth, Nova Scotia, Canada) has shown promise in excluding free-ranging deer (Seamans and VerCauteren 2006), and a new motion-activated laser hazing system that was evaluated recently may prove useful for dispersing Canada geese from airports and other areas where they are not desirable (Werner and Clark 2006). Effigies of dead conspecifics recently have shown to be effective in dispersing vultures (Avery et al. 2002, Seamans 2004). However, despite recent progress in developing new methods, we stress that it is imperative airport personnel be familiar with established techniques in wildlife hazard management at airports, such as those summarized in Cleary and Dolbeer (1999). Most wildlife hazard problems facing airport managers can be addressed with traditional methods, such as reducing wildlife cover and removing standing water. Here, 
we focus on new technologies and specific management recommendations that might benefit our focal airports.

Deer and coyotes represent the most worrisome mammalian hazards at airports. Exclusion is the preferred method (and in many cases, the only effective method) of preventing deer and coyotes from using large areas (Conover 2002) and can be accomplished with proper fencing. Researchers have demonstrated that a $2.4-\mathrm{m}$ fence (8 ft) usually can exclude non-stressed deer on level ground (Falk et al. 1978). However, because motivated deer can clear a 2.4-m fence, 3-m (10 ft) fencing may be more appropriate in airport environments where complete exclusion is desired (VerCauteren et al. 2006). Despite the effectiveness and durability of fences, installation is expensive (e.g., 2.4-m chain link fencing costs $>\$ 20 / \mathrm{m}$; VerCauteren et al. 2006). At airports where complete exclosure with tall fencing is prohibitively expensive, we suggest airport managers consider newer alternatives, such as Electrobraid ${ }^{\mathrm{TM}}$ (Seamans and VerCauteren 2006). Electrobraid ${ }^{\mathrm{TM}}$ has not yet been evaluated experimentally in an airport environment, but initial experiments on smaller areas appear promising (Seamans and VerCauteren 2006).

Although very tall (i.e., 3-m) chain-link or woven-wire fences are ideal in airport settings, we propose that a shorter, well maintained 2.4-m fence may be more effective in excluding deer and coyotes than a 3-m fence with an abundance of gaps and holes. A 25cm gap at the bottom of a fence can allow an adult deer to get through (Falk et al. 1978, Palmer et al. 1985, Feldhamer et al. 1986), and when properly motivated, adult deer can pass through a 19-cm gap (Feldhamer et al. 1986). Many of the focal airports in the present study had fences with an abundance of gaps that could allow passage for deer and 
coyotes. In many respects, a fence is only as effective as its weakest section. We advocate regular fence maintenance and immediate repair of damaged fences. Many problems associated with fence gaps can be eliminated by using a buried-fence design. We strongly recommend that buried fences are considered when installing new airport fences.

We stress that there should be no tolerance for deer or coyotes inside airport fences (Cleary and Dolbeer 1999). At several focal airports we observed that personnel did not seem overly bothered by deer and coyotes near runways, because the animals usually did not cause problems (this attitude also was reflected in pilot surveys). Individual animals that have managed to breach wildlife fencing and occupy air operations areas should be removed (by lethal means if necessary) as soon as possible with appropriate authorization. Deer are intelligent and learn from observing others; thus, as individual deer learn to penetrate fences, continued effectiveness of the barrier depends on prompt removal of those individuals (VerCauteren et al. 2006).

Birds often present more challenging management problems than mammals because they are not deterred by fencing. Thus, to manage hazardous birds effectively in airport environments, one usually must rely on habitat management or fear-provoking stimuli (Conover 2002). Fear provoking stimuli (frightening devices such as propane cannons and scarecrows) can successfully repel birds from critical areas; however, their effects usually are temporary (Conover 2002). Birds quickly become accustomed to frightening devices and learn to ignore them. One possible exception, however, is the use of dead conspecifics or effigies of dead conspecifics to repel some bird species. Research on vultures (Avery et al. 2002, Seamans 2004) and gulls (Stout et al. 1975, Stout and 
Schwab 1979) has demonstrated that such species are repelled by effigies of dead conspecifics or actual dead conspecifics in several settings. Similar preliminary research with Canada geese has produced mixed results (Seamans and Bernhardt 2004). Importantly, the repelling effect of such stimuli for vultures and gulls appears to be more permanent than other frightening devices. Airports with persistent vulture or gull hazards should investigate the use of dead bird effigies to repel unwanted birds. This emerging method may be especially useful for repelling vultures at nocturnal roosts. Vultures, which are increasing in number throughout much of the U.S. (Avery 2004), usually do not range far from nocturnal roosts (DeVault et al. 2004). Dispersal from nocturnal roosts on or near airport properties should greatly decrease the number of vultures present near critical areas.

Although dead bird effigies may reduce hazards associated with some species, habitat management may provide a more permanent solution to bird hazards at airports in general. The overall goal of habitat management at airports is to reduce the availability and/or quality of food, water, cover, and loafing sites for hazardous species. As such, most biologists agree that wetlands, standing water, cereal grains, open trash receptacles, and woodlots should not be present in airport environments (Cleary and Dolbeer 1999). Also, hangars and other buildings should be kept closed, and all openings in buildings should be plugged with wood or sheet metal to prevent access to eaves or other nesting platforms by European starlings. We also note that large flocks of mourning doves and killdeer could be reduced by paving gravel roadways and parking areas, and by minimizing the amount of bare earth present on property by planting grass or paving. 
Many small airports in the Midwest (including several focal airports in the current study) produce crops on airport properties to increase revenue. Crops such as corn and soybeans attract wildlife and ideally should not be present near air operations areas. However, where agricultural operations are deemed necessary on airport properties, postharvest crop residue should be plowed under to minimize attractiveness to wildlife during the non-growing season (Cleary and Dolbeer 1999).

Although some habitat management practices are well accepted and considered standard operating procedure, there is no clear consensus concerning vegetation management (turf grass management in particular), because no single vegetation type is unattractive to all species (Barras and Seamans 2002). For example, biologists disagree about the proper grass height in airport infields. Airports commonly maintain grass height from 5-45 cm, and few data are available to support recommendations of various strategies (Barras and Seamans 2002). Tall vegetation may repel some birds due to decreased visibility, feeding activity, and ground movements, but may attract some ground-nesting species. Furthermore, tall grass supports large populations of prey including insects and rodents, which attract raptors. Conversely, short vegetation may provide loafing and feeding areas for gulls and some insectivorous birds (Barras and Seamans 2002), but discourages nesting by blackbirds, meadowlarks, and some sparrows. We suggest that individual airport managers assess their respective properties and choose the most appropriate vegetation in consultation with a qualified wildlife biologist.

One promising emerging technique in vegetation management at airports is the planting of tall fescue (Festuca arundinacea), a bunch grass that commonly is infected with the fungus Neotyphodium coenophialum (Clay and Holah 1999, Barras and Seamans 
2002). The fungus acts as a repellant to herbivores such as Canada geese and small mammals (which attract raptors). Current studies are underway to determine the efficacy of planting and propagating tall fescue in airport environments.

\section{REFERENCES}

Arrington, D. P. 2003. Flight characteristics of non-migrating and migrating populations of Turkey Vultures. Dissertation, Purdue University, West Lafayette, Indiana.

Avery, M. L. 2004. Trends in North American vulture populations. Proceedings of the Vertebrate Pest Conference 21:116-121.

Avery, M. L., J. S. Humphrey, E. A. Tillman, K. O. Phares, and J. E. Hatcher. 2002. Dispersing vulture roosts on communication towers. Journal of Raptor Research $36: 45-50$.

Barras, S. C., and T. W. Seamans. 2002. Habitat management approaches for reducing wildlife use of airfields. Proceedings of the Vertebrate Pest Conference 20:309315.

Bibby, C. J., N. D. Burgess, and D. A. Hill. 1992. Bird census techniques. Academic Press, San Diego, California.

Bookhout, T. A., Editor. 1996. Research and management techniques for wildlife and habitats. Fifth ed., rev. The Wildlife Society, Bethesda, Maryland.

Clay, K., and J. Holah. 1999. Fungal endophyte symbiosis and plant diversity in successional fields. Science 285:1742-1744.

Cleary, E. C. and R. A. Dolbeer. 1999. Wildlife hazard management at airports. Federal Aviation Administration, Office of Airport Safety and Standards, Airport Safety and Compliance Branch, Washington, DC. 
Cleary E. C., S. E. Wright, and R. A. Dolbeer. 1999. Wildlife strikes to civil aircraft in the United States, 1990-1998. Wildlife Aircraft Strike Database, Serial Report 5, Federal Aviation Administration, Office of Airport Safety and Standards, Washington, DC.

Cleary E. C., R. A. Dolbeer, and S. E. Wright. 2003. Wildlife strikes to civil aircraft in the United States, 1990-2002. Wildlife Aircraft Strike Database, Serial Report 9, Federal Aviation Administration, Office of Airport Safety and Standards, Washington, DC.

Cleary E. C., R. A. Dolbeer, and S. E. Wright. 2005. Wildlife strikes to civil aircraft in the United States, 1990-2004. Wildlife Aircraft Strike Database, Serial Report 11, Federal Aviation Administration, Office of Airport Safety and Standards, Washington, DC.

Cleary E. C., R. A. Dolbeer, and S. E. Wright. 2006. Wildlife strikes to civil aircraft in the United States, 1990-2005. Wildlife Aircraft Strike Database, Serial Report 12, Federal Aviation Administration, Office of Airport Safety and Standards, Washington, DC.

Conover, M. R. 2002. Resolving human-wildlife conflicts: the science of wildlife damage management. CRC Press, Boca Raton, Florida.

DeVault, T. L., B. D. Reinhart, I. L. Brisbin Jr., and O. E. Rhodes Jr. 2004. Home ranges of sympatric black and turkey vultures in South Carolina. Condor 106:706-711.

DeVault, T. L., B. D. Reinhart, I. L. Brisbin Jr., and O. E. Rhodes Jr. 2005. Flight behavior of black and turkey vultures: implications for reducing bird-aircraft collisions. Journal of Wildlife Management 69:601-608. 
Dolbeer, R. A., J. L. Belant, and J. L. Sillings. 1993. Shooting gulls reduces strikes with aircraft at John F. Kennedy International Airport. Wildlife Society Bulletin $21: 442-450$.

Dolbeer, R. A., S. E. Wright, and E. C. Cleary. 2000. Ranking the hazard level of wildlife species to aviation. Wildlife Society Bulletin 28:372-378.

Falk, N. W., H. B. Graves, and E. D. Bellis. 1978. Highway right-of-way fences as deer deterrents. Journal of Wildlife Management 42:646-650.

Feldhamer, G. A., J. E. Gates, D. M. Harman, A. J. Loranger, and K. R. Dixon. 1986. Effects of interstate highway fencing on white-tailed deer activity. Journal of Wildlife Management 50:497-503.

Kershner, E. L., and E. K. Bollinger. 1996. Reproductive success of grassland birds at east-central Illinois airports. American Midland Naturalist 136:358-366.

Lovell, C. D. 1997. The United States Air Force bird avoidance model for low-level training flights. Pages 162-170 in C. D. Lee and S. E. Hygnstrom, editors. 13th Great Plains Wildlife Damage Control Workshop Proceedings. 16-19 April, Nebraska City, Nebraska.

Palmer, W. L., J. M. Payne, R. G. Wingard, and J. L. George. 1985. A practical fence to reduce deer damage. Wildlife Society Bulletin 13:240-245.

Seamans, T. W. 2004. Response of roosting turkey vultures to a vulture effigy. Ohio Journal of Science 104:136-138.

Seamans, T. W., and G. E. Bernhardt. 2004. Response of Canada geese to a dead goose effigy. Proceedings of the Vertebrate Pest Conference 21:104-106. 201K 
Seamans, T. W., and K. C. VerCauteren. 2006. Evaluation of Electrobraid ${ }^{\mathrm{TM}}$ fencing as a white-tailed deer barrier. Wildlife Society Bulletin 34:8-15.

Sodhi, N. S. 2002. Competition in the air: birds versus aircraft. Auk 119:587-595.

Stout, J. F., W. H. Gillett, J. L. Hayward Jr., and C. J. Amlaner Jr. 1975. Dispersal of seagulls in an airdrome environment. Air Force Weapons Laboratory Final Report AFWL-TR-74-324, Kirtland Air Force Base, NM.

Stout, J. F., and E. R. Schwab. 1979. Behavioral control of seagulls at Langley Air Force Base. Pp. 96-110 in W. B. Jackson, S. S. Jackson, and B. A. Jackson (Eds.), Proceedings of the Eighth Bird Control Seminar, Bowling Green State University, Bowling Green, Ohio.

USDA/APHIS. 2004. Strike one-you're out! Program Aid No. 1644.

VerCauteren, K. C., M. J. Lavelle, and S. E. Hygnstrom. 2006. Fences and deer-damage management: a review of designs and efficacy. Wildlife Society Bulletin 34:191200.

Werner, S. J., and L. Clark. 2006. Effectiveness of a motion-activated laser hazing system for repelling captive Canada Geese. Wildlife Society Bulletin 34:2-7. 
Table 1. Characteristics of 10 airports chosen as study sites for an investigation of wildlife hazards at general aviation airports in Indiana in 2005-2006. Acreages are based on information provided on the INDOT website

(http://www.in.gov/dot/modetrans/airports/aerials.html).

$\begin{array}{lccccc}\text { AIRPORT } & \text { ACREAGE } & \text { RUNWAY LNG (ft) } & \begin{array}{c}\text { BASED } \\ \text { AIRCRAFT }\end{array} & \begin{array}{c}\text { SPOTLIGHT } \\ \text { TRANSECT LNG (m) }\end{array} & \begin{array}{c}\text { BIRD TRANSECT } \\ \text { LNG (m) }\end{array} \\ \text { Anderson } & 500 & 5400 & 81 & 5334 & 1133 \\ \text { Clark Co. } & 420 & 5500 & 135 & 6429 & 1478 \\ \text { DeKalb Co. } & 600 & 5000 & 56 & 2009 & 1532 \\ \text { Greenwood } & 148 & 4901 & 107 & 1644 & 1009 \\ \text { Huntingburg } & 480 & 5000 & 33 & 4720 & 1323 \\ \text { Purdue } & 500 & 6600 & 105 & 7929 & 1848 \\ \text { Putnam Co. } & 192 & 5000 & 25 & 1633 & 790 \\ \text { Richmond } & 702 & 5500 & 32 & 4941 & 1086 \\ \text { South Bend } & 1550 & 8412 & 58 & 7339 & 788 \\ \text { Warsaw } & 557 & 6000 & 49 & 2783 & 1139\end{array}$

*BASED AIRCRAFT = total number of aircraft (single engine, multi-engine, jet) permanently based at airport 
Table 2. Wildlife observed during eight spotlight surveys at each of 10 airports in Indiana in 2005-2006.

\begin{tabular}{|c|c|c|c|c|c|c|c|c|c|c|}
\hline AIRPORT & PROPERTY & DEER & $\begin{array}{l}\text { DEER } \\
\text { HIGH }\end{array}$ & COYOTE & OPOSSUM & RACCOON & SKUNK & $\begin{array}{l}\text { DOM. } \\
\text { CAT }\end{array}$ & $\begin{array}{l}\text { RED } \\
\text { FOX }\end{array}$ & RABBIT \\
\hline \multirow[t]{2}{*}{ Anderson } & \multirow{4}{*}{$\begin{array}{l}\text { Inside } \\
\text { Outside } \\
\text { Inside } \\
\text { Outside }\end{array}$} & 50 & 20 & & 2 & 8 & 1 & 1 & & 1 \\
\hline & & & & & & 3 & & & & \\
\hline \multirow[t]{2}{*}{ Clark Co. } & & 26 & 7 & 9 & & & & & 3 & 2 \\
\hline & & & & & & & & & & \\
\hline \multirow[t]{2}{*}{ DeKalb Co. } & Inside & 18 & 5 & & 1 & 4 & 2 & 1 & & 2 \\
\hline & Outside & & & & & & & & & \\
\hline \multirow[t]{2}{*}{ Greenwood } & Inside & & & & & 1 & & & & 1 \\
\hline & Outside & & & 1 & & & & & & \\
\hline \multirow[t]{2}{*}{ Huntingburg } & Inside & 4 & 4 & 2 & & 1 & & 1 & & 5 \\
\hline & Outside & 4 & 2 & & & & & & & 1 \\
\hline \multirow[t]{2}{*}{ Purdue } & Inside & & & & 1 & & & & & 2 \\
\hline & Outside & 3 & 2 & & & & & & & \\
\hline \multirow[t]{2}{*}{ Putnam Co. } & Inside & 19 & 7 & & & 4 & 1 & & 1 & \\
\hline & Outside & & & & & & & & & \\
\hline \multirow[t]{2}{*}{ Richmond } & Inside & 1 & 1 & & & 2 & 4 & 2 & & \\
\hline & Outside & & & & & & & & & \\
\hline \multirow[t]{2}{*}{ South Bend } & Inside & 7 & 4 & 2 & 4 & & & 1 & & 6 \\
\hline & Outside & 1 & 1 & & & & & & & \\
\hline \multirow[t]{2}{*}{ Warsaw } & Inside & & & 1 & 1 & 1 & 11 & 1 & & \\
\hline & Outside & & & & & & & & & \\
\hline
\end{tabular}

*PROPERTY = Inside or Outside airport property as defined by fencing or defined by airport personnel.

${ }^{*}$ DEER HIGH = high count of deer during any single survey. 
Table 3. Birds observed during eight walking transect surveys at each of 10 airports in Indiana in 2005-2006. We report only hazardous species (as defined by Dolbeer et al. 2000) observed within airport properties. For each species, the high count for each season (of two counts) is reported.

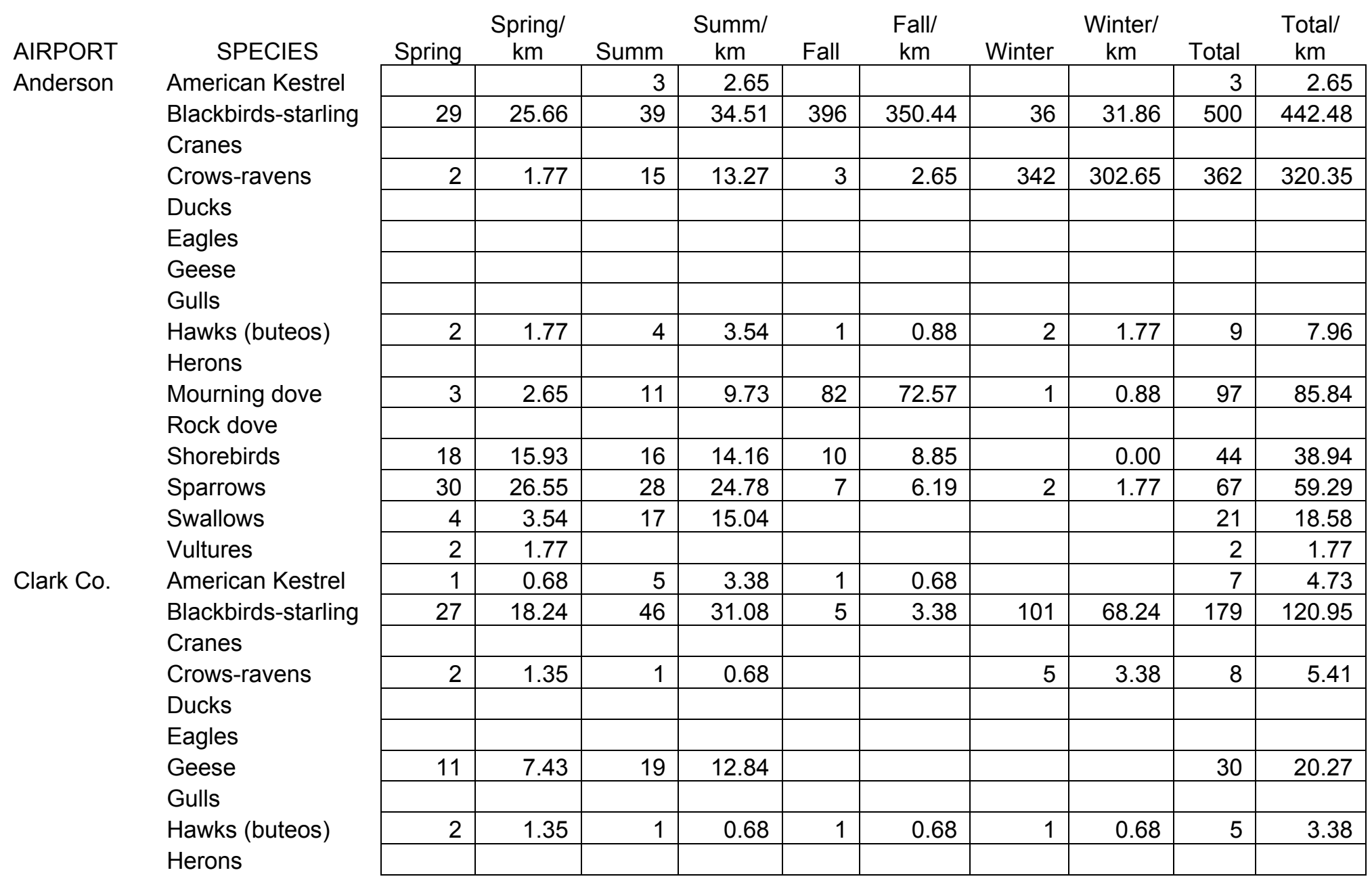


Mourning dove

Rock dove

Shorebirds

Sparrows

Swallows

Vultures

DeKalb Co. American Kestrel

Blackbirds-starling

Cranes

Crows-ravens

Ducks

Eagles

Geese

Gulls

Hawks (buteos)

Herons

Mourning dove

Rock dove

Shorebirds

Sparrows

Swallows

Vultures

Greenwood American Kestrel Blackbirds-starling

Cranes

Crows-ravens

Ducks

Eagles

Geese

Gulls

Hawks (buteos)

Herons

\begin{tabular}{|c|c|c|c|c|c|c|c|c|c|}
\hline 1 & 0.68 & 3 & 2.03 & 27 & 18.24 & 3 & 2.03 & 34 & 22.97 \\
\hline & & 5 & 3.38 & & & 4 & 2.70 & 9 & 6.08 \\
\hline & & 22 & 14.86 & 15 & 10.14 & 5 & 3.38 & 42 & 28.38 \\
\hline 21 & 14.19 & 6 & 4.05 & 12 & 8.11 & 4 & 2.70 & 43 & 29.05 \\
\hline 4 & 2.70 & 17 & 11.49 & & & & & 21 & 14.19 \\
\hline \multirow[t]{2}{*}{2} & 1.35 & & & & & & & 2 & 1.35 \\
\hline & & 2 & 1.31 & & & 1 & 0.65 & 3 & 1.96 \\
\hline 45 & 29.41 & 25 & 16.34 & 2096 & 1369.93 & 50 & 32.68 & 2216 & 1448.37 \\
\hline 7 & 4.58 & 6 & 3.92 & 1 & 0.65 & 16 & 10.46 & 30 & 19.61 \\
\hline 5 & 3.27 & & & & & & & 5 & 3.27 \\
\hline \multirow[t]{3}{*}{4} & 2.61 & & & 20 & 13.07 & & & 24 & 15.69 \\
\hline & & 1 & 0.65 & & & & & 1 & 0.65 \\
\hline & & 1 & 0.65 & 1 & 0.65 & 2 & 1.31 & 4 & 2.61 \\
\hline 2 & 1.31 & 3 & 1.96 & & & & & 5 & 3.27 \\
\hline 1 & 0.65 & 1 & 0.65 & 3 & 1.96 & & & 5 & 3.27 \\
\hline 1 & 0.65 & 72 & 47.06 & 14 & 9.15 & 1 & 0.65 & 88 & 57.52 \\
\hline 37 & 24.18 & 32 & 20.92 & 8 & 5.23 & 2 & 1.31 & 79 & 51.63 \\
\hline 1 & 0.65 & 20 & 13.07 & & & & & 21 & 13.73 \\
\hline \multirow[t]{2}{*}{11} & 7.19 & 18 & 11.76 & 16 & 10.46 & & & 45 & 29.41 \\
\hline & & & & & & 1 & 0.99 & 1 & 0.99 \\
\hline 75 & 74.26 & 132 & 130.69 & 330 & 326.73 & 130 & 128.71 & 667 & 660.40 \\
\hline 1 & 0.99 & 8 & 7.92 & 2 & 1.98 & & & 11 & 10.89 \\
\hline 1 & 0.99 & 2 & 1.98 & & & 6 & 5.94 & 9 & 8.91 \\
\hline \multirow[t]{3}{*}{2} & 1.98 & 1 & 0.99 & & & & & 3 & 2.97 \\
\hline & & & & 1 & 0.99 & 1 & 0.99 & 2 & 1.98 \\
\hline & & 1 & 0.99 & 1 & 0.99 & & & 2 & 1.98 \\
\hline
\end{tabular}


Mourning dove

Rock dove

Shorebirds

Sparrows

Swallows

Vultures

Huntingburg American Kestrel

Blackbirds-starling

Cranes

Crows-ravens

Ducks

Eagles

Geese

Gulls

Hawks (buteos)

Herons

Mourning dove

Rock dove

Shorebirds

Sparrows

Swallows

Vultures

Purdue American Kestrel

Blackbirds-starling

Cranes

Crows-ravens

Ducks

Eagles

Geese

Gulls

Hawks (buteos)

Herons

\begin{tabular}{|r|r|r|r|r|r|r|r|r|r|}
3 & 2.97 & 112 & 110.89 & 31 & 30.69 & 31 & 30.69 & 177 & 175.25 \\
\hline 3 & 2.97 & 7 & 6.93 & 4 & 3.96 & & & 14 & 13.86 \\
\hline 5 & 4.95 & 39 & 38.61 & 3 & 2.97 & & & 47 & 46.53 \\
\hline 12 & 11.88 & 25 & 24.75 & 13 & 12.87 & 6 & 5.94 & 56 & 55.45 \\
\hline 1 & 0.99 & 5 & 4.95 & & & & & 6 & 5.94 \\
\hline 2 & 1.52 & 1 & 0.76 & & & & & 3 & 2.27 \\
\hline 125 & 94.70 & 199 & 150.76 & 269 & 203.79 & 28 & 21.21 & 621 & 470.45 \\
\hline & & & & & & & & & \\
\hline & & & & 1 & 0.76 & 3 & 2.27 & 4 & 3.03 \\
\hline & & & & & & & & & \\
\hline & & & & & & & & & \\
\hline 2 & 1.52 & 13 & 9.85 & & & 40 & 30.30 & 55 & 41.67 \\
\hline & & & & & & & & & \\
\hline & & & & 1 & 0.76 & 2 & 1.52 & 3 & 2.27 \\
\hline 1 & 0.76 & & & & & & & 1 & 0.76 \\
\hline & & 32 & 24.24 & 3 & 2.27 & 6 & 4.55 & 41 & 31.06 \\
\hline & & & & & & & & & \\
\hline 3 & 2.27 & 19 & 14.39 & 6 & 4.55 & 6 & 4.55 & 34 & 25.76 \\
\hline 6 & 4.55 & 20 & 15.15 & 6 & 4.55 & 1 & 0.76 & 33 & 25.00 \\
\hline 12 & 9.09 & 33 & 25.00 & 35 & 26.52 & & & 80 & 60.61 \\
\hline & & & & 1 & 0.76 & & & 1 & 0.76 \\
\hline 1 & 0.54 & 3 & 1.62 & & & 1 & 0.54 & 5 & 2.70 \\
\hline 315 & 170.27 & 102 & 55.14 & 34 & 18.38 & & & 451 & 243.78 \\
\hline & & & & & & 103 & 55.68 & 103 & 55.68 \\
\hline 3 & 1.62 & & & 6 & 3.24 & & & 9 & 4.86 \\
\hline 1 & 0.54 & & & 2 & 1.08 & & & 3 & 1.62 \\
\hline & & & & & & 1 & 0.54 & 1 & 0.54 \\
\hline & & & & & & & & & \\
\hline & & & & & & & & & \\
\hline 1 & 0.54 & 1 & 0.54 & & & & & 2 & 1.08 \\
\hline & & & & & & & & & \\
\hline
\end{tabular}


Mourning dove

Rock dove

Shorebirds

Sparrows

Swallows

Vultures

Putnam Co. American Kestrel

Blackbirds-starling

Cranes

Crows-ravens

Ducks

Eagles

Geese

Gulls

Hawks (buteos)

Herons

Mourning dove

Rock dove

Shorebirds

Sparrows

Swallows

Vultures

Richmond American Kestrel

\begin{tabular}{|r|r|r|r|r|r|r|r|r|r|}
30 & 16.22 & 68 & 36.76 & & & & & 98 & 52.97 \\
\hline & & 6 & 3.24 & & & & & 6 & 3.24 \\
\hline 8 & 4.32 & 15 & 8.11 & 1 & 0.54 & 1 & 0.54 & 25 & 13.51 \\
\hline 27 & 14.59 & 8 & 4.32 & 1 & 0.54 & 2 & 1.08 & 38 & 20.54 \\
\hline 8 & 4.32 & 11 & 5.95 & & & & & 19 & 10.27 \\
\hline & & & & & & 3 & 1.62 & 3 & 1.62 \\
\hline & & 1 & 1.27 & & & & & 1 & 1.27 \\
\hline 27 & 34.18 & 16 & 20.25 & 111 & 140.51 & 30 & 37.97 & 184 & 232.91 \\
\hline & & & & & & & & &
\end{tabular}

Blackbirds-starling

Cranes

Crows-ravens

Ducks

Eagles

Geese

Gulls

Hawks (buteos)

Herons

\begin{tabular}{|c|c|c|c|c|c|c|c|c|c|}
\hline 21 & 34.18 & 16 & 20.25 & 111 & 140.51 & 30 & 31.91 & 184 & 232.91 \\
\hline 4 & 5.06 & 6 & 7.59 & & & 2 & 2.53 & 12 & 15.19 \\
\hline 5 & 6.33 & & & 3 & 3.80 & & & 8 & 10.13 \\
\hline 6 & 7.59 & 15 & 18.99 & & & & & 21 & 26.58 \\
\hline & & & & & & & & & \\
\hline 3 & 3.80 & & & & & & & 3 & 3.80 \\
\hline 6 & 7.59 & 2 & 2.53 & & & & & 8 & 10.13 \\
\hline & & 15 & 18.99 & & & & & 15 & 18.99 \\
\hline 4 & 5.06 & 1 & 1.27 & 1 & 1.27 & 3 & 3.80 & 9 & 11.39 \\
\hline 30 & 37.97 & 19 & 24.05 & 6 & 7.59 & 1 & 1.27 & 56 & 70.89 \\
\hline 11 & 13.92 & 18 & 22.78 & & & & & 29 & 36.71 \\
\hline 1 & 1.27 & & & & & 3 & 3.80 & 4 & 5.06 \\
\hline 103 & 94.50 & 391 & 358.72 & 80 & 73.39 & 33 & 30.28 & 607 & 556.88 \\
\hline 6 & 5.50 & & & & & 2 & 1.83 & 8 & 7.34 \\
\hline & & & & & & & & & \\
\hline & & & & & & & & & \\
\hline & & & & & & & & & \\
\hline & & & & & & & & & \\
\hline & & & & & & & & & \\
\hline & & & & & & & & & \\
\hline
\end{tabular}




\begin{tabular}{|c|c|c|c|c|c|c|c|c|c|c|c|}
\hline & Mourning dove & & & 1 & 0.92 & 95 & 87.16 & 1 & 0.92 & 97 & 88.99 \\
\hline & Rock dove & & & & & & & & & & \\
\hline & Shorebirds & 29 & 26.61 & 37 & 33.94 & 4 & 3.67 & 1 & 0.92 & 71 & 65.14 \\
\hline & Sparrows & 12 & 11.01 & 23 & 21.10 & 11 & 10.09 & 1 & 0.92 & 47 & 43.12 \\
\hline & Swallows & & & 5 & 4.59 & 67 & 61.47 & & & 72 & 66.06 \\
\hline & Vultures & & & & & 2 & 1.83 & & & 2 & 1.83 \\
\hline South Bend & American Kestrel & & & 9 & 11.39 & & & 1 & 1.27 & 10 & 12.66 \\
\hline & Blackbirds-starling & 15 & 18.99 & 79 & 100.00 & 202 & 255.70 & & & 296 & 374.68 \\
\hline & Cranes & & & & & & & & & & \\
\hline & Crows-ravens & 4 & 5.06 & & & 5 & 6.33 & 7 & 8.86 & 16 & 20.25 \\
\hline & Ducks & 2 & 2.53 & & & & & & & 2 & 2.53 \\
\hline & Eagles & & & & & & & & & & \\
\hline & Geese & 2 & 2.53 & & & 11 & 13.92 & & & 13 & 16.46 \\
\hline & Gulls & & & & & & & & & & \\
\hline & Hawks (buteos) & 1 & 1.27 & 2 & 2.53 & 1 & 1.27 & & & 4 & 5.06 \\
\hline & Herons & & & & & & & & & & \\
\hline & Mourning dove & 2 & 2.53 & 1 & 1.27 & 24 & 30.38 & 69 & 87.34 & 96 & 121.52 \\
\hline & Rock dove & & & & & & & & & & \\
\hline & Shorebirds & 4 & 5.06 & 3 & 3.80 & 1 & 1.27 & & & 8 & 10.13 \\
\hline & Sparrows & 11 & 13.92 & 27 & 34.18 & 8 & 10.13 & & & 46 & 58.23 \\
\hline & Swallows & & & 3 & 3.80 & & & & & 3 & 3.80 \\
\hline & Vultures & & & & & & & & & & \\
\hline Warsaw & American Kestrel & 2 & 1.75 & 1 & 0.88 & & & 1 & 0.88 & 4 & 3.51 \\
\hline & Blackbirds-starling & 104 & 91.23 & 103 & 90.35 & 22 & 19.30 & 5 & 4.39 & 234 & 205.26 \\
\hline & Cranes & & & & & & & & & & \\
\hline & Crows-ravens & 1 & 0.88 & 4 & 3.51 & 2 & 1.75 & & & 7 & 6.14 \\
\hline & Ducks & 2 & 1.75 & & & & & & & 2 & 1.75 \\
\hline & Eagles & & & & & & & & & & \\
\hline & Geese & 3 & 2.63 & & & 11 & 9.65 & 22 & 19.30 & 36 & 31.58 \\
\hline & Gulls & 2 & 1.75 & & & & & & & 2 & 1.75 \\
\hline & Hawks (buteos) & 2 & 1.75 & 1 & 0.88 & 2 & 1.75 & 2 & 1.75 & 7 & 6.14 \\
\hline & Herons & 3 & 2.63 & 2 & 1.75 & & & & & 5 & 4.39 \\
\hline
\end{tabular}


Mourning dove

Rock dove

Shorebirds

Sparrows

Swallows

Vultures

\begin{tabular}{|r|r|r|r|r|r|r|r|r|r|}
1 & 0.88 & 37 & 32.46 & 1 & 0.88 & & & 39 & 34.21 \\
\hline 2 & 1.75 & 9 & 7.89 & & & & & 11 & 9.65 \\
\hline 4 & 3.51 & 33 & 28.95 & 7 & 6.14 & & & 44 & 38.60 \\
\hline 4 & 3.51 & 9 & 7.89 & 3 & 2.63 & & & 16 & 14.04 \\
\hline 2 & 1.75 & 7 & 6.14 & 7 & 6.14 & & & 16 & 14.04 \\
\hline & & & & 1 & 0.88 & & & 1 & 0.88 \\
\hline
\end{tabular}


Table 4. Wildlife observed during remote camera surveys at 10 airports in Indiana in 2005-2006. Data are the numbers of observations of new individuals, not abundances. Coyotes and white-tailed deer highlighted. Individuals classified in the Unidentified Mammal or Unidentified Animal categories were only pictures of eyes - no size was determinable.

\begin{tabular}{|c|c|c|c|c|c|c|}
\hline AIRPORT & SPECIES & SPRING & SUMMER & FALL & WINTER & TOTAL \\
\hline \multirow{12}{*}{ Anderson } & \multirow{2}{*}{$\begin{array}{l}\text { American Crow } \\
\text { Common Raccoon }\end{array}$} & & & & 6 & 6 \\
\hline & & 9 & 1 & 3 & 13 & 26 \\
\hline & Coyote & & & & 15 & 15 \\
\hline & \multirow{8}{*}{$\begin{array}{l}\text { Eastern Cottontail } \\
\text { Feral/Domestic Cat } \\
\text { Fox Squirrel } \\
\text { Gray Catbird } \\
\text { Red Squirrel } \\
\text { Unidentified Mammal sp. } \\
\text { Unidentified Squirrel sp. } \\
\text { Virginia Opossum }\end{array}$} & & & & 1 & 1 \\
\hline & & & & & 1 & 1 \\
\hline & & & & 2 & 15 & 17 \\
\hline & & 1 & & & & 1 \\
\hline & & & & 1 & & 1 \\
\hline & & 2 & & 1 & 15 & 17 \\
\hline & & & & & 1 & 1 \\
\hline & & & & 1 & & 1 \\
\hline & White-tailed Deer & 4 & 8 & & 27 & 39 \\
\hline \multirow[t]{7}{*}{ Clark Co. } & Common Raccoon & & & 5 & 1 & 6 \\
\hline & Coyote & & & 1 & 7 & 8 \\
\hline & \multirow{4}{*}{$\begin{array}{l}\text { Fox Squirrel } \\
\text { Great Blue Heron } \\
\text { Unidentified Animal sp. } \\
\text { Unidentified Mammal sp. }\end{array}$} & & & 5 & & 5 \\
\hline & & & & & 1 & 1 \\
\hline & & & & 1 & 2 & 3 \\
\hline & & & & 1 & 2 & 3 \\
\hline & White-tailed Deer & 6 & 3 & 4 & 15 & 28 \\
\hline \multirow[t]{7}{*}{ DeKalb Co. } & \multirow{6}{*}{$\begin{array}{l}\text { Common Raccoon } \\
\text { Domestic Dog } \\
\text { Eastern Cottontail } \\
\text { Fox Squirrel } \\
\text { Unidentified Mammal sp. } \\
\text { Virginia Opossum }\end{array}$} & 1 & & 4 & 2 & 7 \\
\hline & & & & & 1 & 1 \\
\hline & & & & & 1 & 1 \\
\hline & & & & & 1 & 1 \\
\hline & & & & & 2 & 2 \\
\hline & & & & & 1 & 1 \\
\hline & White-tailed Deer & 10 & 2 & 3 & 22 & 37 \\
\hline Greenwood & Common Raccoon & & 17 & 42 & 9 & 68 \\
\hline
\end{tabular}


Huntingburg

Purdue

Putnam Co.

\section{Coyote}

Domestic Dog

Feral/Domestic Cat

Mallard

Unidentified Duck sp.

Unidentified Mammal sp.

Unknown

Woodchuck

Coyote

Eastern Cottontail

Feral/Domestic Cat

Fox Squirrel

Unidentified Bird

Unidentified Mammal sp.

Unknown

White-tailed Deer

Wild Turkey

American Crow

Brown-headed Cowbird

European Starling

Killdeer

Mourning Dove

Red-tailed Hawk

Turkey Vulture

Unidentified Animal

Unidentified Bird

Unidentified Mammal sp.

Unidentified Swallow sp.

Unknown

American Crow

Common Raccoon

Coyote

\begin{tabular}{|c|c|c|c|c|}
\hline & & & 4 & 4 \\
\hline & & 1 & 2 & 3 \\
\hline & & & 1 & 1 \\
\hline & & 2 & & 2 \\
\hline & & 6 & & 6 \\
\hline & & & 2 & 2 \\
\hline 2 & & 1 & & 3 \\
\hline \multirow[t]{5}{*}{1} & & & . & 1 \\
\hline & & & 5 & 5 \\
\hline & & & 3 & 3 \\
\hline & & & 2 & 2 \\
\hline & & & 2 & 2 \\
\hline \multirow[t]{2}{*}{1} & & & & 1 \\
\hline & & & 1 & 1 \\
\hline 2 & & & & 2 \\
\hline \multirow[t]{5}{*}{1} & 2 & & 9 & 12 \\
\hline & & & 1 & 1 \\
\hline & 1 & & & 1 \\
\hline & 1 & & & 1 \\
\hline & 1 & & & 1 \\
\hline \multirow[t]{3}{*}{2} & & & & 2 \\
\hline & 9 & & & 9 \\
\hline & & & 1 & 1 \\
\hline \multirow[t]{2}{*}{2} & 4 & & & 6 \\
\hline & 1 & & & 1 \\
\hline \multirow[t]{2}{*}{3} & & & & 3 \\
\hline & & & 2 & 2 \\
\hline 1 & & & & 1 \\
\hline \multirow[t]{2}{*}{2} & & & & 2 \\
\hline & & 2 & 2 & 4 \\
\hline 3 & & & & 3 \\
\hline 1 & & 1 & 2 & 4 \\
\hline
\end{tabular}


Richmond

South Bend

Warsaw

TOTAL

OBSERVATIONS
Eastern Wood-Pewee

Indigo Bunting

Mourning Dove

Northern Cardinal

Unidentified Animal sp.

Unidentified Bird

Unidentified Mammal sp.

Unidentified Mouse sp.

White-tailed Deer

American Robin

Coyote

Eastern Cottontail

European Starling

Feral/Domestic Cat

Common Raccoon

Eastern Cottontail

Mink

Unidentified Animal

Virginia Opossum

White-tailed Deer

Woodchuck

Common Raccoon

\section{Coyote}

Eastern Cottontail

Feral/Domestic Cat

Unidentified Mammal sp.

\begin{tabular}{|c|c|c|c|c|}
\hline & 1 & & & 1 \\
\hline & 1 & & & 1 \\
\hline & & 1 & & 1 \\
\hline & 1 & & & 1 \\
\hline & & 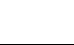 & 2 & 2 \\
\hline & 3 & & & 3 \\
\hline \multirow[t]{2}{*}{1} & & & 1 & 2 \\
\hline & & 1 & & 1 \\
\hline \multirow[t]{2}{*}{4} & & & 19 & 23 \\
\hline & 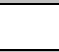 & 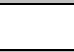 & 1 & 1 \\
\hline \multirow[t]{4}{*}{1} & 1 & & & 2 \\
\hline & & & 2 & 2 \\
\hline & & & 1 & 1 \\
\hline & & & 2 & 2 \\
\hline \multirow[t]{5}{*}{1} & 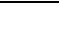 & 2 & 12 & 15 \\
\hline & 3 & & 6 & 9 \\
\hline & & 2 & & 2 \\
\hline & & & 1 & 1 \\
\hline & & & 1 & 1 \\
\hline \multirow[t]{7}{*}{1} & & 2 & & 3 \\
\hline & 3 & 5 & 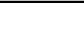 & 8 \\
\hline & & 2 & 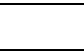 & 2 \\
\hline & & & 2 & 2 \\
\hline & & & 2 & 2 \\
\hline & & & 1 & 1 \\
\hline & & & 1 & 1 \\
\hline 62 & 63 & 101 & 254 & 480 \\
\hline
\end{tabular}


Table 5. Habitat types inside property boundaries at 10 airports in Indiana in 2005-2006. Data represent the percentage of the total airport area. See the text for a description of habitat types.

\begin{tabular}{|c|c|c|c|c|c|c|c|c|c|c|c|}
\hline $\begin{array}{l}\text { HABITAT } \\
\text { TYPE }\end{array}$ & Anderson & $\begin{array}{c}\text { Clark } \\
\text { Co. }\end{array}$ & $\begin{array}{l}\text { DeKalb } \\
\text { Co. }\end{array}$ & Greenwood & Huntingburg & Purdue & $\begin{array}{c}\text { Putnam } \\
\text { Co. }\end{array}$ & Richmond & $\begin{array}{l}\text { South } \\
\text { Bend }\end{array}$ & Warsaw & Average \\
\hline Alfalfa (ALF) & & & & & & & & 10.5 & & & 1.1 \\
\hline $\begin{array}{l}\text { Bare Earth/ } \\
\text { Construction } \\
\text { (BEC) }\end{array}$ & & 0.2 & & 3.3 & 6.7 & 0.4 & & 0.2 & 0.1 & & 1.1 \\
\hline $\begin{array}{l}\text { Cattail Marsh } \\
\text { (CM) }\end{array}$ & & & 0.6 & & & & & & & & 0.1 \\
\hline $\begin{array}{l}\text { Corn Field } \\
\text { (CF) }\end{array}$ & & & 16.0 & 17.7 & 25.4 & & 4.5 & 31.8 & & & 9.5 \\
\hline $\begin{array}{l}\text { Developed } \\
\text { (DEV) }\end{array}$ & 6.0 & 9.3 & 3.9 & 12.1 & 5.5 & 8.7 & 5.1 & 1.4 & 7.7 & 6.1 & 6.6 \\
\hline $\begin{array}{l}\text { Dirt/Gravel } \\
\text { Pile (DGP) }\end{array}$ & & & 0.0 & 0.0 & & 0.1 & 0.1 & 0.0 & & & 0.0 \\
\hline $\begin{array}{l}\text { Ephemeral } \\
\text { Pool (EP) }\end{array}$ & & 0.1 & 0.0 & & & 0.1 & 0.4 & & 0.1 & & 0.1 \\
\hline $\begin{array}{l}\text { Fencerow } \\
\text { (FR) }\end{array}$ & 0.3 & & 0.3 & & & & & & & & 0.1 \\
\hline $\begin{array}{l}\text { Grassy } \\
\text { Swamp (GS) }\end{array}$ & & & 0.6 & & & & & & & & 0.1 \\
\hline $\begin{array}{l}\text { Gravel Road } \\
\text { (GR) }\end{array}$ & & 0.3 & & & 0.1 & 4.0 & & 0.2 & 1.1 & & 0.6 \\
\hline $\begin{array}{l}\text { Hayfield } \\
\text { (HAY) }\end{array}$ & 1.6 & & 7.0 & 17.6 & 5.8 & & & & & & 3.2 \\
\hline $\begin{array}{l}\text { Medium } \\
\text { Grass (MG) }\end{array}$ & 2.7 & 16.0 & & & 5.7 & 0.3 & 12.9 & & 0.4 & 10.1 & 4.8 \\
\hline $\begin{array}{l}\text { Ornamental/ } \\
\text { Shade Tree } \\
\text { (OST) }\end{array}$ & & & 0.5 & 0.0 & & & & & 0.1 & & 0.1 \\
\hline $\begin{array}{l}\text { Permanent } \\
\text { Water (PW) }\end{array}$ & 1.2 & 2.3 & & & 0.3 & 0.0 & & & 0.2 & & 0.4 \\
\hline $\begin{array}{l}\text { Runway } \\
\text { System (RS) }\end{array}$ & 8.1 & 7.2 & 2.8 & 9.0 & 3.8 & 12.9 & 7.1 & 7.0 & 14.5 & 8.4 & 8.1 \\
\hline
\end{tabular}




\begin{tabular}{|c|c|c|c|c|c|c|c|c|c|c|c|}
\hline $\begin{array}{l}\text { Savanna } \\
\text { (SAV) }\end{array}$ & & & & & & & & & 0.9 & & 0.1 \\
\hline $\begin{array}{l}\text { Scrub-Shrub } \\
\text { (SS) }\end{array}$ & 0.4 & 2.0 & 1.4 & 0.5 & & & 3.3 & 0.2 & 0.4 & 0.2 & 0.8 \\
\hline $\begin{array}{l}\text { Short Grass } \\
\text { (SG) }\end{array}$ & 34.8 & 50.6 & 20.8 & 25.5 & 24.0 & 73.4 & 16.8 & 9.3 & 75.5 & 71.6 & 40.2 \\
\hline $\begin{array}{l}\text { Sorghum } \\
\text { Field (SGF) }\end{array}$ & & & & & 10.2 & & & & & & 1.0 \\
\hline $\begin{array}{l}\text { Soybean } \\
\text { Field (SOY) }\end{array}$ & 34.4 & & 12.9 & 8.1 & 3.8 & & 6.5 & 37.7 & & & 10.3 \\
\hline $\begin{array}{l}\text { Stone Swale } \\
\text { (STS) }\end{array}$ & & & & & 0.0 & & & & 0.1 & & 0.0 \\
\hline $\begin{array}{l}\text { Tall Grass } \\
\text { (TG) }\end{array}$ & 6.2 & 0.8 & & 4.1 & 4.0 & 0.0 & 29.7 & & 0.5 & 0.2 & 4.6 \\
\hline $\begin{array}{l}\text { Weedy Ditch } \\
\text { (WD) }\end{array}$ & 0.6 & 0.6 & 0.5 & 1.9 & & 0.1 & 1.2 & 0.0 & 0.0 & & 0.5 \\
\hline $\begin{array}{l}\text { Wheat Field } \\
(\text { WF) }\end{array}$ & & & 1.0 & & & & & & & 3.6 & 0.5 \\
\hline Woodlot (W) & 3.8 & 10.9 & 20.2 & & 4.6 & & 12.3 & & & & 5.2 \\
\hline Other (OTH) & & & 11.6 & & & & & 1.7 & & & 1.3 \\
\hline TOTAL & 100.1 & 100.3 & 100.1 & 99.8 & 99.9 & 100.0 & 99.9 & 100.0 & 101.6 & 100.2 & 100.2 \\
\hline
\end{tabular}


Table 6. Presence of wildlife attractants at 10 airports in Indiana in 2005-2006.

\begin{tabular}{|c|c|c|c|c|c|c|c|c|c|c|c|}
\hline ATTRACTANT & Anderson & $\begin{array}{l}\text { Clark } \\
\text { Co. }\end{array}$ & $\begin{array}{l}\text { DeKalb } \\
\text { Co. }\end{array}$ & Greenwood & Huntingburg & Purdue & $\begin{array}{l}\text { Putnam } \\
\text { Co. }\end{array}$ & Richmond & $\begin{array}{l}\text { South } \\
\text { Bend }\end{array}$ & Warsaw & $\begin{array}{l}\text { Total } \\
\text { Airports }\end{array}$ \\
\hline Crop Field & $\mathrm{X}$ & & $\mathrm{X}$ & $\mathrm{X}$ & $\mathrm{x}$ & & $x$ & $\mathrm{X}$ & & $x$ & 7 \\
\hline $\begin{array}{l}\text { Woodlot } \\
\text { Refugia }\end{array}$ & $X$ & $X$ & $x$ & $x$ & $x$ & & $X$ & & $x$ & & 7 \\
\hline $\begin{array}{l}\text { Permanent } \\
\text { Standing Water }\end{array}$ & & & $X$ & & $x$ & $X$ & & & $X$ & & 4 \\
\hline $\begin{array}{l}\text { Ephemeral } \\
\text { Standing Water }\end{array}$ & & $X$ & $X$ & $X$ & $x$ & $X$ & $x$ & $X$ & $x$ & & 8 \\
\hline $\begin{array}{l}\text { Permanent } \\
\text { Open Stream }\end{array}$ & $X$ & $X$ & & $x$ & & & & & & & 3 \\
\hline $\begin{array}{l}\text { Ephemeral } \\
\text { Open Stream }\end{array}$ & $X$ & $x$ & & & & & $X$ & & & & 3 \\
\hline $\begin{array}{l}\text { Open Refuse } \\
\text { Container }\end{array}$ & & $X$ & & & & & $x$ & & & & 2 \\
\hline Open Building & & & & $x$ & & & & & & & 1 \\
\hline Open Culvert & $X$ & $X$ & $X$ & & $\mathrm{X}$ & $\mathrm{X}$ & $X$ & & $\mathrm{X}$ & $\mathrm{X}$ & 8 \\
\hline Brush Pile & $x$ & & $x$ & & & & & & $x$ & & 3 \\
\hline Gravel Pile & & & $\mathrm{X}$ & & $\mathrm{X}$ & $x$ & $\mathrm{X}$ & $x$ & & & 5 \\
\hline $\begin{array}{l}\text { Total } \\
\text { Attractants }\end{array}$ & 6 & 6 & 7 & 5 & 6 & 4 & 7 & 3 & 5 & 2 & \\
\hline
\end{tabular}


Table 7. Fence characteristics and percentage of perimeter fenced at 10 airports in Indiana in 2005-2006. See the text for description of fence types.

AIRPORT

Anderson

Clark Co.

DeKalb Co.

Greenwood

Huntingburg

Purdue

Putnam Co.

Richmond

South Bend

Warsaw

Total Airports

\begin{tabular}{|c|c|c|c|c|c|c|c|c|c|c|}
\hline Type A & Type B & Type C & Type D & Type E & Type F & Type G & Type H & Type I & Type J & Total \\
\hline & & & & & & & 4.1 & 23.6 & 49.2 & 76.9 \\
\hline & & & & & & 42.9 & & & & 42.9 \\
\hline & & & & & & 22.5 & & & & 22.5 \\
\hline & & & & & & & 98.4 & & 0.8 & 99.2 \\
\hline & & & & & & 1.1 & & 0.6 & 42.0 & 43.7 \\
\hline & & 80.9 & 2.3 & 0.1 & 1.6 & & 1.4 & & 6.7 & 93.0 \\
\hline 24.8 & & & & & & & & & 26.9 & 51.7 \\
\hline & & & & & & 2.5 & & 5.0 & & 7.5 \\
\hline & 77.7 & & & & & 12.6 & & & & 90.3 \\
\hline & & & & & & 2.4 & 95.7 & 1.9 & & 100.0 \\
\hline 1 & 1 & 1 & 1 & 1 & 1 & 6 & 4 & 4 & 5 & \\
\hline
\end{tabular}


Table 8. Type and number of fence openings at 10 airports in Indiana in 2005-2006. Shaded airports were not evaluated because they had $<25 \%$ fenced perimeter with chain-link-type fencing.

\begin{tabular}{|c|c|c|c|c|c|c|c|c|c|}
\hline \multirow{3}{*}{$\begin{array}{l}\text { AIRPORT } \\
\text { Anderson }{ }^{1} \\
\text { Clark Co. }\end{array}$} & Break & Culvert & Dig-hole & Gap & Hole & Warp & Other & Total & Total/100m \\
\hline & 3 & & & 8 & & & & 9 & 0.2 \\
\hline & 3 & 3 & 3 & 5 & & & & 14 & 0.5 \\
\hline \multicolumn{10}{|l|}{ DeKalb Co. } \\
\hline Greenwood & & & & 13 & & 2 & & 15 & 0.3 \\
\hline \multicolumn{10}{|l|}{ Huntingburg } \\
\hline Purdue & & 2 & 16 & 35 & 59 & 14 & & 126 & 1.3 \\
\hline \multicolumn{10}{|l|}{ Putnam Co. } \\
\hline Richmond & & & & & & & & & \\
\hline \multirow{2}{*}{$\begin{array}{l}\text { South Bend } \\
\text { Warsaw }^{2}\end{array}$} & & & 35 & 27 & & 5 & & 67 & 0.5 \\
\hline & 2 & & 7 & 22 & & 8 & 3 & 42 & 0.5 \\
\hline
\end{tabular}

1 - Two unfenced sections of $>500$ meters in length also occurred at this airfield. The 3 breaks indicated include a $\sim 1$ meter break and 2 breaks at the junction of the fence and mounds road

2 - Two breaks of $\sim 1$ meter each were occurred at the junction of the terminal with the fence at this airfield 
Table 9. Habitat characteristics within a 10-km radius of three airports in Indiana in 2005-2006. Data represent percentage of total area. See the text for a description of habitat types.

\section{HABITAT TYPE}

Barren (B)

Forest/Woods (F/W)

Grassland (G)

Heavy Development (HD)

Moderate Development (MD)

Open Wetland (OW)

Pasture $(P)$

Pond/River (P/R)

Recreational Field (RF)

Row Crop (RC)

Shrubland (S)

Wooded Wetland (WW)

Other (O)

\begin{tabular}{|c|c|c|}
\multicolumn{1}{c}{ DeKalb Co. } & \multicolumn{1}{c}{ South Bend } & Warsaw \\
\hline 0.6 & 0.4 & 0.3 \\
\hline 17.2 & 16.4 & 11.3 \\
\hline 12.6 & 3.3 & 5.0 \\
\hline 1.7 & 7.1 & 2.0 \\
\hline 15.2 & 34.9 & 17.0 \\
\hline 1.1 & 1.2 & 2.7 \\
\hline 2.2 & 0.6 & 1.8 \\
\hline 1.5 & 0.9 & 4.2 \\
\hline 1.1 & 2.7 & 1.1 \\
\hline 42.9 & 27.4 & 49.3 \\
\hline 2.9 & 3.6 & 2.3 \\
\hline 1.0 & 0.7 & 2.7 \\
\hline 0 & 0.8 & 0.2 \\
\hline
\end{tabular}


Table 10 (ELECTRONIC ONLY; LOCATED ON CD). Strike records from FAA Strike Database of 10 airports in Indiana in 20052006. Data were compiled at the Sandusky Field Office, USDA/APHIS/WS/National Wildlife Research Center. 
Table 11. Summary of deer and coyote observations at 10 airports in Indiana in 2005-2006. Data represent the number of observations of deer and coyotes within airport boundaries.

\begin{tabular}{|c|c|c|c|c|c|c|c|c|}
\hline \multirow{2}{*}{ AIRPORT } & \multicolumn{4}{|c|}{ COYOTE } & \multicolumn{4}{|c|}{ DEER } \\
\hline & CAMERA & SPOTLIGHT & OPPORT. & TOTAL & CAMERA & SPOTLIGHT & OPPORT. & TOTAL \\
\hline Anderson & 15 & & 1 & 16 & 39 & 50 & 3 & 92 \\
\hline \multirow{3}{*}{$\begin{array}{l}\text { Clark Co. } \\
\text { DeKalb Co. } \\
\text { Greenwood }\end{array}$} & 8 & 9 & 11 & 28 & 28 & 26 & & 54 \\
\hline & & & 1 & 1 & 37 & 18 & 9 & 64 \\
\hline & 4 & & & 4 & & & & 0 \\
\hline \multirow{2}{*}{$\begin{array}{l}\text { Huntingburg } \\
\text { Purdue }\end{array}$} & 5 & 2 & 3 & 10 & 12 & 4 & & 16 \\
\hline & & & & 0 & & & & 0 \\
\hline \multirow{4}{*}{$\begin{array}{l}\text { Putnam Co. } \\
\text { Richmond } \\
\text { South Bend } \\
\text { Warsaw }\end{array}$} & 4 & & 2 & 6 & 23 & 19 & 4 & 46 \\
\hline & 2 & & 1 & 3 & & 1 & & 1 \\
\hline & & 2 & 1 & 3 & 3 & 7 & 14 & 24 \\
\hline & 2 & 1 & 1 & 4 & & & & 0 \\
\hline
\end{tabular}


Plates 1-5. Observations of wildlife at 10 airports in Indiana. Photographs were taken with remote cameras in 2005-2006.

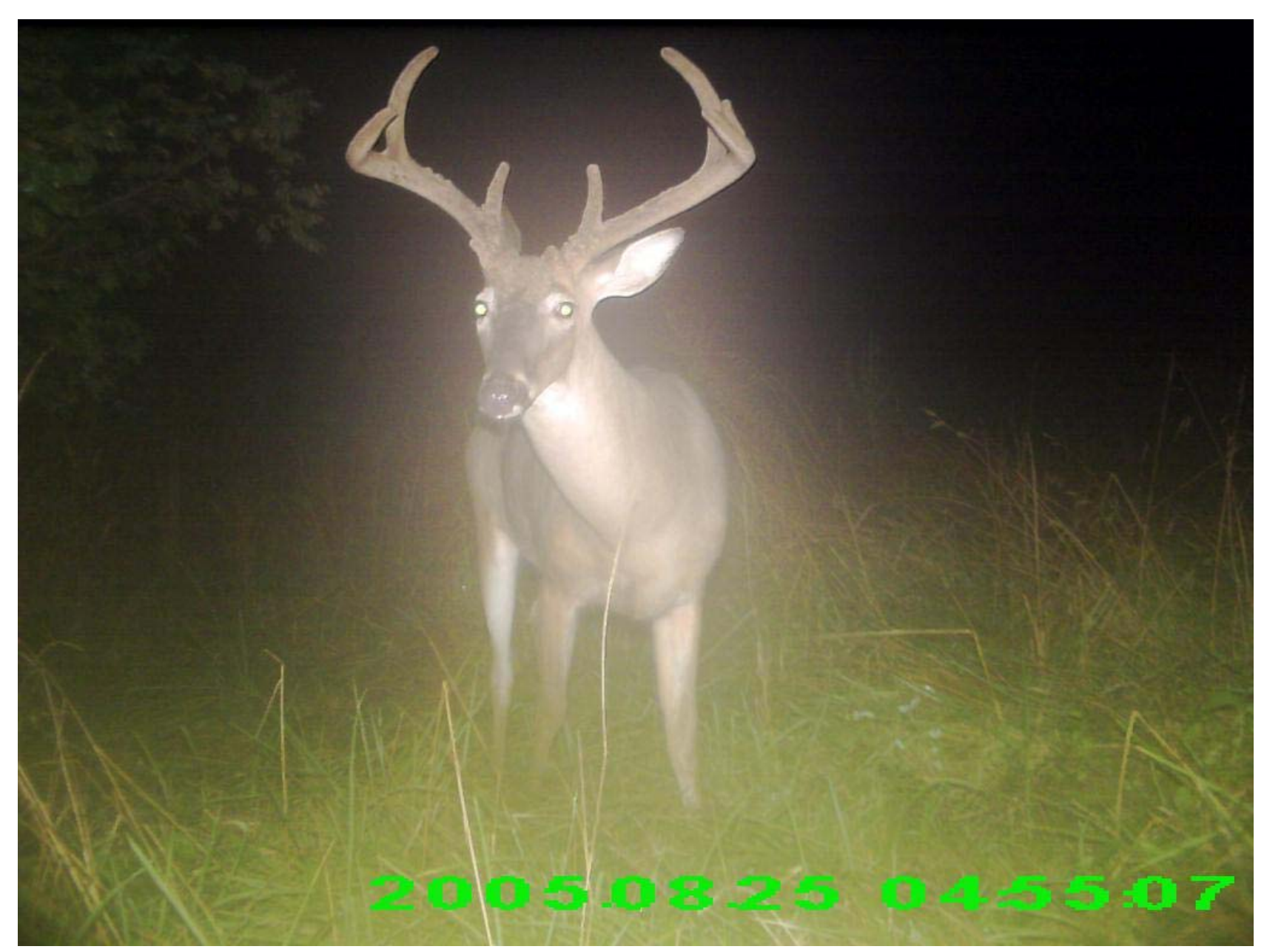

1. White-tailed deer near wooded margin. 


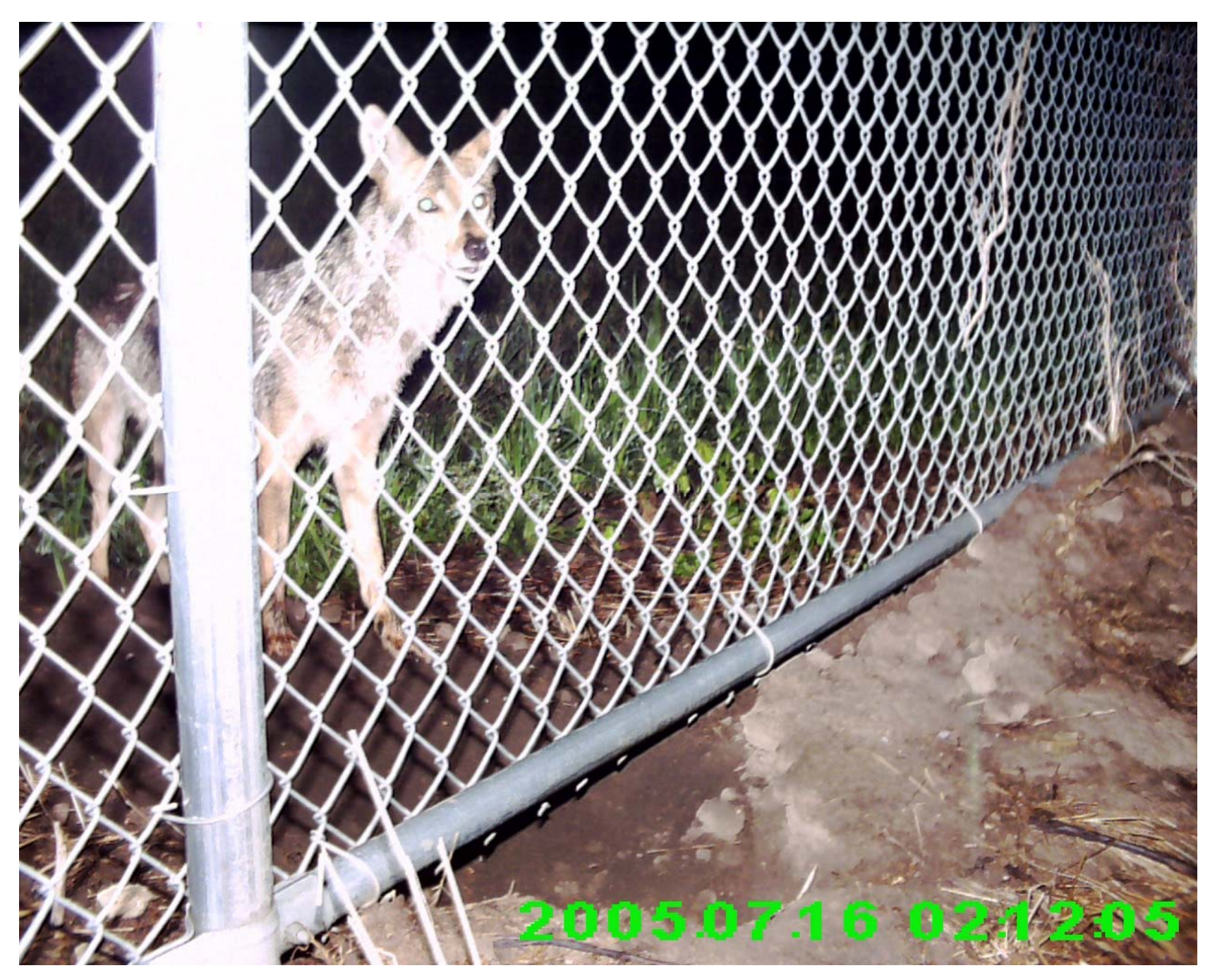

2. Coyote near dig-hole under fence. 


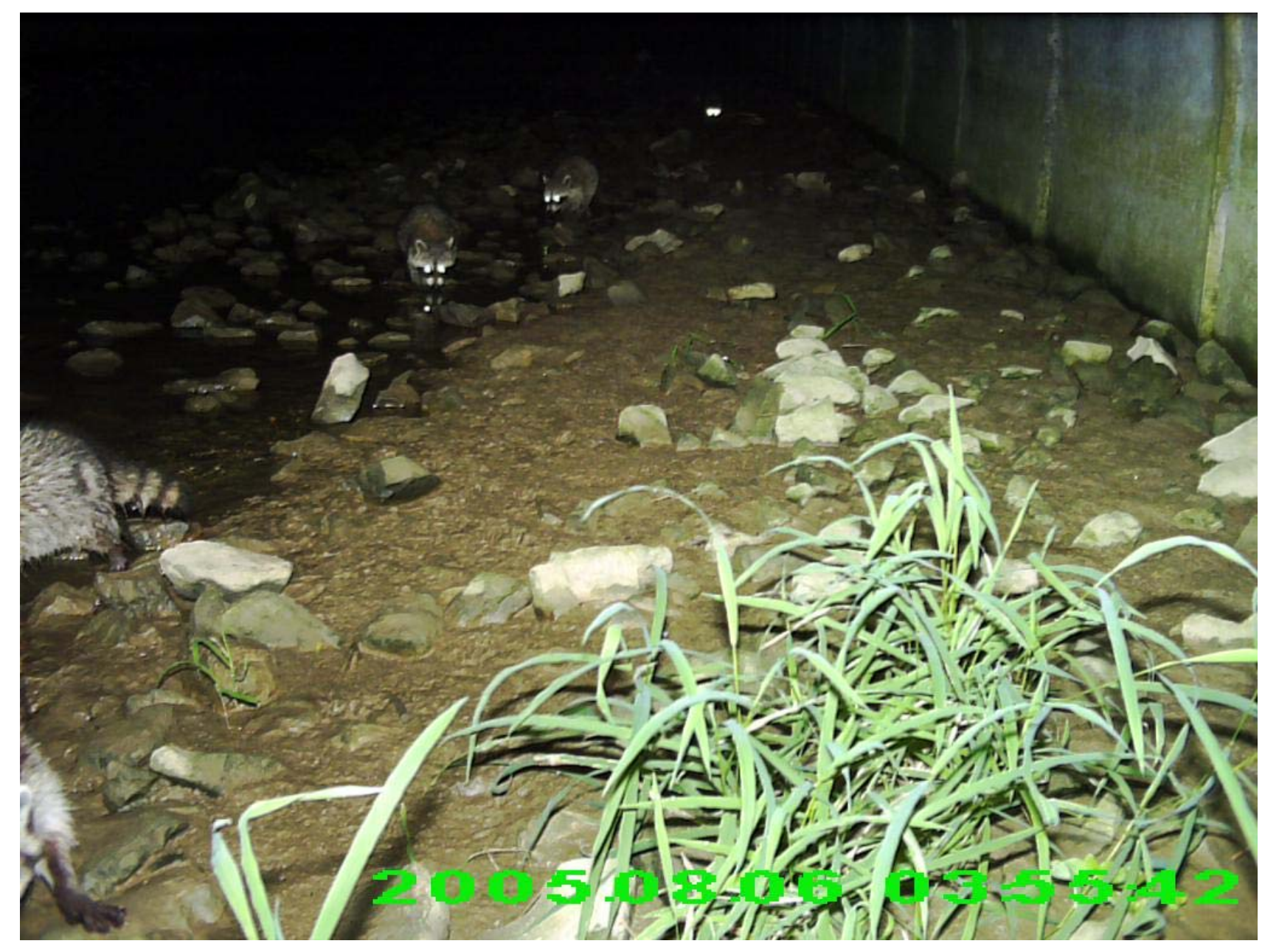

3. Raccoons traveling through airport culvert. 


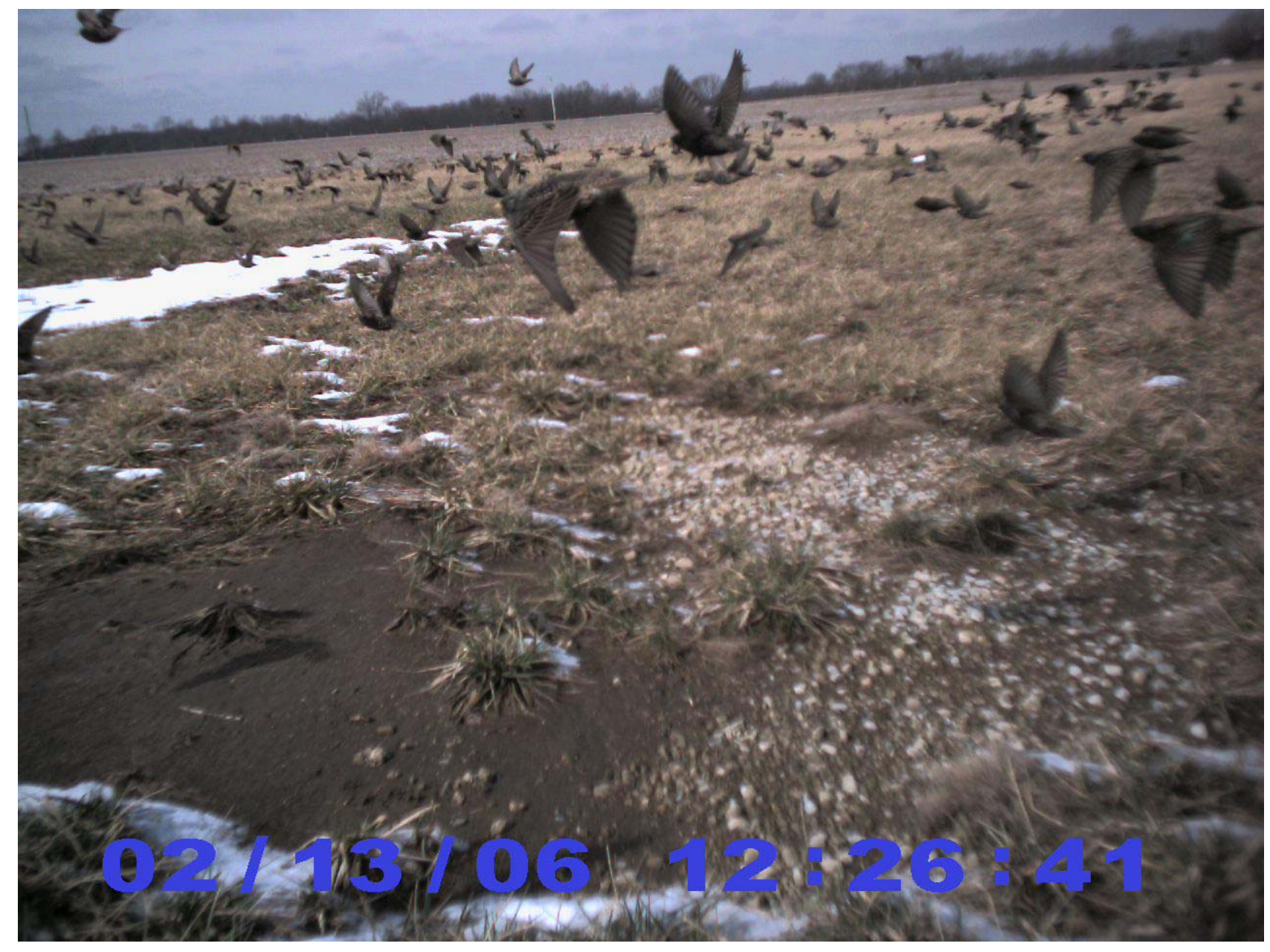

4. Flock of European starlings inside airport property. 


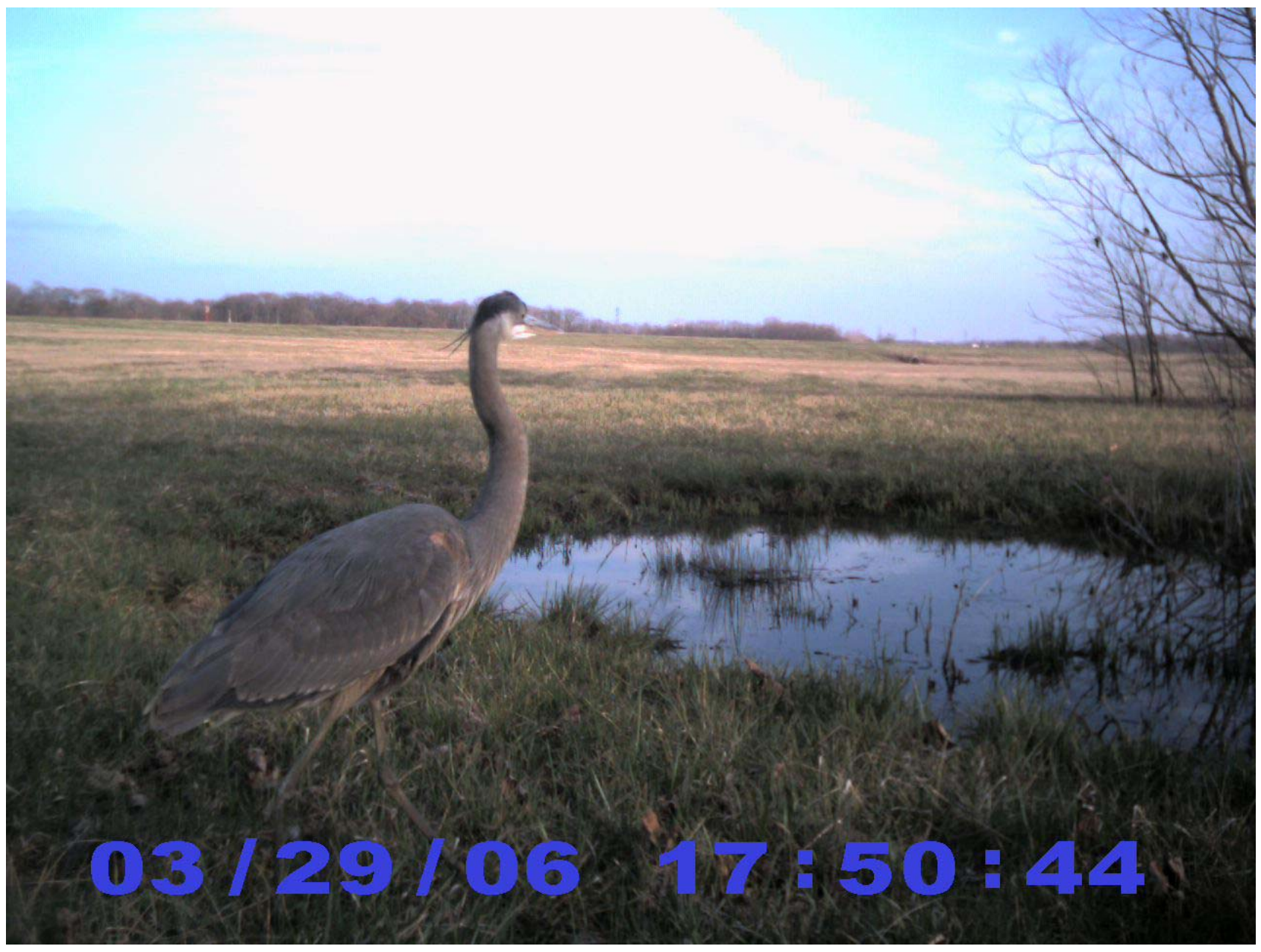

5. Great blue heron near water-filled depression inside airport property. 
Figures 1-10.

Locations of sampling points and routes for remote-camera, bird, and spotlight surveys conducted at 10 airports as part of the evaluation of wildlife hazards at general aviation airports in Indiana, 2005-2006. 


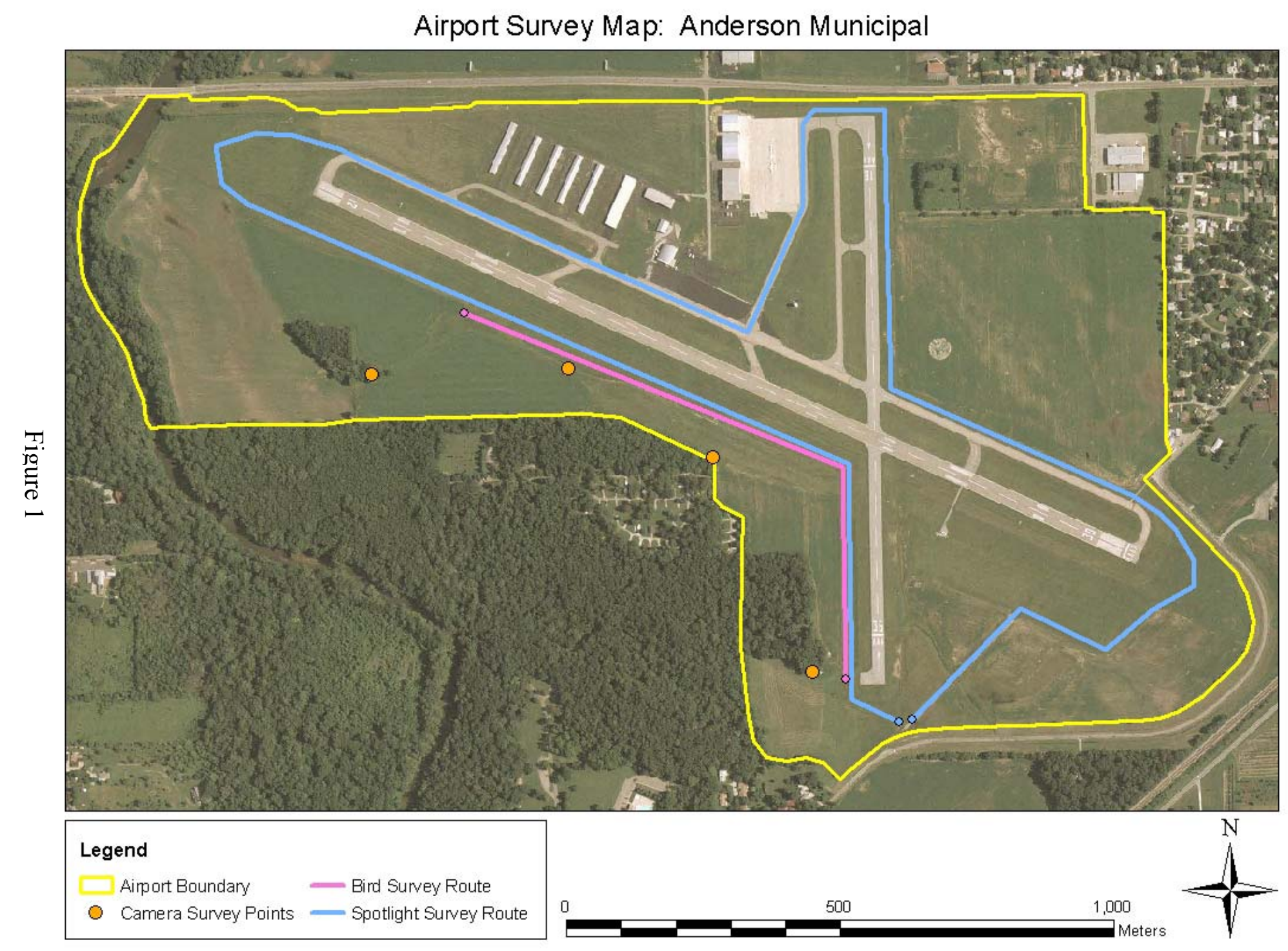




\section{Airport Survey Map: Clark County}

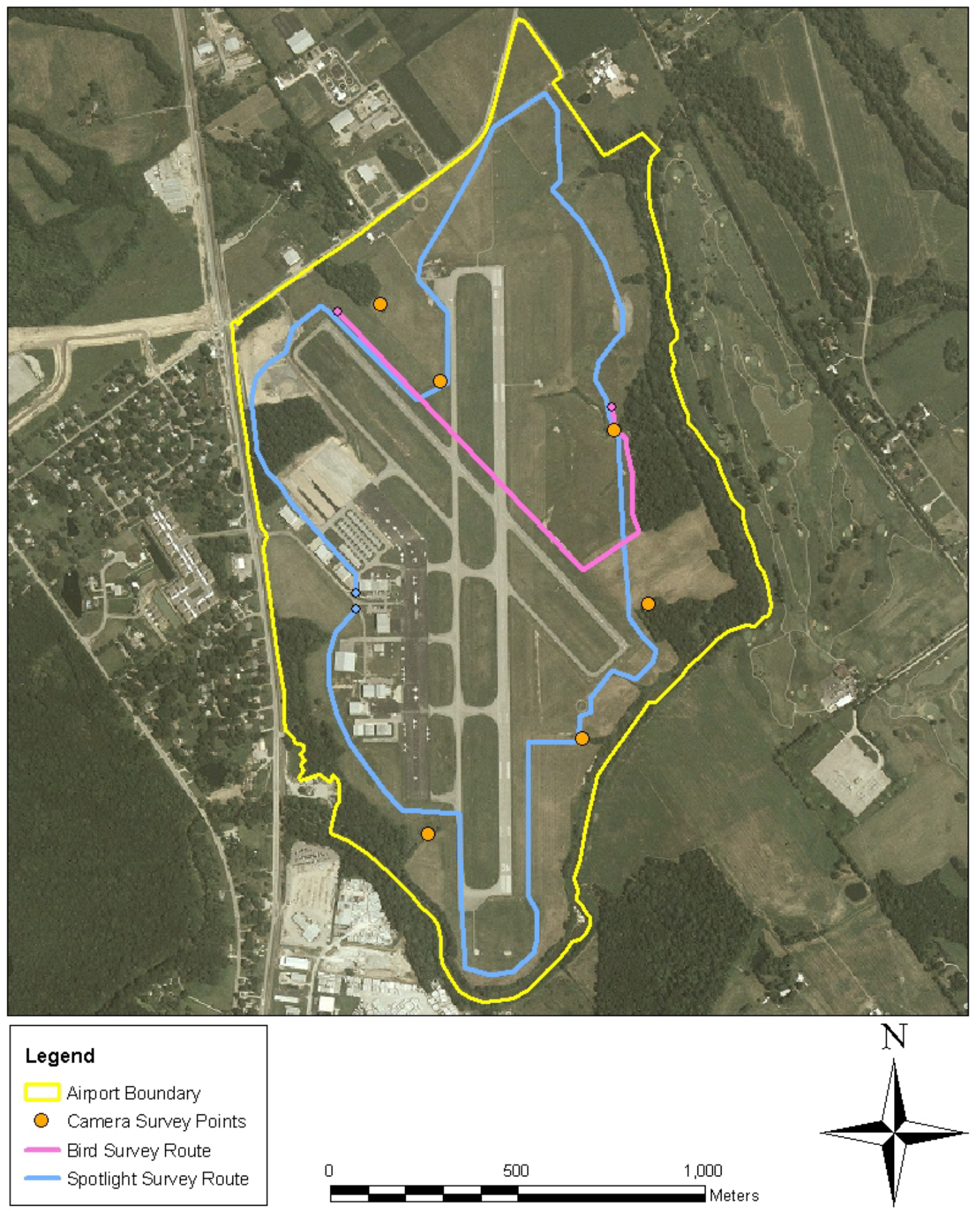

Figure 2 


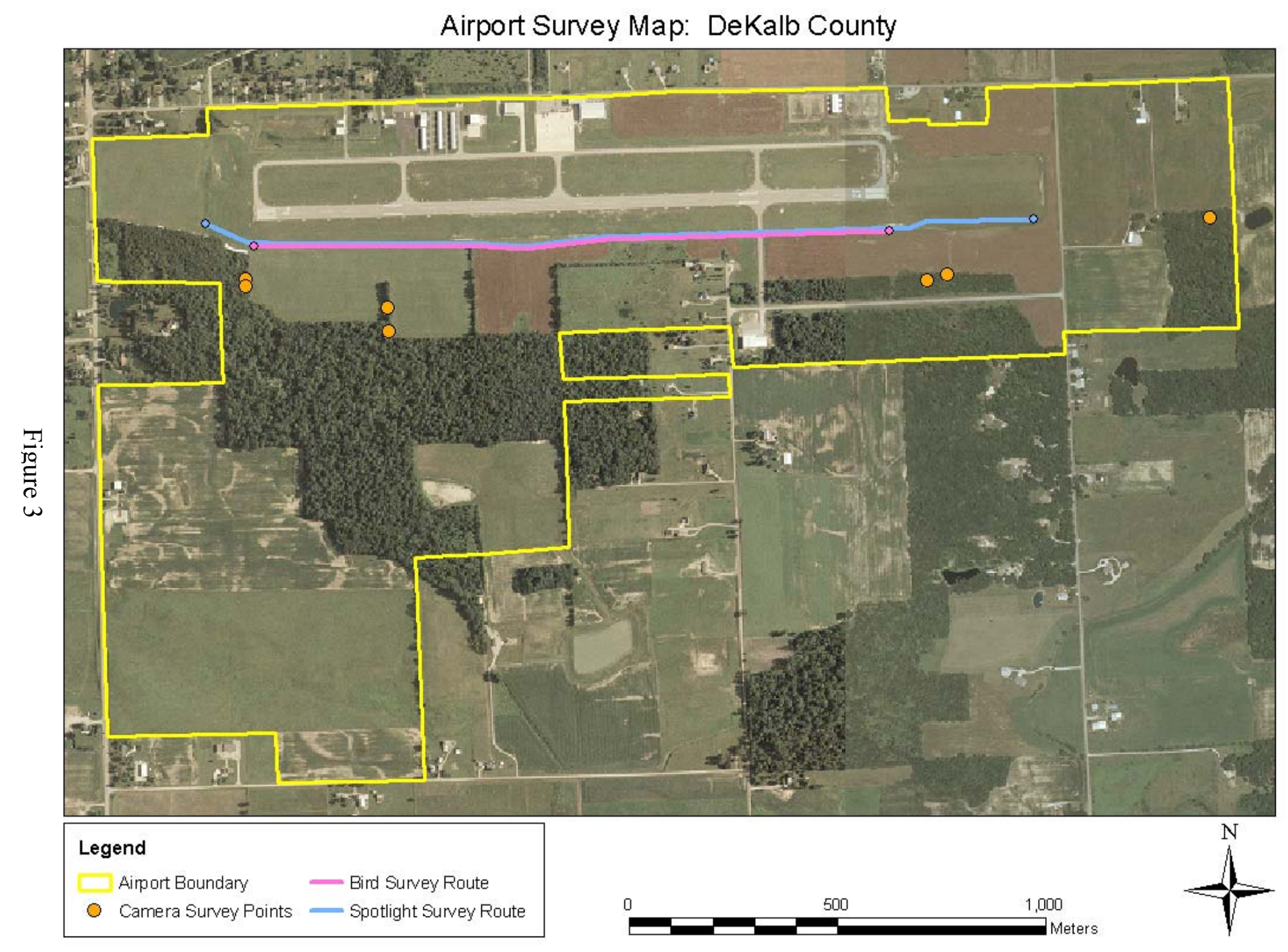




\section{Airport Survey Map: Greenwood Municipal}

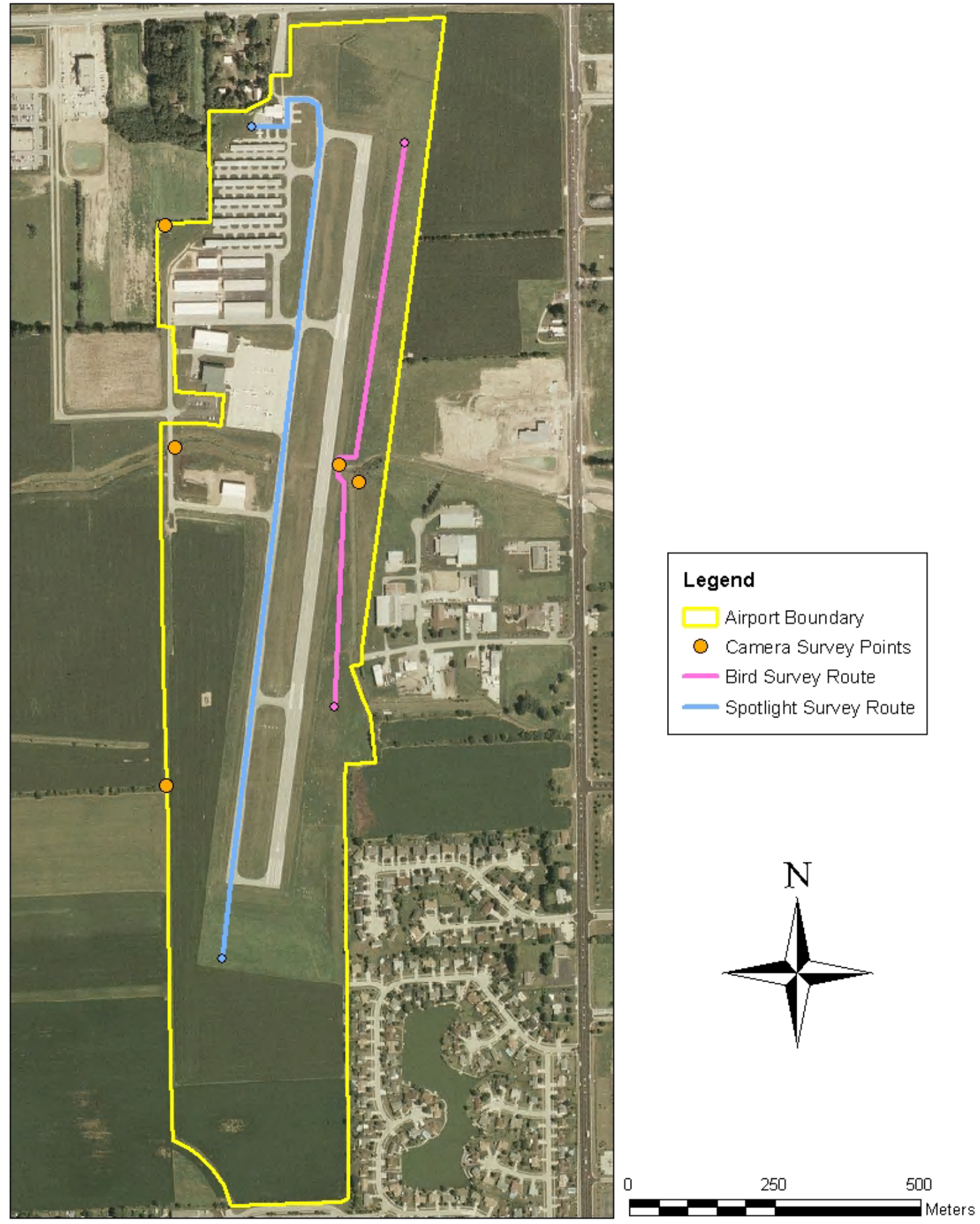

Figure 4 


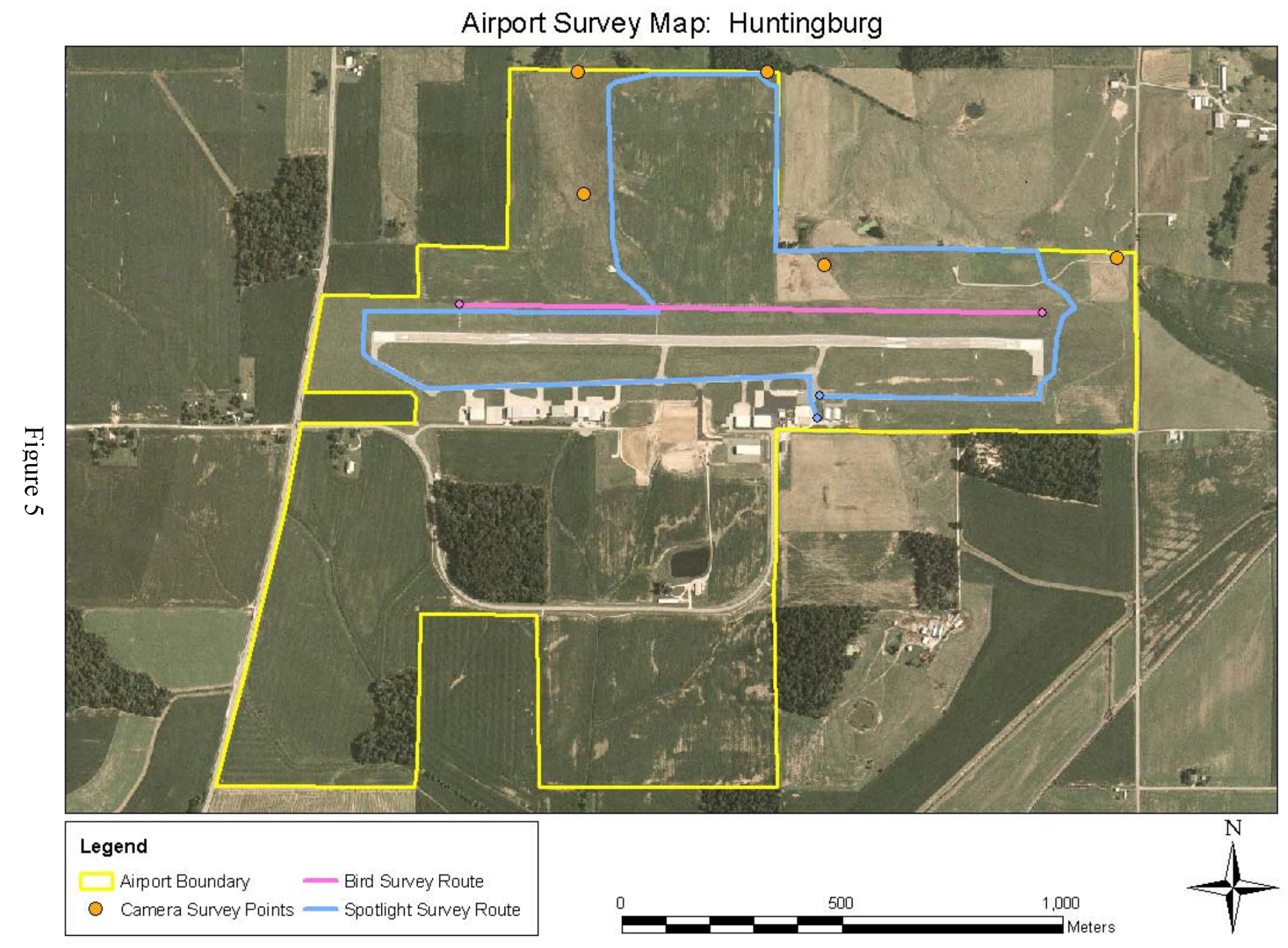


Airport Survey Map: Purdue University

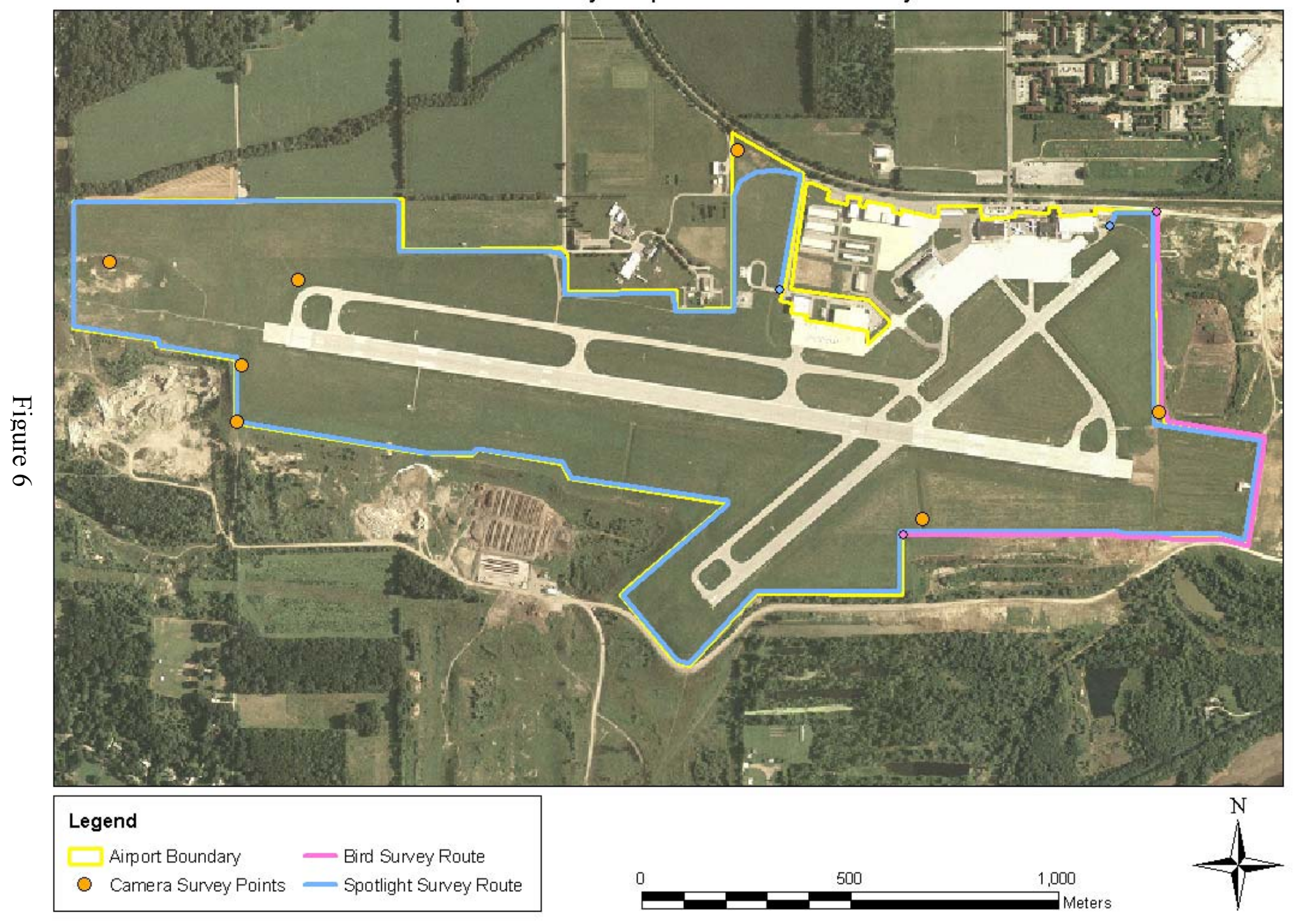




\section{Airport Survey Map: Putnam County}

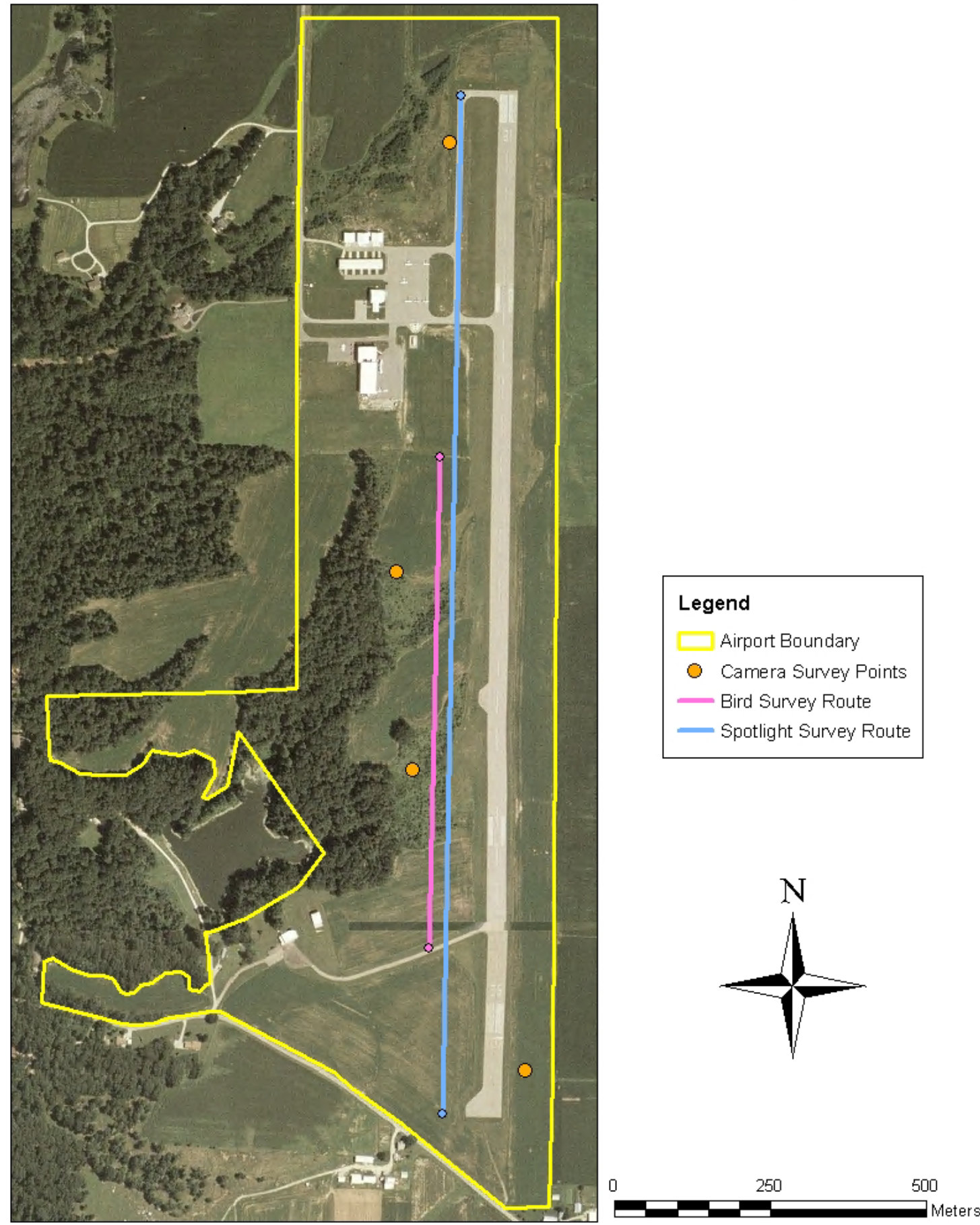

Figure 7 


\section{Airport Survey Map: Richmond Municipal}

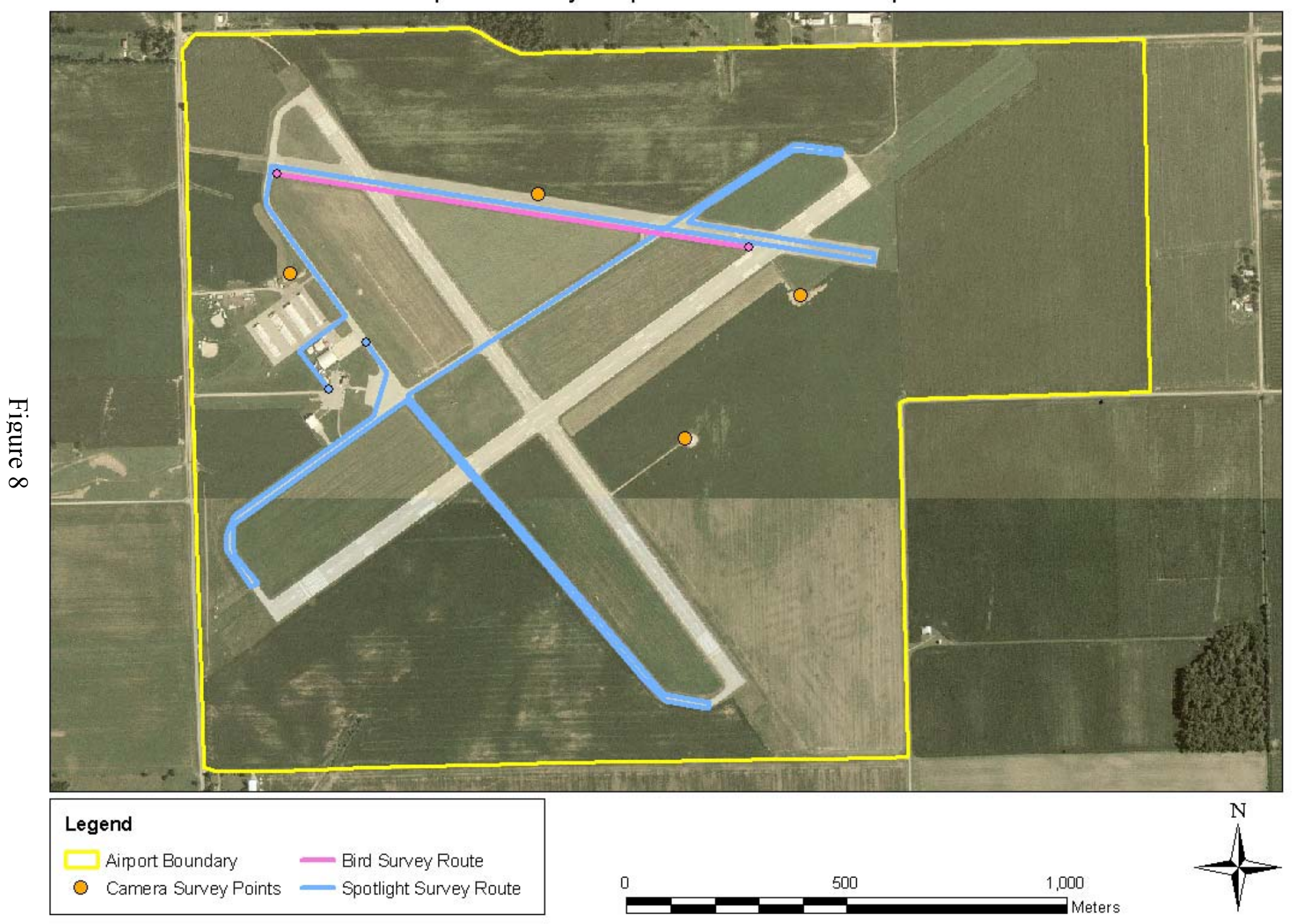


Airport Survey Map: South Bend Regional

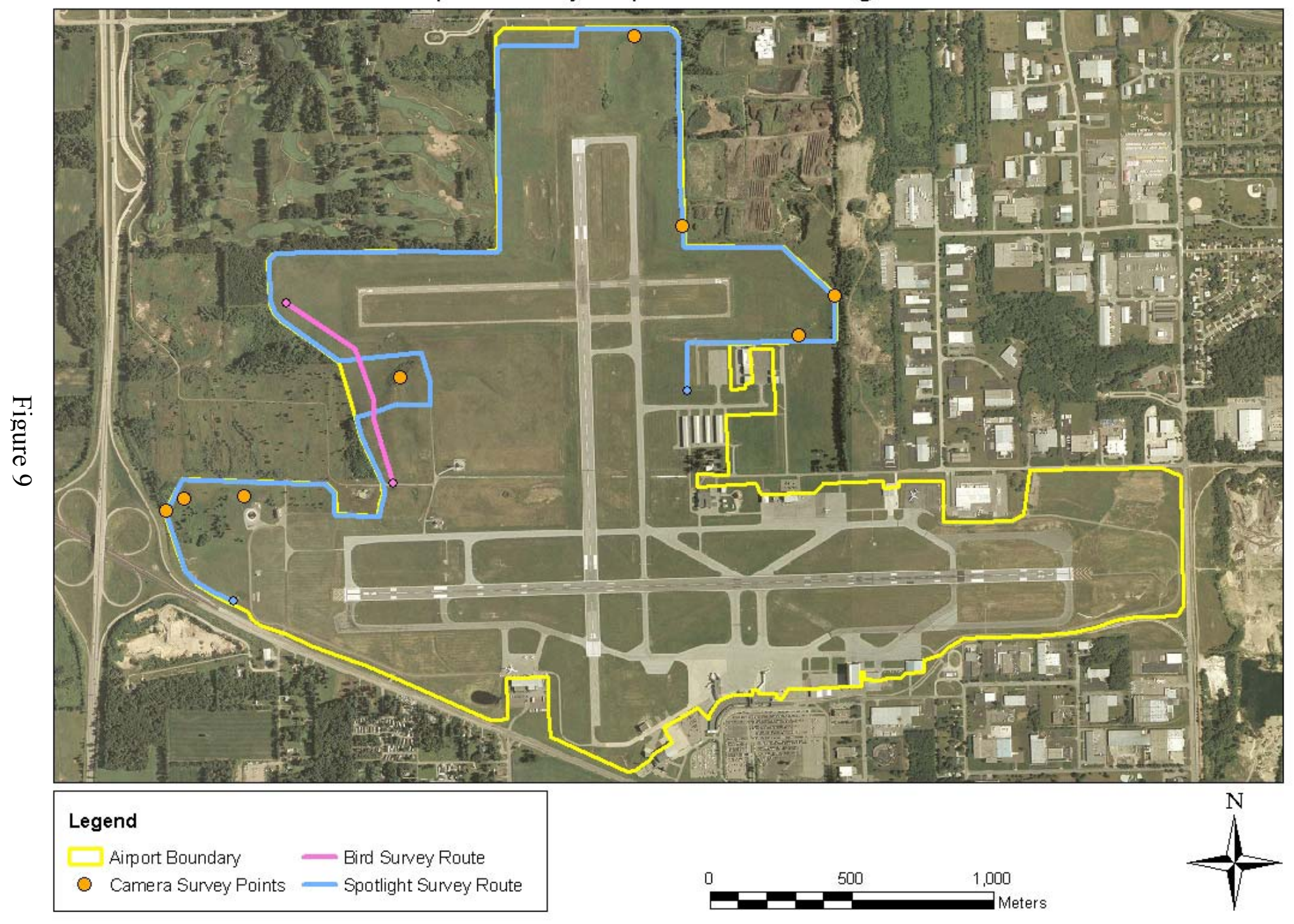


Airport Survey Map: Warsaw Municipal

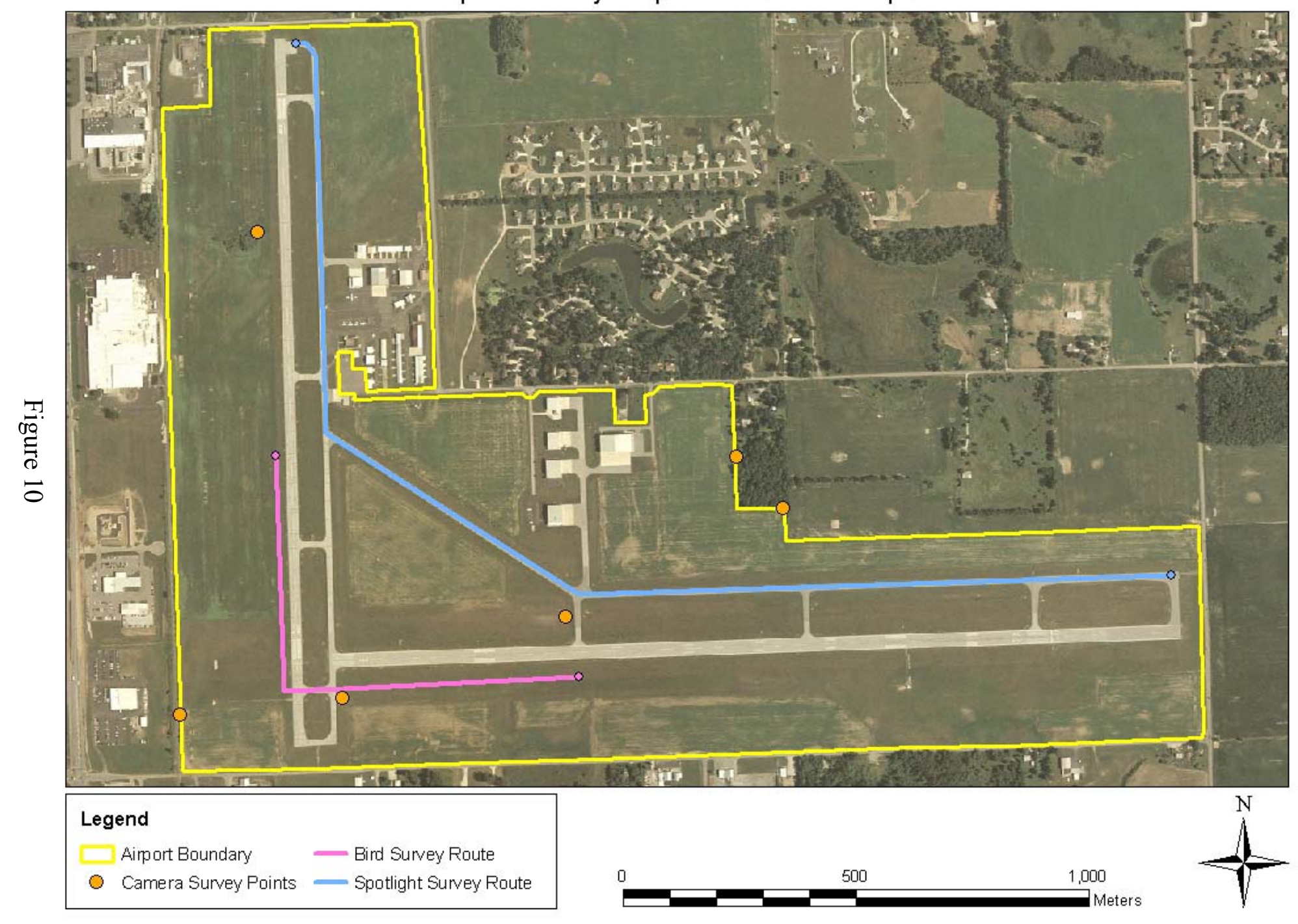


Figures 11-30.

Distribution of habitat patches and relative proportion of habitat types observed within 10 airport properties as part of the evaluation of wildlife hazards at general aviation airports in Indiana, 2005-2006. Color schemes for each figure were developed independently. 
Airport Habitat Map: Anderson Municipal

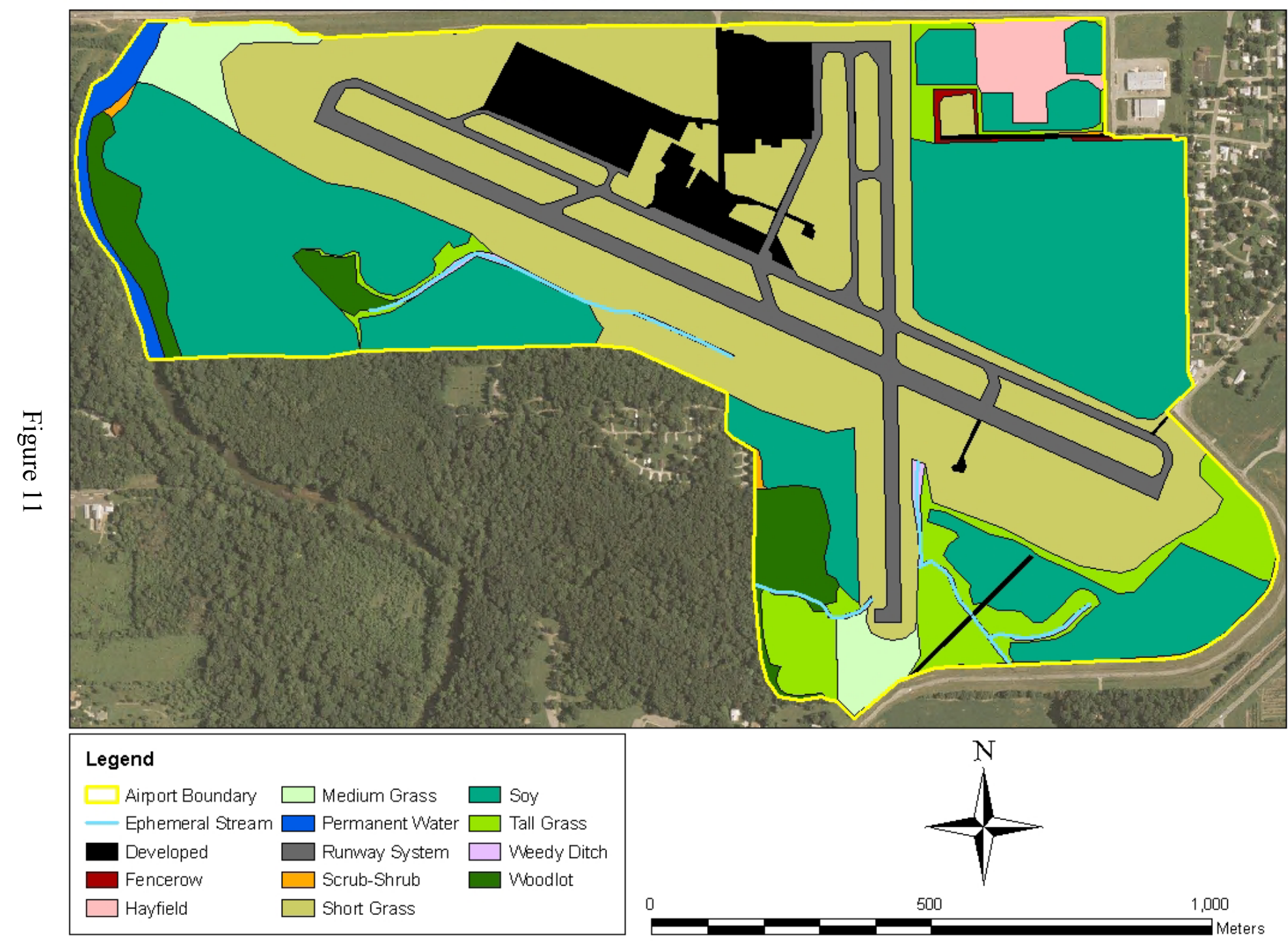




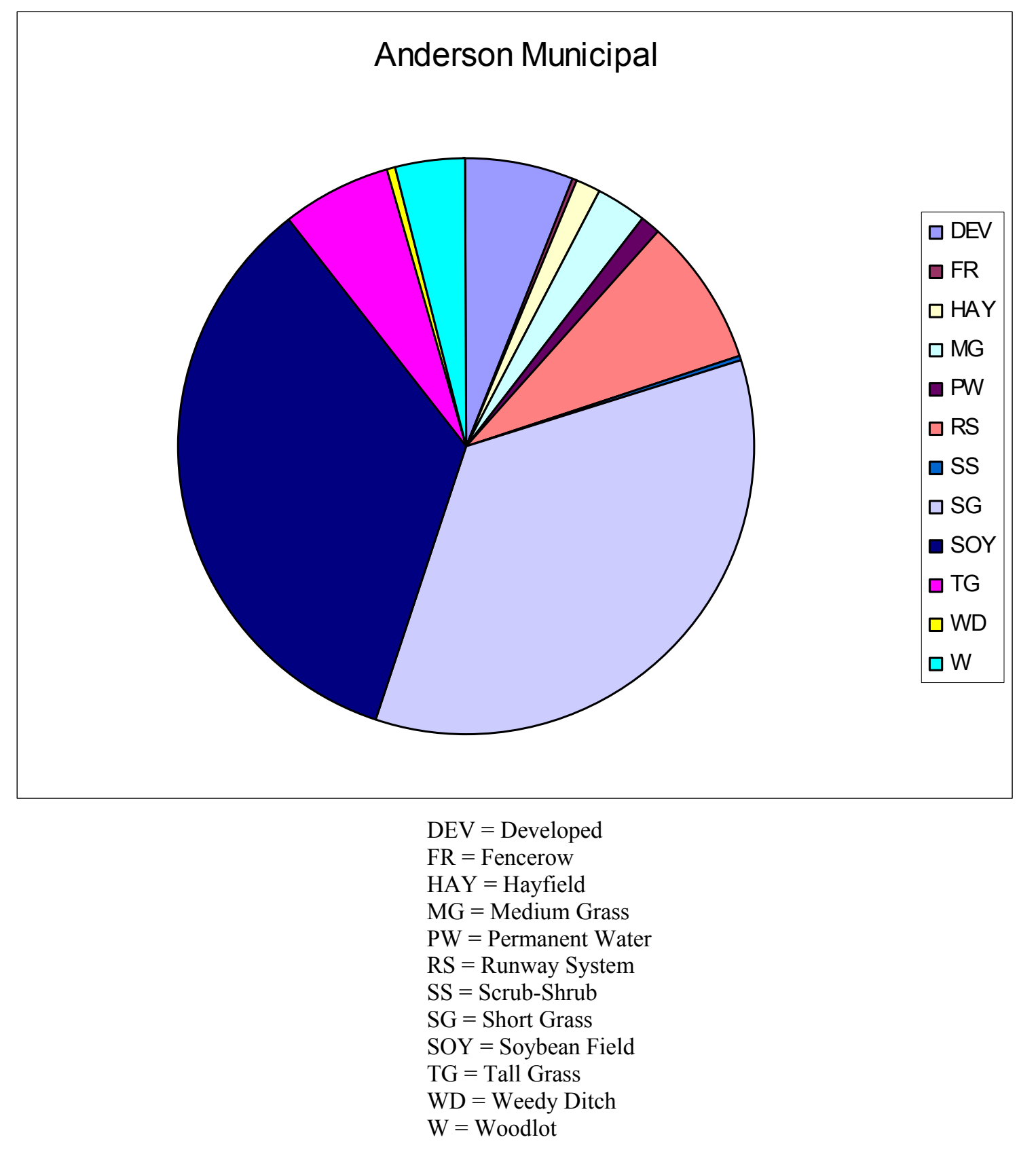

Figure 12 


\section{Airport Habitat Map: Clark County}

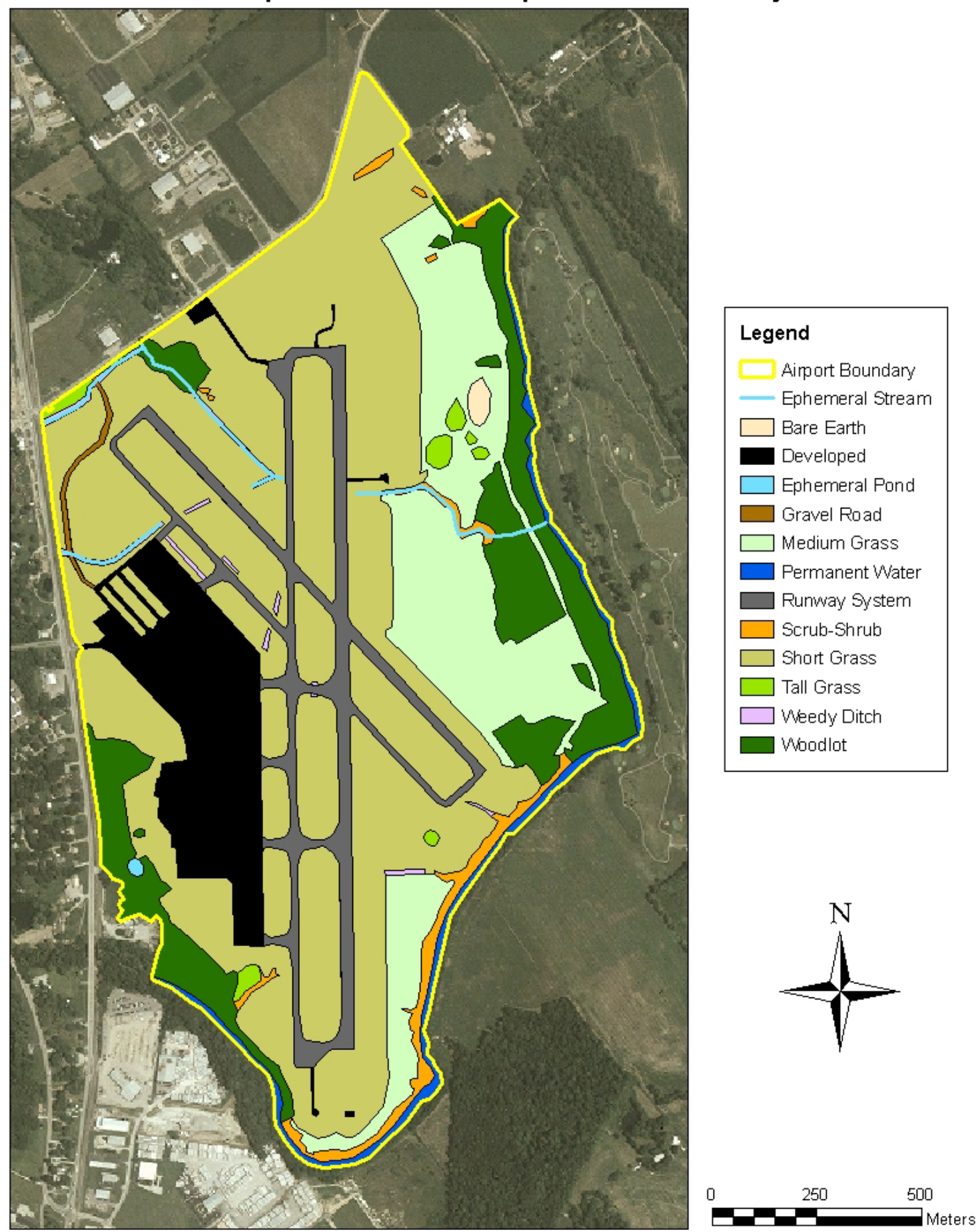

Figure 13 


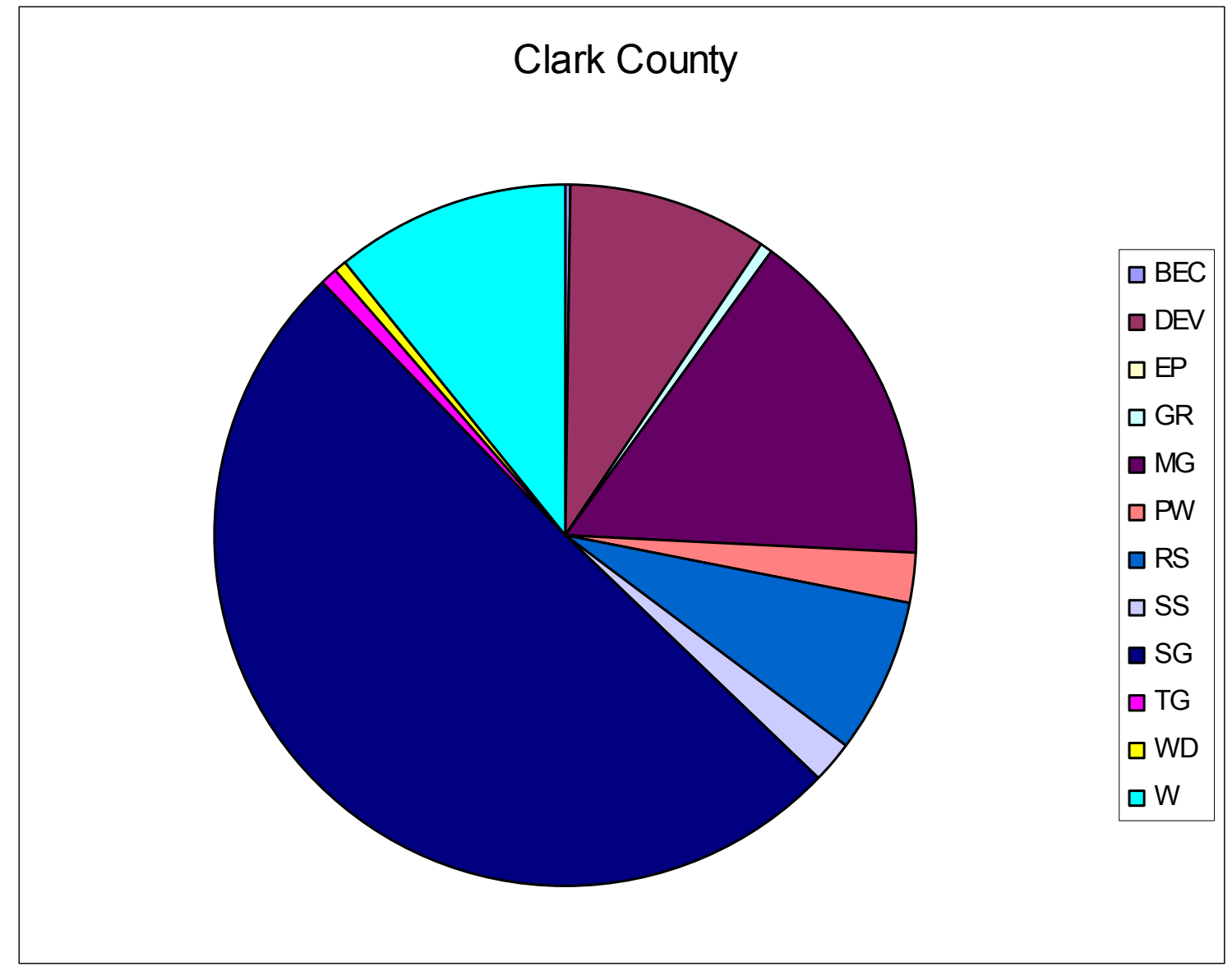

$\mathrm{BEC}=$ Bare Earth $/$ Construction

$\mathrm{DEV}=$ Developed

$\mathrm{EP}=$ Ephemeral Pool

$\mathrm{GR}=$ Gravel Road

$\mathrm{MG}=$ Medium Grass

$\mathrm{PW}=$ Permanent Water

$\mathrm{RS}=$ Runway System

$\mathrm{SS}=$ Scrub-Shrub

$\mathrm{SG}=$ Short Grass

$\mathrm{TG}=$ Tall Grass

$\mathrm{WD}=$ Weedy Ditch

$\mathrm{W}=$ Woodlot

Figure 14 


\section{Airport Habitat Map: DeKalb County}

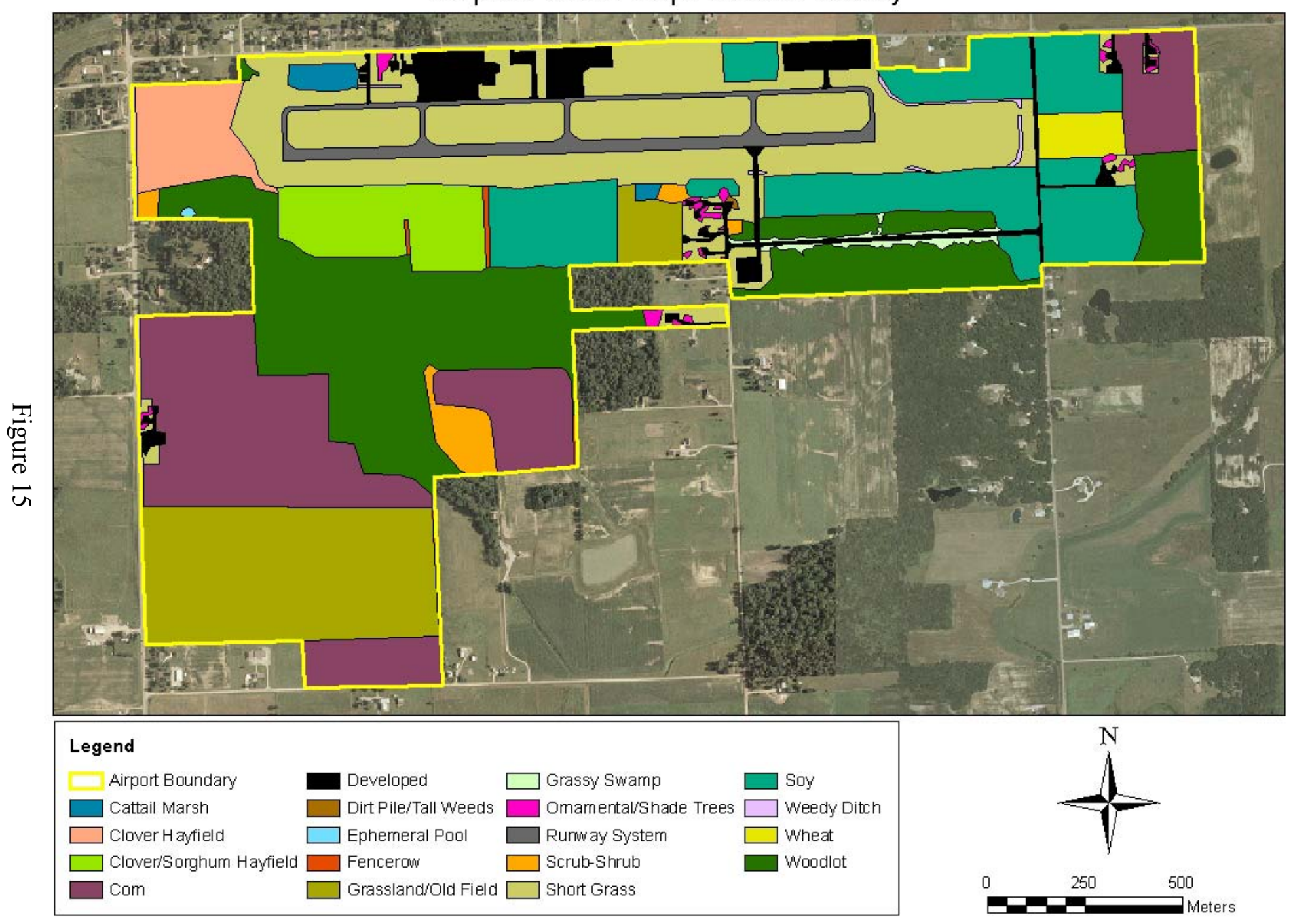




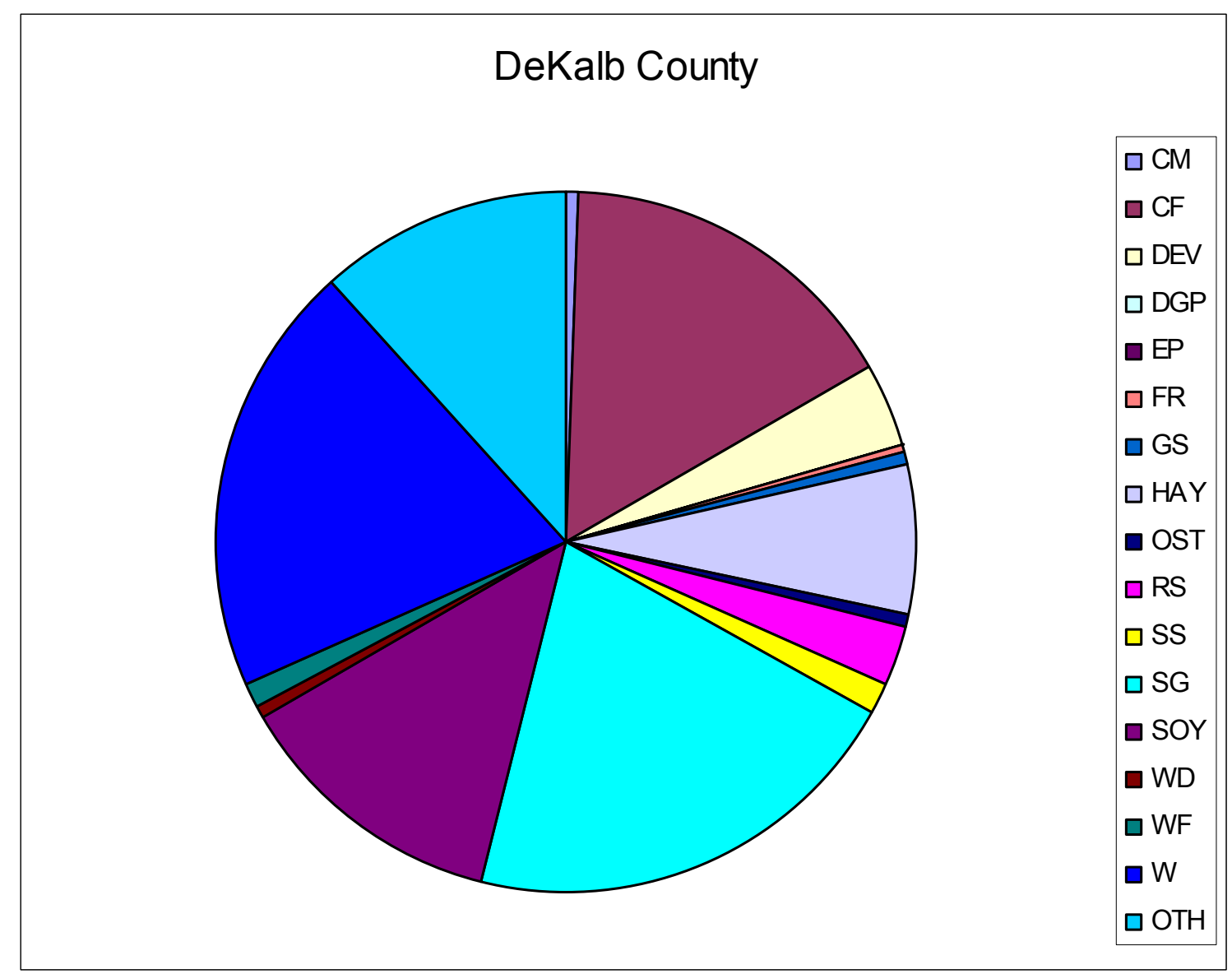

$\mathrm{CM}=$ Cattail Marsh

$\mathrm{CF}=$ Corn Field

$\mathrm{DEV}=$ Developed

DGP $=$ Dirt/Gravel Pile

$\mathrm{EP}=$ Ephemeral Pool

$\mathrm{FR}=$ Fencerow

GS = Grassy Swamp

HAY $=$ Hayfield

OST $=$ Ornamental/Shade Trees

$\mathrm{RS}=$ Runway System

$\mathrm{SS}=$ Scrub-Shrub

$\mathrm{SG}=$ Short Grass

SOY = Soybean Field

$\mathrm{WD}=$ Weedy Ditch

$\mathrm{WF}=$ Wheat Field

$\mathrm{W}=$ Woodlot

$\mathrm{OTH}=$ Other

Figure 16 
Airport Habitat Map: Greenwood Municipal

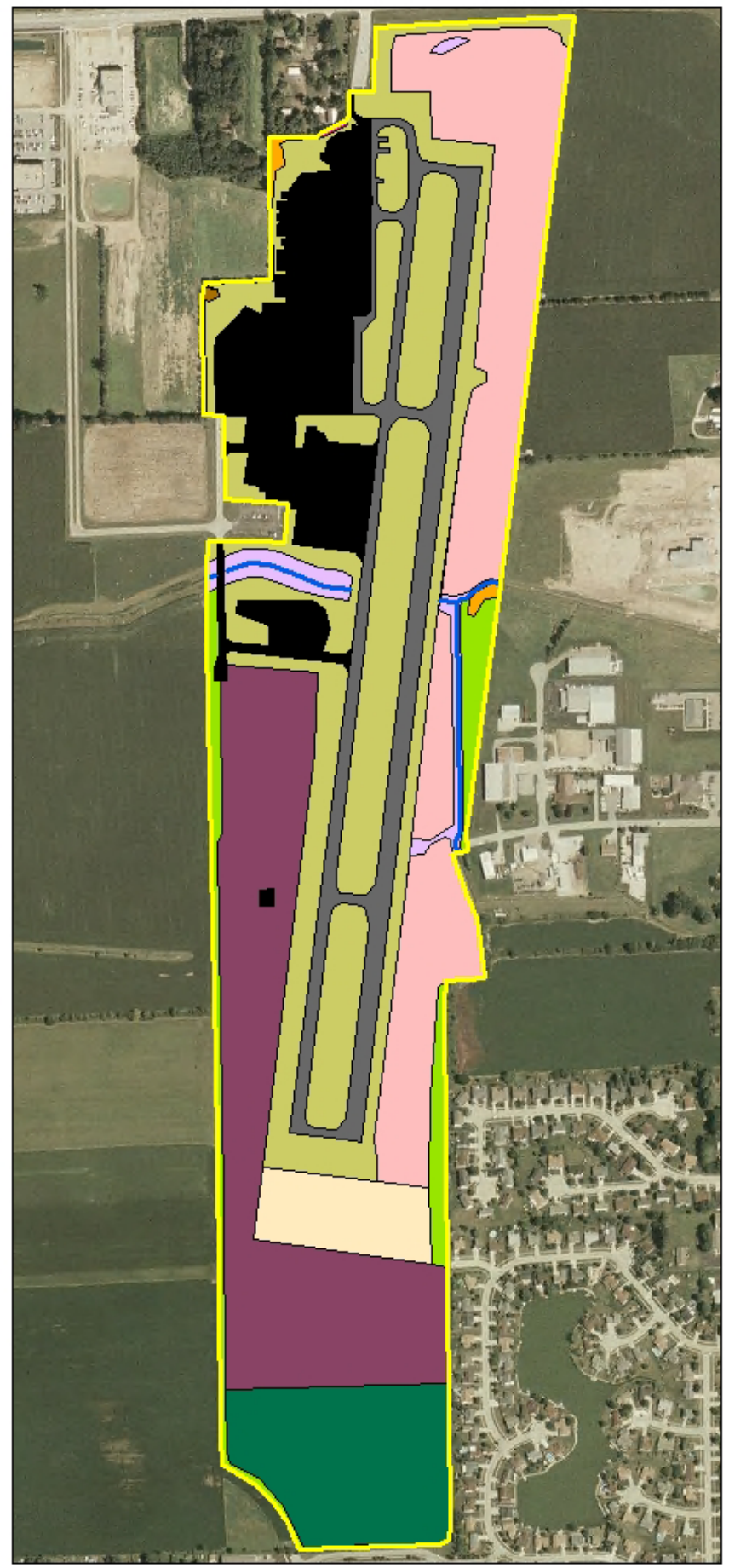

\begin{tabular}{|l|}
\hline Legend \\
Aiport Boundary \\
$\square$ Stream \\
Bare Earth/Construction \\
Corn \\
Developed \\
$\square$ Dirt Pile/Tall Weeds \\
$\square$ Hayfield \\
$\square$ Ornamental/Shade Trees \\
$\square$ Runway System \\
$\square$ Scrub-Shrub \\
$\square$ Short Grass \\
$\square$ Soy \\
$\square$ Tall Grass \\
$\square$ Meedy Ditch
\end{tabular}

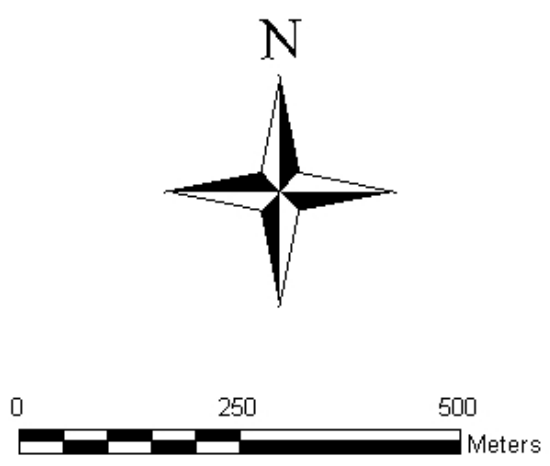

Figure 17 


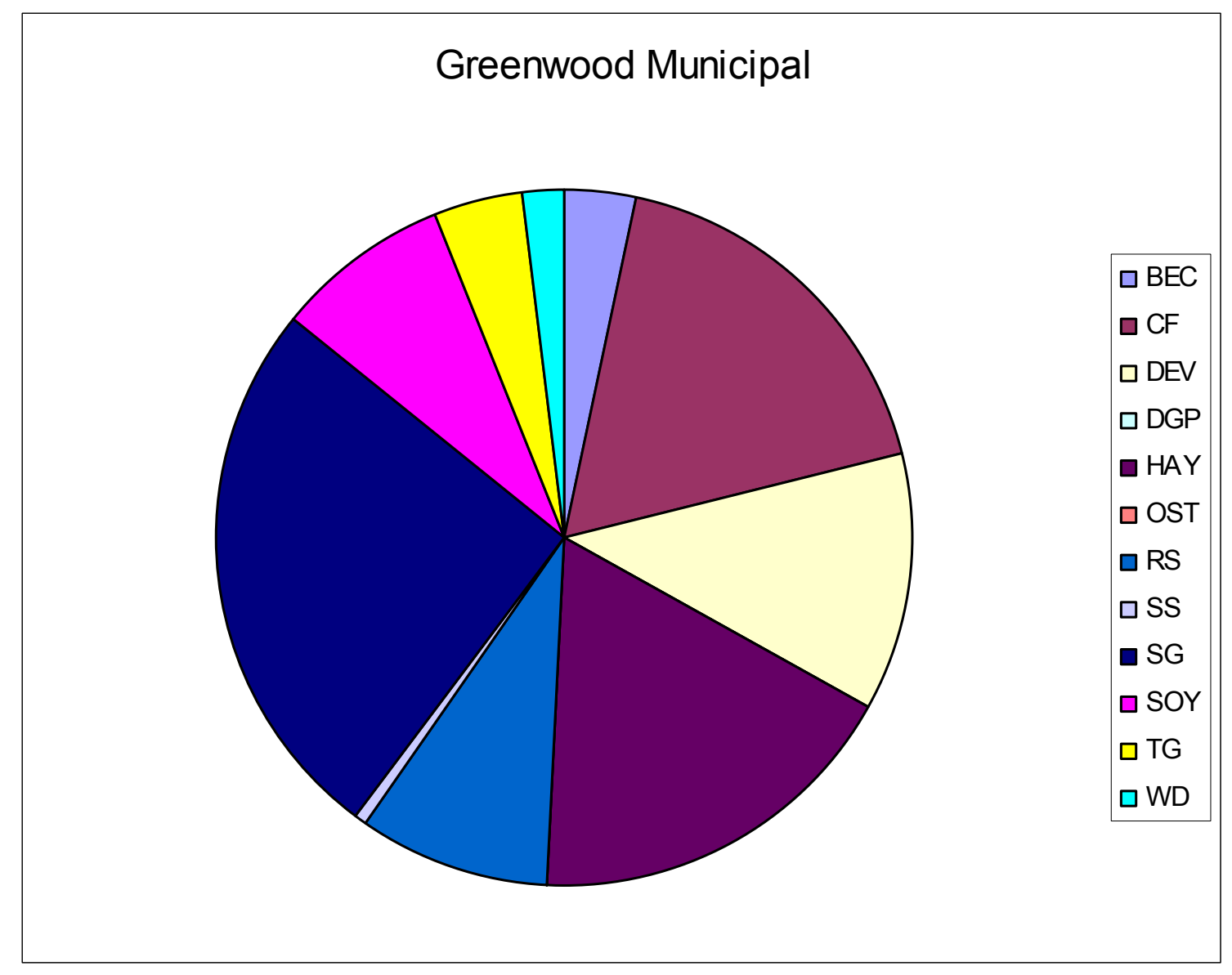

$\mathrm{BEC}=$ Bare Earth/Construction

$\mathrm{CF}=$ Corn Field

$\mathrm{DEV}=$ Developed

DGP $=$ Dirt/Gravel Pile

$\mathrm{HAY}=$ Hayfield

OST $=$ Ornamental/Shade Trees

$\mathrm{RS}=$ Runway System

$\mathrm{SS}=$ Scrub-Shrub

$\mathrm{SG}=$ Short Grass

SOY $=$ Soybean Field

$\mathrm{TG}=$ Tall Grass

$\mathrm{WD}=$ Weedy Ditch

Figure 18 


\section{Airport Habitat Map: Huntingburg}

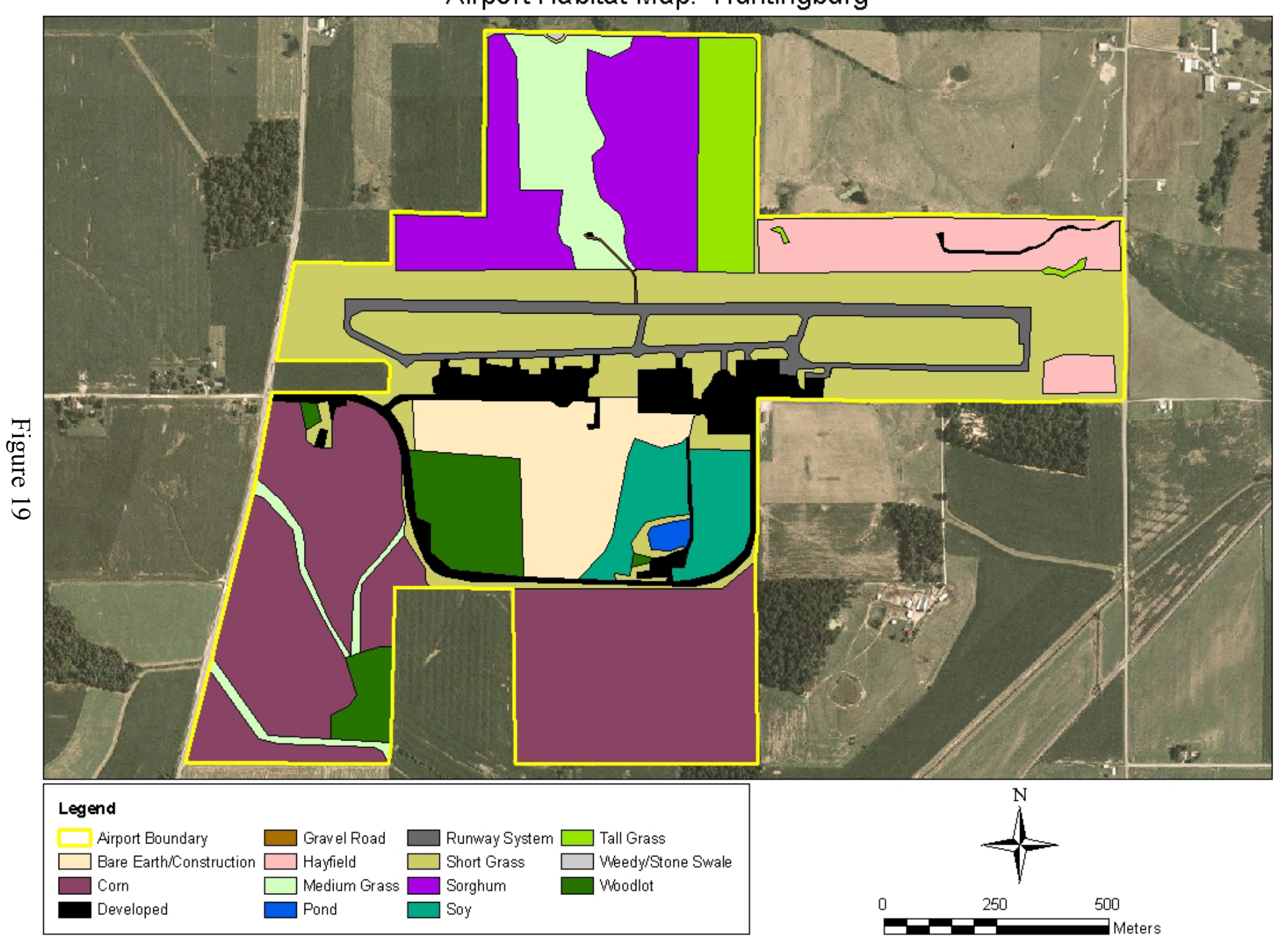




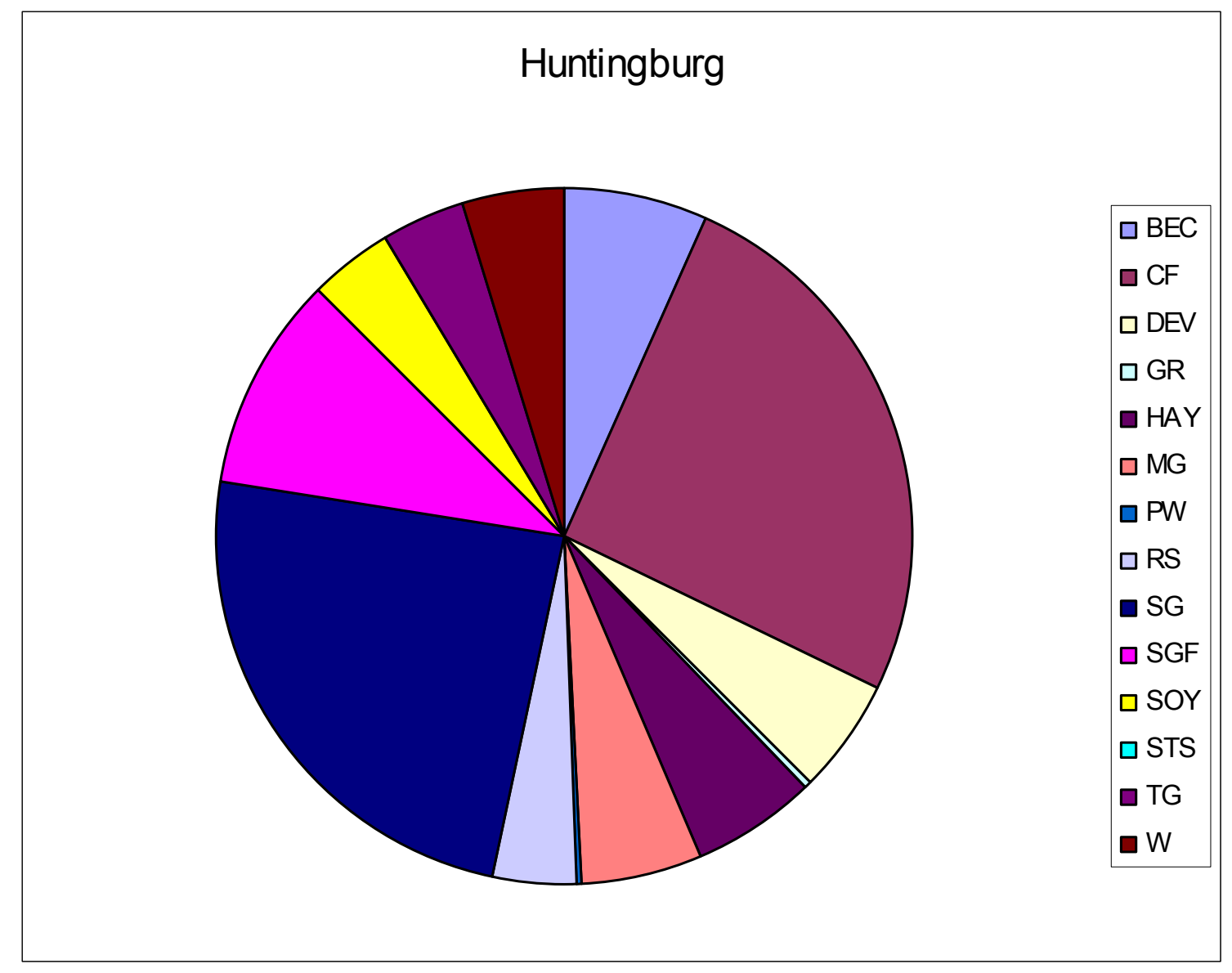

BEC = Bare Earth/Construction
CF $=$ Corn Field
DEV = Developed
GR = Gravel Road
HAY = Hayfield
MG = Medium Grass
PW = Permanent Water
RS = Runway System
SG = Short Grass
SGF = Sorghum Field
SOY = Soybean Field
STS = Stone Swale
TG = Tall Grass
W = Woodlot

Figure 20 
Airport Habitat Map: Purdue University

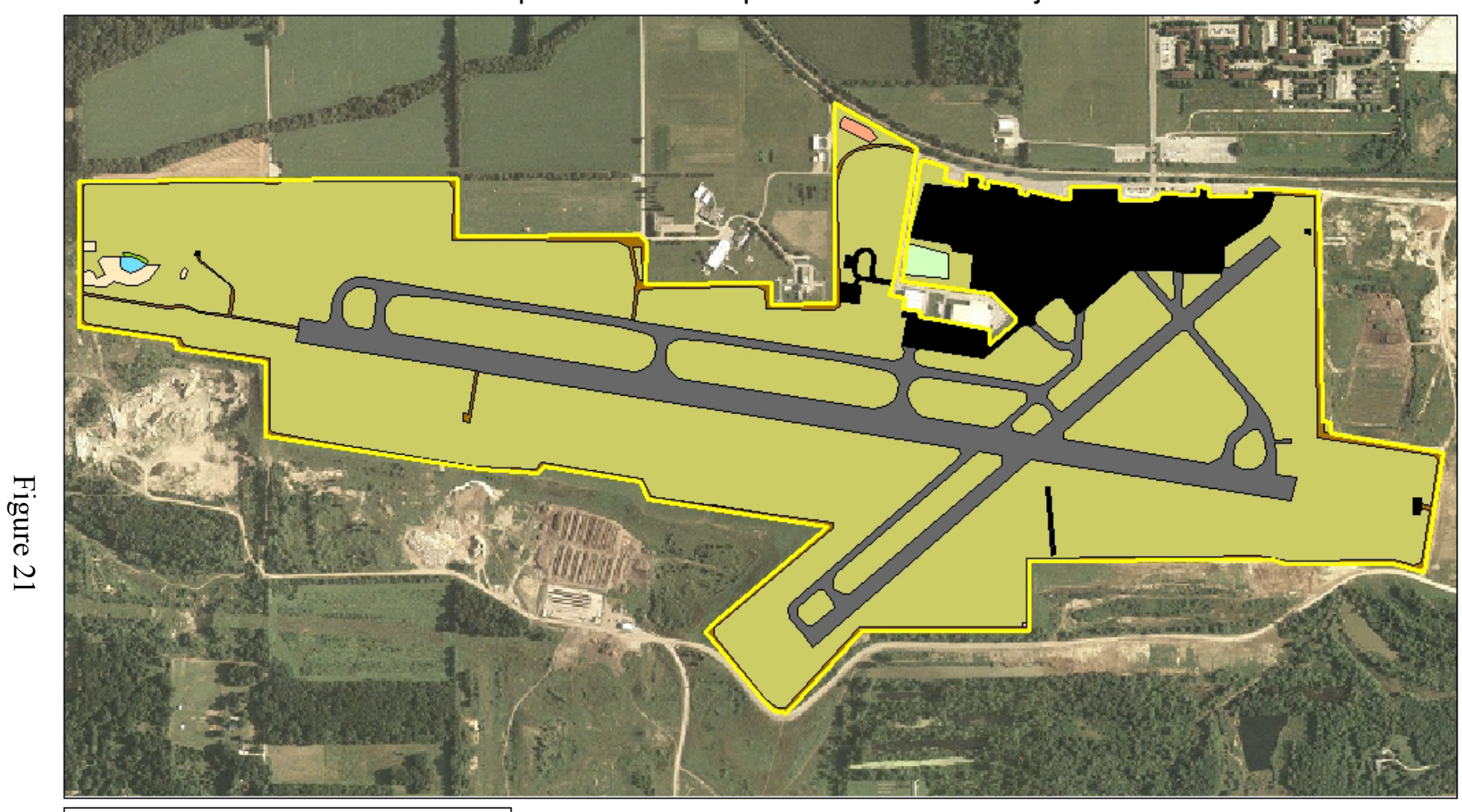

Legend

$\square$ Airport Boundary $\square$ Medium Grass

$\square$ Bare Earth $\square$ Permanent Water

Developed $\square$ Runway System

$\square$ Dirt Pile $\quad \square$ short Grass

$\square$ Ephemeral Pool $\square$ Tall Grass

$\square$ Gravel Road $\square$ Meedy Ditch

0

500

1,000

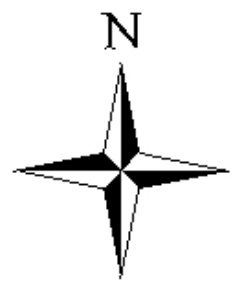




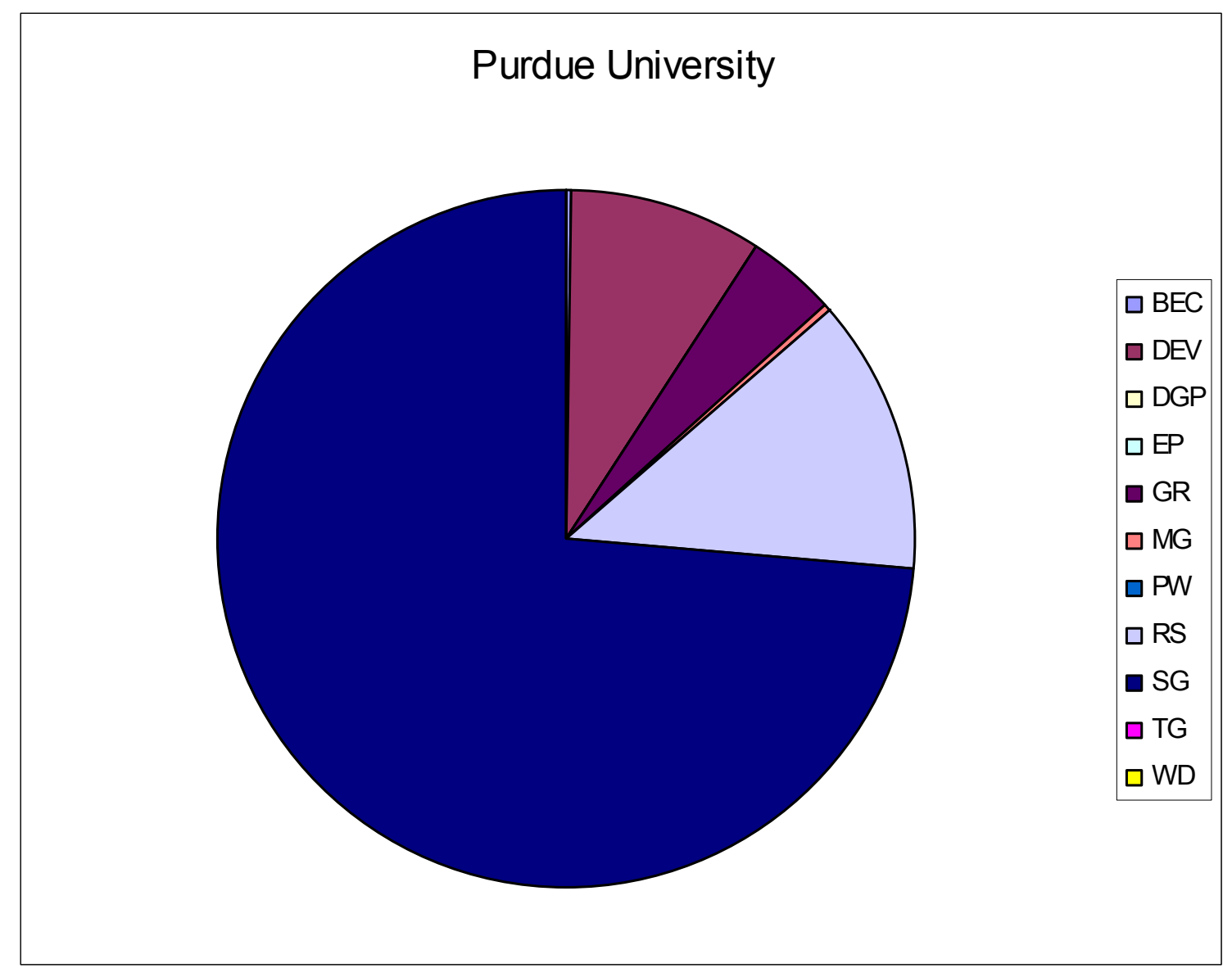

$\mathrm{BEC}=$ Bare Earth/Construction

$\mathrm{DEV}=$ Developed

DGP $=$ Dirt/Gravel Pile

$\mathrm{EP}=$ Ephemeral Pool

$\mathrm{GR}=$ Gravel Road

$\mathrm{MG}=$ Medium Grass

$\mathrm{PW}=$ Permanent Water

$\mathrm{RS}=$ Runway System

$\mathrm{SG}=$ Short Grass

$\mathrm{TG}=$ Tall Grass

$\mathrm{WD}=$ Weedy Ditch

Figure 22 


\section{Airport Habitat Map: Putnam County}
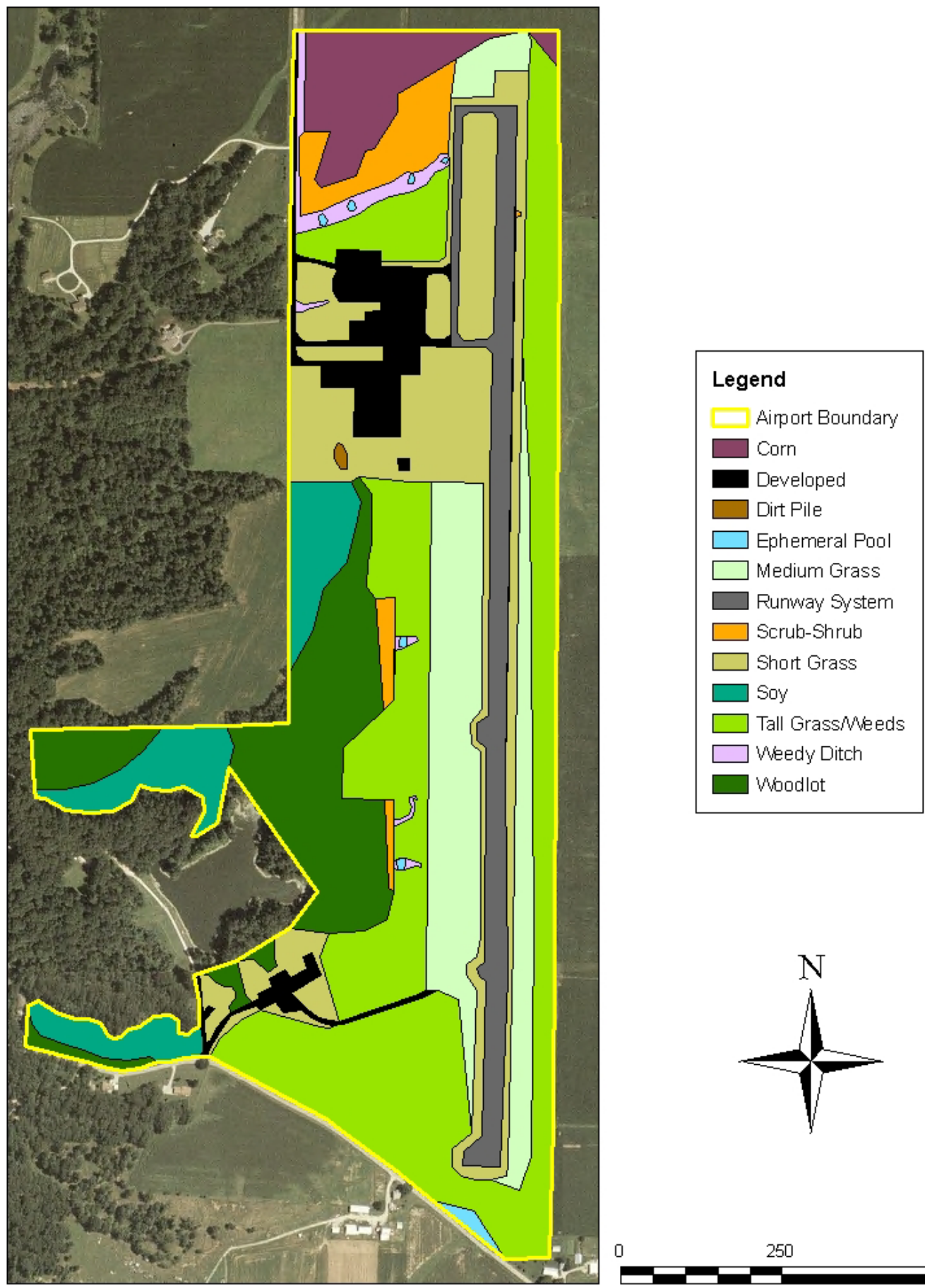

Figure 23
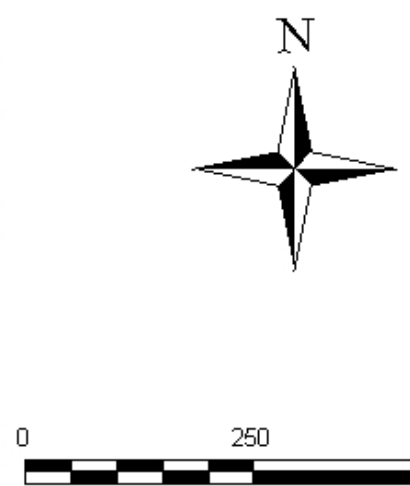


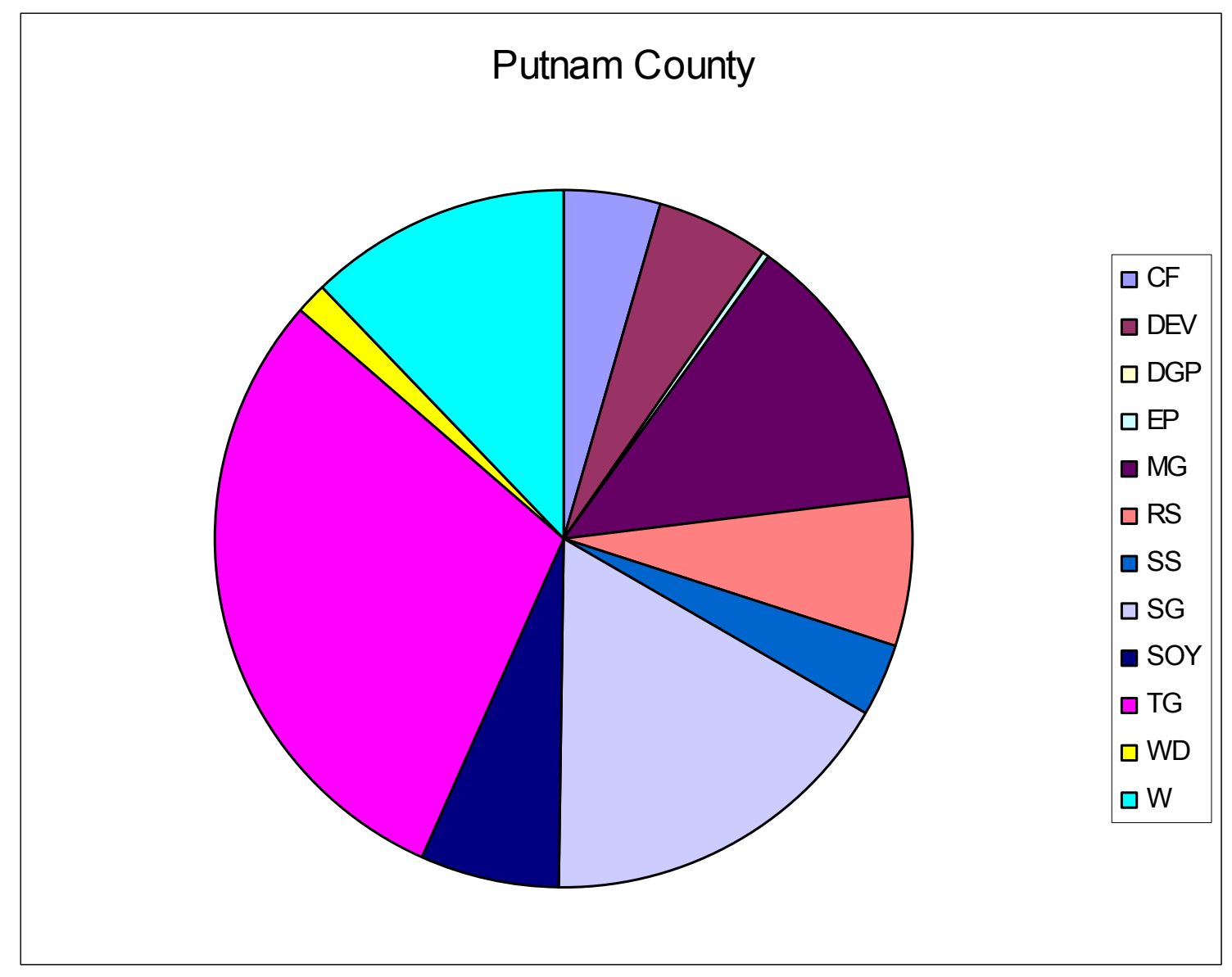

$$
\begin{aligned}
& \mathrm{CF}=\text { Corn Field } \\
& \mathrm{DEV}=\text { Developed } \\
& \text { DGP }=\text { Dirt } / \text { Gravel Pile } \\
& \mathrm{EP}=\text { Ephemeral Pool } \\
& \mathrm{MG}=\text { Medium Grass } \\
& \mathrm{RS}=\text { Runway System } \\
& \mathrm{SS}=\text { Scrub-Shrub } \\
& \mathrm{SG}=\text { Short Grass } \\
& \mathrm{SOY}=\text { Soybean Field } \\
& \mathrm{TG}=\text { Tall Grass } \\
& \mathrm{WD}=\text { Weedy Ditch } \\
& \mathrm{W}=\text { Woodlot }
\end{aligned}
$$

Figure 24 


\section{Airport Habitat Map: Richmond Municipal}

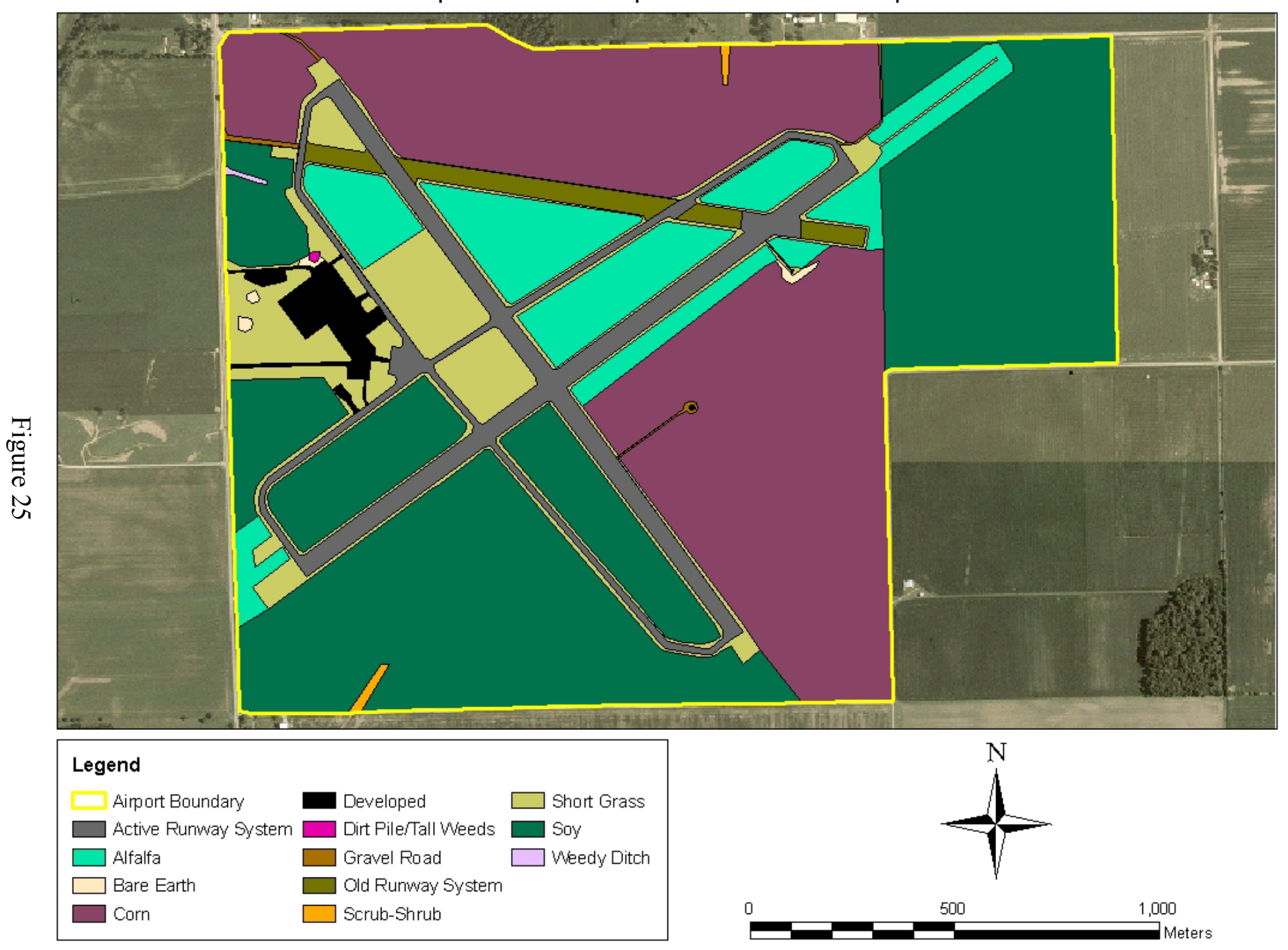




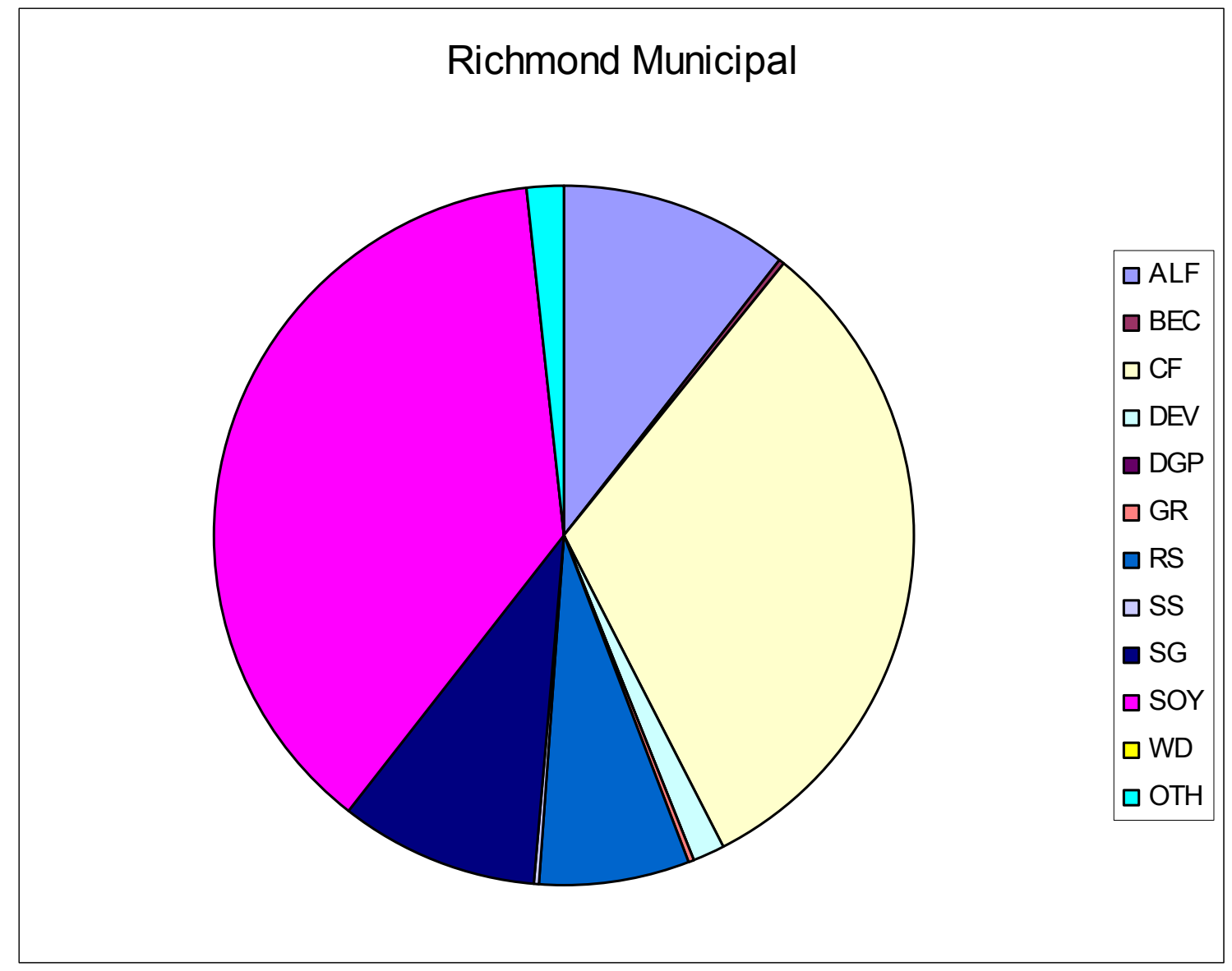

$\mathrm{ALF}=$ Alfalfa

$\mathrm{BEC}=$ Bare Earth/Construction

$\mathrm{CF}=$ Corn Field

$\mathrm{DEV}=$ Developed

DGP $=$ Dirt/Gravel Pile

GR $=$ Gravel Road

$\mathrm{RS}=$ Runway System

$\mathrm{SS}=$ Scrub-Shrub

$\mathrm{SG}=$ Short Grass

SOY $=$ Soybean Field

$\mathrm{WD}=$ Weedy Ditch

$\mathrm{OTH}=$ Other

Figure 26 
Airport Habitat Map: South Bend Regional

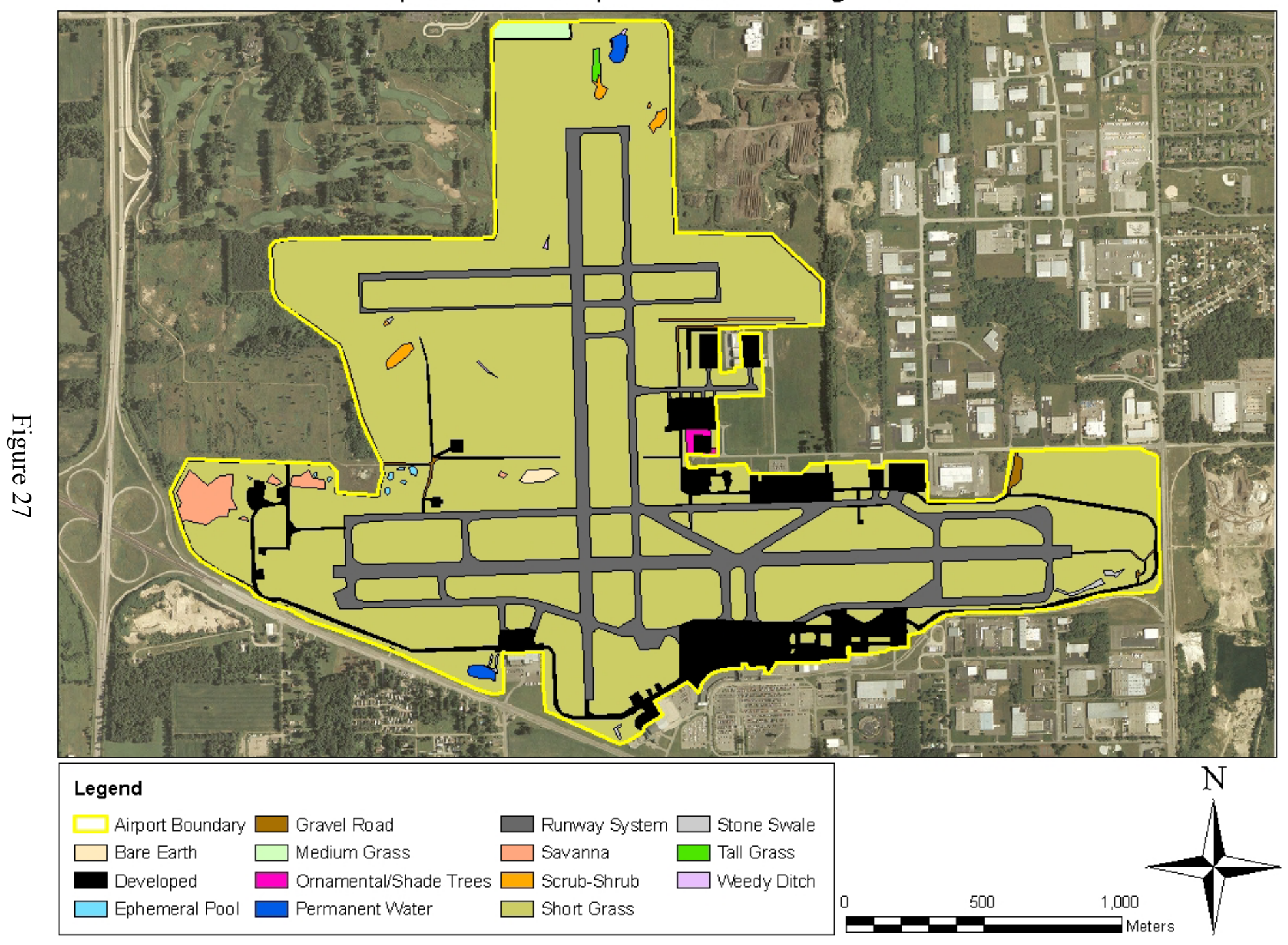




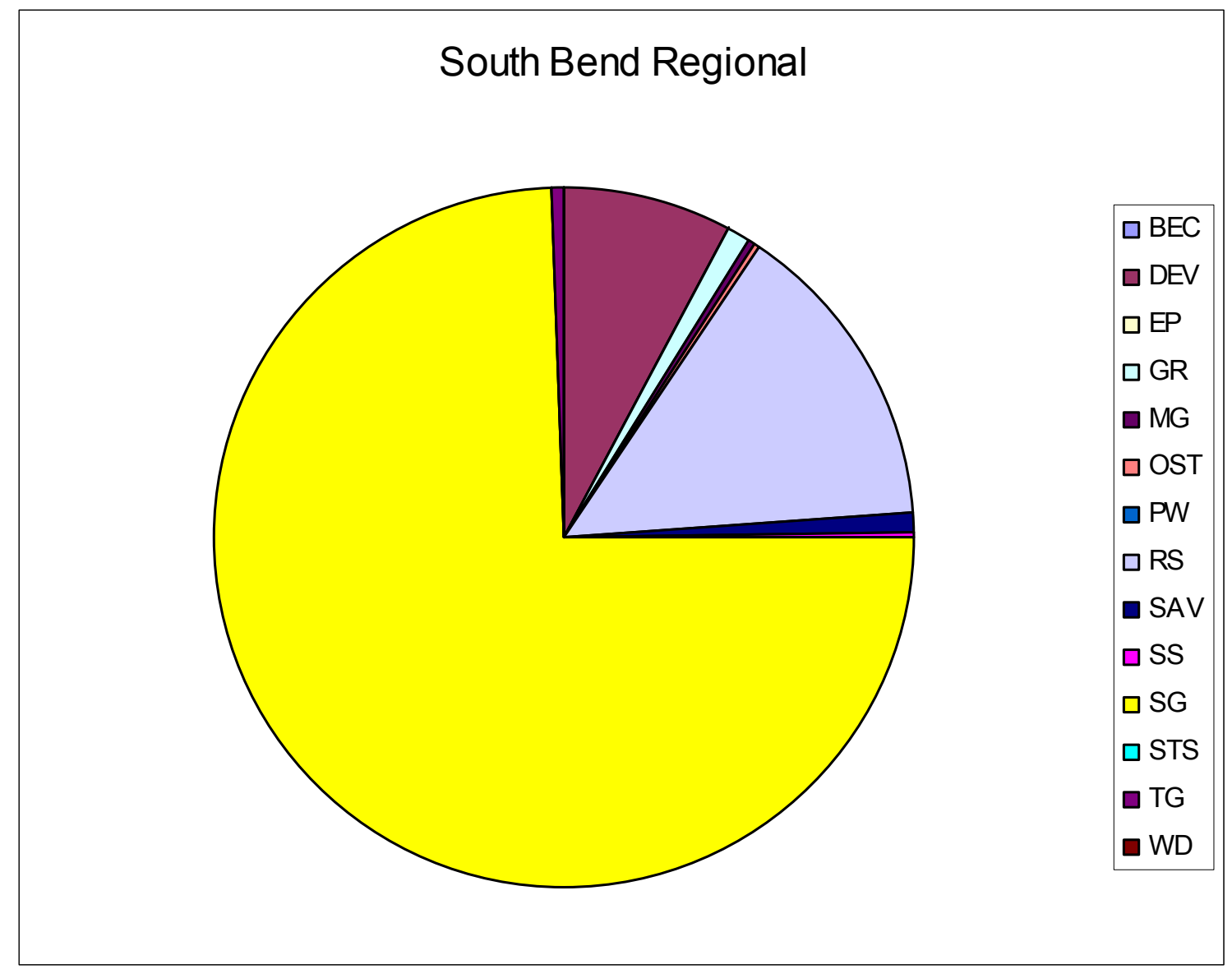

BEC = Bare Earth/Construction
DEV = Developed
EP = Ephemeral Pool
GR = Gravel Road
MG = Medium Grass
OST = Ornamental/Shade Trees
PW = Permanent Water
RS = Runway System
SAV = Savanna
SS = Scrub-Shrub
SG = Short Grass
STS = Stone Swale
TG = Tall Grass
WD = Weedy Ditch

Figure 28 
Airport Habitat Map: Warsaw Municipal

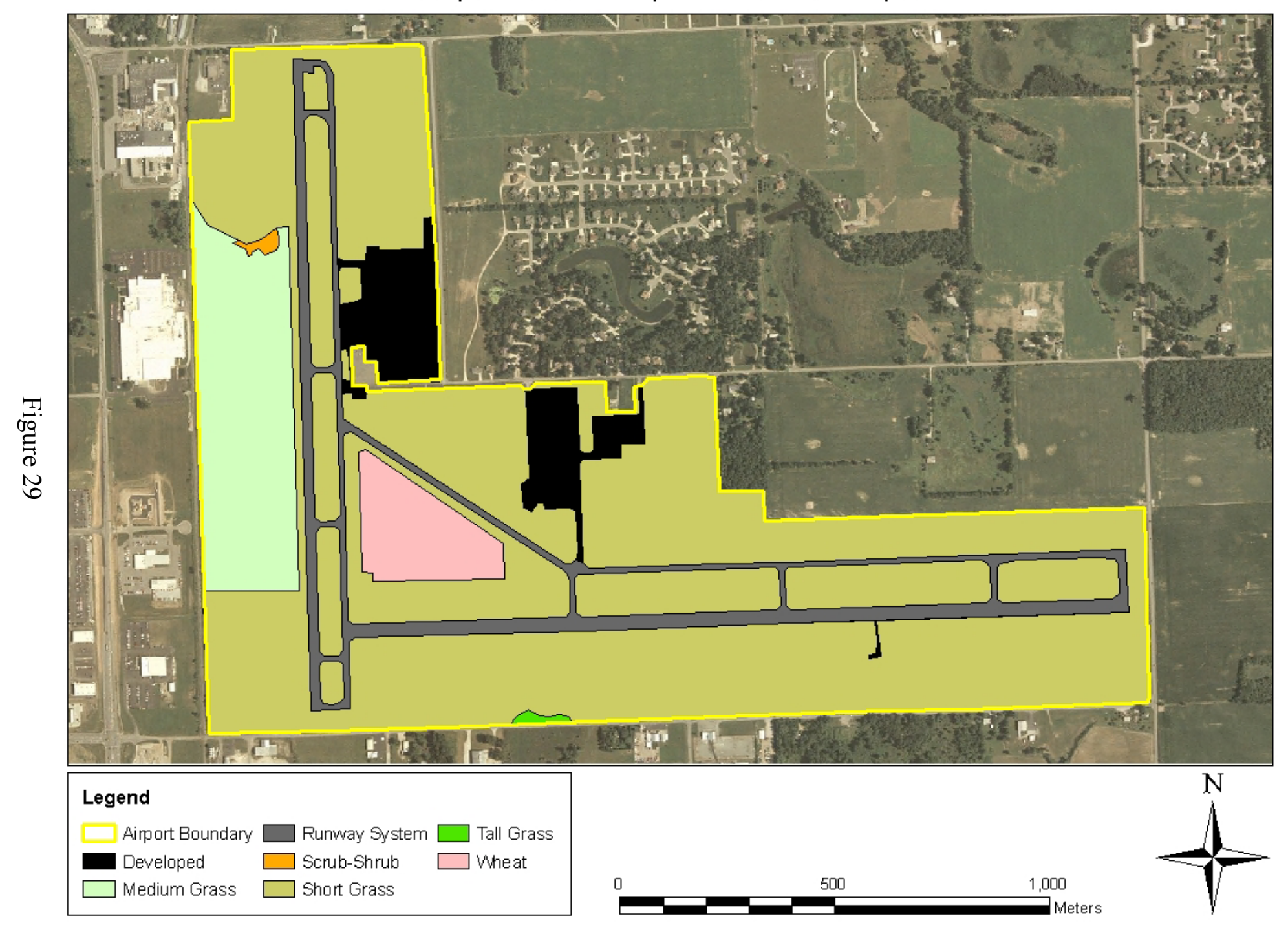




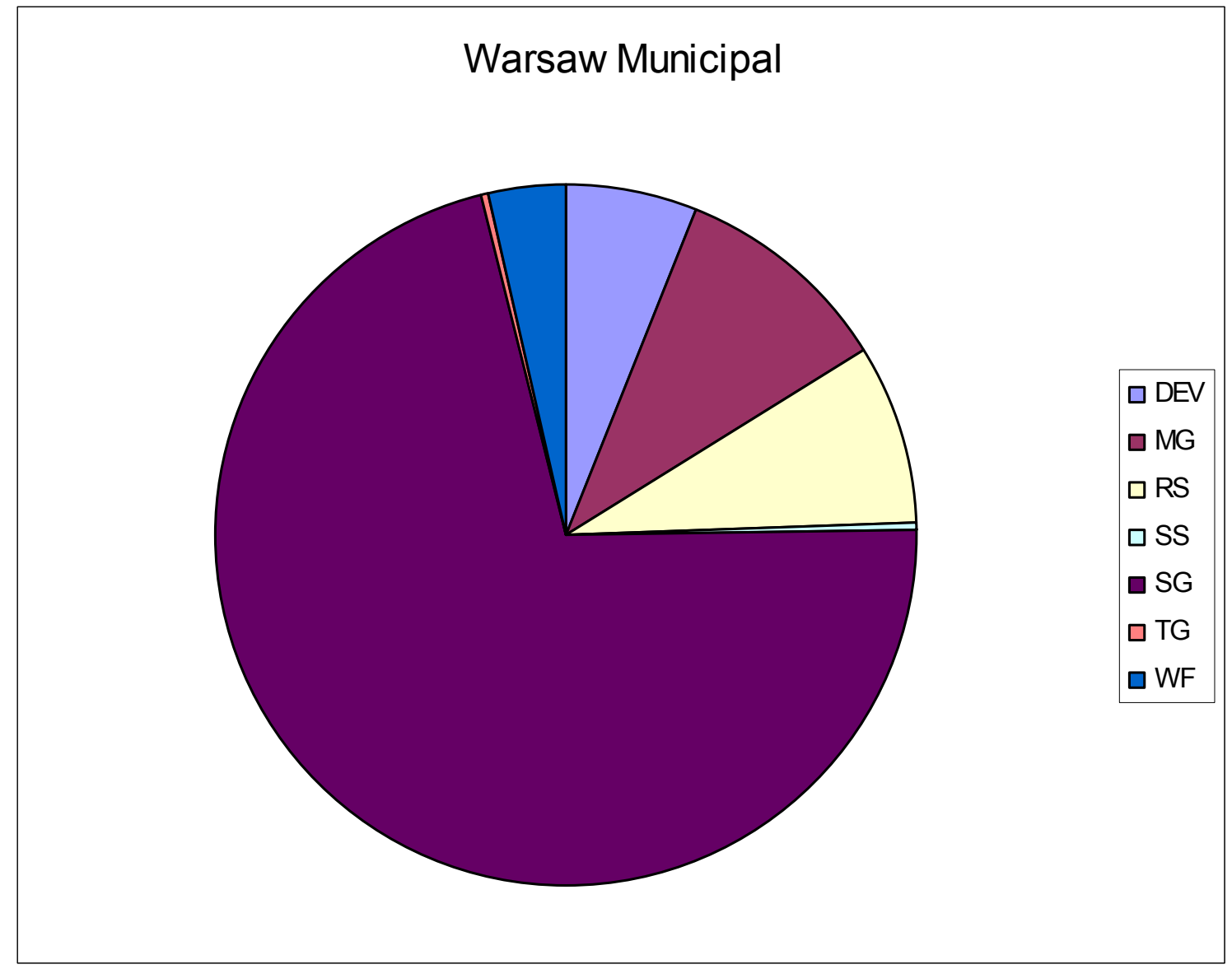

$\mathrm{DEV}=$ Developed

$\mathrm{MG}=$ Medium Grass

$\mathrm{RS}=$ Runway System

$\mathrm{SS}=$ Scrub-Shrub

$\mathrm{SG}=$ Short Grass

$\mathrm{TG}=$ Tall Grass

$\mathrm{WF}=$ Wheat Field

Figure 30 
Figures 31-40.

Types of fencing and locations of fence openings observed at 10 airports as part of the evaluation of wildlife hazards at general aviation airports in Indiana, 2005-2006. Fence openings were assessed only when chain-link fencing enclosed $\geq 25 \%$ of the airport perimeter.

\section{Fence Types}

Type A: $305-\mathrm{cm}$ (120-in) chain-link, 3 strands of barbed wire on top

Type B: 244-cm (96-in) chain-link, 3 strands of barbed wire on top

Type C: $213-$ to $244-\mathrm{cm}$ (84- to 96-in) chain-link

Type D: 213-cm (84-in) chain-link, 3 strands of barbed wire on top

Type E: $213-\mathrm{cm}(84-\mathrm{in})$ chain-link

Type F: 183 - to $213-\mathrm{cm}$ (72- to 84 -in) chain-link, plus $30-61 \mathrm{~cm}(12-24$ in) buried

Type G: $183-\mathrm{cm}$ (72-in) chain-link, 3 strands of barbed wire on top

Type H: $183-\mathrm{cm}$ (72-in) chain-link

Type I: 91 - to $137-\mathrm{cm}$ (36- to 54-in) chain-link

Type J: Other: $213-\mathrm{cm}(84-\mathrm{in})$ plastic mesh (5-cm [2-in] squares); $183-\mathrm{cm}$ (72-in) wood-panel; 91- to 137-cm (36- to 54-in) wire mesh (15-cm [6-in] squares); 5 strands barbed wire (137 cm [54 in] tall)

\section{Fence Openings}

Break: Opening between two segments of a fenceline (e.g., where a driveway or pedestrian corridor occurred)

Culvert: Open culvert underneath fence Dig-hole: Hole excavated underneath fence

Gap: Open space between bottom of fence and the ground, or between doors of a gate in the fenceline

Hole: Missing portion of a fence created by chewing/gnawing or other destructive action

Warp: Open space between bottom of fence and the ground, caused by warping or other physical damage to bottom of fence

Other: Actions outside the fenceline that have essentially eliminated effectiveness of the fence in preventing larger mammals from jumping over it (e.g, by raising the height of a road or filling a ditch with gravel) 
Airport Fencing Map: Anderson Municipal

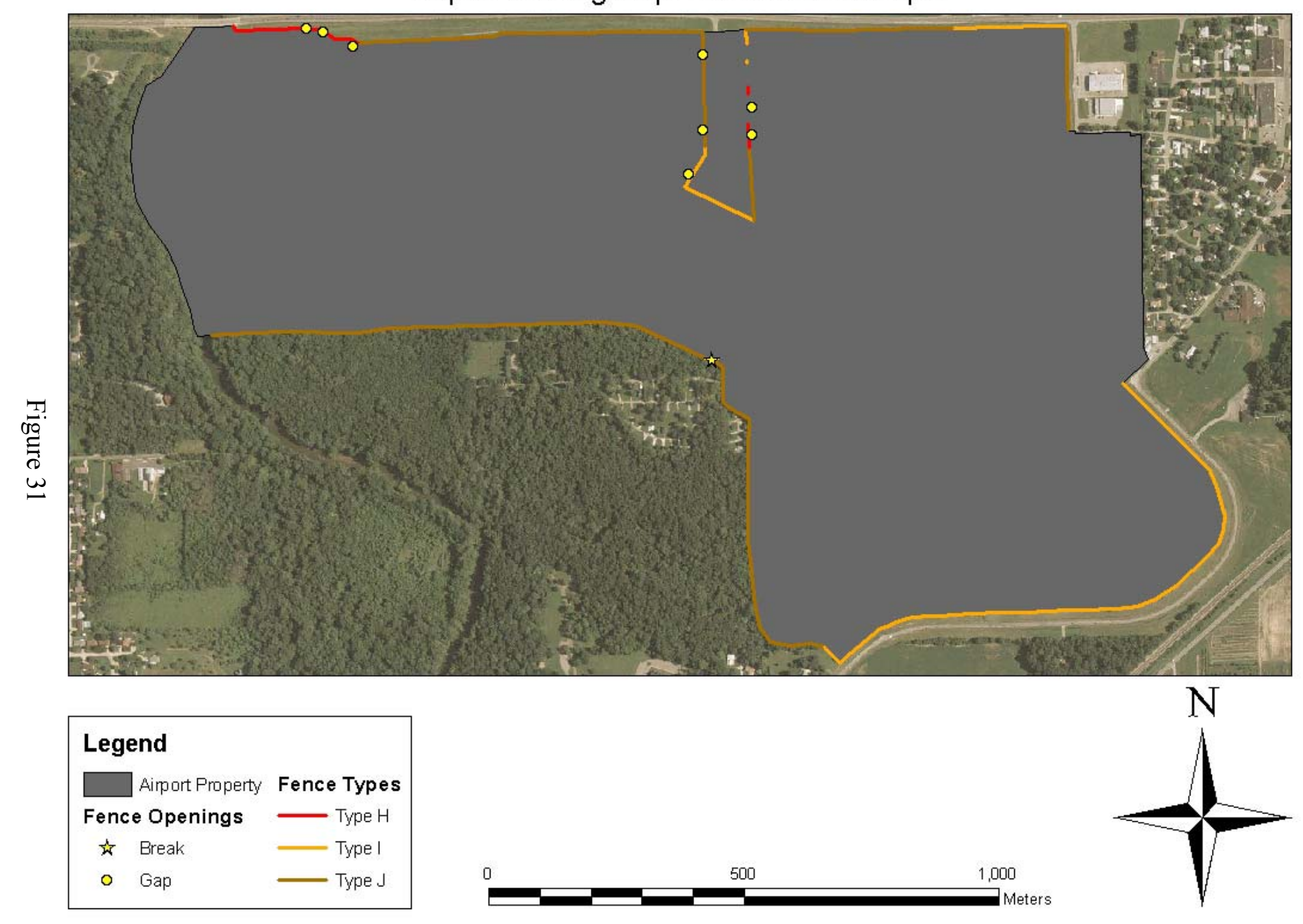




\section{Airport Fencing Map: Clark County}

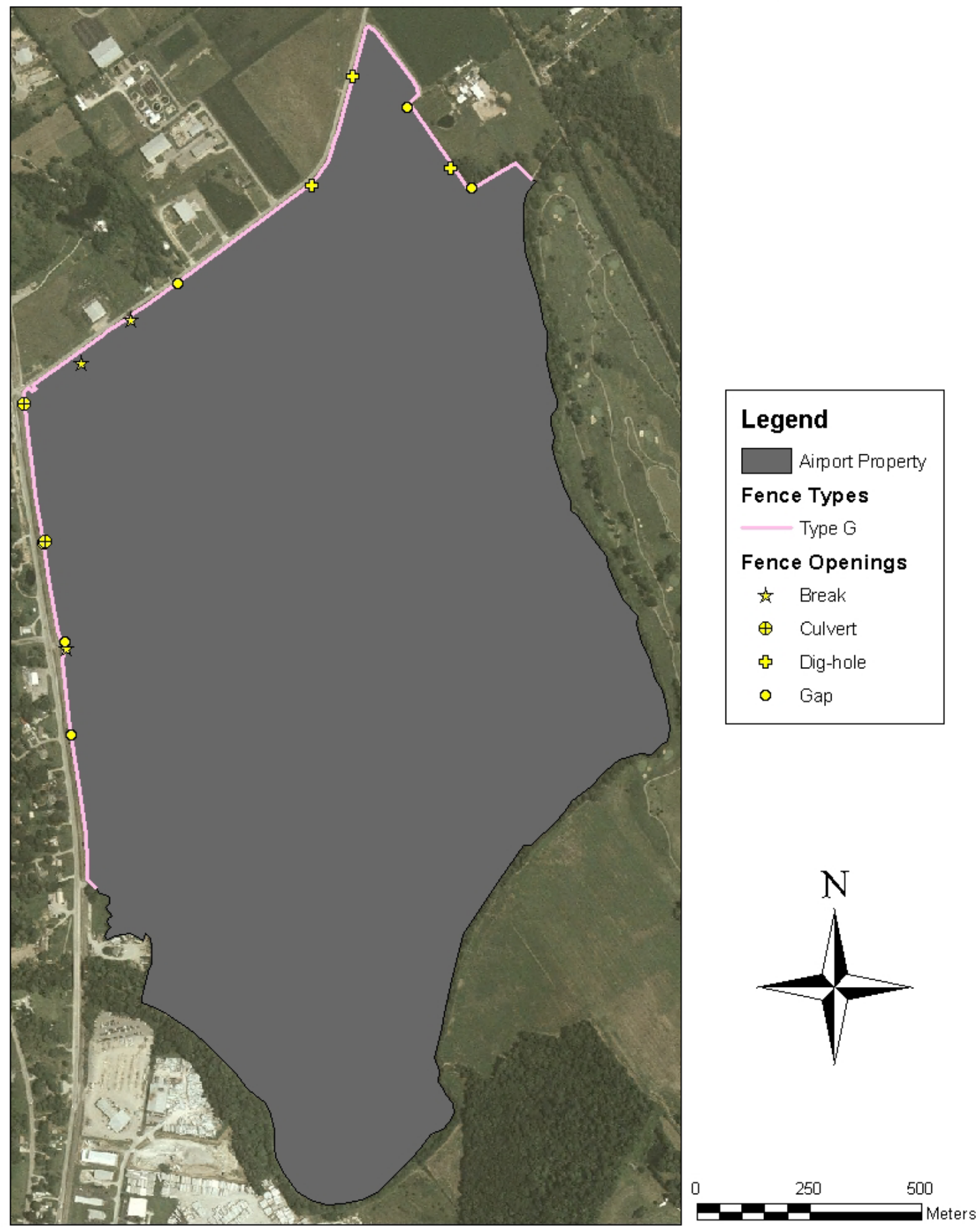

Figure 32 


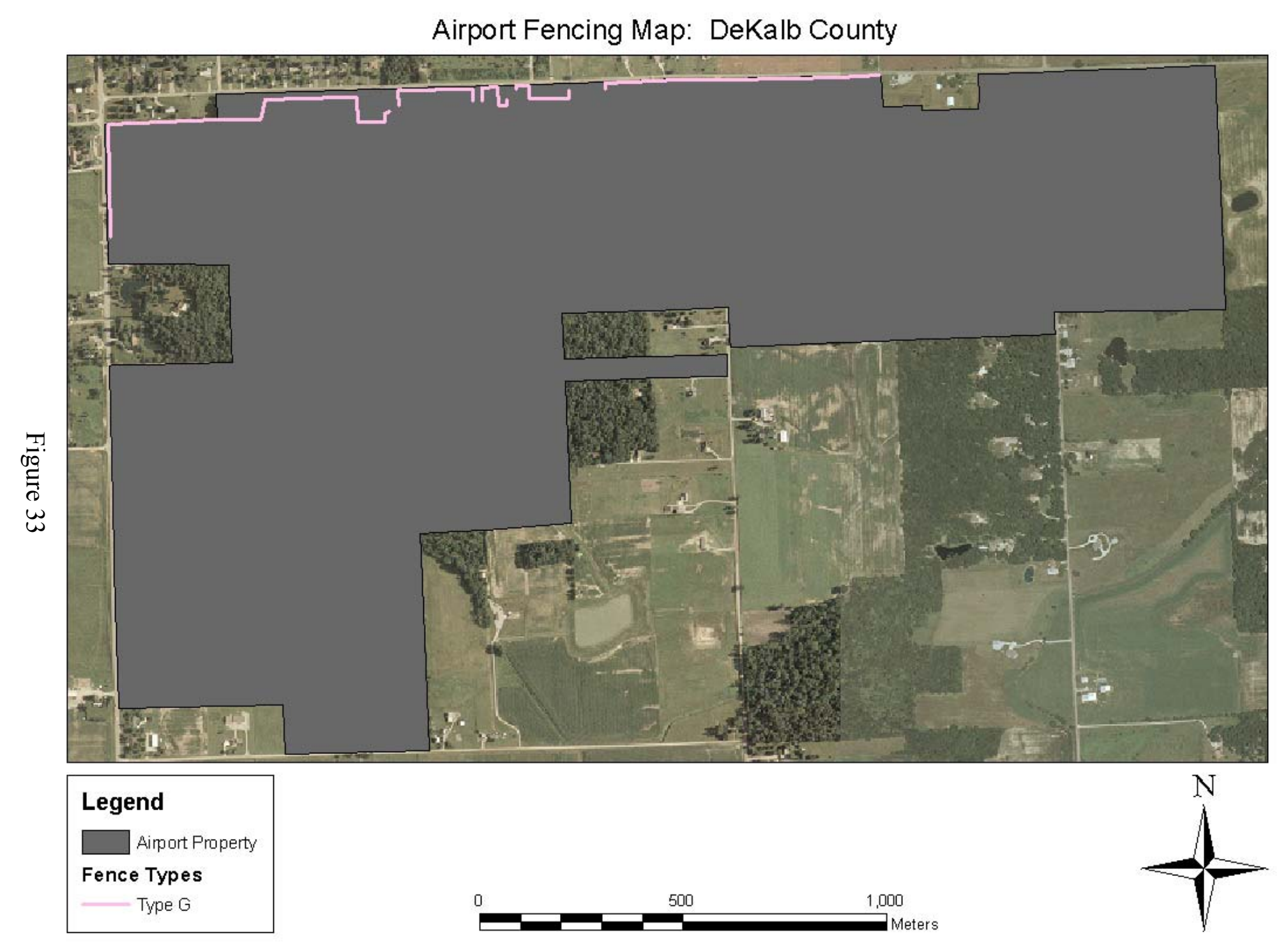


Airport Fencing Map: Greenwood Municipal

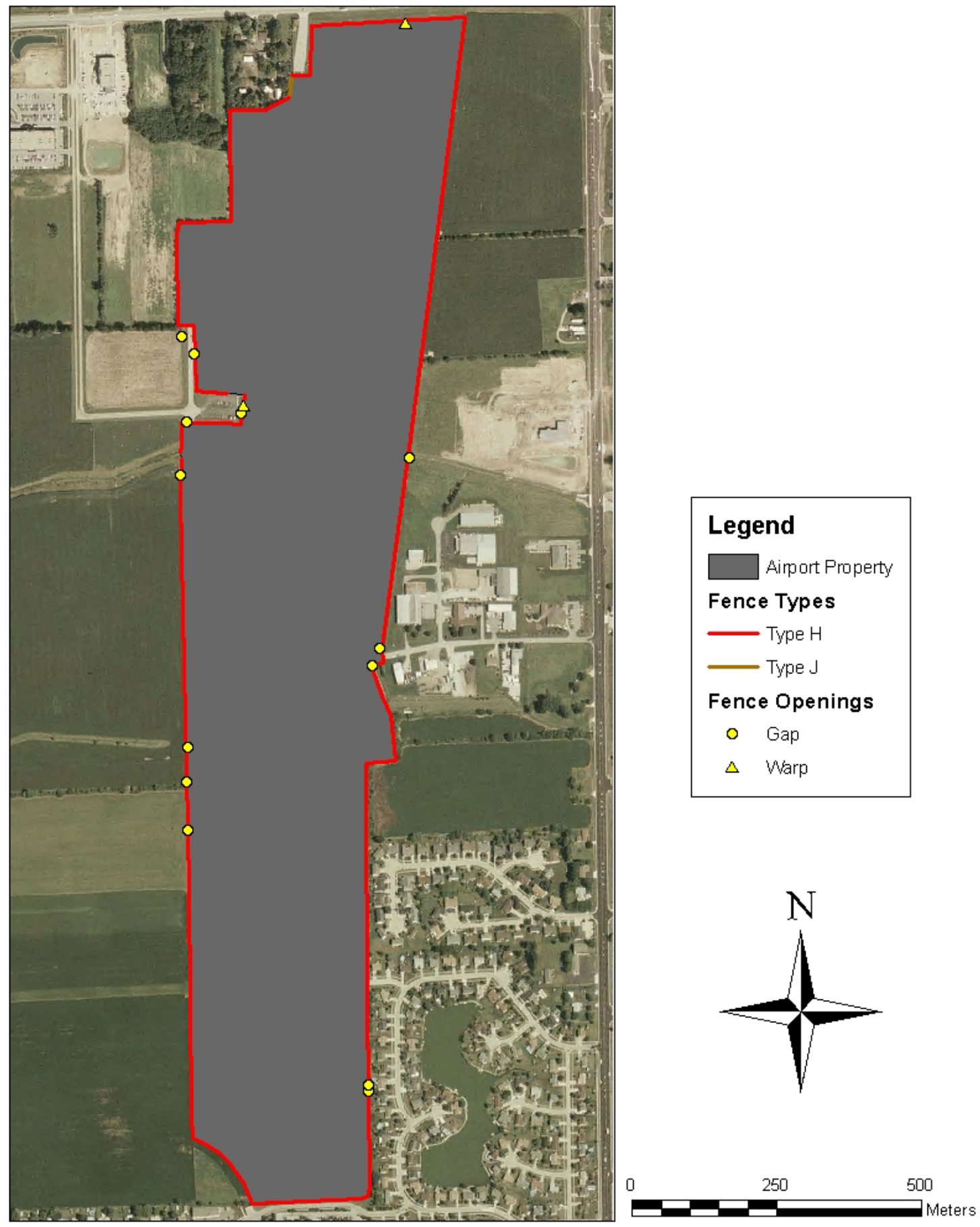

Figure 34 
Airport Fencing Map: Huntingburg

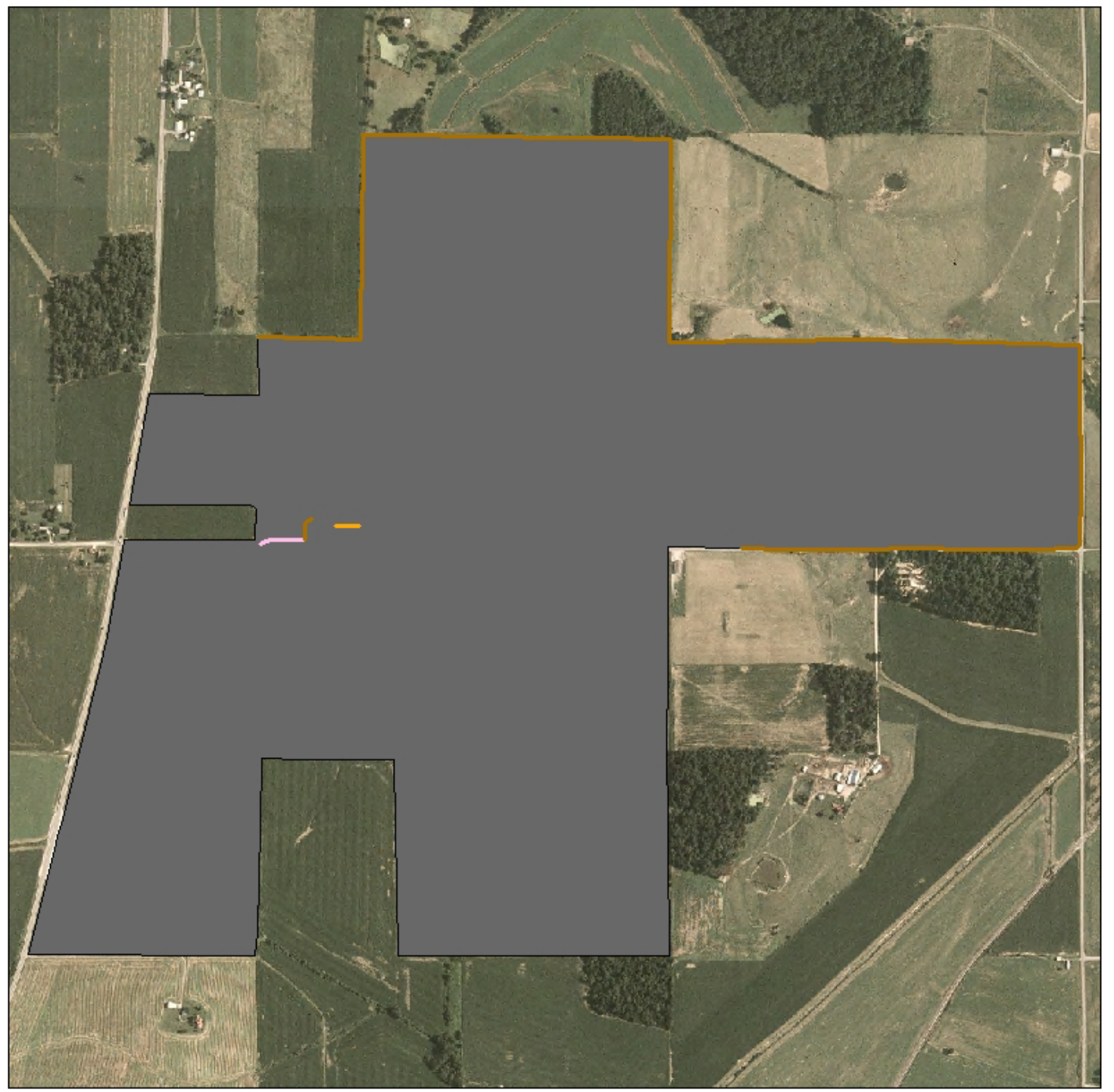

\begin{tabular}{|l|}
\hline Legend \\
$\begin{array}{l}\text { Aipport Property } \\
\text { Fence Types } \\
\text { Type G } \\
\text { Type I } \\
\text { Type J }\end{array}$ \\
\hline
\end{tabular}

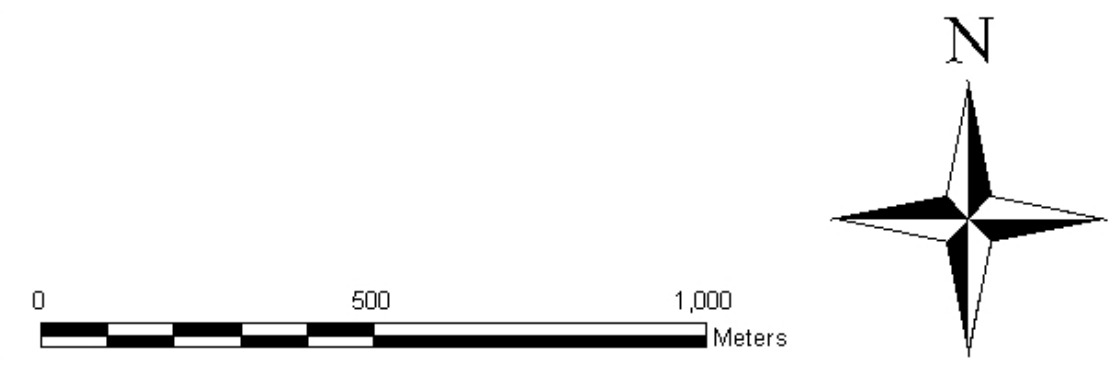

Figure 35 


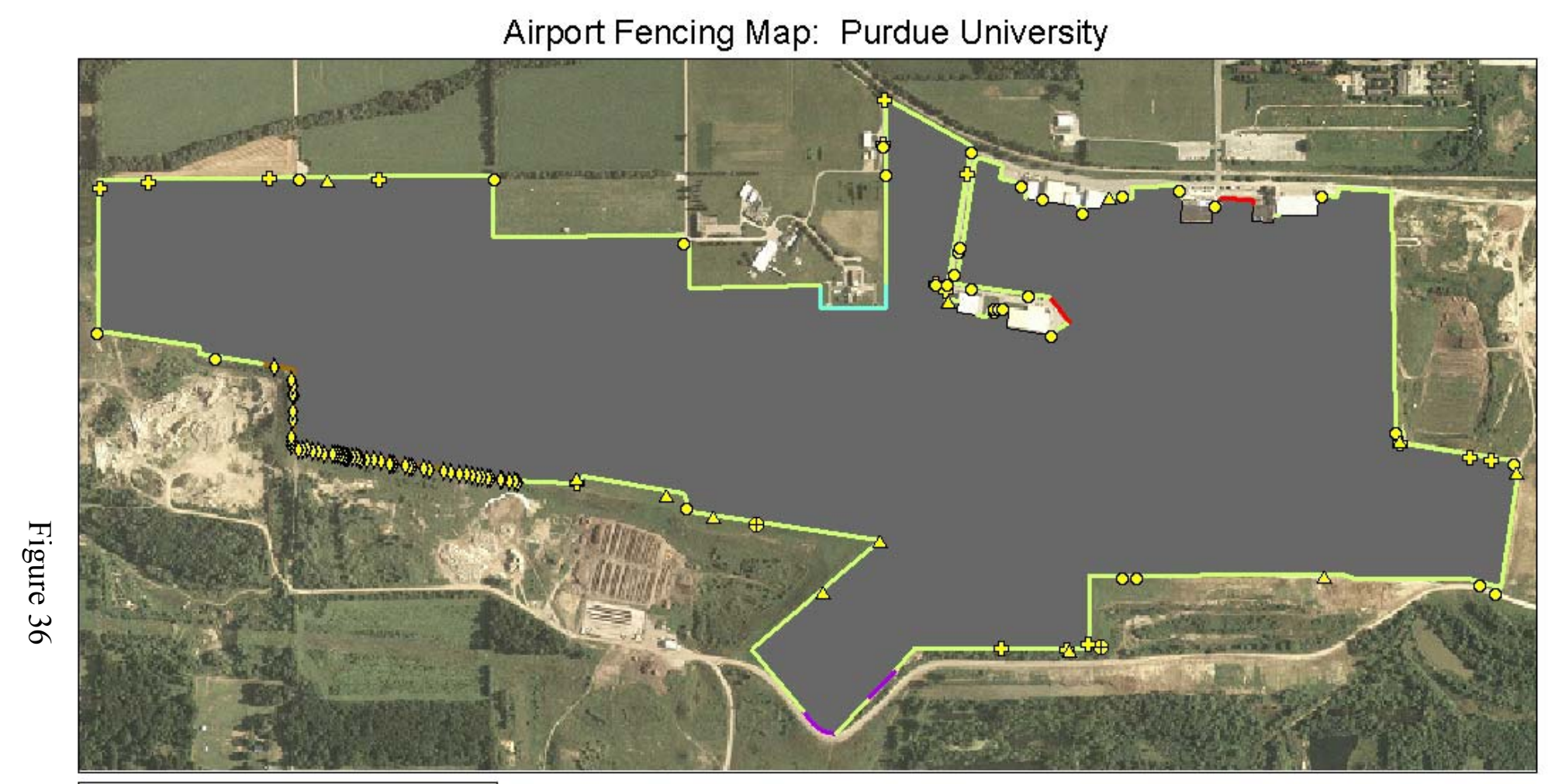

\begin{tabular}{|c|c|c|}
\hline \multicolumn{3}{|c|}{ Legend } \\
\hline & Aiport Property & Fence Types \\
\hline \multicolumn{2}{|c|}{ Fence Openings } & Type C \\
\hline$\oplus$ & Culvert & — Type D \\
\hline$\xi$ & Dig-hole & — Type E \\
\hline 0 & Gap & — Type F \\
\hline$\theta$ & Hole & — Type H \\
\hline$\Delta$ & Warp & — Type J \\
\hline
\end{tabular}
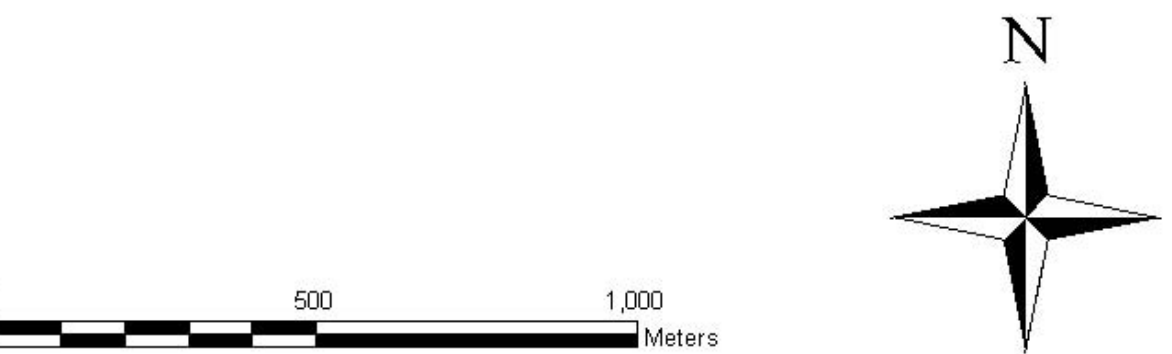


\section{Airport Fencing Map: Putnam County}

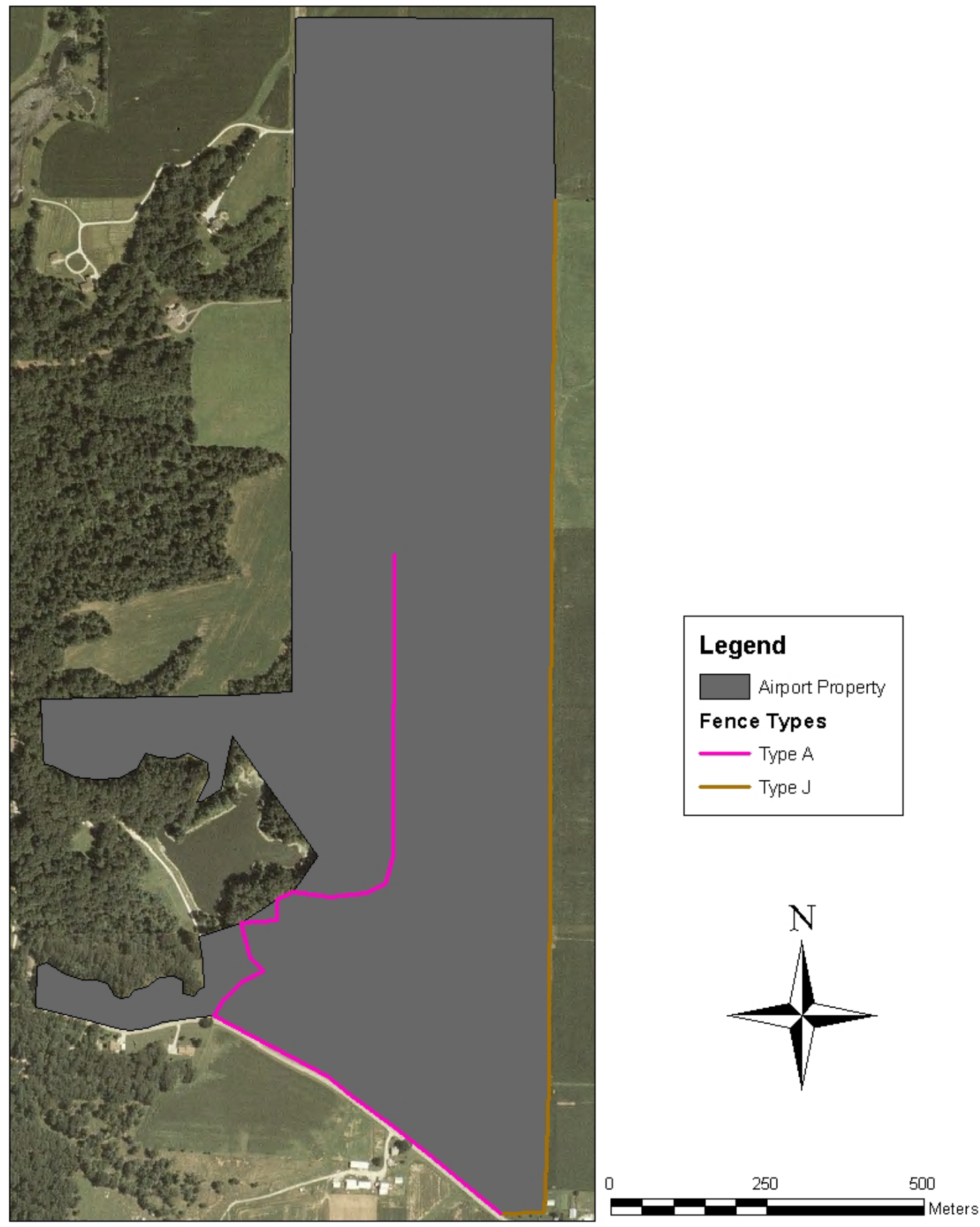

Figure 37 
Airport Fencing Map: Richmond Municipal

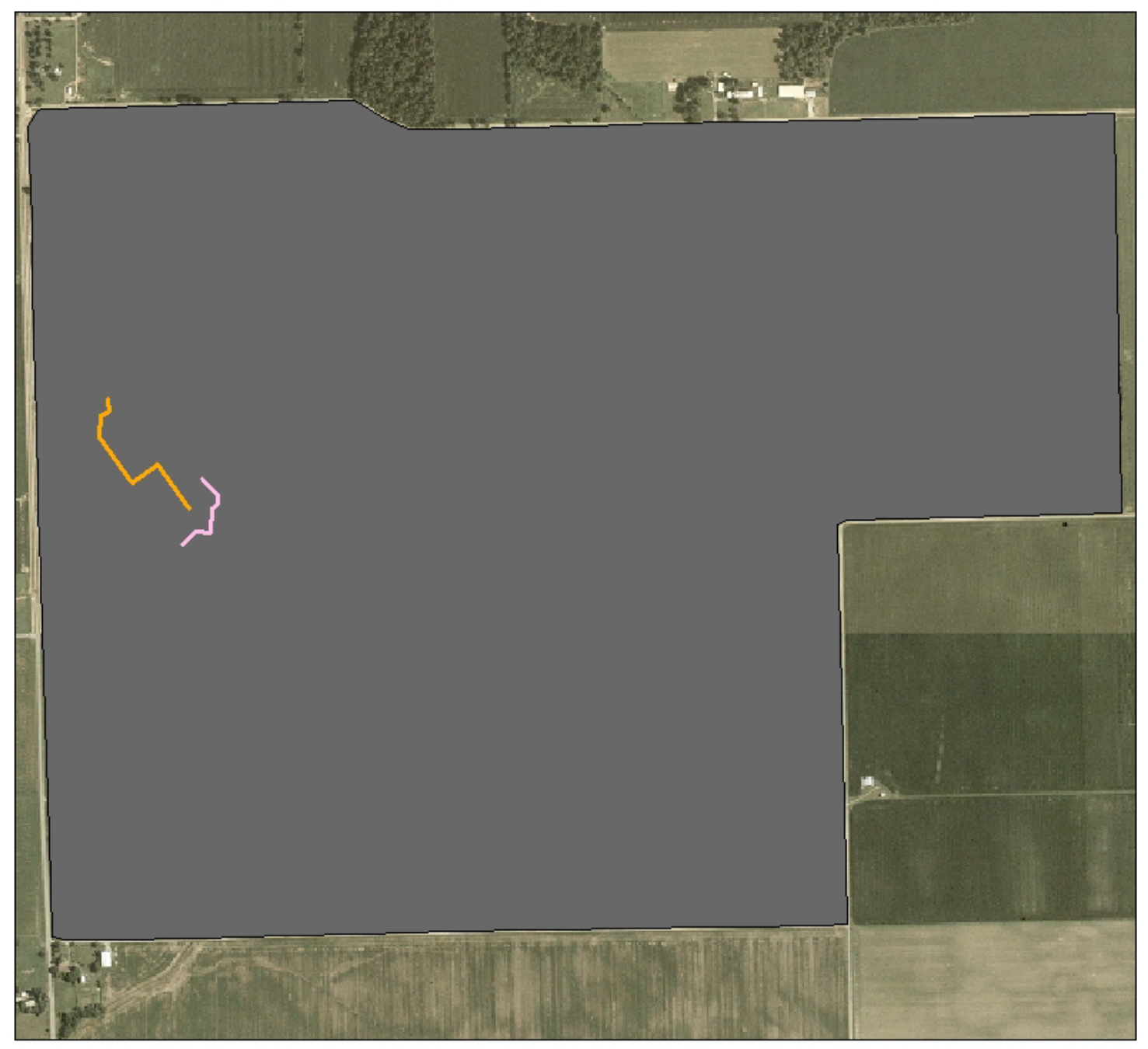

\begin{tabular}{|l|}
\hline Legend \\
$\square$ Aiport Property \\
Fence Types \\
Type G \\
Type I \\
\hline
\end{tabular}
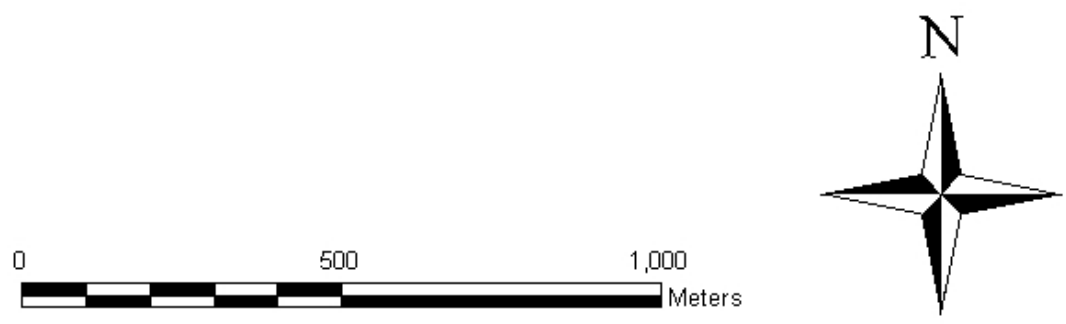

Figure 38 


\section{Airport Fencing Map: South Bend}

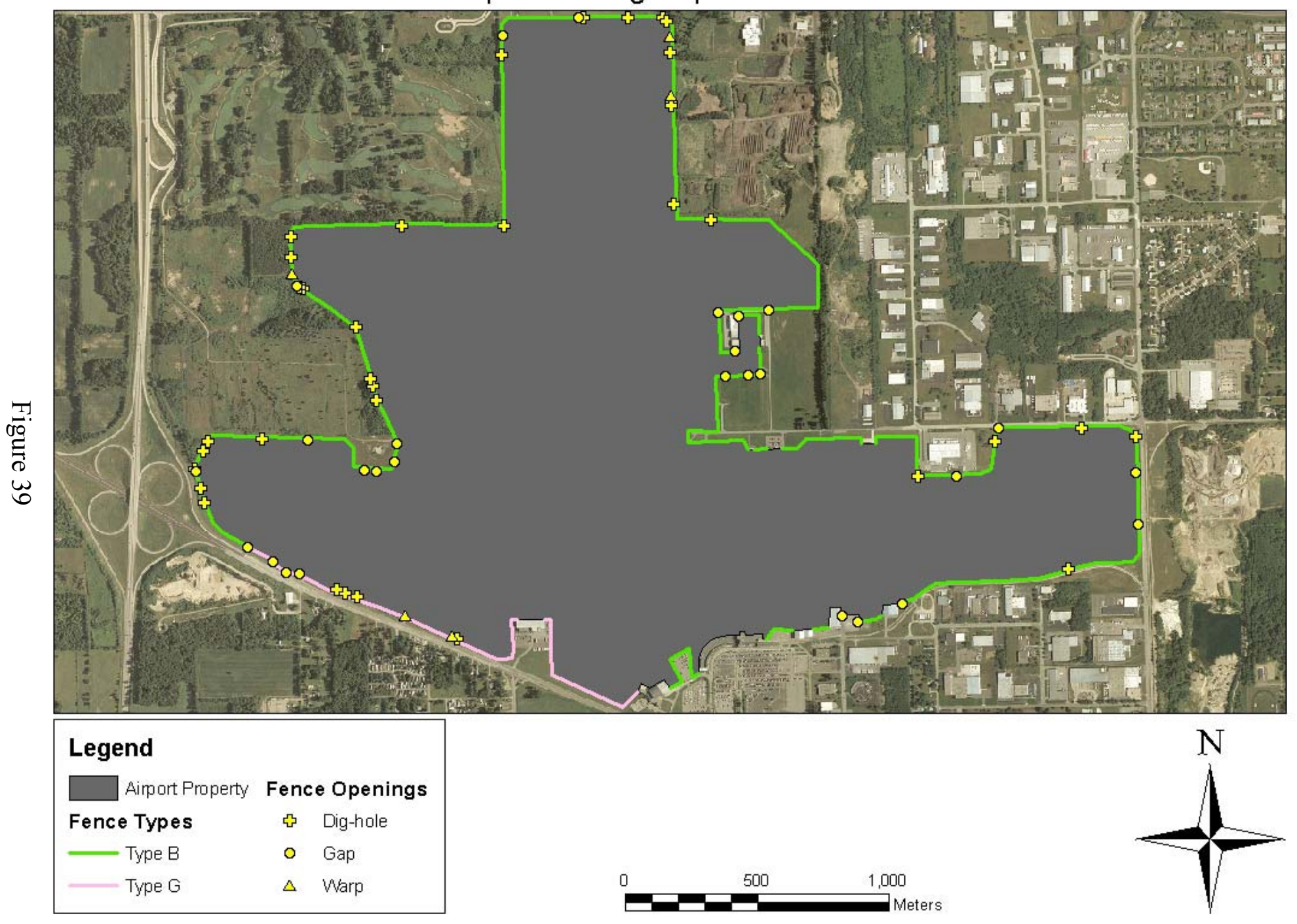




\section{Airport Fencing Map: Warsaw Municipal}

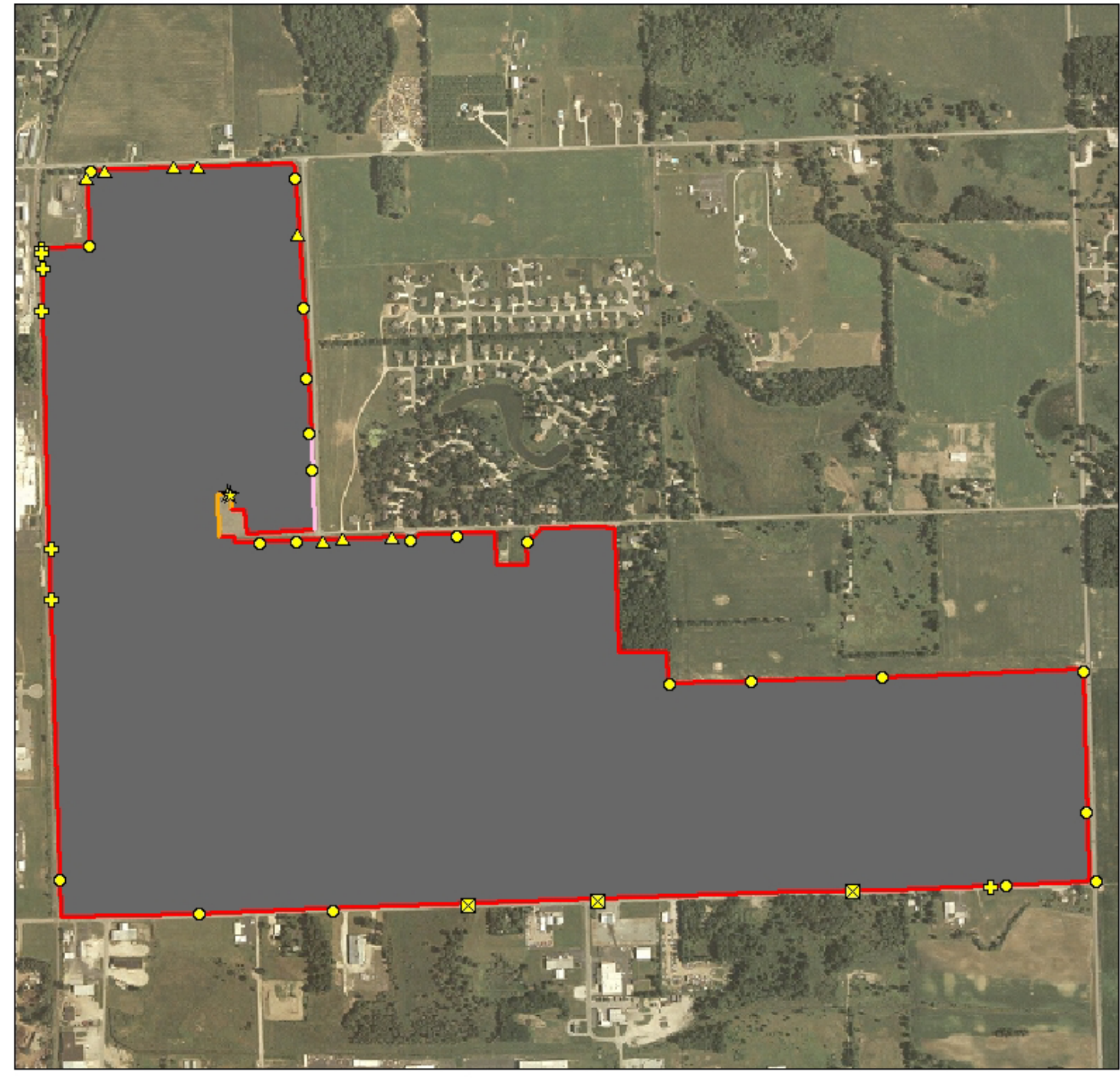

\begin{tabular}{|c|c|c|}
\hline \multicolumn{3}{|l|}{ Legend } \\
\hline Airport Property & \multicolumn{2}{|c|}{ Fence Openings } \\
\hline Fence Types & $\frac{h}{24}$ & Break \\
\hline - Type G & ほ & Dig-hole \\
\hline - Type H & 0 & Gap \\
\hline Type I & $\Delta$ & Warp \\
\hline & $\otimes$ & Other \\
\hline
\end{tabular}
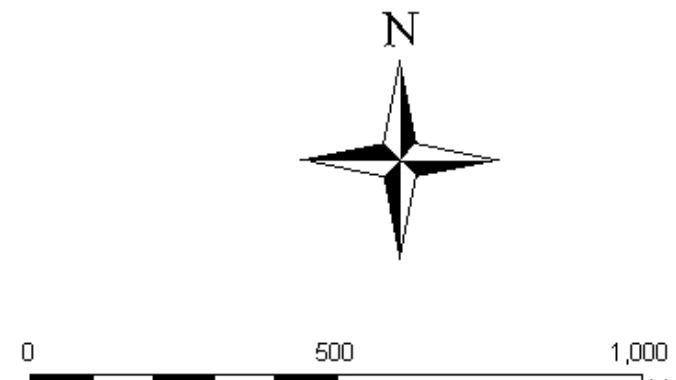

Meters

Figure 40 
Figures 41-46.

Distribution of habitat patches and relative proportion of habitat types mapped within a $10-\mathrm{km}$ radius of 3 airport properties as part of the evaluation of wildlife hazards at general aviation airports in Indiana, 2005-2006. When the 10-km radius crossed state lines, only habitats in Indiana were mapped. 


\section{0km Habitat Map: DeKalb County}

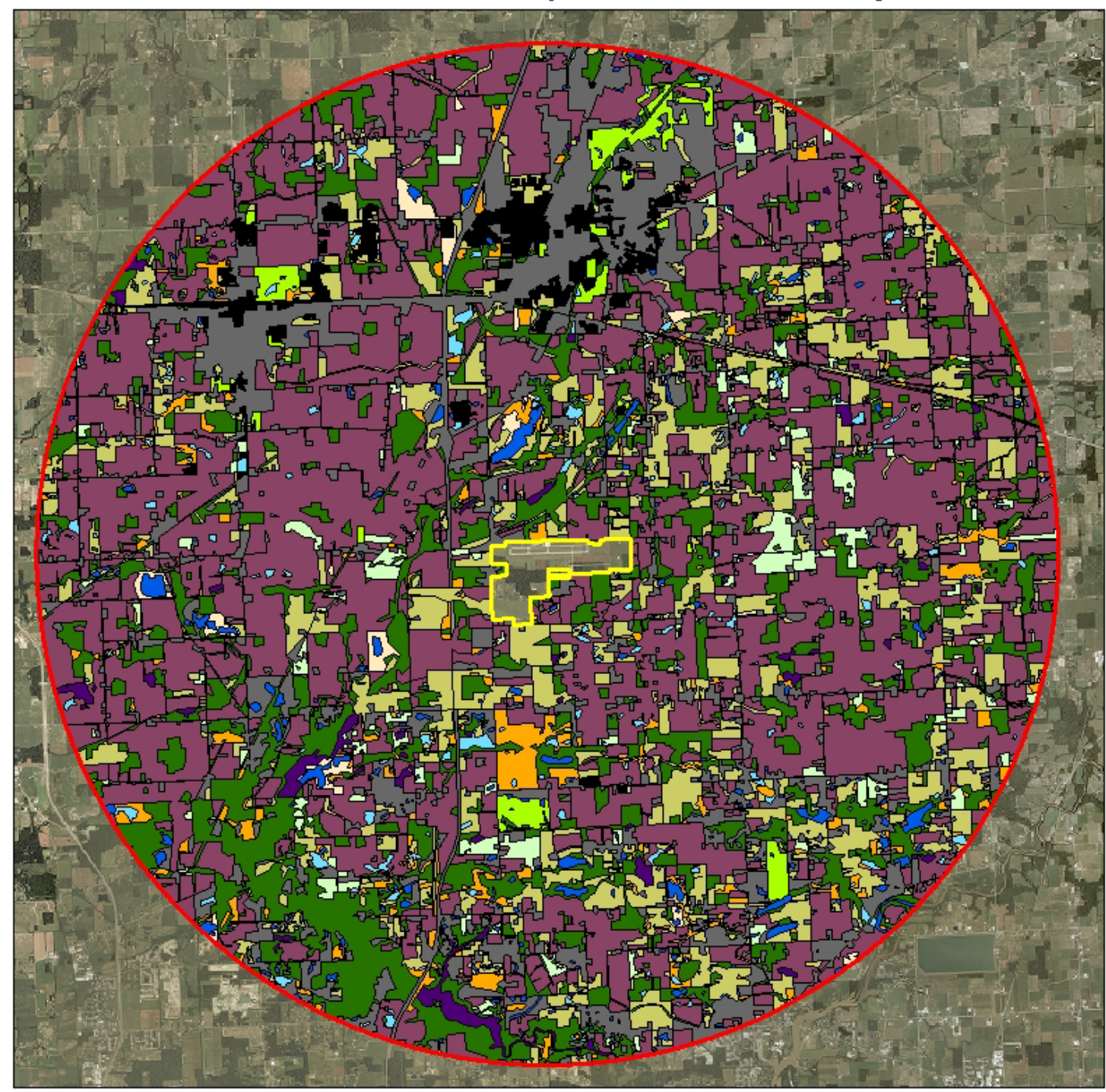

\begin{tabular}{|ll|}
\hline Legend & \\
\hline 10km Boundary & $\square$ Moderate Development \\
Airport Boundary & $\square$ Open Wetland \\
Agriculture & $\square$ Pasture \\
$\square$ Barren & $\square$ Pond/River \\
ForestMoods & $\square$ Recreational Field \\
Grassland & $\square$ Shrubland \\
Heavy Development & $\square$ Wooded Wetland \\
\hline
\end{tabular}

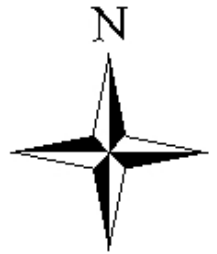

\section{Figure 41}




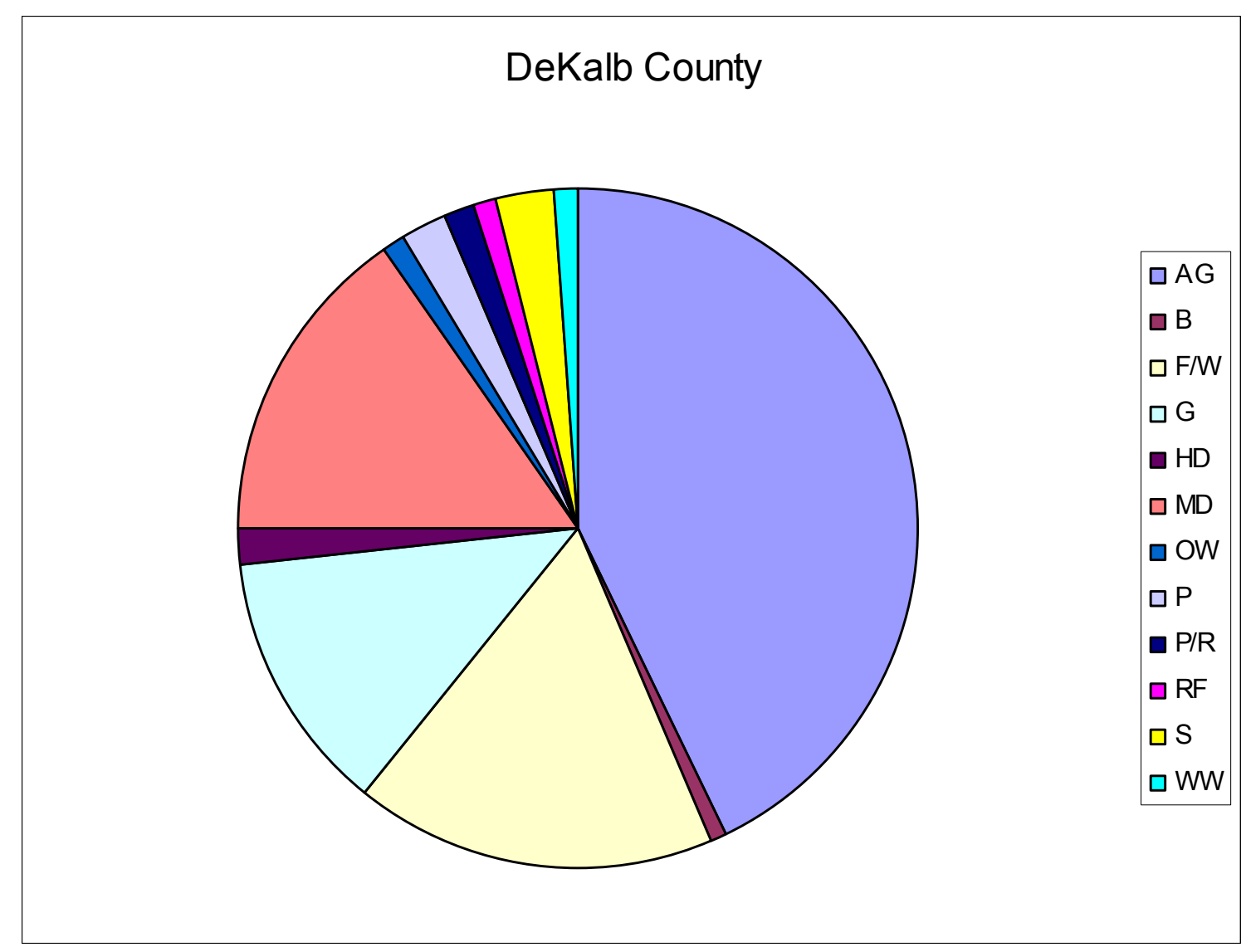

$\mathrm{AG}=$ Agriculture

$\mathrm{B}=$ Barren

$\mathrm{F} / \mathrm{W}=$ Forest $/$ Woods

$\mathrm{G}=$ Grassland

$\mathrm{HD}=$ Heavy Development

$\mathrm{MD}=$ Moderate Development

OW $=$ Open Wetland

$\mathrm{P}=$ Pasture

$\mathrm{P} / \mathrm{R}=\mathrm{Pond} /$ River

$\mathrm{RF}=$ Recreational Field

$\mathrm{S}=$ Shrubland

$\mathrm{WW}=$ Wooded Wetland

Figure 42 


\section{0km Habitat Map: South Bend Regional}
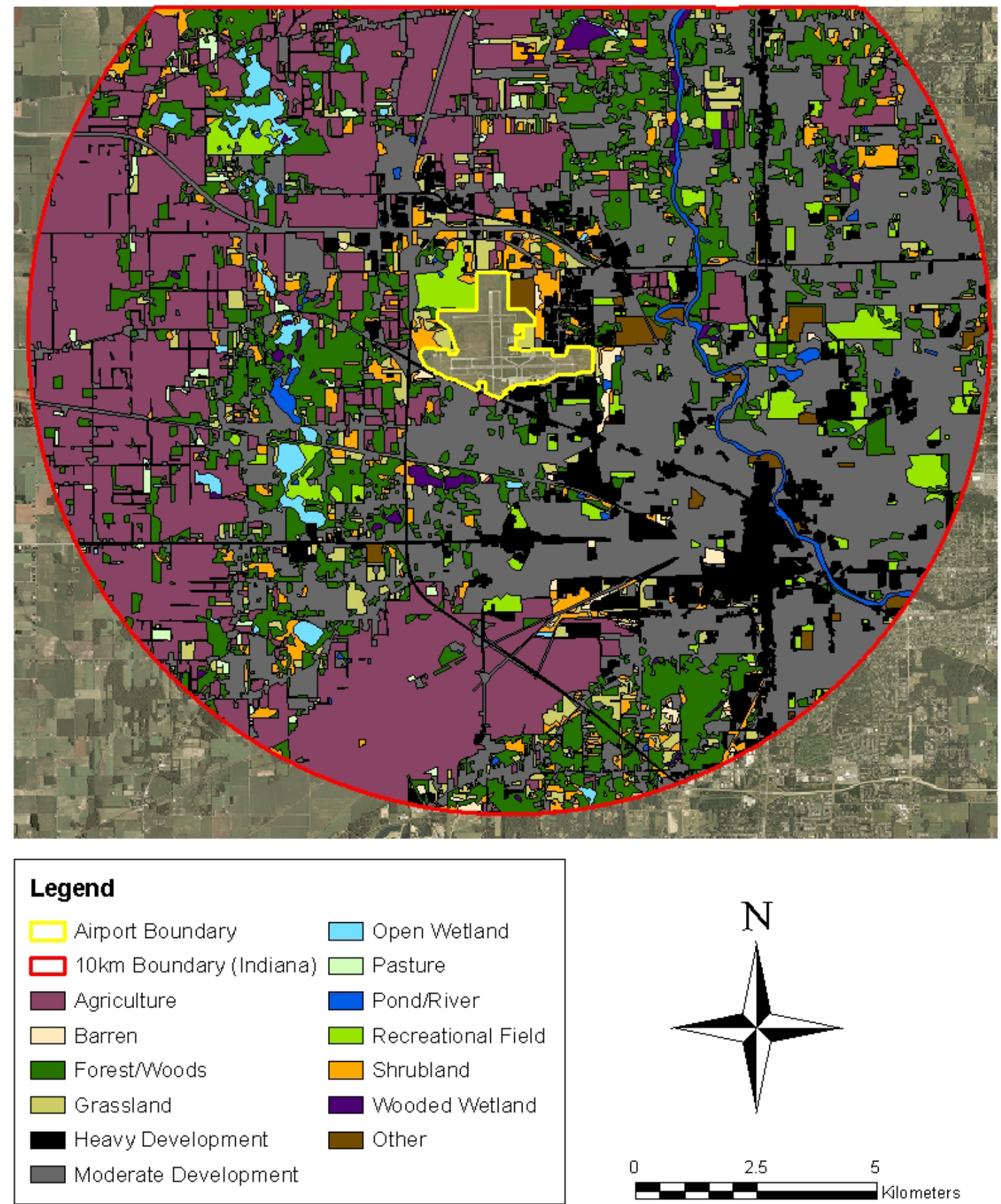

Figure 43 


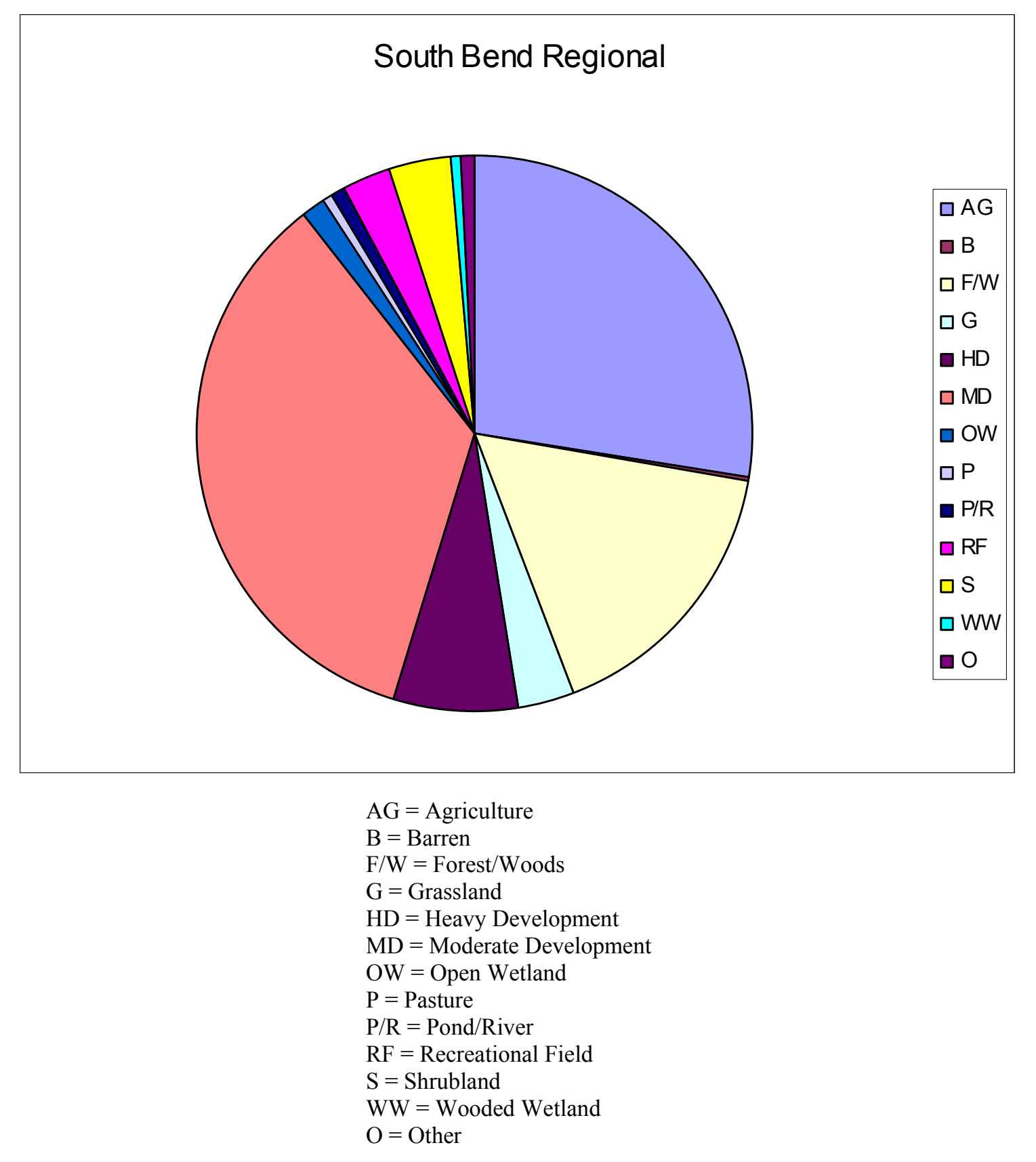

Figure 44 


\section{0km Habitat Map: Warsaw Municipal}

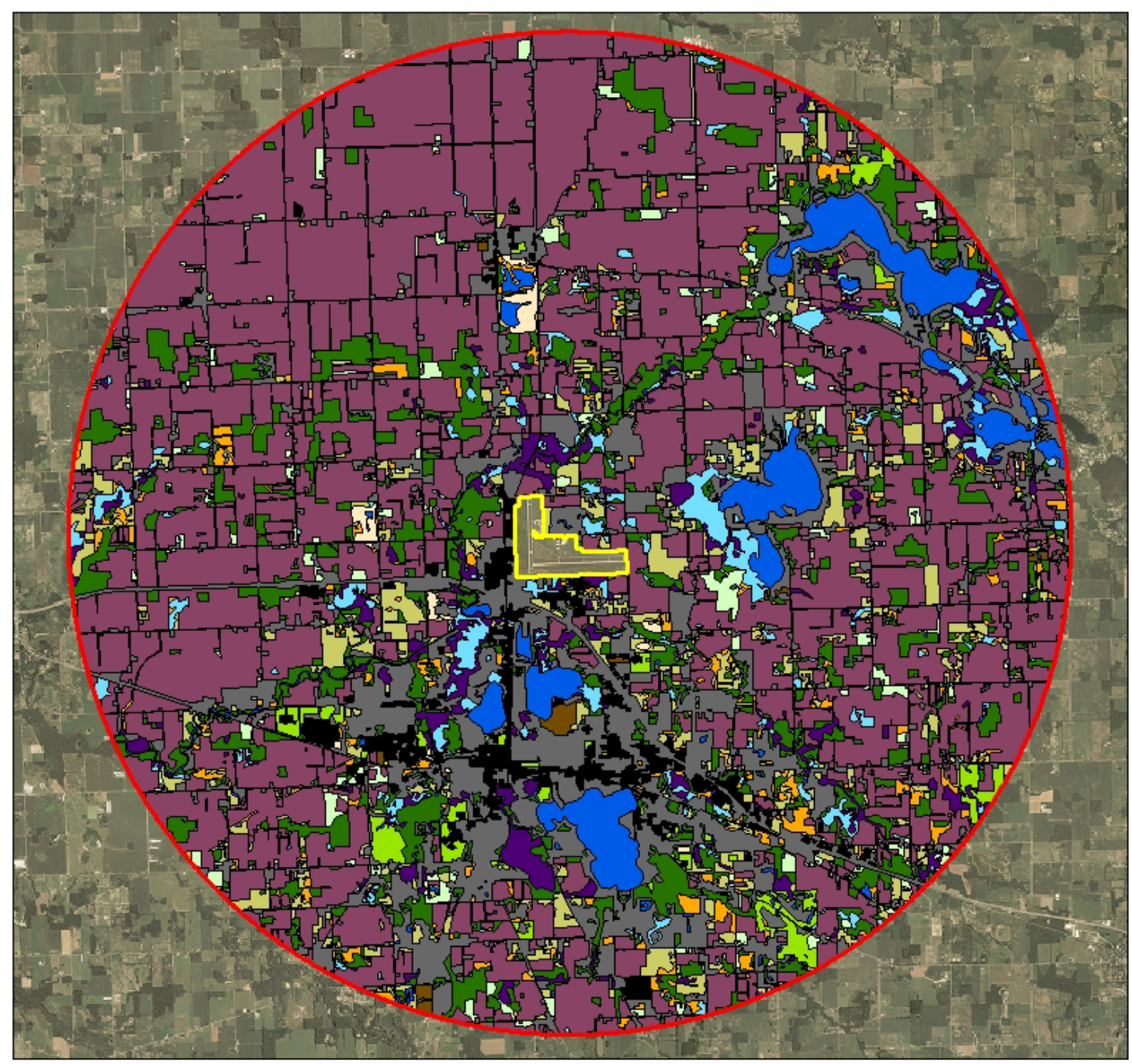

\begin{tabular}{|c|c|}
\hline \multicolumn{2}{|l|}{ Legend } \\
\hline Airport Boundary & $\square$ Open Wetland \\
\hline 10km Boundary & $\square$ Pasture \\
\hline Agriculture & Pond/River \\
\hline Barren & $\square$ Recreational Field \\
\hline Forest Woods & $\square$ Shrubland \\
\hline$\square$ Grassland & Wooded Wetland \\
\hline Heaw Development & Other \\
\hline Moderate Development & \\
\hline
\end{tabular}
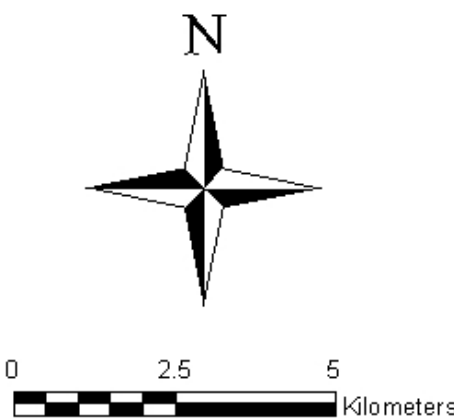

Figure 45 


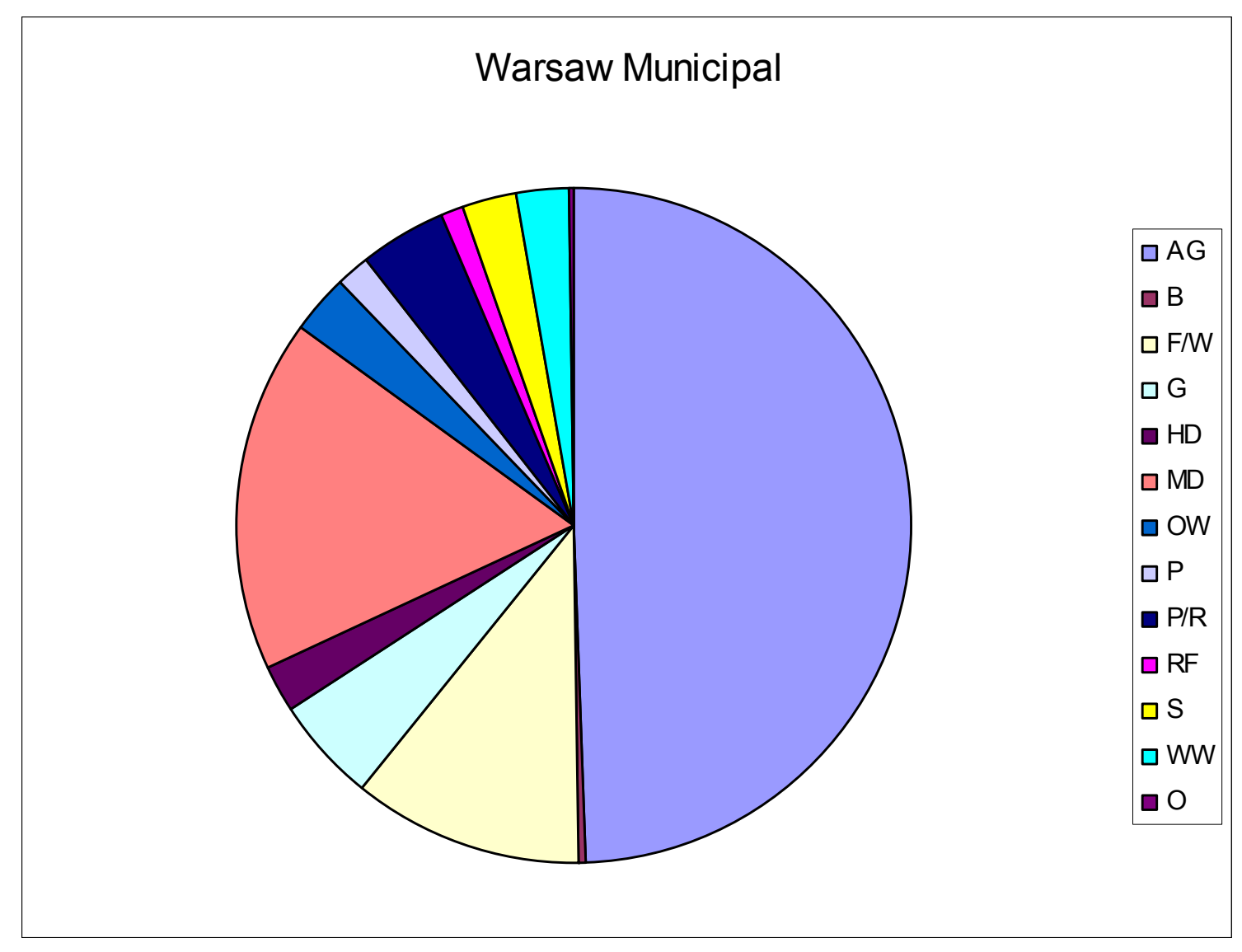

$A G=$ Agriculture

$\mathrm{B}=$ Barren

F/W $=$ Forest $/$ Woods

$\mathrm{G}=$ Grassland

$\mathrm{HD}=$ Heavy Development

$\mathrm{MD}=$ Moderate Development

OW $=$ Open Wetland

$\mathrm{P}=$ Pasture

$\mathrm{P} / \mathrm{R}=\mathrm{Pond} /$ River

$\mathrm{RF}=$ Recreational Field

$\mathrm{S}=$ Shrubland

$\mathrm{WW}=$ Wooded Wetland

$\mathrm{O}=$ Other

Figure 46 


\section{APPENDIX \\ (For each question, the number of responses for each option is given in bold and italics)}

\section{Dear Aircraft Operator,}

Purdue University is conducting an evaluation of hazards posed by wildlife at general aviation airports in Indiana. As part of our research we are contacting aircraft operators to request their participation in a short survey of their experiences at Indiana airports in general, and at 10 airports specifically: Anderson Municipal, Auburn-DeKalb County, Clark County, GreencastlePutnam County, Huntingburg, Greenwood Municipal, Purdue University, Richmond Municipal, South Bend Regional, and Warsaw Municipal. The main objectives of our survey are to gather information about 1) types of wildlife that aircraft operators most commonly observe at the airports, 2) occurrence of aircraft-operation problems caused by presence of wildlife in air operations areas, and 3) opinions of operators regarding the significance of hazards posed by various types of wildlife. Additionally, we ask questions that will help us group survey results by airport, type of aircraft, and general flight activity (e.g., peak vs. non-peak flying seasons).

We would greatly appreciate your participation in this important research project, as the information we gather may be used to develop specific management recommendations for improving hazardous conditions caused by the presence of wildlife at airports where you operate. After all, you are the operator, so your insights regarding this problem are of greatest significance!

The survey is 4 pages in length, including an optional section for respondents to provide comments, details, or clarifications for all answers provided. Please follow instructions presented throughout this document and mail your completed survey to us in the pre-addressed, stamped envelope included in this packet. We anticipate that the survey will take approximately 15 minutes to complete. Your participation is voluntary and all responses will be anonymous. If possible, please return your completed survey by January 1, 2006.

Thank you for contributing to our assessment of wildlife hazards at Indiana airports.

Sincerely,

Travis L. DeVault

Postdoctoral Research Associate

Jacob E. Kubel

Research Technician 
1. For how many years have you operated aircraft, regardless of airport/location? (please circle one)
a. $<1$ year 1
b. $1-5$ years 10
c. $6-10$ years 9
d. 11-20 years 14
e. $>20$ years 41

2. For this question, we ask for information about the 10 focal airports listed in the table. Please mark all boxes that apply for each part of the question (you may provide information for as many/few airports as you wish). (not tallied-for investigator use only to determine data biases) 


\begin{tabular}{|c|c|c|c|c|c|c|c|c|c|c|c|}
\hline & & 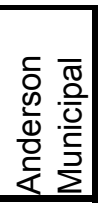 & 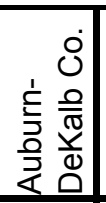 & $\begin{array}{l}0 \\
\dot{y} \\
\frac{1}{0} \\
\frac{\pi}{0}\end{array}$ & 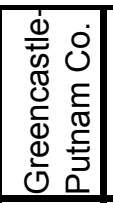 & 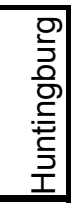 & 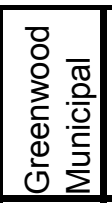 & 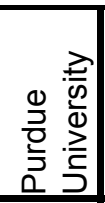 & 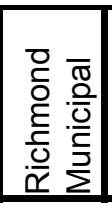 & 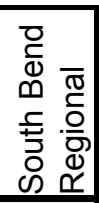 & 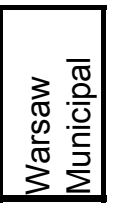 \\
\hline \multirow{4}{*}{$\begin{array}{l}\text { For how many years have you } \\
\text { operated aircraft at these } \\
\text { airports? }\end{array}$} & $<1$ year & & & & & & & & & & \\
\hline & $1-5$ years & & & & & & & & & & \\
\hline & $6-10$ years & & & & & & & & & & \\
\hline & $>10$ years & & & & & & & & & & \\
\hline \multirow{7}{*}{$\begin{array}{l}\text { What types of aircraft do you } \\
\text { operate at these airports } \\
\text { (please check all that apply)? }\end{array}$} & Piston Single & & & & & & & & & & \\
\hline & \begin{tabular}{|l|} 
Piston Multi \\
\end{tabular} & & & & & & & & & & \\
\hline & Turbine Single & & & & & & & & & & \\
\hline & Turbine Multi & & & & & & & & & & \\
\hline & Jet & & & & & & & & & & \\
\hline & Helicopter & & & & & & & & & & \\
\hline & Other & & & & & & & & & & \\
\hline \multirow{4}{*}{$\begin{array}{l}\text { Based on your recent flight } \\
\text { activity, what do you consider } \\
\text { to be your peak flying season(s) } \\
\text { at these airports? }\end{array}$} & Spring (Apr-Jun) & & & & & & & & & & \\
\hline & Summer (Jul-Sep) & & & & & & & & & & \\
\hline & Fall (Oct-Dec) & & & & & & & & & & \\
\hline & Winter (Jan-Mar) & & & & & & & & & & \\
\hline \multirow{5}{*}{$\begin{array}{l}\text { On average, how many days } \\
\text { per week did you operate } \\
\text { aircraft at these airports during } \\
\text { your peak flying season(s) this } \\
\text { past year? }\end{array}$} & $<1$ day & & & & & & & & & & \\
\hline & 1 day & & & & & & & & & & \\
\hline & 2-3 days & & & & & & & & & & \\
\hline & 4-5 days & & & & & & & & & & \\
\hline & $>5$ days & & & & & & & & & & \\
\hline \multirow{5}{*}{$\begin{array}{l}\text { On average, approximately how } \\
\text { many days per month did you } \\
\text { operate aircraft at these airports } \\
\text { during your non-peak flying } \\
\text { season(s) this past year? }\end{array}$} & $<1$ day & & & & & & & & & & \\
\hline & 1 day & & & & & & & & & & \\
\hline & 2-7 days & & & & & & & & & & \\
\hline & 8-14 days & & & & & & & & & & \\
\hline & $>14$ days & & & & & & & & & & \\
\hline \multirow{5}{*}{$\begin{array}{l}\text { On a typical day that you operate } \\
\text { aircraft during your peak flying } \\
\text { season(s), how many times do } \\
\text { you land and/or take off at these } \\
\text { airports? }\end{array}$} & 1 time each day & & & & & & & & & & \\
\hline & 2 times each day & & & & & & & & & & \\
\hline & 3-4 times each day & & & & & & & & & & \\
\hline & $5-10$ times each day & & & & & & & & & & \\
\hline & $>10$ times each day & & & & & & & & & & \\
\hline \multirow{5}{*}{$\begin{array}{l}\text { On a typical day that you operate } \\
\text { aircraft during your non-peak } \\
\text { flying season(s), how many times } \\
\text { do you land and/or take off at } \\
\text { these airports? }\end{array}$} & 1 time each day & & & & & & & & & & \\
\hline & 2 times each day & & & & & & & & & & \\
\hline & 3-4 times each day & & & & & & & & & & \\
\hline & $5-10$ times each day & & & & & & & & & & \\
\hline & $>10$ times each day & & & & & & & & & & \\
\hline
\end{tabular}

3. Which of the 10 focal airports listed in the previous table do you use most often (which is your primary airport)? (please circle one)

1) Anderson Municipal 3

2) Auburn-DeKalb County 13

3) Clark County 14

4) Greencastle-Putnam County 9

5) Huntingburg 7 

6) Greenwood Municipal 2
7) Purdue University 18
8) Richmond Municipal 5
9) South Bend Regional 4
10) Warsaw Municipal 9

4. For this question, we ask for information about 11 groups of wildlife that occasionally cause problems at airports. We ask that you answer the question only with regard to your primary airport (as answered in Question 3 above). Please mark all boxes that apply.

\begin{tabular}{|c|c|c|c|c|c|c|c|c|c|c|c|c|}
\hline & & & & & & & & & & & & \\
\hline \multirow{6}{*}{$\begin{array}{l}\text { During your peak flying season(s), } \\
\text { approximately how often do you } \\
\text { observe the following types of } \\
\text { wildlife in air operations areas } \\
\text { of your primary airport? }\end{array}$} & $>75 \%$ of flights & 12 & 1 & 5 & 0 & 0 & 3 & 10 & 15 & $I$ & 1 & 0 \\
\hline & $51-75 \%$ of flights & 11 & 0 & 8 & 0 & 0 & 4 & 4 & 6 & 0 & 2 & \\
\hline & $25-50 \%$ of flights & 12 & 2 & 15 & 7 & 5 & 7 & 22 & 15 & 6 & & \\
\hline & $<25 \%$ of flights & 31 & 12 & 40 & 29 & 13 & 23 & 26 & 27 & 43 & 31 & 23 \\
\hline & Never & 9 & 36 & 9 & 23 & 34 & 17 & 6 & 3 & 18 & 25 & 31 \\
\hline & Do not know & 1 & 8 & 2 & 5 & 9 & 10 & 5 & 5 & 3 & 8 & \\
\hline \multirow{6}{*}{$\begin{array}{l}\text { During your non-peak flying } \\
\text { seasons, approximately how often } \\
\text { do you observe the following types } \\
\text { of wildlife in air operations areas } \\
\text { of your primary airport? }\end{array}$} & $>75 \%$ of flights & 9 & 0 & 3 & 0 & 0 & 2 & 8 & 14 & 1 & 1 & 0 \\
\hline & $51-75 \%$ of flights & 12 & 1 & 10 & 0 & 0 & 5 & 3 & 3 & 1 & 4 & 1 \\
\hline & $25-50 \%$ of flights & 6 & 2 & 9 & 6 & 3 & 5 & 22 & 9 & 6 & 4 & 1 \\
\hline & $<25 \%$ of flights & 34 & 11 & 41 & 29 & 17 & 24 & 25 & 31 & 39 & 28 & 22 \\
\hline & Never & 10 & 35 & 9 & 21 & 30 & 18 & 7 & 5 & 17 & 24 & 28 \\
\hline & Do not know & 2 & 8 & 2 & 5 & 9 & 8 & 4 & 5 & 6 & 8 & 9 \\
\hline \multirow{5}{*}{$\begin{array}{l}\text { In your opinion, how hazardous are } \\
\text { these types of wildlife in the air } \\
\text { operations areas of your primary } \\
\text { airport with respect to human } \\
\text { safety? }\end{array}$} & Very hazardous & 15 & 5 & 21 & 5 & 3 & 3 & 8 & 3 & 23 & 11 & \\
\hline & Hazardous & 9 & 6 & 15 & 14 & 8 & 9 & 19 & 10 & 16 & 12 & 10 \\
\hline & Somewhat hazardous & 30 & 12 & 34 & 24 & 19 & 25 & 32 & 26 & 18 & 21 & 16 \\
\hline & Not hazardous & 18 & 27 & 9 & 20 & 24 & 27 & 13 & 33 & 14 & 23 & 27 \\
\hline & No opinion & 3 & 13 & 1 & 3 & 9 & 2 & 2 & 2 & 3 & 4 & 9 \\
\hline \multirow{5}{*}{$\begin{array}{l}\text { In your opinion, how hazardous are } \\
\text { these types of wildlife in the air } \\
\text { operations areas of your primary } \\
\text { airport with respect to economic } \\
\text { costs of a wildlife collision? }\end{array}$} & Very hazardous & 17 & 5 & 19 & 5 & 3 & 3 & 13 & 3 & 24 & 15 & \\
\hline & Hazardous & 21 & 9 & 19 & 16 & 8 & 10 & 15 & 10 & 15 & 16 & 12 \\
\hline & Somewhat hazardous & 19 & 13 & 28 & 19 & 21 & 24 & 25 & 31 & 18 & 17 & 18 \\
\hline & Not hazardous & 12 & 23 & 6 & 17 & 18 & 22 & 13 & 22 & 8 & 14 & 21 \\
\hline & No opinion & 7 & 13 & 7 & 9 & 13 & 7 & 7 & 8 & 8 & 8 & 11 \\
\hline
\end{tabular}


5. During the past year at your primary airport, how many times have you had to alter flight, landing, or take-off plans because of wildlife occurring within the air operations areas? (please circle one)
a. 0 times 26
b. 1-2 times 33
c. 3-5 times $\mathbf{1 8}$
d. 6-10 times 5
e. $>10$ times 2

6. During the past year at your primary airport, how many times have you been involved in a collision with wildlife while operating aircraft within the air operations areas? (please circle one)
a. 0 times 63
b. 1-2 times 20
c. 3-5 times 1
d. 6-10 times 0
e. $>10$ times 0

7. In terms of wildlife presence and abundance that you have observed, to what degree do you believe improvement of current conditions or management strategies at your primary airport is needed to ensure that wildlife are not a hazard in air operations areas? (please circle one)
a. Needs much improvement $\mathbf{1 8}$
b. Needs a little improvement $\mathbf{3 1}$
c. Does not need improvement 28
d. No opinion 6

8. Over the entire course of your experience as a pilot, how many times have you been involved in a collision with wildlife while operating aircraft, regardless of airport/location? (please circle one)
a. 0 times 19
b. 1-2 times 31
c. 3-5 times 19
d. 6-10 times 9
e. $>10$ times 6

9. With which of the following types of wildlife have you been involved in an aircraft-wildlife collision, regardless of airport/location? Please circle all that apply. If you have information that is more specific than implied by one of the choices below, please explain via choice "n. Other than above."
a. Not applicable 17
h. Starling, blackbird, or grackle 36 

b. Large soaring bird (eagle, hawk, or vulture) $\mathbf{1 2}$
i. Dove/Pigeon 8
c. Heron or Crane 1
d. Goose 17
j. Other bird
11
e. Duck 9
k. Deer 6
f. Gull 9
1. Coyote 3
m. Other mammal
g. Crow/Raven 10
n. Other than above
2

10. Have your experiences with wildlife ever caused you to permanently cease operating aircraft at a particular airport? If answering "yes," you may specify the airport and state if you wish.
a. Yes
3
b. No 81

11. With respect to potential for aircraft-wildlife collisions, how hazardous are wildlife populations (in general) at all airports in Indiana? (please circle one)
a. Very hazardous (a significant problem requiring immediate action) 8
b. Hazardous (a problem, but immediate action is not necessary) 28
c. Somewhat hazardous (a problem only under rare circumstances, and action probably is not necessary) 36
d. Not hazardous (not a problem, no action necessary). 9

12. Wildlife problems often are addressed in different ways. In general, to what degree do you support the following actions that potentially may prevent or reduce frequency of aircraftwildlife collisions? (please circle one choice for each action)

A. Construction of exclosures (fencing) habitat
a. Strongly support 35
b. Support 21
c. Neither support nor oppose 15
d. Oppose 8
e. Strongly oppose 2

B. Use of wildlife deterrents (e.g., loud lethal sounds, flashing lights, owl decoys)
a. Strongly support $\mathbf{2 3}$
b. Support 32
c. Neither support nor oppose 19
d. Oppose 7
e. Strongly oppose 1

C. Modification/elimination of wildlife
a. Strongly support 16
b. Support 19
c. Neither support nor oppose $\mathbf{1 6}$
d. Oppose 22
e. Strongly oppose 9

D. Direct removal of wildlife (involves means)
a. Strongly support 15
b. Support 16
c. Neither support nor oppose 14
d. Oppose 26
e. Strongly oppose 11

13. Please feel free to add comments or clarifications to answers you provided for any of the previous questions:

Thank you for your participation! 